NEUROANTIGEN-SPECIFIC CD8+ REGULATORY T-CELL FUNCTION IS DEFICIENT DURING ACUTE EXACERBATION OF MULTIPLE

SCLEROSIS

APPROVED BY SUPERVISORY COMMITTEE

Nitin Karandikar MD PhD

Nancy Street PhD

Dwain Thiele MD

James Forman PhD DMD

Benjamin Greenberg MD MHSc 


\section{DEDICATION}

I would like to dedicate this work in honor of my wife, daughter, and parents. 


\title{
NEUROANTIGEN-SPECIFIC CD8+ REGULATORY T-CELL FUNCTION IS DEFICIENT DURING ACUTE EXACERBATION OF MULTIPLE SCLEROSIS
}

\author{
by \\ ETHAN JAMES BAUGHMAN
}

\section{DISSERTATION /THESIS}

Presented to the Faculty of the Graduate School of Biomedical Sciences

The University of Texas Southwestern Medical Center at Dallas

In Partial Fulfillment of the Requirements

For the Degree of

DOCTOR OF PHILOSOPHY

The University of Texas Southwestern Medical Center at Dallas

Dallas, Texas

June, 2011 


\section{Copyright}

by

\section{ETHAN JAMES BAUGHMAN, 2011}

All Rights Reserved 


\section{ACKNOWLEDGEMENTS}

Foremost, I thank my principal investigator Dr. Nitin Karandikar. During the six years since I was first introduced to Nitin as an undergraduate, he has truly shown me how to live as a physician scientist and a good person. As a mentor, Nitin has always been enthusiastic yet grounded, extremely knowledgeable though approachable. Nitin taught me to think critically, make hypotheses, ask important questions, and design proper experiments. Despite his very busy schedule, Nitin listened to my concerns, mentored and advised me about science and life generally. I have always felt respected and acknowledged at home and in the field because of Nitin.

I would also like to personally thank the members of my committee, Drs. Nancy Street, Dwain Thiele, James Forman, Benjamin Greenberg, for their mentoring and guiding of my career. I could not have gained acceptance at UT Southwestern from undergraduate college without the guidance and support of Drs. Ruth Welti and Larry Williams. I would also like to thank Drs. Elliot Frohman, Todd Eagar, Olaf Stuve, Larry Anderson, Mihail Firan, David Farrar, Mark Siegelman, Pila Estess, Vinodh Pillai, Nathan York, Jason Mendoza, Sterling Ortega, Chris Ayers, and Venkatesh Kashi for their scientific and intellectual contributions to my career. We are all indebted to all the patients who participated in these studies. For help with patient recruitment, I thank Michael Racke, Thomas Abraham, Stephanie Taylor, Megan Quigg, and Parul Chaudhary. For running the labs and helping me with my experiments, I thank Rich Grother, Brian Biegler, Andy Benagh, Jana Windsor, Maycie Garibay, Tommy Lee, Hunter Baldwin, Liz Gunter, Jamie Houser, Carlos Aviles, Matthew Cummings, Andrew Tyler, and Imran Mohiuddin. I would like to thank all my lab mates past and present who all made it such a fun, productive lab. I am thankful for so many life-long friends that I have made in graduate school and the medical scientist training program (MSTP) at lunch breaks and happy hours. In administration of the MSTP and immunology graduate program, I thank Andrew Zinn, Mike Brown, Robin Downing, Stephanie Robertson, Harmony Hilton, and Karen Kazemzadeh.

In addition to all my other friends and family, I especially thank my wife, Dr. Christen Buseman, and daughter, Evelyn Rose, for their love. With my beautiful women, life takes on a shine. For insightful scientific discussions as well as moral support, I would like to thank Drs. Daniel Parente and Jennifer Buseman. I would like to thank my parents-in-law, Randy Buseman, and Cheryl Buseman, for never-ending love, encouragement, and support. I thank my parents, Gary and Joyce, and brother, Duane, for their sacrifices, love, support, and encouragement for everything I wanted to pursue in life. My father, Dr. Gary Baughman, DVM, was my first attending, principal investigator, and mentor, and has influenced my career in countless ways. 


\section{NEUROANTIGEN-SPECIFIC CD8+ REGULATORY T-CELL FUNCTION IS DEFICIENT DURING ACUTE EXACERBATION OF MULTIPLE SCLEROSIS}

Publication No.

ETHAN JAMES BAUGHMAN, Ph.D.

The University of Texas Southwestern Medical Center at Dallas, 2011

NITIN J. KARANDIKAR, M.D. Ph.D.

Multiple sclerosis (MS) is an inflammatory, demyelinating disease of the central nervous system (CNS). MS is thought to be T-cell-mediated, with prior research predominantly focusing on CD4+ T-cells. There is a high prevalence of 
CNS-specific CD8+ T-cell responses in MS patients and healthy subjects. However, the role of neuroantigen-specific CD8+ T-cells in MS is poorly understood, with the prevalent notion that these may represent pathogenic T-cells. We show here that healthy subjects and MS patients demonstrate similar magnitudes of $\mathrm{CD} 8+$ and $\mathrm{CD} 4+\mathrm{T}$-cell responses to various antigenic stimuli. Interestingly, CD8+ T-cells specific for CNS autoantigens, but not those specific for control foreign antigens, exhibit immune regulatory ability, suppressing proliferation of $\mathrm{CD} 4+\mathrm{CD} 25-\mathrm{T}$-cells when stimulated by their cognate antigen. While CD8+ T-cell-mediated immune suppression is similar between healthy subjects and clinically quiescent treatment-naïve MS patients, it is significantly deficient during acute exacerbation of MS. Of note, the recovery of neuroantigenspecific CD8+ T-cell suppression correlates with disease recovery post-relapse. In healthy adult subjects, we observed that the CD62L- subset of CD8+ T cells harbored increased CNS- and Copaxone-specific suppressive ability, when compared to the CD62L+ subset and bulk CD8+ $\mathrm{T}$ cells, and that the CD28+ subset of CD8+ $\mathrm{T}$ cells harbored increased global suppressive ability, when compared to the CD28- subset. In contrast, we observed CD8+ $\mathrm{T}$ cells from neonates harbored increased global suppressive ability in the CD28- subset. The mechanism of neuroantigen-specific suppression by CD8+ T cells was dependent upon HLA class I, IFN $\gamma$, with possible partial involvement by NKG2D, PD-1, and IL10. These studies reveal a novel immune suppressor function for neuroantigenspecific CD8+ T-cells that is clinically relevant in the maintenance of peripheral tolerance and the intrinsic regulation of MS immune pathology 
TABLE OF CONTENTS

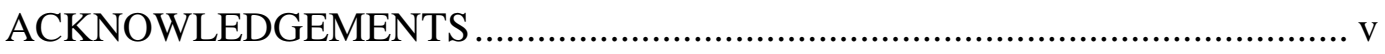

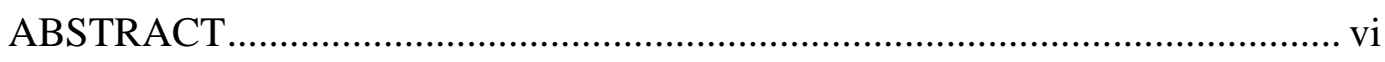

TABLE OF CONTENTS ....................................................................... viii

PRIOR PUBLICATIONS AND ABSTRACTS ........................................... xii

LIST OF FIGURES .........................................................................

LIST OF TABLES ................................................................................. $\mathrm{xvi}$

LIST OF ABBREVIATIONS ................................................................... xvii

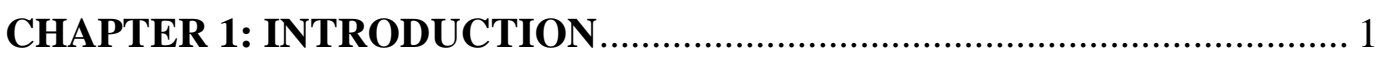

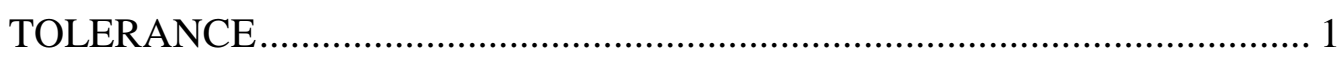

CENTRAL TOLERANCE ............................................................. 1

PERIPHERAL TOLERANCE........................................................... 3

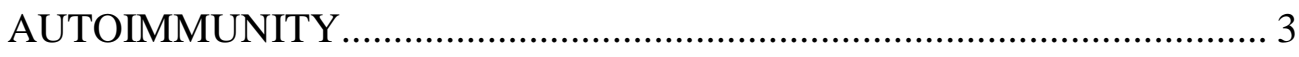

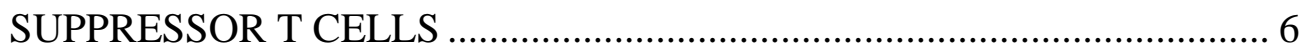

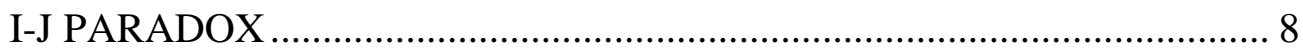

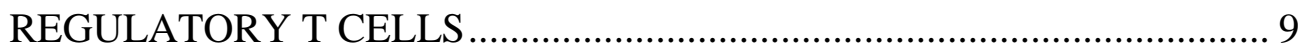

ANTIGEN-SPECIFIC CD8+ TREGS ................................................. 12

GLOBALLY SUPPRESSIVE, NON-SPECIFIC CD8+ TREGS................ 13

TRANSCRIPTIONA.L REGULATION OF TREG ............................... 17

SUPPRESSIVE PROPERTY OF ACTIVATED FOXP3+ T CELLS ......... 18

IMMUNE-MEDIATED DYMELINATION ............................................ 20

MULTIPLE SCLEROSIS ............................................................... 22

EXPERIMENTAL AUTOIMMUNE ENCEPHALOMYELITIS ............... 32

DEFICIENT CENTRAL TOLERANCE IN MS ..................................... 49

DEFICIENT PERIPHERAL TOLERANCE IN MS ............................... 50

CD8+ T CELLS IN HEALTH AND DISEASE......................................... 52

AUTOREACTIVE REGULATORY CD8+ T CELLS ............................. 54

AUTOREACTIVE CD8+ T CELLS AND MS ....................................... 56

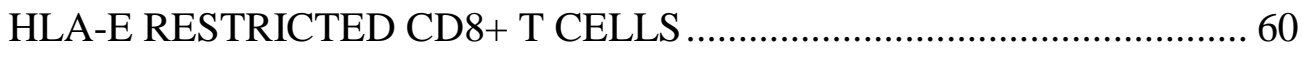

POTENIAL FOR CELL BASED THERAPIES IN AUTOIMMUNITY AND

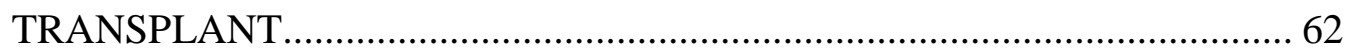

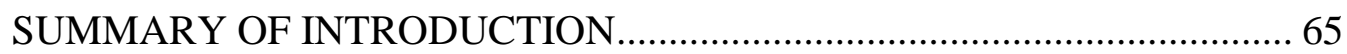




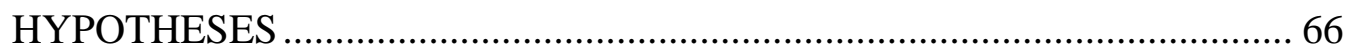

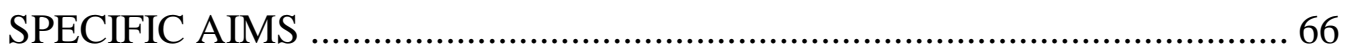

CHAPTER 2: METHODS AND MATERIALS ………………………......... 68

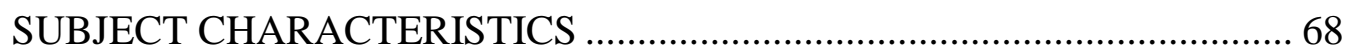

CELL PREPARATION AND BEAD SORTING ………………………....... 70

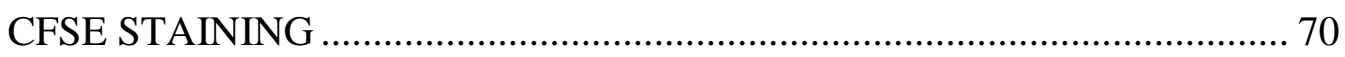

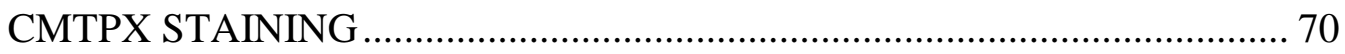

FLOW CYTOMETRY-BASED SUPPRESSION ASSAY CULTURES ......... 71

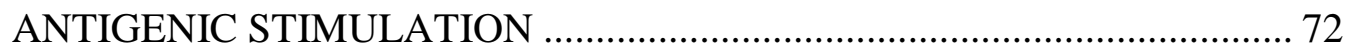

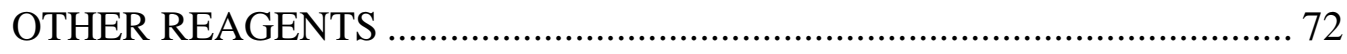

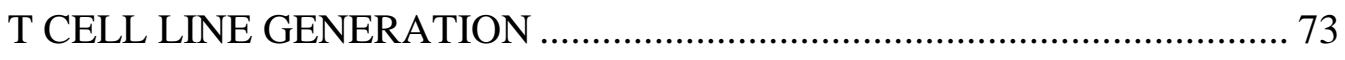

FLOW CYTOMETRIC ANTIBODY STAINING ……………………….... 75

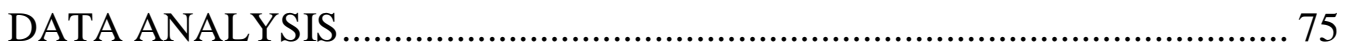

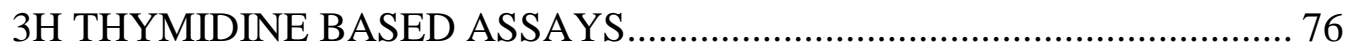

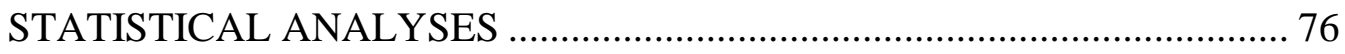

STANDARDIZATION OF MIXED LYMPHOCYTE REACTION (MLR) .. 77

MLR-BASED SUPPRESSION ASSAY ………………………………..... 77

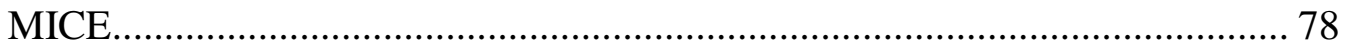

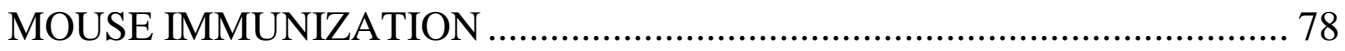

MURINE FLOW-BASED SUPPRESSION ASSAY ........................................ 78

SHRNA KNOCKDOWN OF ACTIVATED AND NATURAL FOXP3 …..... 79

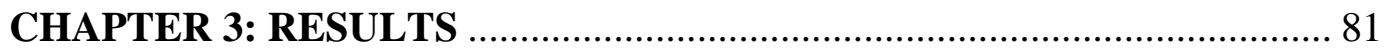

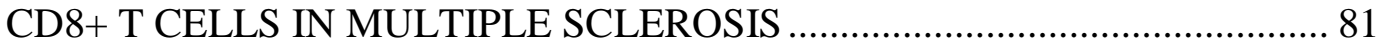

MULTIPLE SCLEROSIS PATIENTS AND HEALTHY CONTROL

SUBJECTS SHARE SIMILAR T CELL RESPONSES ................................... 81

CD8+ T-CELLS SPECIFIC FOR CNS AUTOANTIGENS, BUT NOT THOSE SPECIFIC FOR CONTROL FOREIGN ANTIGENS, SUPPRESS CD4+ T-

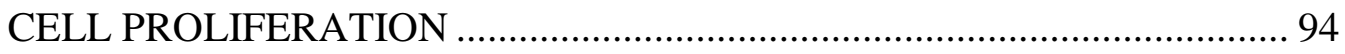

CNS-SPECIFIC CD8+ T-CELLS REQUIRE STIMULATION WITH COGNATE ANTIGEN FOR SUPPRESSIVE ACTIVITY

CNS-SPECIFIC SUPPRESSIVE ABILITY IS SIGNIFICANTLY

DIMINISHED DURING ACUTE EXACERBATION OF MS AND

RECOVERS DURING REMISSION..

NEUROANTIGEN-SPECIFIC CD8+ T CELL SUPPRESSIVE ABILITY

CORRELATES WITH DAYS SINCE LAST RELAPSE 
NEUROANTIGEN-SPECIFIC CD8+ T CELL SUPPRESSIVE ABILITY CORRELATES WITH DAYS SINCE LAST RELAPSE AND RECOVERY

NEUROANTIGEN-SPECIFIC CD8 SUPPRESSION REQUIRES HLA CLASS I AND INTERFERON GAMMA

ANTIGEN-SPECIFIC SUPPRESSION IS HARBORED IN THE CD8+CD62L- SUBSET

GLOBAL SUPPRESSIVE ABILITY IN ADULTS IS HARBORED IN THE CD8+CD28+ SUBSET 128

CHAPTER 4: DISCUSSION 130

DEFICIENT CNS-SPECIFIC CD8 TREG IN MS ACUTE

EXACERBATIONS 130

IMMUNOLOGIC EPIDEMIOLOGY 130

LIMITATIONS AND MINIMIZING ERROR 133

AUTOREGULATORY T CELLS. 134

IMMUNE THERAPY USING CD8+ T CELLS. 135

FUNDAMENTAL DIFFERENCES BETWEEN AUTOREACTIVE AND FOREIGN SPECIFIC T CELLS 136

PATHOGENIC ROLE FOR INFILTRATING CD8+ T CELLS IN MS ...... 140 MODEL

FUTURE DIRECTIONS

PROPOSED MODEL OF CNS-SPECIFIC REGULATORY T CELLS DYSFUNCTION DURING ACUTE EXACERBATION OF MS

NOVEL FLOW-CYTOMETRY-BASED SUPPRESSION ASSAY .............. 150

APPENDIX I

SUPPRESSIVE PROPERTY OF ACTIVATED CD4+CD25- T CELLS REVEALED BY A NOVEL FLOW CYTOMETRIC SUPPRESSION ASSAY

SUPPRESSIVE ACTIVITY CORRELATES WITH ACTIVATION STATUS AND CD25 EXPRESSION....

FOXP3 EXPRESSION IN ACTIVATED CD4+CD25- T CELLS IS NOT DUE TO TGF-B PRESENT IN HUMAN SERUM OR THAT PRODUCED BY ACTIVATED T CELLS OR APC

SUPPRESSIVE PROPERTY OF ACTIVATED T CELLS IS CONTACT DEPENDENT AND IS NOT DEPENDENT UPON IL-2 CONSUMPTION, COMPETITION FOR APC OR NUTRIENTS IN MEDIA 164 ANTIGEN NAÏVE AND MEMORY T CELLS EXPRESS FOXP3 UPON ACTIVATION AND ACQUIRE SUPPRESSIVE PROPERTIES . 168 
EFFECT OF KNOCKING OUT FOXP3 EXPRESSION IN HUMAN T

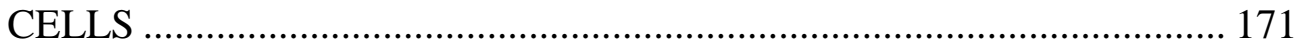

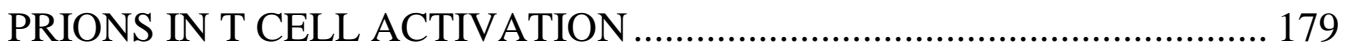

PHYSIOLOGICAL ROLE OF CELLULAR PRION PROTEIN ............... 181

PRPC IS EXPRESSED UBIQUITOUSLY BY HEMATOPOETIC CELLS

AND HIGHEST IN A SUBSET OF ACTIVATED/REGULATORY T

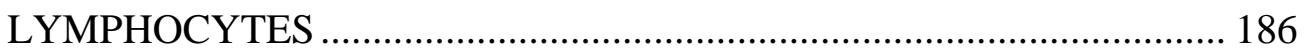

EX VIVO CD25+ T CELLS EXPRESS HIGHER LEVELS OF CELLULAR

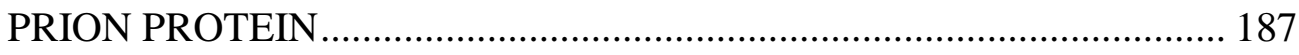

AFTER ACTIVATION, FOXP3+ CD4+CD25+ T CELLS EXPRESSED HIGHER LEVEL OF PRION PROTEIN ………….............................. 188

THE ROLE OF T CELLS IN TRANSPLANTATION ................................... 189

THE ROLE OF CD8+ TREGS IN TRANSPLANT …………………….... 193

CD8+ TREG SUPPRESS AUTOLOGOUS AND ALLOGENEIC ALLOREACTIVE CD4+CD25(-) T CELLS IN THE BOTH DIRECT AND INDIRECT ANTIGEN PRESENTATION PATHWAYS .......................... 197

APPENDIX II: PERSONAL RECORD ……………................................... 200

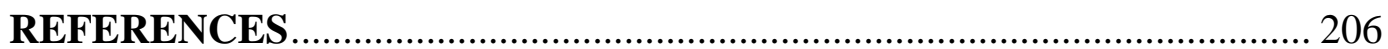

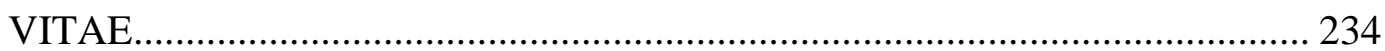




\section{PRIOR PUBLICATIONS AND ABSTRACTS}

Baughman EJ, Mendoza JP, Ortega SB, Ayers CL, Greenberg BM, Frohman EM, Karandikar NJ. Neuroantigen-specific CD8+ regulatory T-cell function is deficient during acute exacerbation of multiple sclerosis. J Autoimmun. 2011 Mar;36(2):115-24. Epub Jan 22, 2011. http://www.ncbi.nlm.nih.gov/pubmed/21257291

Vinodh Pillai, Nitin Karandikar, Ethan Baughman, Jason Mendoza, Imran Mohiuddin. Activation Induced Suppressive Ability in Human T Cells Revealed by Flow Cytometric Suppression Assay. Clinical Immunology, Volume 135, Supplement 1, 2010, Page S121. May 7, 2010. http://dx.doi.org/10.1016/j.clim.2010.03.363

Ethan Baughman, Jason Mendoza, Elliot Frohman, Nitin Karandikar. Autoreactive Regulatory CD8 $+\mathrm{T}$ Cells in Multiple Sclerosis. Clinical Immunology, Volume 135, Supplement 1, 2010, Pages S44-S45. May 7, 2010. http://dx.doi.org/10.1016/j.clim.2010.03.136

Ethan Baughman, Vinodh Pillai, Elliot Frohman, Nitin J Karandikar. Immune Regulatory Role for CNS-Specific, Autoreactive CD8+ T-cells in Multiple Sclerosis. 8th Course of the European School of Neuroimmunology. J Neuroimmunology Vol. 203, Issue 2, p 257. October 25, 2008. http://dx.doi.org/10.1016/j.jneuroim.2008.08.001

Vinodh Pillai, Ethan Baughman, and Nitin Karandikar. Suppressive Property of Activated FOXP3 + T Cells Revealed by a Novel Suppression Assay. Clinical Immunology Volume 127, Supplement 1, Page S50. April 16, 2008. http://dx.doi.org/10.1016/j.clim.2008.03.134

Ethan Baughman, Vinodh Pillai, Elliot Frohman and Nitin Karandikar. Immune Regulatory Role for CNS-specific, Autoreactive CD8 T Cells in Multiple Sclerosis. Clinical Immunology Volume 127, Supplement 1, Page S47. April 16, 2008 http://dx.doi.org/10.1016/j.clim.2008.03.124

Buseman CM, Tamura P, Sparks AA, Baughman EJ, Maatta S, Zhao J, Roth MR, Esch SW, Shah J, Williams TD, Welti R. Wounding stimulates the accumulation of glycerolipids containing oxophytodienoic acid and dinor-oxophytodienoic acid in Arabidopsis leaves. Plant Physiol. 2006 Sep;142(1):28-39. Epub Jul 14, 2006. http://www.ncbi.nlm.nih.gov/pubmed/16844834

Devaiah SP, Roth MR, Baughman E, Li M, Tamura P, Jeannotte R, Welti R, Wang X. Quantitative profiling of polar glycerolipid species from organs of wildtype Arabidopsis and a phospholipase D alpha1 knockout mutant. Phytochemistry. 2006 Sep;67(17):1907-24. Epub Jul 14, 2006. http://www.ncbi.nlm.nih.gov/pubmed/16843506 


\section{LIST OF FIGURES}

Figure 1: Thymic selection diminishes the number of self-reactive $T$ cells in the periphery

Figure 2: Immune regulatory/tolerance mechanisms may fail in certain, genetically-susceptible individuals, when exposed to environmental risk factors . 5 Figure 3: $\mathrm{T}_{\mathrm{REG}} \mathrm{S}$ are essential for controlling immune responses to self and contracting the immune response after clearance of foreign antigen .................... 11

Figure 4: CD4+ T cell differentiation ............................................................. 16 Figure 5: $\mathrm{T}$ cells become activated in the periphery and cross the blood-brain barrier 21

Figure 6: Demyelination syndrome subtypes are classified by clinical progression of neurological disability ................................................................................ 23

Figure 7: Axial luxol-fast blue staining of cervical spinal cord............................ 25

Figure 8: The prevalence of MS is increased at higher latitudes ........................... 30

Figure 9: Growing antigen-specific T cell lines ................................................. 74

Figure 10: Multiple sclerosis patients and healthy control subjects share similar T-

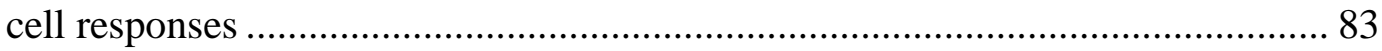

Figure 11: Multiple sclerosis patients and healthy control subjects share similar Tcell responses

Figure 12: Depletion of CD8+ T cells and CD25+ cells increased CD4+ T-cell responses to neuroantigens, but not to control foreign antigens ........................... 87

Figure 13: Basic design and gating strategy of flow-based suppression assay..... 90 Figure 14: Flow-based suppression Assay is validated: CD4+CD25+ Tregs and CD8+ T-cells suppress anti-CD3-stimulated CD4+ T-cells, but not CD4+CD25cells

Figure 15: Flow-based suppression Assay is validated: CD4+CD25+ Tregs and CD8+ T-cells suppress anti-CD3-stimulated CD4+ T-cells, but not CD4+CD25-

cells

Figure 16: Anti-CD3-stimulated and neuroantigen-specific CD8+ T-cells suppress

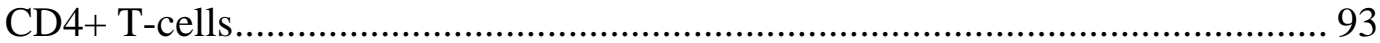

Figure 17: Neuroantigen-specific CD8+ T-cells suppress CD4+ T-cells............. 95

Figure 18: Neuroantigen-specific CD8+ T-cells suppress CD4+ T-cells............. 96

Figure 19: Antigenic specificity of $\mathrm{T}$ cell lines ..................................................... 98

Figure 20: Activated neuroantigen-specific CD8+ T-cells suppress CD4+ T-cells

Figure 21: Neuroantigen-specific suppressive ability is deficient during acute MS exacerbation

Figure 22: Neuroantigen-specific suppressive ability is deficient during acute MS

exacerbation

Figure 23: Average neuroantigen-specific suppressive ability is deficient during acute MS exacerbation.

Figure 24: Global CD8+ T cell suppressive ability is deficient during acute MS exacerbation 105

Figure 25: Foreign-specific suppressive ability is unchanged during acute MS exacerbation 
Figure 26: During suppression assays, MS and healthy controls share similar $\mathrm{CD} 8+\mathrm{T}$ cell activation to neuroantigen

Figure 27: During suppression assays, MS and healthy controls share similar $\mathrm{CD} 8+\mathrm{T}$ cell activation to foreign antigen

Figure 28: During suppression assays, MS and healthy controls share similar $\mathrm{CD} 8+\mathrm{T}$ cell activation to anti-CD3

Figure 29: Neuroantigen-specific CD8+ T-cell suppressive-ability correlates with days since last relapse.

Figure 30: Foreign antigen-specific CD8+ T-cell suppressive-ability does not

correlate with days since last relapse.

Figure 31: Global CD8+ T-cell suppressive-ability does not strongly correlate with days since last relapse

Figure 32: Neuroantigen-specific CD8+ T-cell suppressive-ability correlates with days since last relapse and recovery

Figure 33: Mean neuroantigen-specific CD8+ T-cell suppression decreases

transiently during acute MS exacerbation.

Figure 34: Foreign antigen-specific CD8+ T-cell suppressive-ability does not correlate with days since last relapse and recovery

Figure 35: Global CD8+ T-cell suppressive-ability does not correlate with days

since last relapse and recovery.

Figure 36: Neuroantigen-specific CD8+ T-cell suppression decreases transiently

during acute $\mathrm{MS}$ exacerbation

Figure 37: CNS-Specific CD8+ T cells require HLA class I, not TNF, in order to suppress CNS-specific CD4+ T cells.

Figure 38: Neuroantigen-specific suppression by CD8+ T cells was dependent upon HLA class I, IFN $\gamma$, with possible partial involvement by NKG2D, PD-1, and IL10....

Figure 39: CNS-specific suppression is harbored in the CD8+CD62L(-) subset 126 Figure 40: GA-specific suppression is harbored in the CD8+CD62L(-) subset. 127 Figure 41: Global CD8 suppressive ability is harbored in the CD28+ subset in adults, in contrast to neonates. CD8+ subset harboring non-specific suppression changes over a lifetime

Figure 42: Activated neuroantigen-specific CD8+ T-cells suppress CD4+ T-cells

Figure 43: Model of how CNS-specific regulatory ability is directly or indirectly involved in the mechanism of MS clinical phase change....

Figure 44: CD8+ cells having the most suppressive ability may include terminally differentiated or exhausted CD8+ T cells

Figure 45: The adaptive immune system is not shared by organisms lower than

jawless vertebrates, which lack the recombinase-activating gene 153 Figure 46: Novel flow cytometry based suppression assay uses differential staining of sorted populations to isolate and measure responder proliferation... 158 Figure 47: Naturally-occurring CD4+CD25+ Tregs and activated FOXP3expressing ex vivo CD4+CD25- T cells suppressed while non-activated ex vivo CD4+CD25-FOXP3- did not suppress autologous ex vivo CD4+CD25responders 
Figure 48: FOXP3 expression by activated T cells is not due to presence of TGF $\beta$ in serum or that secreted by activated $\mathrm{T}$ cells .................................................. 163 Figure 49: Compared to nTreg, suppressive activity of activated CD4+CD25- T cells was increased and not affected by addition of anti-TGF $\beta$ or anti-IL-10 or high dose IL-2 166

Figure 50: Suppressive activity of activated CD4+CD25- T cells is contact

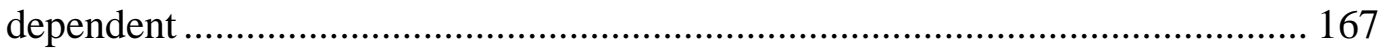
Figure 51: Cord blood naïve T cells express FOX3 upon activation by plate bound anti-CD3/anti CD28 in serum free X-vivo media. 169 Figure 52: Treg-depleted CD4+CD25- T cells were resistant to puromycin treatment after shRNA-lentivirus infection 172

Figure 53: FOXP3 shRNA lentivirus achieves partial protein knockdown of FOXP3

Figure 54: Polybrene-treated iTreg suppressive ability is not detectable by flow-

based suppression assay. 174

Figure 55: PrPc is expressed ubiquitously by hematopoetic cells and highest in a subset of activated/regulatory $\mathrm{T}$ lymphocytes 186 Figure 56: Ex vivo CD25+ T cells express higher levels of cellular prion protein.

Figure 57: After activation, Foxp3+CD4+CD25+ T cells expressed higher level of Prion Protein 188

Figure 58: Alloantigen-presentation pathways 196 Figure 59: Alloantigen-specific CD8+ Treg suppress autologous and allogeneic alloreactive CD4+CD25(-) T cells in the both direct and indirect antigen presentation pathways. 198

Figure 60: MS patients have deficient CD4 and CD8 Treg function 199 


\section{LIST OF TABLES}

Table 1: Summary of Patient Characteristics...................................................... 69

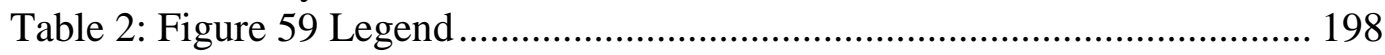




\section{LIST OF ABBREVIATIONS}

ADEM (acute disseminated encephalomyelitis)

APC (antigen-presenting cells)

$B$ cell (bone marrow-derived lymphocyte)

BBB (blood-brain barrier)

BDNF (bone-marrow derived neurotrophic factor)

CD4/8/25+ (cluster of differentiation $4,8,25$, et cetera positive)

CNS (central nervous system)

CNTF (cytokine ciliary neurotrophic factor)

CSF (cerebrospinal fluid)

CTLA4 (cytotoxic T-lymphocyte antigen 4)

DMA (disease-modifying agents)

EAE (experimental autoimmune encephalomyelitis)

EBV (Epstein-Barr virus)

FDA (food and drug administration)

FOXP3 (forkhead box P3)

GA (copolymer-1/glatiramer acetate/Copaxone ${ }^{\circledR}$ )

$\mathrm{H} \& \mathrm{E}$ (hematoxylin and eosin)

HHV-6 (human herpes virus 6)

HLA (human leukocyte antigen)

IDO (indoleamine-pyrrole 2,3-dioxygenase)

IFNAR (interferon $-\alpha / \beta$ receptor)

IFN $\beta$ (type-one interferon beta)

IFN $\gamma$ (interferon gamma)

IFN $\gamma \mathrm{R}$ (IFN $\gamma$ receptor)

Ig (immunoglobulins)

IL10/15 (interleukin 10, 15, et cetera)

IL27R (IL27 receptor)

IL2R $\alpha$ (interleukin 2 receptor alpha)

iNOS (inducible nitric oxide synthase)

IPEX (immunodysregulation polyendocrinopathy enteropathy X-linked syndrome)

IRF1 (interferon regulatory factor 1)

IVIG (intravenous Ig)

MAG (myelin-associated glycoprotein)

MBP (myelin basic protein)

MHC (major histocompatibility complex)

MMP (matrix metalloproteases)

MOG (myelin oligodendrocyte glycoprotein)

MRI (magnetic resonance imaging)

MS (multiple sclerosis)

MSRV (endogenous multiple sclerosis-associated retrovirus)

MX (mitoxantrone)

Myd88 (myeloid differentiation primary response gene 88)

PBMC (peripheral blood mononuclear cells) 
PD-1 (programmed death 1)

PLP (proteolipid protein)

PML (progressive multifocal leukoencephalopthy)

PPD (Purified protein derivative of tuberculin)

PPMS (primary progressive MS)

RAG (recombinase-activating genes)

ROR $\gamma \mathrm{t}$ (retinoic acid receptor-related orphan receptor gamma isoform $\mathrm{t}$ )

RRMS (relapsing-remitting MS)

SNP (single nucleotide polymorphism)

SPMS (secondary or chronic progressive MS)

STAT1(signal transducers and activators of transcription protein 1)

steroids (corticosteroids)

T cell (thymus-derived lymphocyte)

T1DM (type one diabetes mellitus)

TBET (Th1-specific T box transcription factor)

TCR ( $\mathrm{T}$ cell receptor)

Th1 (CD4+ helper type 1)

Th17 (CD4+ helper type 17)

Th2 (CD4+ helper type 2)

TNFRII (tumor necrosis factor receptor 2)

Treg (suppressor/regulatory T cell)

US (United States)

VCAM (vascular adhesion molecule)

VLA4 (very late antigen-4) 


\section{CHAPTER 1: INTRODUCTION}

\section{TOLERANCE}

Conceptually, two major regulatory mechanisms exist to guard against immune-related pathology. Central tolerance and peripheral tolerance mechanisms maintain the distinction between self and non-self.

\section{CENTRAL TOLERANCE}

Central tolerance mechanisms occur during thymic selection [5]. In the thymus, thymocytes, expressing a range of randomly-generated TCRs, undergo positive and negative selection (Figure 1). The thymus is most active during fetal life and atrophies with increasing age. Based on the specificity of the TCR, thymocytes are selected which bind weakly to HLA and do not strongly react with self. Thymocytes that do not bind HLA die of neglect, and thymocytes that strongly react with self are clonally deleted in an active manner. Clonal deletion in the thymus centrally promotes tolerance to self before educated thymocytes immigrate to the periphery and also promotes a repertoire of TCRs directed toward pathogens. The number of self-reactive $\mathrm{T}$ cells in the periphery is diminished compared to the pre-thymic selection pool. 
Figure 1: Thymic selection diminishes the number of self-reactive $T$ cells in the periphery

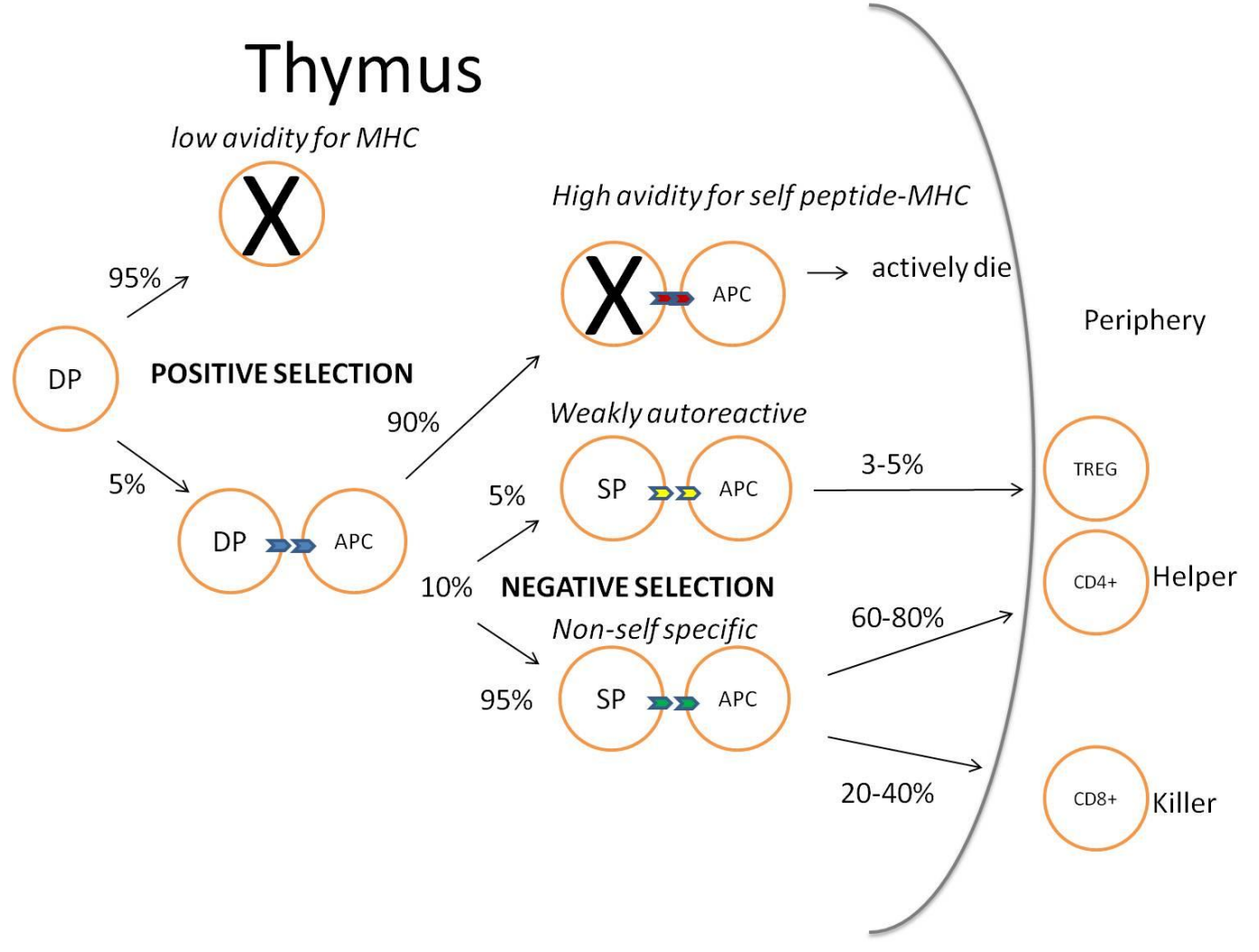




\section{PERIPHERAL TOLERANCE}

Mechanisms of peripheral tolerance deal with autoreactive $T$ cells in the periphery [6]. Self-reactive T cells that survive thymic selection are detectable in the periphery and have lower TCR avidity for HLA compared to foreign antigenspecific counterparts. Limited numbers of self-reactive $\mathrm{T}$ cells in the periphery may serve a physiological function or cause autoimmunity if reactivated with inappropriate signals. Regulatory $\mathrm{T}$ cells may serve to counter balance the pathogenic potential of autoreactive $\mathrm{T}$ cells. Untoward immune responses are thought to result in absence of regulatory $\mathrm{T}$ cells. Through a puzzling mechanism, regulatory $\mathrm{T}$ cells may express low-avidity autoreactive TCRs. Autoimmunity may result from the dysfunctional conversion of autoreactive $\mathrm{T}$ cells away from a regulatory phenotype. Autoreactive, myelin-specific $\mathrm{T}$ cell responses are prevalent in healthy person and multiple sclerosis (MS) patients. Antigen exposure in the periphery and the lack of tolerogenic context of the encounter presumably are the critical immunological events in persons diagnosed with MS.

\section{AUTOIMMUNITY}

Approximately 1 in 31 Americans lives with an autoimmune disease [7]. The immune responses against self are often mediated by antigen-specific $\mathrm{T}$ cells, but controlled peripherally by a proper balance of Tregs (Figure 2). The experimental removal of the Treg subset can result in a variety of organ-specific autoimmune diseases [8]. Lethal autoimmune syndromes are characteristic phenotypes of several knockout mice: IL-R $\alpha$ (CD25), IL-R $\beta$ (CD122) [9], 
forkhead transcription factor FOXP3 [10], and TGF $\beta 1$ [11]. The prevention of immune attack against autologous cells by adoptive transfer of Treg is the subject of numerous studies. Furthermore, adoptive transfer of CD25-depleted T cells into thymectomized mice results in general immune dysregulation and autoimmunity [12-14]. Defects in regulatory $\mathrm{T}$ cell function have been demonstrated in a wide variety of human disease [15]. In nearly all such cases, it is unclear whether an intrinsic defect lies in the ability of Tregs to suppress or in responder T cell hyporesponsiveness to Treg [16]. However, ex vivo generated and in vivo induced Treg can mediate therapy for T1DM [17-21]. A deficiency in the transcription factor FOXP3 is thought to result in defective regulatory $\mathrm{T}$ cell development and causes IPEX syndrome in humans [22]. IPEX neonates display T1DM, enteropathy, and endocrinopathy. The mouse form of disease, the scurvy mouse, exhibits a shortened lifespan, multiorgan inflammation, eosinophilia, cytokine storm, and hyperimmunoglobinemia. FOXP3 activity is regulated by TCR, CD3, CD28, CTLA4, TGF $\beta$, PGE2, and others. FOXP3 is a global immune regulator, having modulatory effects on IL4, IL2, IL5, IL2RA, IgG2a, IgM, IgG1, and CD40LG [23]. 
Figure 2: Immune regulatory/tolerance mechanisms may fail in certain, genetically-susceptible individuals, when exposed to environmental risk factors

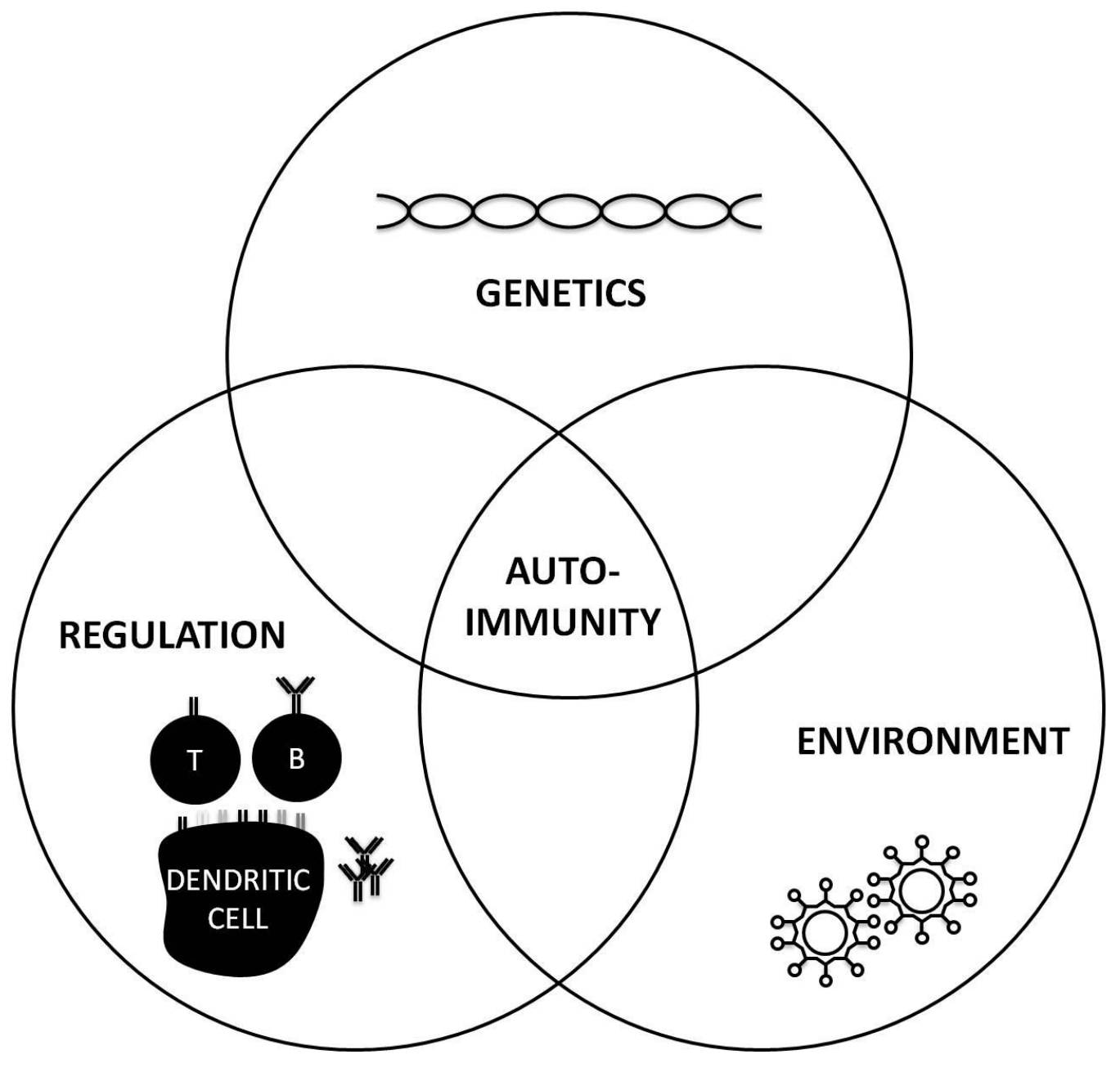




\section{SUPPRESSOR T CELLS}

The first report of a $\mathrm{T}$ cell population capable of suppressing other $\mathrm{T}$ cells was made some forty years ago while studying interactions between thymocytes in lethally irradiated mice lacking B cells [24]. The suppression assay measured total in vivo proliferative response to antigenic stimulation with sheep red blood cells (SRBC) by individual populations of T cells and combinations thereof. The proliferative response of primed (responder) cells was greater than the combined total proliferative response of primed (responder) cells plus unprimed (suppressor) $\mathrm{T}$ cells, suggesting that the unprimed (suppressor) $\mathrm{T}$ cells were capable of suppressing SRBC antigen-specific T cell responses.

A similar observation was also made using adoptive transfer of allogeneic (responder) cells into lethally irradiated hosts with and without autologous (suppressor) cells. Namely, the proliferative response of allogeneic (responder) cells was greater than the combined total proliferative response of allogeneic (responder) cells plus autologous (suppressor) host cells, suggesting that host cells could suppress graft-versus-host disease (GVHD)-causing alloantigen-specific $\mathrm{T}$ cell responses. These two important instances of antagonistic interactions between $\mathrm{T}$ cell populations highlighted the importance of suppressor $\mathrm{T}$ cells $(\mathrm{Ts})$ for the first time. Competition for space in the spleen was thought to be unlikely as the authors had determined by other adoptive transfer experiments that the spleen's capacity was significantly higher than the cell numbers used in the suppression experiments. The authors hypothesized some cellular interaction was responsible for the suppression. 
If the addition of unprimed $\mathrm{T}$ cell populations resulted in reduced total primed plus unprimed combined $\mathrm{T}$ cell proliferation, scientists thought that the removal of the source of unprimed $\mathrm{T}$ cells may increase $\mathrm{T}$ cell proliferation. The assertion turned out to be correct. Thymectomized animals were found to spontaneously develop oophoritsis, thyroiditis and auto-antibodies to thyroglobulin $[25,26]$. Mice thymectomized between the second and fifth day of life have auto-immune reactions to multiple organs. CD4+ T cells infiltrate the affected organs, and auto-antibodies are generated [27]. CD90+CD5+ thymocytes were capable of adoptively transferring post-thymectomy autoimmune oophoritis [28]. Post-thymectomy autoimmunity was thought to develop as a result of depleted newly generated $\mathrm{T}$ lymphocytes. Normal lymphoid cells from euthymic mice transferred into athymic mice could prevent autoimmunity [29]. It remained unclear if other $\mathrm{T}$ populations were capable of suppressing $\mathrm{T}$ cells.

Other experiments showed that both CD4+ and CD8+ $\mathrm{T}$ cells were capable of suppression through unknown mechanisms. It was known that small amounts of antigen given orally inhibited immune responses upon re-exposure. Myelin basic protein (MBP), given orally, suppressed experimental autoimmune encephalomyelitis (EAE) through the transforming growth factor beta (TGF $\beta$ )producing CD8+ T cells [30]. Hence, soluble cytokines were thought to mediate suppression. The surface phenotype of suppressor $\mathrm{T}$ cells was even more enigmatic. Thought to bear the CD8 marker, similar to cytotoxic T lymphocytes (CTL), the expected mechanism of suppression was thought to be cell-mediated 
cytolytic ability. A few distinctions were thought to identify CD8+ suppressors from killers. CTLs were CD45RA+IL10(-) and lacked the ability to suppress Th1 and Th2 cells [31]. Suppressor CD8+ cells expressed IL10 and IFN $\gamma$ and suppressed the proliferation of CD4+ cells.

\section{I-J PARADOX}

Perhaps the most contentious issue in the history of the suppressor $\mathrm{T}$ cell relates to the I-J paradox, a discrepancy between serological mapping of a presumed protein(s) called I-J and molecular genetic mapping [32]. Limited molecular technology limited the study of T suppressors in the 1970s and 1980s [33]. Early genetic linkage studies and Mendelian segregation experiments estimated the number and layout of the MHC loci on chromosome $17[34,35]$. Suppressor T cells were thought to express an I-J molecule hypothetically located in the I-C region. In his shared 1980 Noble prize lecture in physiology or medicine "for their discoveries concerning genetically determined structures on the cell surface that regulate immunological reactions", Baruj Benacerraf described the presumed role of I-J in the suppressor T cell circuits. When the I region between the $\mathrm{K}$ and $\mathrm{D}$ loci was finally cloned and sequenced, no I-J gene could be identified in the local area [36]. Some thought that the I-J determinant was linked to MHC but the genetically mapped location was incorrect, possibly due to high frequency of multiple crossovers. The I-J paradox was never resolved and caused many immunologists to be skeptical of immune-regulatory phenomena and theory, including suppressor $\mathrm{T}$ cells. The old notions of the suppressor $\mathrm{T}$ network fell out of favor and became subject to necessary and 
unnecessary criticism. For a time, discussion of suppression became synonymous with over-interpretation of scanty data. While much of the non-contentious work of these investigators has been forgotten or under-appreciated, past and recent evidence strongly supports the role for $\mathrm{T}$ cell mediated suppression in maintenance of peripheral tolerance.

\section{REGULATORY T CELLS}

Circulating throughout the blood and lymph are CD8+ and CD4+ T cells with suppressor potential (Figure 3). Suppressor cells (Ts) and regulatory T cells $\left(\mathrm{T}_{\mathrm{REG}}(\mathrm{s})\right)$ are used synonymously here. The better-accepted and -understood variety of human $\mathrm{T}_{\mathrm{REG}} \mathrm{S}$ is thought to develop "naturally" from thymocyte selective processes. These natural Tregs $\left(\mathrm{nT}_{\mathrm{REG}} \mathrm{S}\right)$ are defined by their constitutive expression of the transcription factor FOXP3 and also express high levels the alpha chain of the IL2 receptor (CD25). Approximately 5-10\% of CD4+ T cells that emigrate from the thymus into the blood stream exhibit the FOXP3+CD25+ regulatory phenotype. While much is known about CD4+ $\mathrm{T}_{\mathrm{REG}} \mathrm{S}, \mathrm{CD} 8+\mathrm{T}_{\mathrm{REG}} \mathrm{S}$ remain understudied. Tolerance to self antigen by Treg is crucial to prevent autoimmune tissue damage. In the context of hematopoetic or solid tissue transplant, graft rejection may be slowed or prevented through the use of donor antigen-specific $\mathrm{T}_{\mathrm{REG}} \mathrm{S}$. $\mathrm{T}_{\mathrm{REG}} \mathrm{S}$ control amplified immune responses to innocuous foreign antigens and prevent allergic/atopic pathology. While most studies in the context of transplant implicate the CD28(-) subset of CD8+ T cells as having the most relevant regulatory potential [37-43], other studies point to other regulatory CD8+ T cells restricted to HLA-E [44], $\gamma \delta$-TCR CD8+ T cells [45], or CD8+ 
subsets expressing CD25 [46], CD122 [47, 48], CD103 [49] [50], GITR [51, 52], PD-1 [53] or FOXP3 [54] [55] [56]. The most well understood regulatory CD8+ $\mathrm{T}$ cells kill autoreactive, pathogenic CD4+ T cells that express HSP60 peptideHLA-E complexes [44]. Naturally-occurring CD8+CD122+ T cells produce IL10 and can only suppress autologous MHC-I-expressing T cell-IFN $\gamma$ production in a Qa-1-independent manner. Alloantigen-specific CD8+CD28(-) $\mathrm{T}_{\mathrm{REG}}$ suppress through upregulating ILT3 and ILT4 [54]. Human TEG $_{\text {RE suppressive }}$ function can be abrogated with the use of TLR8 agonists [45]. Antigen-specific regulatory $\mathrm{CD} 8+\mathrm{T}$ cells can be generated ex vivo $[46,57]$ and are capable of organ-specific immunosuppression. The phenomenon of $\mathrm{T}$ cells mediating suppression of immune responses has been observed for decades, while the mechanism(s) of such action has remained a topic of intense study and controversy. 
Figure 3: $\mathbf{T}_{\mathbf{R E G}} \mathrm{S}$ are essential for controlling immune responses to self and contracting the immune response after clearance of foreign antigen

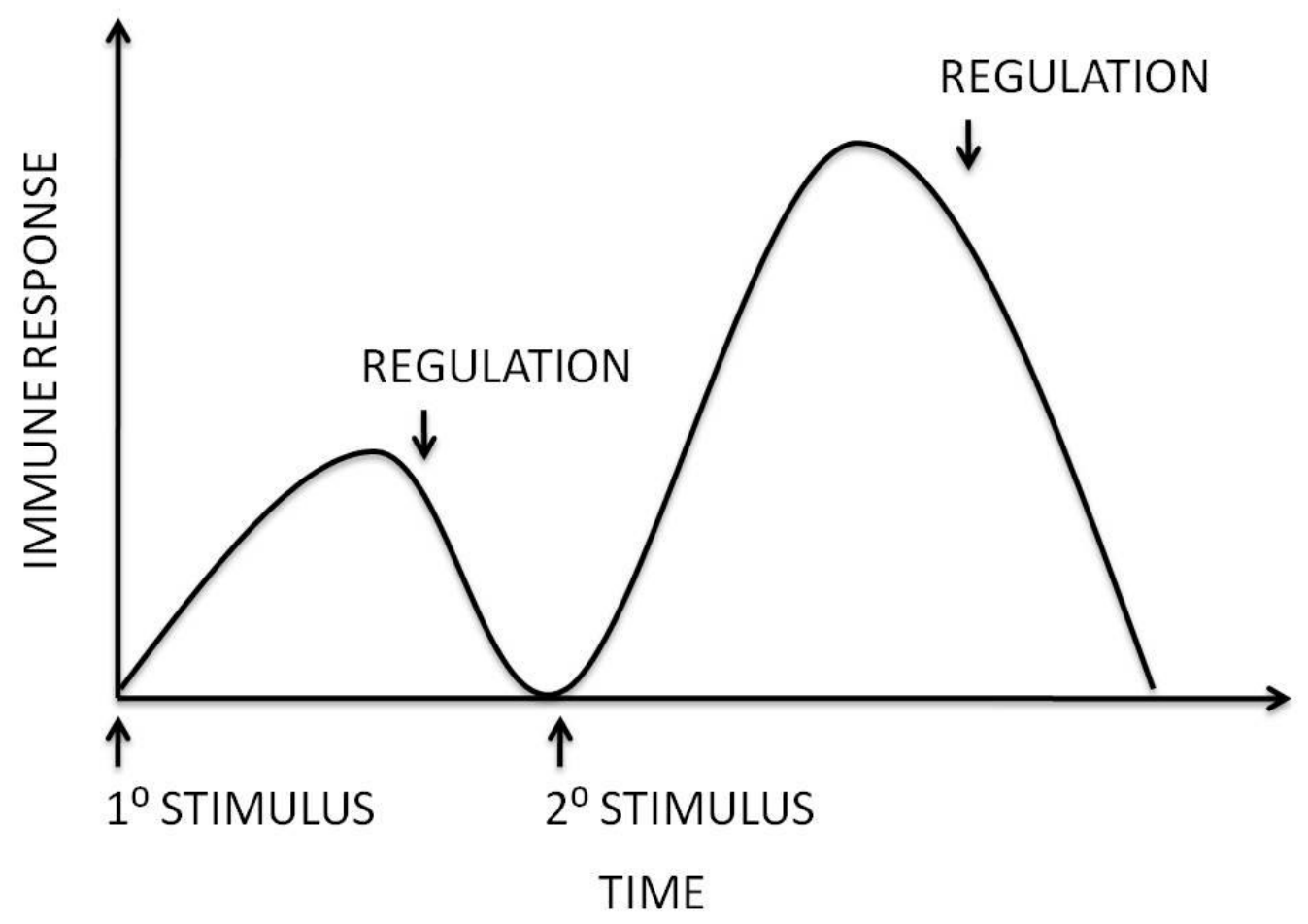




\section{ANTIGEN-SPECIFIC CD8+ TREGS}

Antigen-specific $\mathrm{CD} 4+$ and $\mathrm{CD} 8+\mathrm{T}_{\mathrm{REG}} \mathrm{S}$ are essential for controlling immune responses to self and contracting the immune response after clearance of foreign antigen [58]. Mice deficient in the CD8 molecule suffered clinically more severe EAE compared to wild-type mice. As a potential mechanism of peripheral tolerance, CD8+ T cells' TCRs specifically recognize a unique set of self-peptides presented by the MHC class Ib molecule Qa-1, HLA-E in humans, differentially expressed on T cells as a function of the affinity/avidity of T cell activation [59]. Challenging notions about the definition of an effector cytokine, antigen-specific $\mathrm{CD} 8+\mathrm{T}_{\mathrm{REG}} \mathrm{S}$ require IFN $\gamma$ for induction or suppressive function during viral infection, cancer, or MS therapy [60]. As a means of maintaining the immunologic privilege of the eye, anterior chamber-injected antigen induces CD8+ $\mathrm{T}_{\mathrm{REG}} \mathrm{S}$, which depend on CD94/NKG2A-Qa-1 interactions to reduce ocular DTH responses [61]. Ocular iris pigment epithelia constitutively express CD80/86 and membrane-bound active TGF $\beta$ to induce CD8+B7+ Tregs [62]. Injection of antigen into the anterior chamber of an eye induces splenic CD8+ T cells whose suppressor function requires IFN $\gamma$ receptor stimulation [63]. Antigen injection into the ocular anterior chamber induces CD8+CD103+ $\mathrm{T}_{\mathrm{REG}} \mathrm{S}$ [64]. The

context of antigen presentation has important consequences toward the generations of $\mathrm{T}_{\mathrm{REG}} \mathrm{S}$. Vasoactive intestinal peptide (VIP)-treated monocytes differentiate into toleragenic DC, which are capable of inducing IL-10-producing CD8+CD28-CTLA4+ T cells capable of suppressing antigen-specific Th1 cells [65]. 
In the context of autoimmune disease, $\mathrm{T}_{\mathrm{REG}} \mathrm{S}$ have been the subject of intense study and controversy. Present at a higher frequency in ankylosing spondylitis, autoreactive CD8+CD25+ FOXP3+CTLA-4+ T cells produce IL-4, IL-5, IL-13 and TGF $\beta$ and suppress CD4+ T cell proliferation and IFN $\gamma$ production in a CTLA-4- and HLA-I-dependent manner [66]. During MS exacerbations, CD8+ T cell clones express significantly more killer-inhibitory receptor $\mathrm{CD} 94 / \mathrm{NKG} 2 \mathrm{~A}$, which $\mathrm{IL}-15$ and IFN $\gamma$ further increase, and exhibit reduced cytolytic ability towards MBP- and MOG-specific CD4+ T cells [67]. Human CD8+ T cells, derived from CD45RA+ CD27- cells, suppress glutamic acid decarboxylase 65-specific CD4+ T cells in a contact- and IL-10-dependent manner [68]. The potential for therapeutic advantage from these and other studies will be discussed in further detail.

\section{GLOBALLY SUPPRESSIVE, NON-SPECIFIC CD8+ TREGS}

Polyclonal CD4+ and CD8+ Tregs have suppressive ability in several models of autoimmunity, transplant, and GvHD [69]. Antigen non-specific Type 2 CD8+ $\mathrm{T}$ suppressors, which inhibit $\mathrm{T}$ cell proliferation and are cytolytic by soluble factors, are deficient in multiple sclerosis, systemic lupus erythematosus, or systemic sclerosis as well as HIV or chronic HCV infected patients [70]. In $\mathrm{MHCII}^{-/}$mice, CD8+CD25+ T cells are GITR+CTLA+FOXP3+IL-10+, inhibit anti-CD3 stimulated CD25- T cells, and proliferated and produced IFN $\gamma$ as well [71]. The intestinal parasite, Heligmosomoides polygyrus, induced CD8+ T cells in the intestinal lamina propria having suppressive ability toward splenocyte antiCD3 stimulation [72]. Even in the steady-state, intraepithelial CD8+CD28- 
CD103+ $\mathrm{T}$ cells appear to possess suppressive function, and their absence may predispose to inflammatory bowel disease [73]. The phenomenon of non-specific $\mathrm{T}_{\mathrm{REG}}$ suppression suggests that entirely different mechanisms of action differentiate many distinct subsets of $\mathrm{T}_{\mathrm{REG}} \mathrm{S}$.

\section{T CELL ACTIVATION AND DIFFERENTIATION}

$\mathrm{T}$ cell activation and its context are generally thought to involve three external signals. The first signal is TCR stimulation. The second is costimulation, and the third signals are cytokines. $\mathrm{T}$ cell activation and differentiation is thought to be regulated by several families of signaling molecules and transcription factors. Upon TCR activation, CD3 and Lck signal to ZAP70 [74]. ZAP70 is phosphorylated, which signals to phospholipase $\mathrm{C} \gamma 1$ to generate inositol triphosphate (IP3) and diacylglycerol (DAG). IP3 causes calcium release intracellularly, which activates the nuclear factor of activated $\mathrm{T}$ cell (NFAT) pathway. DAG leads to the phosphorylation of protein kinase $\mathrm{C} \theta$, which activates the TCR-induced I $\mathrm{KB}$ kinase (IKK) and NF- $\kappa \mathrm{B}$ pathway. Stimulation of TCR or CD28 alone is insufficient to activate naïve T cells [75]. Co-stimulatory signals from the CD28 family are also needed to activate PDK1, which through Akt completely activates NF- $\kappa \mathrm{B}$. The third exterior signal, cytokines, is transmitted through cytokine receptors and the JAK/STAT pathway. While CD4+ T cell differentiation has been studied extensively (Figure 4), CD8+ $\mathrm{T}$ cell differentiation is poorly understood and may be incorrectly presumed to mirror CD4+ T cell differentiation. 
Naïve CD4+ T helper cells were once thought to differentiate into two subsets, Th1 and Th2, each with a distinct function and cytokine production profile [76]. IL12 signals through STAT4 to promote Th1 cell differentiation [77]. T-bet expression activates the Th1 genetic program that commits the $\mathrm{T}$ cell to producing IL-2 and IFN $\gamma$. Th1 cells are thought to have an important role in cell-mediated immunity. Th1 development attenuates the Th2 program and vice versa [78]. IL4 signals through STAT6 to promote Th2 cell differentiation. GATA3 expression activates the Th2 genetic program that commits the $\mathrm{T}$ cell to produce IL4, IL5, and IL13 [79]. Th2 cells are thought to have an important role in humoral-mediated immunity.

More recently, important roles for FOXP3 and retinoid-related orphan receptor (ROR) $\gamma$ in effector $\mathrm{T}$ cell differentiation have been recognized [80]. ROR $\gamma$ expression activates the Th17 genetic program that commits the $\mathrm{T}$ cell to producing IL17 [81]. Th17 cells are thought to have an important proinflammatory role in autoimmunity and gut homeostasis [82] [83]. FOXP3 expression is thought to activate the Treg genetic program that commits the $\mathrm{T}$ cell to produce TGF $\beta$ [84]. Tregs have an important role in down-regulating Th1, Th2, and Th17 cells [85] [86]. IL6 is thought promote either the Th17 or Treg lineage when combined with other cytokines [87]. Combined with IL6, TGF $\beta$ or IL23 promote the generation of Treg or Th17 cells, respectively [88] [89]. While many of the master regulatory transcription factors of $\mathrm{T}$ cells have been worked out in CD4+ $\mathrm{T}$ cells, differentiation and lineage commitment of CD8+ $\mathrm{T}$ cell remains less clear. 
Figure 4: CD4+ $\mathrm{T}$ cell differentiation

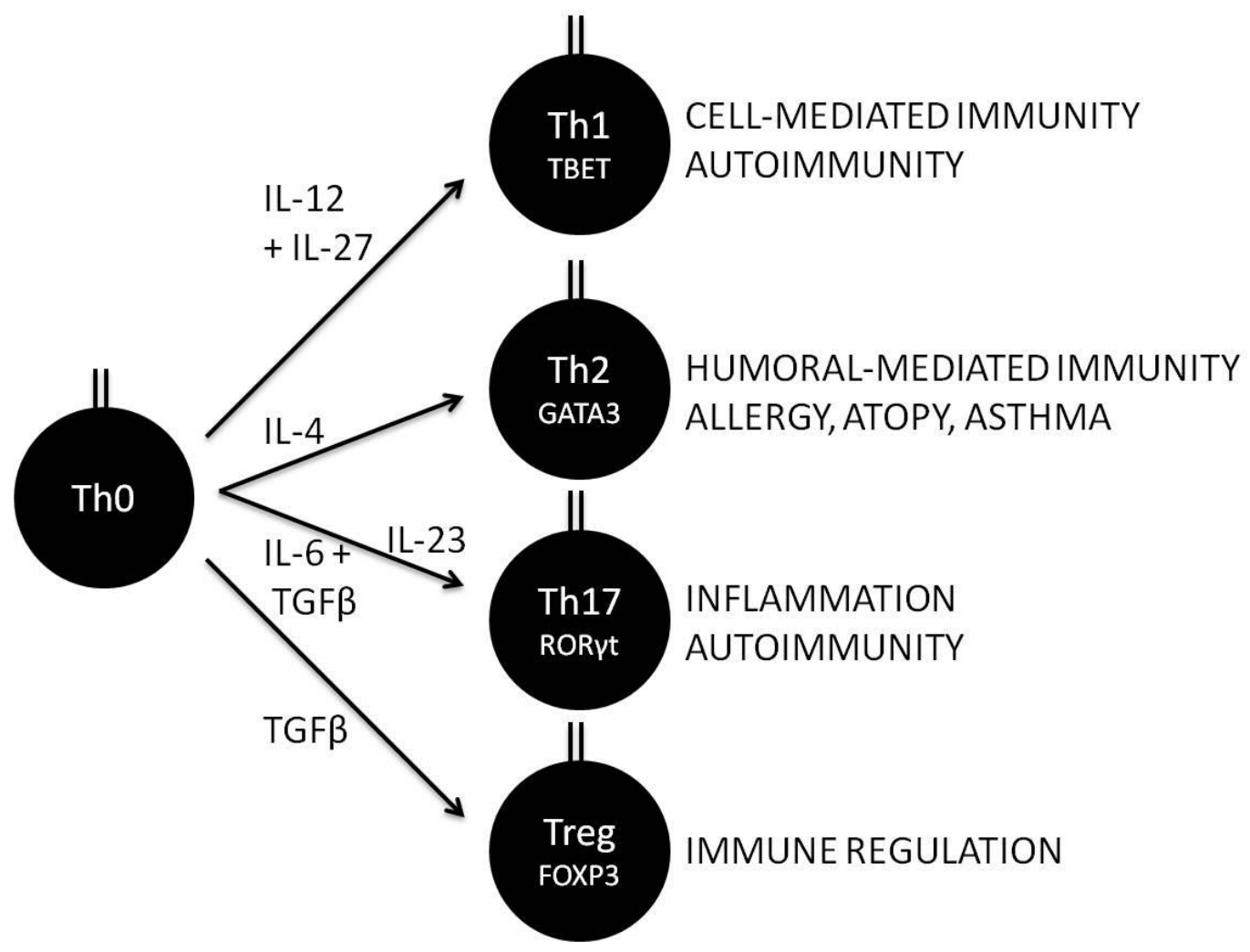




\section{TRANSCRIPTIONA.L REGULATION OF TREG}

The roles of master regulator transcription factors that globally regulate $\mathrm{T}$ cell gene expression are still under investigation. FOXP3 was recently recognized as a master regulator of $\mathrm{T}$ cell gene expression. FOXP3+CD25+ $\mathrm{T}$ cells downregulate or suppress untoward immune responses by other non-FOXP3 $+\mathrm{T}$ cell populations, playing a role in autoimmunity, transplant, allergy, tumors, and infections. CD4+CD25+FOXP+ cells are the most well accepted phenotype of CD4+ Tregs. CD8+CD25+FOXP3+ $\mathrm{T}$ cells can be generated by continuous antigen stimulation, which induces upregulation of costimulatory and cytotoxic molecules, enabling inhibition of $\mathrm{CD} 4+$ and $\mathrm{CD} 8+\mathrm{T}$ cell proliferation and cytokine production, independent of CTLA4, CD80/86, prostaglandin E(2), IL-10 and TGF-beta, yet in a contact-dependent manner [46]. PPD-primed CD8+CD25+FOXP3+ Tregs suppressed live bacillus Calmette Guérin-specific responses and produced CCL4 [90]. CD8 + CD28- T cells, which express FOXP3, are expanded in blood of lung cancer patients [55]. The FOXP3 aspect of peripheral tolerance is important for contracting the immune response and lessening the activity of potentially harmful autoreactive T cells. While FOXP3 is thought to be the most specific marker known for human regulatory $\mathrm{T}$ cells (Tregs), in vitro suppressive activity remains the benchmark for any lineage of regulatory T cells. Natural Tregs are derived from the thymus. Adaptive Tregs are induced in the periphery; FOXP3 expression is induced by virtually any stimulus that activates T cells. Both CD8+FOXP3(-) and CD4+FOXP3(-) human T cells transiently express FOXP3 and CD25 upon activation. While T cells 
attain the $\mathrm{FOXP} 3+\mathrm{CD} 25+$ status, the cells inhibit in vitro proliferation of autologous $\mathrm{T}$ cells, suppressing IFN $\gamma$ production by responders cells by expressing TNF $\alpha$ and IL10 [91]. Activation-induced FOXP3 expression is downregulated along with suppressive ability in all but a fraction of circulating $\mathrm{T}$ cells; the FOXP3+ remainder are possibly the source of de novo peripherally induced Tregs. Activated FOXP3 expressing $\mathrm{T}$ cells may regulate excessive immune activation in vivo. FOXP3+ $\mathrm{T}$ cells may represent a plastic and dynamic population, potentially informed by the pool of activated $\mathrm{T}$ cells. It remains unclear what effector function remains armed in FOXP3 $+\mathrm{T}$ cells and how much clinical potential Tregs possess.

\section{SUPPRESSIVE PROPERTY OF ACTIVATED FOXP3+ T CELLS}

Whether activated human T-cells that express FOXP3 have regulatory properties is a subject of intense study and controversy. While a few groups have previously shown suppressive activity in activated T-cells [91], many other studies have concluded that activated/induced $\mathrm{FOXP}^{+}{ }^{+}$-cells do not acquire suppressive properties at all or acquire it only if there is sustained FOXP3 expression. There are several possible reasons for such disparate results. It may be the use of an insensitive suppression assay, using bulk cultures of activated cells measured by total tritiated thymidine uptake of all cells in the culture. The insensitivity of the assay may be explained by impure suppressors cells contaminated by non-anergic responders cells. Suppressive property of the regulatory population may be masked by the proliferation of the other non-anergic cells in the mix. Suppressor cells may also not be completely anergic. To cope 
with these and other challenges, we devised a novel flow cytometry-based suppression assay (described in the methods and results), whereby the putative suppressor cells were excluded from the analysis of proliferation, avoiding the pitfalls of the thymidine-based assay through taking advantage of proliferation dyes and long-term cellular tracking dyes. 


\section{IMMUNE-MEDIATED DYMELINATION}

The preponderant belief is that multiple sclerosis (MS) and experimental autoimmune encephalomyelitis (EAE) are mediated through central nervous system (CNS)-specific CD4+ helper type 1 (Th1) and type 17 (Th17) responses and regulated by CD4+ regulatory T-cells [92]. Considerable evidence, however, points to an important role for CD8+ T-cells in the pathogenesis and/or regulation [93] of MS and EAE [94, 95] [96] [97] [98] [99] [100] [101] [44] [17, 102, 103] $[47,104,105]$ [106]. While granzyme B-expressing CD8+ T cells predominate in demyelinated areas of acute MS lesions, CD8+CD122+ T cells also regulate the recovery phase of EAE, as shown through adoptive transfer and anti-CD122 mAb experiments [47]. While it is thought that these cells represent a key pathogenic element of MS lesions, neither the antigenic specificity of CD8+ T cells nor their role has been elucidated. 
Figure 5: $\mathrm{T}$ cells become activated in the periphery and cross the blood-brain barrier

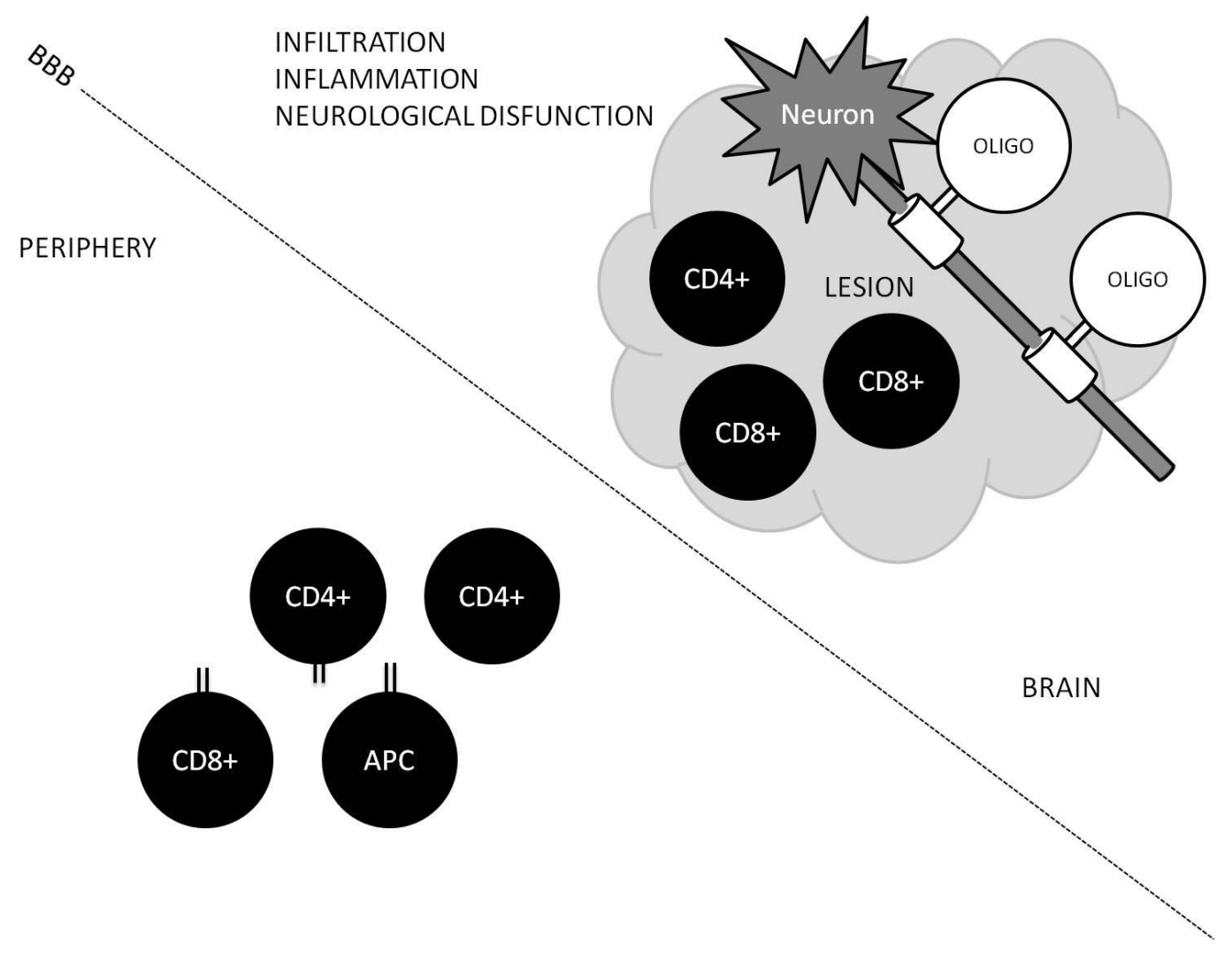

While CD8+ T cells are outnumbered by CD4+ T cells in the periphery, CD8+ T cells are predominant in MS lesions of the CNS 


\section{MULTIPLE SCLEROSIS}

MS is characterized by inflammation, demyelination, and degeneration within the CNS [107], and is the most common debilitating neurological disease of young people [108]. Although, the exact etiology remains unknown, autoimmunity and immune dysfunction is thought to play a role. The result is dysfunctional myelin, the lipid-rich insulation of neural tissue. The myelin of the brain and spinal cord are affected, leading to a variety of neurological defects (Figure 6). Young adults, predominantly Caucasian women, between their third and fifth decades of life are most commonly affected. Approximately one in every thousand persons in the United States (US) is diagnosed with MS. MS is associated with significant impairment caused by neurologic defects in motor, sensory, cognitive, and autonomic tracts. The clinical presentation corresponds to temporally and spatially separated lesions of the CNS. 
Figure 6: Demyelination syndrome subtypes are classified by clinical progression of neurological disability

CLINICALLY ISOLATED SYNDROME

RELAPSING-REMITTING MULTIPLE SCLEROSIS

SECONARDY PROGRESSIVE
MULTIPLE SCLEROSIS

SECONARDY PROGRESSIVE
MULTIPLE SCLEROSIS
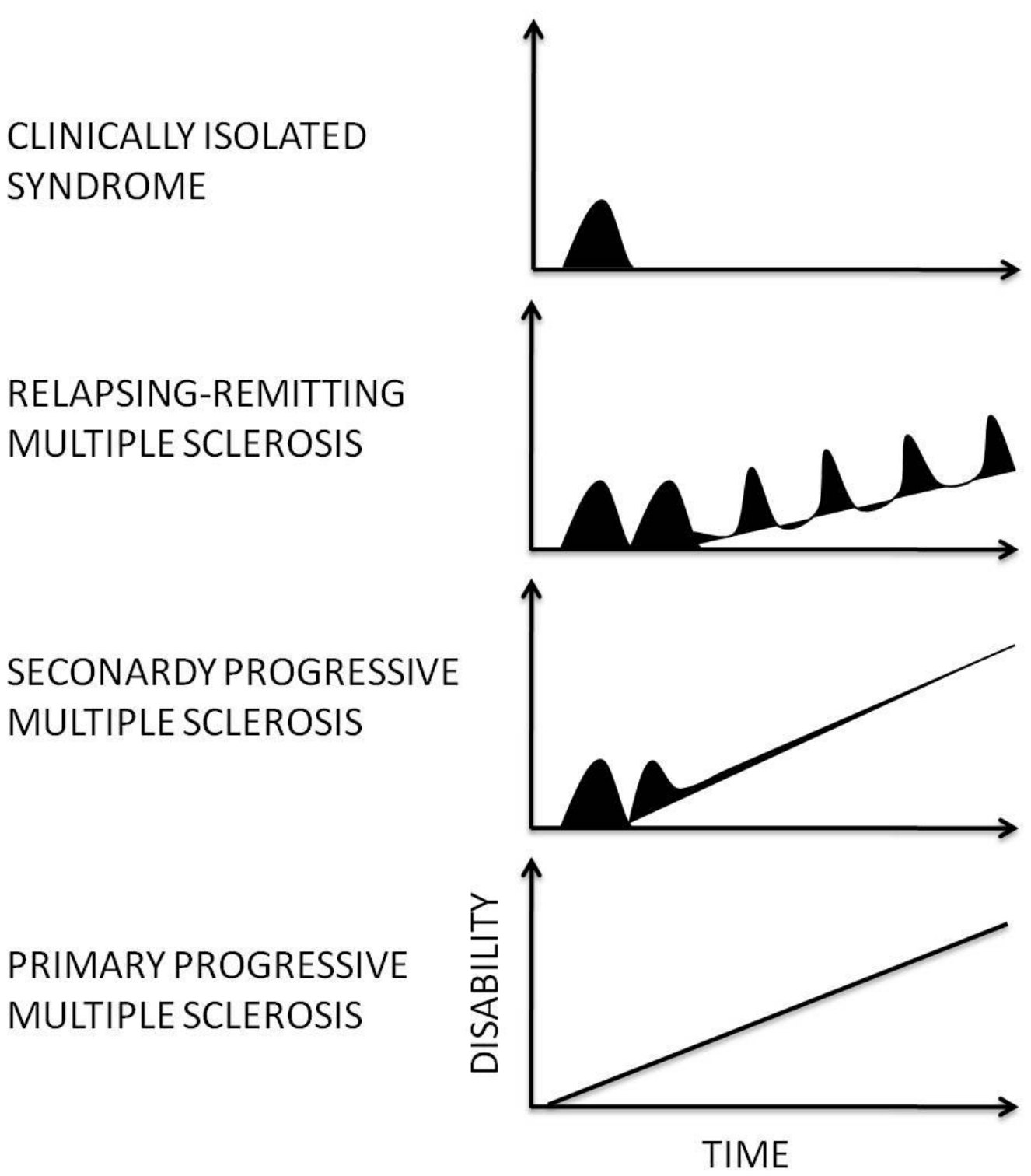

Symptoms may include, but are not limited to fatigue, optic neuritis, weakness, pain, cognitive impairment, depression, spasms, ataxia, paraesthesias, and incontinence. 


\section{BASIS OF PATHOLOGY}

Lesions or plaques of the CNS are a critical functional requirement for the pathologic diagnosis of MS [109]. White matter, a specialized neurological tissue especially rich in myelin, is primarily affected. Post-mortem and imaging studies reveal infiltration by mononuclear cells into mostly the white matter, but also grey matter of the CNS [110]. Luxol fast blue, histological sections from tissue taken from MS patients (Figure 7), reveal decreased myelination, and hematoxylin and eosin (H\&E) stains demonstrate increased cellularity of lesions as compared to healthy subjects [111]. Plaques exhibit sharply demarcated margins of inflammation and demyelination [112]. Active demyelination plaques contain lymphocytes and histiocytes concentrated around blood vessels. Grossly, demyelination is multifocal, located throughout the CNS, but concentrated perivascularly and periventricularly. The size and shape of the plaque(s) can be variable and are asymmetrically distributed. Lymphocytes and macrophages must first cross the open, damaged blood-brain barrier (BBB) and attack myelin and myelin-associated antigens. Glial cells, such as the oligodendrocytes and Schwann cells, are destroyed, thereby impeding conduction of neuronal impulses. Even if spared of transection, axons may still function, albeit poorly [113]. Episodic attacks of demyelination are separated by periods of inactivity or remission. Once the level of inflammatory cells has decreased, astrocytes attempt to repair the active lesion and a gliotic process produces scar tissue. Oligodendrocyte progenitor cells are active in re-myelination. Proliferation by astrocytes, also competent APCs, and further lymphocyte infiltration may lead to 
further immune cell-reactivation in the lesion [114]. Usually widespread, multiple lesions often develop distributed over space and time. The optic tracts, cerebrum, brain stem, cerebellum, and spinal cord are frequently affected. End stage effects are atrophy and degeneration of the brain and spinal cord.

\section{Figure 7: Axial luxol-fast blue staining of cervical spinal cord}

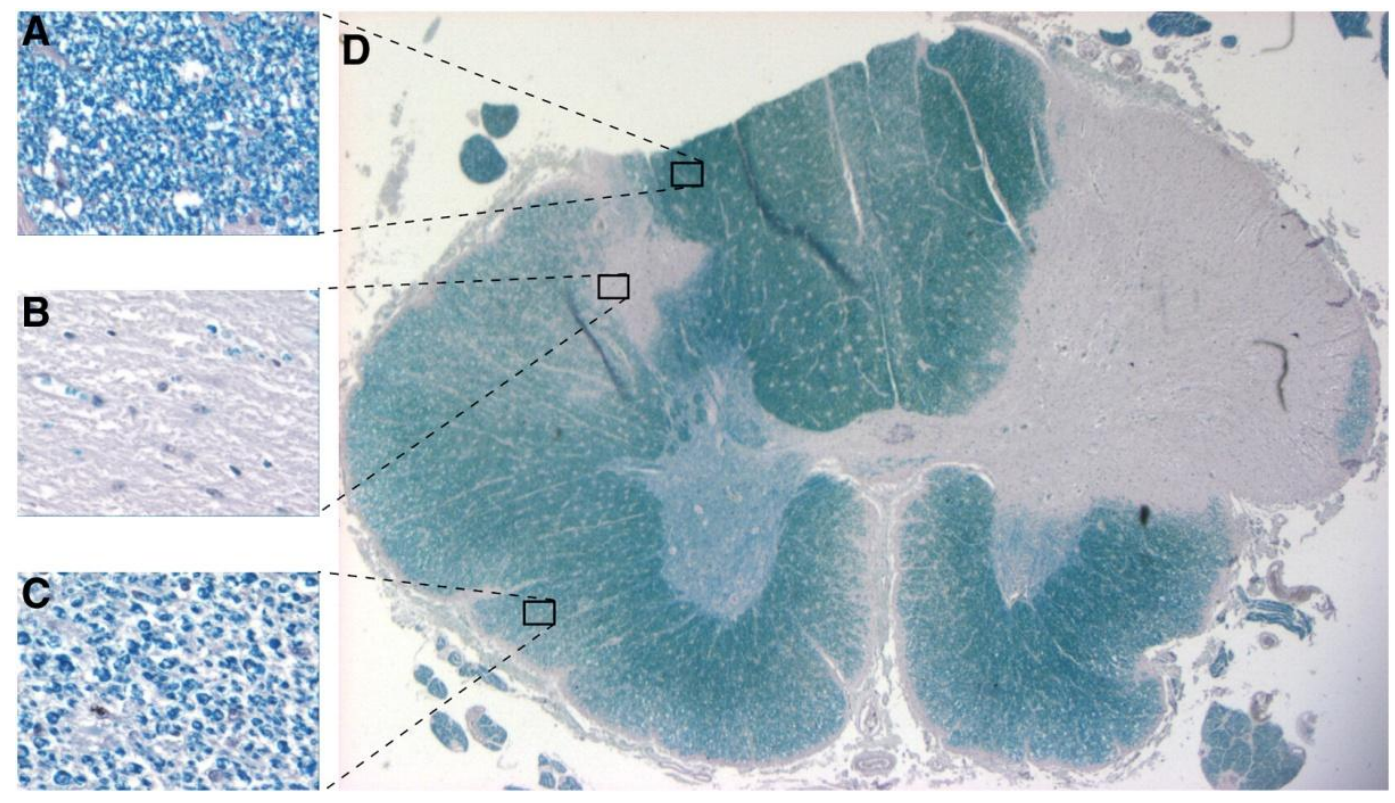

(D) exhibits variable degrees of demyelination in an MS subject: (A) normal myelination, (B) severe demyelination, (C) mild demyelination [115] 
Four distinct subtypes of MS histopathology have been observed [116]. Type one lesions contain mostly $\mathrm{T}$ cells with early preservation of oligodendrocytes. Type two lesions contain $\mathrm{T}$ cells, $\mathrm{IgG}$, and complement components with intact oligodendrocytes. Unlike type one and two type lesions, type three lesions are not perivascular and exhibit marked loss of myelin and oligodendrocytes. Type four lesions are perivascular with marked loss of oligodendrocytes. Any firm relationship among the subtypes may be tentative. Clinical subtypes lack strong correlation to pathological subtypes. Individual patient lesions are heterogeneously composed of various subtypes. The dynamics and evolution among subtypes of the MS lesion are still unclear.

\section{CLINICAL PRESENTATION}

MS affects mostly young, Caucasian, adult women [117]. Based on the location and frequency of lesions, neurological dysfunction varies widely among MS patients. The most common chief medical complaints are spontaneous visual impairment in one eye, fatigue, heat sensitivity, spasticity, and urinary sphincter dysfunction. However, nearly all MS patients exhibit one of two major forms of MS (Figure 6). The relapsing-remitting form (RRMS) is most common, making up approximately eighty-five to ninety percent. RRMS is characterized by periods of remission that are punctuated by acute exacerbations, which remit with limited accumulation of disability. The acute exacerbation symptoms can be caused by new lesions in new areas of the CNS or reoccurring inflammation in pre-existing lesions. Over two to four decades, the majority of RRMS patients convert to a malignant disease course, known as secondary or chronic progressive 
MS (SPMS) [118]. SPMS patients suffer from a non-remitting accumulation of neurological defects. While RRMS is characterized by frequent new lesions, SPMS, in contrast, exhibits chronic lesions with disseminating degeneration along neural tracts away from the lesion with increasingly widespread atrophy and gliosis. Cumulative axonal degeneration is thought to cause clinical decline in SPMS. Approximately one in three is wheel-chair bound, one in two requires medical assistive devices, and two in three are unable to work due to disability. Men and African Americans are at higher risk of progressive disease. The processes that lead away from inflammatory processes to develop degeneration of SPMS are poorly understood.

Other patients present at the onset with accumulating disability with unremitting new lesions. Known as primary progressive MS (PPMS), this group of patients is so different in clinical nature from RRMS, it has led to speculation that it may be better considered a distinct disorder [119]. Relative to RRMS, progressive MS is understudied and poorly understood.

Heat or infection may often slow or block transmission of neuronal impulse through a pre-existing, dormant lesion. Clinically, this manifests as a worsening of previously observed symptoms, which can be difficult to differentiate from a new lesion. The diagnostic laboratory provides the physician with some tools to address these concerns.

\section{LABORATORY DIAGNOSTICS}

A major class of immunoglobulins (Ig) and a major component of myelin, myelin basic protein (MBP), are found at increased levels in the cerebrospinal 
fluid (CSF) during acute exacerbations of MS [120]. CSF oligoclonal bands of Ig are useful for detecting the immune processes that underlie MS. Magnetic resonance imaging (MRI) of the CNS is useful for detecting white matter lesions of MS [121, 122].

Using Koch's postulates, many etiologic agents have been ruled out. MS is neither contagious, nor monophasic. No conclusive evidence points to a single virus, bacteria, spirochete, simple genetic process, dietary deficiency, coagulopathy, poison, or vascular anomaly to cause MS. To date, no single autoimmune or other marker exists to simply differentiate the clinical presentation of MS from its many mimics. Thus, MS requires an exhaustive effort on the part of physicians to exclude other diseases of myelin, including vascular, degenerative, infectious, inflammatory, oncologic, structural, or other selflimiting etiologies. If no better explanation exists for at least two demyelinating lesions in different areas or times within the CNS, a diagnosis of MS can be made. The differential diagnosis of MS challenges even the most skilled teams of neurologists. In order to determine lesion dissemination in space, MRI provides definitive data needed for the diagnosis of MS [123].

\section{FAMILY HISTORY}

Genetic factors are significant in MS [124]. First-degree relatives of MS patients are twenty to fifty times more likely than the general population to also develop MS. Certain alleles of human leukocyte antigen (HLA), also known as major histocompatibility complex (MHC), class II represent the strongest genetic risk factor for MS but are not a surrogate for the previously mentioned criteria for 
the diagnosis of MS. Implicated in the pathogenesis of MS, HLA class II is required for presentation of antigen to CD4+ $\mathrm{T}$ cells. HLA-DR and -DQ loci account for up to sixty percent of genetic risk for MS. Caucasians, Japanese, and many others carrying the DRB1*1501, DRB5*0101, DQA $1 * 0102$, and DQB1*0602 alleles are at higher risk of developing MS (odds ratios in the range of 2-6). Alleles of HLA class I also predict clinical outcome. Implicated in the modulation of MS, HLA class I is required for presentation of antigen to CD8+ T cells [125]. $\mathrm{A} * 0301, \mathrm{~B} * 07$, and $\mathrm{B} * 12$ alleles are associated with a poor outcome. Alleles $\mathrm{A} * 02, \mathrm{~B} * 44$, and $\mathrm{C} * 05$ are protective, with odds ratios in the range of

0.45-0.81 $\left(\mathrm{p}<10^{-3}\right)$. CD8+ $\mathrm{T}$ cell lines from MS patients with progressive disease were first reported as having defective ability to suppress during a time when "suppressor" cells were a rather poorly accepted phenomenon [126]. Several genomes-wide single nucleotide polymorphism (SNP) analyses have also implicated other molecules of immune regulation, including the interleukin 2 receptor (IL2R $\alpha$ ), also known as (CD25), and IL7R $\alpha$, also known as CD127 [127]. The mechanisms underlying the associated risk inherent to certain alleles are unknown.

\section{ENVIRONMENTAL FACTORS}

MS is distributed geographically in mostly developed and westernized countries [128]. Monozygotic twins are concordant in only approximately twenty-five percent of cases, suggesting that environmental effects are significant as well. Natives of higher latitudes, such as Canada, northern Europe, and northern US (Figure 8), carry an increased risk of developing MS. This elevated 
risk persists even in individuals who migrate toward lower latitudes after childhood. Based on these geographical correlations, respiratory illnesses and vitamin D deficiency have been implicated, but not definitive. A continued search for a viral cause of MS endures, including human herpes virus 6 (HHV-6), Epstein-Barr virus (EBV), and endogenous multiple sclerosis-associated retrovirus (MSRV).

Figure 8: The prevalence of MS is increased at higher latitudes

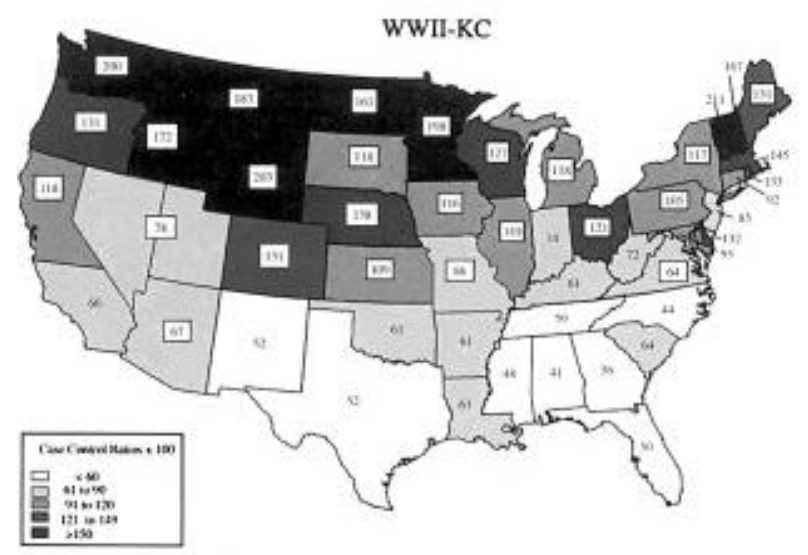

Adjusted case-control ratios $(\times 100)$ were calculated per state for white male veterans of World War II-Korean Conflict (WWII-KC) for MS at entry into active duty [129] 
Other demyelinating syndromes that exhibit similarity to MS are sometimes observed after flu-like illnesses or the administration of experimental vaccines. These demyelinating processes are not a direct result of the pathogen, but rather, are the result of the immune activation. The host immune response is erroneously directed toward self myelin antigens as a result of priming by pathogen antigen, which are similar to self antigens, a microbiologic phenomenon known as molecular mimicry. Paradoxically, vaccination efforts meaning to protect against a viral illness led to a discovery about MS [130, 131]. People and animals vaccinated for rabies and smallpox sometimes develop an ADEM-like syndrome. Both vaccines were initially prepared experimentally using neural tissue. In 1935, studies of rabies vaccine-induced demyelinating disease led many to think for the first time that MS was immune-related. Sterile rabbit-brain emulsions, given intramuscularly without any transmissible agent to macaques, lead to myelin destruction [132]. This demyelinating disease, experimental encephalomyelitis, and experimental autoimmune type one diabetes mellitus (T1DM) are cornerstones of the immune processes thought to be underlying MS [133]. However, a monophasic demyelinating illness, acute disseminated encephalomyelitis (ADEM) is now thought to more directly resemble the current animal models of MS [134] [135]. Classic EAE is monophasic, affects the spinal cord, and displays mild disability that is chronic. Atypical EAE, as well, is monophasic affects the brain. The SJL mouse strain immunized with the epitope of proteolipid protein (PLP) amino acids 139-151, however, exhibits a relapsingremitting form, as a result of epitope spreading [136] [137]. Nonetheless, much 
of what is known about the immunologic processes that underlie MS derives from work in murine EAE, wherein $\mathrm{T}$ cells are major mediators of the acute phase of early disease $[138,139]$. However, it is clear that MS is far more complex than EAE, in that insidious, degenerative processes also contribute to MS disability. EAE is monophasic, while MS is relapsing-remitting. In particular, research toward the immunology of MS, the use of MRI, and the development of new therapies have provided value able insights into MS pathology.

\section{EXPERIMENTAL AUTOIMMUNE ENCEPHALOMYELITIS}

Induced by immunization with myelin-associated antigens, EAE exhibits a variety of neurological defects similar to MS, but predominately an ascending paralysis [139]. Adoptively transferred CD4+ T cells reactive to various CNS antigens are also sufficient for induction of symptoms [138]. EAE is thought to be a Th1-mediated autoimmune disease. However, several studies have suggested pathogenic as well as regulatory involvement of CD8+ $\mathrm{T}$ cell responses. The effect of CD8+ T cells in EAE is largely strain-, vector-, and antigen-specific [140] [141]. The most successful methods for demonstrating the encephalitic potential of CD8+ T cells utilize recombinant virus constructs to express myelin protein [142] [96]. Histological sections of the CNS plaques in EAE show perivascular, mononuclear infiltrates and demyelination, suggestive of immunemediated pathology similar to RRMS and monophasic ADEM.

\section{KNOCKOUT AND MONOCLONAL STUDIES}

EAE performed in a variety of genetic knockout or antibody-treated mice reveals a detailed window into the important players of the disease [143]. 
Compared to wild-type mice, a number of knockout mice, mice treated systemically with monoclonal antibodies or biologic factors display earlier induction and heightened peak disease, underscoring the importance of the following in the prevention, down-regulation, and recovery of EAE: cytokine ciliary neurotrophic factor (CNTF) [144], inducible nitric oxide synthase (iNOS) [145], perforin [146], interferon gamma (IFN $\gamma$ ) [147], IFN $\gamma$ receptor (IFN $\gamma \mathrm{R})$, tumor necrosis factor receptor 2 (TNFRII) [148], interferon- $\alpha / \beta$ receptor (IFNAR) [149], interleukin 10 (IL10) [150], IL15 [151, 152], IL27 receptor (IL27R) [153], CD19 [154], CD8 [155, 156], $\beta 2$ microglobulin [157], HLA-E [158], CD1 [159], cytotoxic T-lymphocyte antigen 4 (CTLA4) [160, 161], programmed death 1 (PD1) [162], CD25 [9], STAT1 [163], and STAT6 [164]. These studies suggest that these markers, transcription factors, and cell types play a regulatory or protective role, and their absence in the knockout is marked by a severe EAE phenotype compared to wild-type.

In contrast, a number of knockout mice, mice treated with monoclonal antibodies and biologic factors display delayed induction, reduced peak disease, or a complete resistance to EAE induction, underscoring the impotance of the following in the initiation, maintenance, and exacerbation of EAE: Fas, Fas ligand (FasL) [165], IL1 [166], IL2 [167], IL4R [150], IL6 [168, 169], IL7 [170], p40p19 subunit double knockout of IL12 and IL23 [171], IL17 [172, 173], p40 subunit of IL23 [174], recombinase-activating genes (RAG) [175], CD4 [155], HLA class II [176], CD28 [177-179], Tbet [180], ROR $\gamma t$ [181], IRF1 [182], STAT4 [164], and Myd88 [183]. This suggests that these players are 
inflammatory and pathogenic in EAE, and that their absence in the knockout exhibits a mild EAE or complete lack thereof compared to wild-type. This information paints in broad strokes the major aspects of the innate and adaptive immune system necessary in order to swell delayed-type hypersensitivity, or cellmediated immunity, and the means necessary to contract, slow, and a reverse that immune response. EAE studies have elucidated the fundamentals of type IV hypersensitivity not only in the context of MS, but also in T1DM, Hashimoto's thyroiditis, rheumatoid arthritis, and contact dermatitis in response to poison ivy or the first immunologic metric, discovered in 1896, the tuberculin (PPD) skin test for exposure to mycobacterium tuberculosis.

The immunologic study of MS through EAE has illustrated a broad theme of critically important $\mathrm{T}$ cell responses that also typify the nature of GuillainBarré syndrome, celiac disease, tuberculosis, graft-versus-host disease, and solid organ transplant rejection. This underscores the importance of CD8+ T cell biology and its application to a variety of clinical scenarios.

\section{REGULATORY CD8+ T CELLS IN EAE AND ANIMALS STUDIES}

A novel population of Qa-1-restricted CD8+ T cells were described with suppressive ability toward CD4+ T cells in EAE [184]. Murine Qa-1 is known as HLA-E in humans. Mice were immunized or not with 9- or 10-mer peptides derived from the TCR V $\beta 8$ CDR2 chain and then all mice were immunized one week later with MBP for the induction of EAE. Mice vaccinated with the CDR2 region showed less paralytic disease and enjoyed a quicker recovery. CD8deficient mice were given the same treatment and lacked any regulation of EAE. 
TCR $\alpha \beta+C D 8 \alpha \alpha+\mathrm{T}$ cells lines were generated using the V $\beta 8$ peptides. The clones were negative for CD62L and expressed high levels of CD28 and the NKG2D homodimer and CD94, which pairs with NKG2A, B, C, and E as heterodimers. CD8 $\alpha \alpha+$ clones secreted IFN $\gamma$ and TNF $\alpha$ and were targeted to kill only $\mathrm{V} \beta 8+\mathrm{CD} 4+\mathrm{T}$ cells. The addition of the single 9-aa peptide (AMAPRTLLL) derived from the leader sequence of many MHC class Ia proteins and referred to as the Qa-1 determinant modifier (QDM) in mice, blocked the proliferative response by a representative CD8+ Treg clone. Qa-1 expression by MBP-reactive CD4 $+\mathrm{T}$ cells was required for killing by $\mathrm{CD} 8 \alpha \alpha+\mathrm{T}$ cells. Adoptive transfer of CD8 $\alpha \alpha+\mathrm{T}$ cells into EAE mice decreased paralytic disease. For the first time, a novel CD8 $\alpha \alpha+\mathrm{T}$ cell population was described with regulatory activity in EAE. The role of HLA-E within other subsets of CD8+ $\mathrm{T}$ cells remained poorly understood.

Studies by Chen and colleagues have elucidated the role of $\mathrm{T}$ cell activation in regulation by other $\mathrm{T}$ cells. Qa-1-restricted CD8+ T cells targeted HSP60sp peptide bound to Qa-1 [185]. HSP60 binding to Qa-1 protects target cells from killing-mediated by QDM and NK cells. HSP60 and QDM compete for binding to Qa-1. The relative ratio of each complex in activated $\mathrm{T}$ cells may provide the biological basis of regulation. $\mathrm{CD} 8+\mathrm{T}$ cells selectively downregulated intermediately activated CD4+ T cells by recognizing HSP60-Qa-1 complex. Intermediately-avidity $\mathrm{T}$ cells expressed the highest ratio of HSP60/QDM complexes of peptide-Qa-1. Vaccination with HSP60-loaded DCs protected mice from paralysis of EAE and increased Qa-1-restricted CD8+ T cells 
specific for HSP60-Qa-1. T cell activation may role an important role in antigenprocessing and expression of HLA class I. The mechanism of relative expression of HSP60/QDM complexes of peptide-Qa-1 remains poorly understood. The avidity model of Qa-1-mediated regulation has important implications based on thymic selection. Self-reactive T cells of high avidity are most likely to undergo programmed cell death in the thymus, thereby enriching the pool of intermediate avidity autoreactive $\mathrm{T}$ cells in the periphery. HSP60-Qa-1 complexes may provide a mechanism of peripheral tolerance.

Recent studies in my lab by York and colleagues have demonstrated the potential immune regulatory role of CNS-reactive CD8+ T cells in EAE [106]. Several induced models of EAE generated CD8 $+\mathrm{T}$ cells with adoptive immune suppressive activity, including $\mathrm{MOG}_{35-55}, \mathrm{PLP}_{139-151}$, and $\mathrm{MBP}_{1-11}$. $\mathrm{MOG}_{35-55^{-}}$ reactive $\mathrm{CD} 8+\mathrm{T}$ cells response were prevalent in active EAE mice. Adoptively transferred into recipients, these cells decreased EAE paralysis compared to OVA-reactive CD8+ $\mathrm{T}$ cells. When $\mathrm{MOG}_{35-55}$-loaded target cells were coadoptively transferred into recipient mice, $\mathrm{MOG}_{35-55}$-reactive $\mathrm{CD} 8+\mathrm{T}$ cells decreased targets in vivo compared to OVA-reactive CD8+ T cells and modulated APC function. While HLA-E may have important roles in the presentation of GA and TCR-derived peptides to CD8+ T cells, the role of HLA-E in regulation of EAE by CNS-reactive CD8+ T cells remains poorly understood.

\section{IMMUNOLOGIC BASIS OF MS}

Initiation of demyelination in EAE requires high-avidity CD4+ T cells that are specific for myelin antigens, such as MBP, myelin oligodendrocyte 
glycoprotein (MOG), proteolipid protein (PLP), myelin-associated glycoprotein (MAG), or others [186][187]. This is not clearly established in humans. Although, autoreactive T cells alone are not sufficient to cause disease in healthy individuals [188]. Autoantigen-specific T cells may function improperly by possessing dual specificity due to the expression of two different beta chains of the TCR [189] [190, 191]. Although, antigen specificity of T cell populations in patients with MS is largely similar to that of healthy individuals [192]. While some myelin epitopes are thought to be more discriminatory in predicting those individuals who are at high risk of MS, no statistically significant differences exist between healthy and MS in the quantity of any bulk T cell population [193] or frequency of certain antigen-specific T cells [194]. In addition to the preexisting autoantigen-specific TCR repertoire, an unknown, inciting environmental insult is thought to trigger a slightly altered differentiation, migration, and expansion of certain T cell populations in acute MS [195] [196]. Pleocytosis and oligoclonal bands are present in a fraction, but not all, of acute MS patients [197]. T cells have seen their antigen in vivo and become activated [198]. T cells cloned directly from MS brain lesions revealed that a significant portion of $\mathrm{T}$ cells infiltrating lesions are not specific to putative MS-related encephalitic epitopes and that CNS infiltration may be the result of by-stander activation [199]. Clones derived from the brain, spinal fluid, and blood of MS patients did not proliferate in response to MBP or PLP, in constrast, to prevalent MBP-specific CD4+ T cells found from post-infectious encephalitis brain tissue. CNS resident autoreactive T cells are not exclustive to MS. 
IFN $\gamma$-producing Th1 and IL-17-producing helper type 17 (Th17) CD4+ T cells are required to infiltrate and propagate CNS lesions. How antigen is initially presented to pathogenic CD4+ T cells is unclear, but probably involves transient, systemic inflammation, leading to acute phase reactants (IL-1, IL-6, and TNFa), which opens the BBB, leading to increase immune cell trafficking into the CNS and spinal fluid compartment [200]. Neuroantigen-specific T cells, as well as other acitivated $\mathrm{T}$ cell populations, cross the blood-brain barrier (BBB). Highavidity CD4+ $\mathrm{T}$ cells encounter their cognate/specific antigen. Local inflammation leaks myelin antigen into the periphery. The antigen is picked up by macrophages, dendritic cells, B cells, or microglia. The context of the antigen presentation dictates the activation status and reaction of further autoantigenspecific CD4+ $\mathrm{T}$ cells encountering their antigen. Having overcome anergy through co-stimulation by APC, activated myelin-reactive CD4+ T cells expand in number probably in a local cervical lymph node. The differentiation of naïve CD4+ T cells into Th1 and Th17 T cells and migration into the CNS allows restimulation after the antigen priming by APC. Activated T cells slow to a roll on the cerebrovascular endothelium through binding selections and adhesions molecules, such as VCAM. Adhering T cells express MMP that degrade collagen and fibronection, permitting trafficking across the damaged BBB. MRI studies visualize that the $\mathrm{BBB}$ is disrupted typically for a month before resolution. Most often, BBB disruption goes unnoticed during the appearance of "silent lesions" [201]. During this time, Th1 and Th17 cells release cytokines and chemokines in the CNS. Tumor necrosis factor alpha (TNF $\alpha)$ and IFN $\gamma$ production leads to 
further T cell, macrophage, microglia, and other immune cell extravasation to the CNS. Activated IFN $\gamma, \mathrm{TNF} \alpha$, and IL-1-producing macrophages, autoantibodyproducing B cells, complement, and free radical release contribute to demyelination. Immune cell to cell contact-mediated damage plays a major role in the dysfunction of myelin. Myelin breaks down with variability in acute lesions. Oligodendrocytes and neuronal axons degenerate from apoptotic or necrotic processes.

\section{CLONALITY OF T CELLS IN MS}

MS is thought to involve an auto-immune responses directed against CNS myelin antigens. Th1 cells are assumed to be the primary immune culprit leading the autoimmune infiltration into the CNS with an important role for macrophages and IFN $\gamma$. The exact identification of the target antigen has been debated. A ground-breaking study by Babbe and colleagues reported the use of microdissection to study the clonality of T cells in MS lesions [95]. MS lesion tissue sections were stained frozen for CD4 and CD8, clearly identifying T cells. A micromanipulator separated single $\mathrm{T}$ cells from surrounding tissue. As held by the theory of clonal specificity, the feature of the $\mathrm{T}$ cells of highest importance was the TCR. As described previously of clonal specificity, only the descendants of a clone share the same TCR. The TCRs of the microdissected lymphocytes were analyzed. For the first time, the analysis revealed that the infiltrating CD8+ $\mathrm{T}$ cell represented the descendants of only a few less numerous and different CD8+ T cell clones, which came to dominate the lesion infiltrate. The oligoclonal $\mathrm{CD} 8+\mathrm{T}$ cells that came to dominate the MS lesion suggested that CD8+ $\mathrm{T}$ cells 
played a more important role in the disease process than previously appreciated. A different study showed that the same brain-infiltrating CD8+ $\mathrm{T}$ cell clone may persist in the CSF and blood for as long at 5 years [102]. It is still unclear if the oligoclonal persistence of $\mathrm{CD} 8+\mathrm{T}$ cells in the lesion is due to selective recruitment of certain clones or random migration of overly representative clones from the periphery. The way to approach this question would be to isolate cells from the periphery in order compare TCR sequencing analysis to cells form the CNS.

Using peripheral cells, my lab analyzed $\mathrm{T}$ cell proliferation using CFSE. The study focused on the antigen targets of CD4+ and CD8+ T cells that mediate the anti-myelin response using a wide spectrum of epitopes [202]. Crawford and colleagues identified HLA-restricted CNS-specific CD4+ and CD8+ T cell populations in untreated MS patients by measuring proliferative responses to 530 serial overlapping peptides spanning the entire sequence of 9 CNS autoantigens, including MBP, PLP, MOG, and 6 others. They assessed T cells proliferative responses, phenotype, and cytokine secretion from patients from various stages of MS. CNS-specific CD4+ T cells were highly prevalent but not significantly altered among healthy persons and all MS patient groups. MS patients' myelinspecific CD4+ T cells did produce increased IFN $\gamma$ and decreased IL4, IL5, and CCR5 compared to healthy persons' cells. This suggests that MS patients' myelin-specific CD4+ $\mathrm{T}$ cells are not quantitatively significantly different than healthy cells, but more differentiated and antigen-experienced. The study revealed an intriguing pattern from the CD8+ $\mathrm{T}$ cell responses. Among RRMS 
patients, the most common autoreactive $\mathrm{CD} 8+\mathrm{T}$ cell responses were MOBPspecific. MS patients' myelin-specific CD8+ T cells produced increased IFN $\gamma$, IL10, and CXCR3. The functions of CD8+ T cells in the CNS infiltrate remained unclear.

Evidence supported two opposing roles for CD8+ T cells in MS. In the experimental autoimmune encephalomyelitis (EAE) model of MS, using transgenic and wild-type mice, studies have revealed a potential pathogenic and regulatory role. Mice genetically depleted of CD8 T cells showed increased relapses in EAE [156]. Recent studies in EAE in my lab have demonstrated a regulatory role for CNS-specific CD8+ $\mathrm{T}$ cells. Earlier human studies had compared either neuroantigen-specific T cells clones' TCR sequences from predominantly the CNS and/or periphery from healthy subject or MS patients. The comprehensive TCR sequencing studies in MS performed by my lab are reviewed here.

Recently, my lab performed a clonal TCR sequence analyses using peripheral neuroantigen-specific CD4+ and CD8+ $\mathrm{T}$ cells from leukapheresed untreated RRMS patients and healthy controls [203, 204]. Biegler and colleagues then performed a basic local alignment search tool (BLAST) search with the dominant clone sequences among published TCR data. Previous studies have shown limited sensitivity to detect differences between healthy persons and MS patients when using fluorochrome-conjugated antibodies to V $\beta$ segments [205]. Alternatively, my lab analyzed TCR complementary-determining region (CDR3) $\mathrm{V} \beta$ sequences by short-term culture with MBP or PLP, flow-sorting of CFSE low 
populations, and an anchored PCR approach. The anchored approach took advantage of a proprietary SMART switching mechanism at the 5' end of the RNA transcript to avoid the requirement of multiple 5' primers for each variable region segment of the $\mathrm{V} \beta \mathrm{CDR} 3$. Flow cytometric sorting of electronically gated CFSE low cell after stimulation with 530 different overlapping peptides of 9 putative target CNS myelin antigen provided pure populations of myelin-specific CD4 and CD8+ T cells for PCR analysis. Healthy persons showed a more focused colonality of MBP-specific CD8+ T cells compared to untreated MS patients. PLP-specific CD4+ T cells among untreated MS patients showed less focused clonality and increased polyclonal TCR repertoire compared to MBPspecific responses. Upon treatment of MS patients with glatiramer acetate, the CD8+ T cell repertoire evolved into a focused, oligoclonal GA-specific V $\beta$ usage. GA-specific CD4+ T cell responses remained less focused, polyclonal in nature and continually changing after months of GA therapy. Published TCR sequences were then interrogated using a BLAST search. Sequences were compared to that of published sequences to better understand the dynamics of CNS-specific CD4+ and CD8+ T cells. BLASTing MS patient sequences in an open database for similarity revealed a large number of MBP- and PLP-specific CD8+ T cell TCR matches of four or greater amino acids in the CDR3 V-NDN-J hypervariable region. Four matches were found among sequences from micro-dissected CNSinfiltrating CD8 $+\mathrm{T}$ cells in MS lesions [95]. One MBP-specific CD8+ T cell clone, representing $12.5 \%$ of total clones, isolated from an MS patient in the 2011 Biegler study shared the same LAGQG CDR3 V $\beta$ sequence as one CD8+ T cells 
microdissected from an active lesion in the 2000 Babbe study. A similar sequence was also found in the peripheral blood of the 2004 Skulina study which persists for years. An important role for auto-reactive CD8+ T cell clones is highlighted by these studies. Their exact function can not be deduced by their CDR3 V $\beta$ usage, and it remains unclear what suppressor potential these particular CD8+ clones possess. A TCR transgenic mouse using the sequence of interest may reveal its encephalitic or regulatory potential. Biegler and colleages BLAST searches have also highlighted similarities between MS TCR V $\beta$ CDR3 sequences and sequences from non-MS patient controls, including arthritis [206], calcified aortic stenosis [207], and chronic encephalitis of Rasmussen [208]. Commonly in healthy persons, focused oligoclonality of CD8+ T cell TCR V $\beta$ CDR3 usage is higher than that among CD4+ T cells. The degree of clonal distribution, through GA therapy or other antigen exposure, may be an indirect and inverse indicator of regulatory $\mathrm{CD} 8+\mathrm{T}$ cell function. Reoccurring conserved CD8+ T cell clones may also target pathogenic $\mathrm{T}$ cells or antigen-presenting cells. Functional assays are the preferred approach to tackling these and other questions.

\section{TREATMENT}

Numerous drugs target the immune processes of MS, which are generally divided into those that are immunomodulatory versus immunosuppressive [120]. Considered here are disease-modifying agents (DMA), rather than therapeutics reserved for the treatment of symptoms, such as spasticity, depression, etc. Prevention of relapse is a major objective of DMA [209]. Immunosuppressive agents are generally only indicated for acute flairs or patients whose disease is 
resistant to DMA. Corticosteroids (steroids), type-one interferon beta (IFN $\beta$ ) (betaseron/Avonex ${ }^{\circledR}$ and extavia/Rebif $\left.\AA\right)$, glatiramer acetate (GA/Copaxone $\left.{ }^{\circledR}\right)$, and natalizumab (Tysabri®) are approved for use in RRMS and progressive disease by the food and drug administration (FDA), while mitoxantrone (Novantrone ${ }^{\circledR}$ ) is approved for use in progressive MS. IFN $\beta$ and GA both reduce relapse rate by thirty percent [210-218]. Natalizumab and mitoxantrone both reduce relapse rate by seventy percent $[219,220]$. Several other generally immunosuppressive agents, while not FDA-approved, have efficacy in MS, including azathioprine, methotrexate, rituximab, anti-CD52 (CAMPATH), mycophenolate mofetil, cladribine, and cyclophosphamide. Heroic efforts to perturb circulating Ig or immune status are occasionally reported with limited efficacy, including intravenous Ig (IVIG), leukophoresis [194], plasmaphoresis, and even bone marrow transplant as salvage therapy of the most extreme cases of terminally progressive MS [221]. While each therapeutic measure carries its own unique set of risks and benefits, the underlying processes and mechanism for many are still under investigation.

\section{CORTICOSTEROIDS}

The mainstay treatment of acute exacerbations of MS is steroids or adrenocorticotropic hormone [222]. Steroids are functionally diverse, nonspecifically anti-inflammatory and immunosuppressive through binding a cytosolic steroid receptor (Gold, Buttgereit et al. 2001). Leukocyte trafficking and matrix metalloproteinase activity in the CSF of MS patients is also decreased by steroids (Rosenberg, Dencoff et al. 1996). Levels of CD4+, CD8+, CD14+ cell 
populations, T-bet expression and signal transducers and activators of transcription protein 1 (STAT1) phosphorylation are all down-regulated by steroid therapy in MS (Frisullo, Nociti et al. 2007). CD8+ T cells have been shown to be less transcriptionally affected by steroid therapy as compared to CD4+ T cells (Li, Leung et al. 2007). Studies in EAE have shown that steroid therapy reduces the frequency of neuroantigen-specific $\mathrm{T}$ cells and spinal cord infiltration (McCombe, Nickson et al. 1996). Steroids have limited potential as prophylaxis. While steroids are known to accelerate recovery in MS acute exacerbations and mitigate subsequent neurological deficits, the exact effect of steroids on neuroantigen-specific regulatory $\mathrm{T}$ cells is unclear.

\section{GLATIRAMER ACETATE}

GA (copolymer-1, Copaxone®) is a synthetic, random length, variable sequence peptide composed of the amino acids alanine, lysine, glutamic acid and tyrosine (4.2:3.4:2.1:1 approximate ratio), designed at the Weizmann Institute in 1971 to mimic myelin basic protein (MBP) through EAE studies [223-228]. Peptides vary from 40 to 100 amino acids in length with an average molecular weight of five to nine kilo-Daltons [229]. Rather than having the intended encephalitic potential of MBP, GA surprisingly protected against encephalitis. GA is now the second most prescribed drug for MS with approximately one in eight MS patients in the US having a long-term prescription for GA [230]. Several mechanisms of action have been proposed for GA [231]. Some reports have suggested that it causes a Th1 to Th2 shift [232] or modulated CD4+ T cell apoptotic process [233]. Highly immunogenic with respect to $\mathrm{T}$ cells, GA alters the $\mathrm{T}$ cell receptor (TCR) 
repertoire and increases suppressor/regulatory $\mathrm{T}$ cell (Treg) function. GA induces anti-inflammatory type 2 monocytes and increases forkhead box P3 (FOXP3) protein expression by CD4+ T cells. The effect of GA likely also involves dendritic cells, which are activated and traffic to lymph nodes [234]. Highlyinducible GA-specific CD4+ and CD8+ T cell responses are present in healthy persons, but are deficient in untreated MS patients. GA-specific $\mathrm{T}$ cell populations are expanded while myelin-specific $\mathrm{T}$ cells are perturbed in a manner still not completely understood. GA may act as an altered peptide ligand, antagonizing MBP 82-100 neuroantigen-specific T cells [235-237]. Over months of therapy, GA induces an extraordinary class of regulatory CD8+ T cells, possessing cytotoxic ability toward CD4+ T cells that display GA in the context of HLA-class I and non-classical HLA-E. This suggests that GA utilizes the cross-presentation pathway of exogenous antigen into HLA class I. Analysis of TCR rearrangements selected for by GA therapy has revealed that GA induces a distinctly focused, oligoclonal CD8+ $\mathrm{T}$ cell response in comparison to CD4+ $\mathrm{T}$ cells. This argues against the proposed theory of a Th1 differentiation shift toward helper CD4+ T cells type 2 (Th2) [238]. The therapeutically-induced GAspecific CD8+ T cells express both effector and regulatory cytokines, suggesting that cytotoxicity and suppression are related, contrary to prior thought [239]. In the perforin, CD8, or indoleamine-pyrrole 2,3-dioxygenase (IDO) knockout mice, GA treatment has an exacerbating effect on EAE. This suggests that GA activates antigen-presenting cells (APC) and that IDO expression by APC is necessary for the induction of perforin-expressing CD8+ Treg. In addition to 
immunomodulation, neurotrophic properties are exhibited by GA, inducing CD4+ $\mathrm{T}$ cell production of bone-marrow derived neurotrophic factor (BDNF). This suggests that neurotrophic effects and may explain how GA-specific T cells protect from optic nerve crush injuries [240]. It is unclear which epitopes of the GA mixture contain the most potent immunomodulatory and neurotrophic effects or what surface and intracellular molecules determine how cells transmit these signals.

\section{BETA INTERFERONS}

Pharmaceutical grade IFN $\beta$ is derived from a naturally-occurring biologic and is the most commonly prescribed drug for MS, with approximately 29 percent of patients in the US having a long-term use prescription. Endogenous IFN $\beta$ production by virally infected cells interferes with viral replication and spread. However, exogenous IFN $\beta$ administration modulates the immune system through induction of numerous proteins through binding of the IFN $\alpha / \beta$ receptor. HLA class I expression is increased on all nucleated cells. Professional APCs are activated, and other innate immune processes are activated. The adaptive immune system is indirectly modulated by the increased efficiency of antigen turnover and presentation to both $\mathrm{CD} 4+$ and $\mathrm{CD} 8+\mathrm{T}$ cells. IFN $\beta$ decreases expression of matrix metalloproteases (MMP), which are necessary for trafficking across the BBB. The effects of IFN $\beta$ on suppressor cells function remain unclear.

\section{NATALIZUMAB}

Natalizumab is a monoclonal antibody targeted toward very late antigen-4 (VLA4), preventing $\mathrm{T}$ cells, $\mathrm{B}$ cells, and macrophages from binding vascular 
adhesion molecule (VCAM) and trafficking into the CNS. Although natalizumab exhibits potent efficacy, anti-VLA4 monoclonal antibodies carry an associated, significant risk of opportunistic infection. A rare, but significant, portion of patients taking natalizumab develop progressive multifocal leukoencephalopthy (PML), caused by JC viral infection of CNS tissue. This briefly led to the removal of the drug from the market in the US. Abrogation of immune cell extravasation by natalizumab far outlives its half-life, significantly decreasing leukocytes generally, specifically CD4+ T cells, CD8+ T cells, CD19+ B cells, and CD138+ plasma cells in CSF from natalizumab-treated patients for up to 6 months after discontinuation of natalizumab [241]. Surveillance of CNS tissue is a necessary function of lymphocytes to prevent opportunistic and infectious pathogens. Thus, there is a pressing need for an equally powerful, but less blunt therapy, targeting specific regulatory and pathogenic immune cells in MS [242].

\section{FINGOLIMOD}

Fingolimod $\quad\left(\right.$ Gilenya $\left.{ }^{\circledR}\right) \quad$ is $\quad$ an $\quad$ orally administered immunomodulatory/immunosuppressive agent, capable of reducing relapses in MS by fifty percent. It sequesters lymphocytes in lymph nodes though binding of sphingosine receptors. Patients who take fingolimod may suffer from increased risk of respiratory infections and cancer. In vitro studies and murine models have suggested that fingolimod may inhibit regulatory $\mathrm{T}$ cell function. It remains unclear if this effect is selectively targeted toward CD4+CD25+ cells or if fingolimod generally inhibits T cell proliferation.

\section{MITOXANTRONE}


Mitoxantrone (MX) is a cytotoxic, generally immunosuppressive agent, used mostly for patients who are poorly responsive to the previously-mentioned immunomodulators. Typical side effects of all chemotherapeautic agents include bone marrow suppression, hair loss, diarrhea, immunodeficiency, nausea, and fatigue. MX, in particular, can cause cardiomyopathy, but is well tolerated in most patients. Some evidence points to an ability of MX to induce regulatory $\mathrm{T}$ cells. However, it remains unclear if regulatory $\mathrm{T}$ cells are selectively resistant to the effect of or induced by MX.

Nearly all approved and non-approved drugs are still under intense investigation. The most important aspect of these agents is that they work, while exactly how they work is worthy of much on-going research. It is important to note that the animal models of MS are to thank for the discovery of many of these therapeutics.

\section{DEFICIENT CENTRAL TOLERANCE IN MS}

The event triggering the cascade of failed tolerance before the encephalitic event of MS is unclear. Most theories develop chronologically from the standpoint of central tolerance, suggesting $\mathrm{T}$ cell selection in the thymus is dysfunctional. Peripheral tolerance must then also fail, setting up a swiss cheese model of several regulatory mechanisms allowing a overzealous immune response to slip through. The first critical aspect is discussed here. Initially, certain HLA class II alleles must give rise to extraordinary autoreactive CD4+ T cells in one or more of several proposed ways. High risk HLA alleles preferentially bind to a set of myelin, self peptides in the periphery. This may explain a predisposed heightened ability of T cells to react to myelin antigen in the CNS. Alternatively, high risk 
HLA alleles preferentially do not bind a set of myelin, self peptides in the thymus during $\mathrm{T}$ cell development. The alternative may explain a deficient negative selection process, allowing myelin-specific $\mathrm{T}$ cells to escape thymic selection into the periphery. The other major explanations focus upon polymorphisms of the cytokines and $\mathrm{T}$ cell receptor (TCR) genes, having effects on TCR affinities, frequency, and differentiation of autoreactive $\mathrm{T}$ cells found within the $\mathrm{T}$ cell repertoire. Beyond the considerable complexity of thymic selection and genetic background, other major determinants to the development to autoimmunity include antigen exposure over a lifetime and several peripheral tolerance mechanisms, including many subsets of Treg. Our understanding of the nature of both highly complex immune and nervous systems remains limited in the context of MS.

\section{DEFICIENT PERIPHERAL TOLERANCE IN MS}

CD4+ Treg have been shown to be deficient in suppressive ability in MS [243, 244]. The role of CD8+ $\mathrm{T}$ cells is less clear with evidence supporting both regulatory and cytotoxic functional profiles $[100,104]$. Evidence suggests that CD4+ and CD8+ Tregs are deficient in untreated MS and required for therapeutic induction [245] [104, 246]. While it is evident that naturally-occurring GAspecific CD8 $+\mathrm{T}$ cells can be therapeutically expanded and confer a protective effect in MS [104], CNS-specific CD8+ T cells remain somewhat enigmatic. How CNS-specific CD8+ $\mathrm{T}$ cells contribute to regulatory activity has yet to be clearly characterized in MS. Functional studies point to the class of C-type lectinlike MHC class Ib-specific inhibitory NK cell receptors expressed by activated 
CD4+ T cells and other APC; CD8+ Treg up-regulation of NKG2A and CD94 was implicated during exacerbations [67]. Cross-presentation may play an important role in the generation and priming of CNS-specific CD8+ T cells in MS. Exogenously-endocytosed self-antigen or GA by microglia, macrophages, and CD4+ T cells may be cross-presented in the context of HLA class I. CD4+ T cells may present self-antigen through dendritic-cell (DC)-derived exosome trafficking and membrane exchange. $\mathrm{CD} 8+\mathrm{T}$ cells may down-regulate CD4+ T cells after they exchange membranes with APCs, which confers antigen-bearing HLA class I to CD4+ to act as a target cells for regulatory CD8+ T cells [247]. Further functional studies are required to uncover $C D 8+T_{\mathrm{REG}}$ cellular interactions at play in MS. 


\section{CD8+ T CELLS IN HEALTH AND DISEASE}

CD8+ T lymphocytes are critical to understanding viral, malignant, and immunerelated diseases. Cytotoxic potential toward infected and tumor cells is one of CD8 T cells' most well-appreciated attributes. From precursors originating in the bone marrow, CD8+ T cells arise developmentally during thymic selection. In the thymus, thymocytes are selected which are attracted with moderate avidity toward HLA-class I, while nearly all others undergo apoptosis. Central tolerance mechanisms shape the $\mathrm{T}$ cell repertoire and ultimately allows nearly all $\mathrm{T}$ cells to be directed away from self and instead toward non-self (pathogens) and abnormal self (cancer). All nucleated cells express HLA-class I, thereby allowing the surveillance by CD8+ $\mathrm{T}$ cells of nearly every other cell in the body. Through a process of gene rearrangement, each $\mathrm{T}$ cell expresses a unique TCR from every other $\mathrm{T}$ cell. Thymic selection diminishes potentially-autoreactive and poorlyfunctional $\mathrm{T}$ cells by putting their newly rearranged pre-TCR genes to the test. The unique TCR of each $\mathrm{T}$ cell recognizes a specific antigenic peptide of approximately eight to ten amino acids in length in the context of HLA class I. Potentially-autoreactive thymocytes are selected-against through binding strongly to thymic epithelials cells, which ectopically express a variety of tissue specific antigens, transcriptionally regulated by AIRE [248]. The majority of CD8+ T cells, therefore, that escape thymic selection are MHC-class Ia-restricted and possess high avidity for foreign antigens, such as viruses [249, 250]. However, small numbers of low-avidity autoreactive $\mathrm{T}$ cells escape the thymus in healthy humans. 
Once in the periphery, naïve $\mathrm{T}$ cell stimulation and activation is dictated by a primary set of signals. Antigen serves as the first signal, co-stimulation as the second signal, and cytokines as the third signal. T cells respond to various stimuli through mRNA transcription, protein production, and proliferation potential. T cells exhibit extensive plasticity regulated by the context of their activation, largely the status of the APC. Effector T cells may be converted to suppressors (Ts) through APC and paracrine signals. The inappropriate sequence, quantity, or quality of signals leads to $\mathrm{T}$ cell apoptosis or anergy. As CD8+ $\mathrm{T}$ cells are also capable of simply killing abnormal immune cells, they wield an important influence on human disease. Therefore, the nature of low-avidity, self-reactive $\mathrm{CD} 8+\mathrm{T}$ cells remains unclear. Relative to CD4+ $\mathrm{T}$ cells in MS, the role of CD8+ is less clear with evidence supporting both regulatory and cytotoxic functional profiles.

Although much attention focused on $\mathrm{CD} 4+\mathrm{CD} 25+$ inhibition of priming or expansion of $\mathrm{T}$ cell immunity, considerably less has been known about the role of regulatory CD8+ T cells in feedback regulation. Early work by Antel and others in the 1980s presented evidence for activated suppressor cell dysfunction by CD8+ T cells in MS [93, 126, 251] [252]. During acute exacerbations of MS patients, CD8+ $\mathrm{T}$ cells were decreased in the peripherical blood compared to controls. Controlling for numbers, suppressor CD8+ T cells were induced in vitro by stimulation with ConA or anti-CD3. Concanavalin A (ConA) or anti-CD3stimulated CD8+ $\mathrm{T}$ cells were shown to be defective at suppressing fresh autologous ex vivo responder cells during active disease compared to healthy 
persons. On average, MS patient OKT3-induced suppressor cells reduced responder cell proliferation by $30 \%$ while healthy controls reduced proliferation by $70 \%$. CD8+ T cell-enriched cultures showed greater suppression than CD4+ T cell-enriched cultures. The study focused attention for the first time on the defect of regulatory potential by $\mathrm{CD} 8+\mathrm{T}$ cells during untreated progressive MS. While the study pointed to inducible regulatory function of $\mathrm{CD} 8+\mathrm{T}$ cells, the specificity and ex vivo activity of the suppressor cells remained unclear. Other studies suggested that cytolytic activity of CD8+ T cells was selectively preserved while suppressive activity was lost in MS patients. The dominant suppression mediated by CD8+ T cells compared to CD4+ T cells in healthy persons, and its deficit in MS patients highlighted the importance of CD8+ T cells in MS [253]. The subset of CD8 required for suppression and their mechanism remained unknown.

\section{AUTOREACTIVE REGULATORY CD8+ T CELLS}

The ability of healthy human MBP-specific CD8+ T cells to regulate autologous MBP-specific CD4+ $\mathrm{T}$ cells was later reported [254]. A human CD8+ T cell clone blocked proliferation of autologous CD4+ MBP-specific T cells in a manner that required MHC class I. When CD8+ T cells predominated in culture over the CD4+ T cells, cytolytic ability was observed specifically toward MBP-specific CD4+ $\mathrm{T}$ cells, but not herpes simplex virus-specific CD4+ $\mathrm{T}$ cells. It remained unclear what, if any, regulatory role autoreactive CD8+ T cells might have in MS. This led to human studies where MS patients were vaccinated with irradiated MBP-specific $\mathrm{T}$ cells in the hope that regulatory $\mathrm{T}$ cells were induced to deplete circulating MBP-reactive $\mathrm{T}$ cells [255]. After the experimental treatment, 
regulatory CD8+ T cell lines were generated from recipients and showed in vitro suppressive ability and cytotoxicity toward vaccine cells in an HLA-restricted manner. Little clinical effectiveness was observed by Jiang and colleagues and similar studies [256] nor in other human studies involving the use of vaccination with the TCR V $\beta 5.2$ sequence [257]. The principle of interacting autoreactive regulatory $\mathrm{T}$ cell in humans was validated. The effect of other immune-deviating therapies on regulatory CD8+ T cells in MS was still poorly understood.

\section{COPAXONE AND CD8+ T CELLS}

My lab then reported that, a FDA-approved treatment for MS, Copaxone induced CD8+ T cells which kill GA-loaded target cells using MHC class I [104]. The mechanism of Copaxone is unknown. Several proposed mechanism have been debated. The composition and effects of Copaxone are reviewed below. Tennakoon and colleagues showed that naturally-occurring GA-reactive T cells are prevalent in healthy persons while GA-reactive T cells are decreased in MS patients. Anti-CD3-stimulated and GA-reactive CD8+ T cells were functionally suppressive toward CD4+ $\mathrm{T}$ cell proliferation. $\mathrm{CD} 8+\mathrm{T}$ cells mediated suppression through cellular contact. Daily treatment with Copaxone for months by MS patients resulted in an increased suppressive function toward CD4+ T cell proliferation that was HLA class I (A, B, and C)-restricted. GA-specific killing of CD4+ T cells was inhibited by anti-HLA-E antibodies in four of five subjects. Tennakoon and colleagues finally generated antigen-reactive $\mathrm{T}$ cell lines with myelin antigens, Copaxone, and foreign control antigens. Perhaps most interestingly, GA-specific CD8+ T cell lines were capable of suppressing CNS- 
specific CD4+ T cells line proliferation. It remained unclear if autoreactive CD8+ $\mathrm{T}$ cells were deviated by Copaxone therapy or if regulatory CD8+ $\mathrm{T}$ cells originated from a distinct GA-specific lineage. The composition and structure of Copaxone may have allowed it to promiscuously bind to both non-classical HLAE and classical HLA class I, whereas the restriction and antigen targets of suppressive autoreactive CD8+ T cells were still unclear. The role of autoreactive CD8+ T cells in MS remained poorly understood.

\section{AUTOREACTIVE CD8+ T CELLS AND MS}

Debate continued upon the presumed regulatory role of autoreactive CD8+ T cell in MS. For example, two human studies generated similar experimental results and gathered two opposing interpretations. The first study by Zang and colleagues generated MBP-specific CD8+ T cells lines from healthy persons and MS patients [258]. The MBP-specific CD8+ T cells exhibited cytotoxicity toward MBP-loaded autologous B cells or human HLA class I-expressing monkey kidney cell lines. The second study generated CD8+ T cell clones recognizing autologous myelin-reactive CD4+ $\mathrm{T}$ cell clones from the blood and CSF of healthy controls and MS patients during acute exacerbations and remissions [67]. The in vitro expanded CD8+ T cells from MS patients killed autologous myelinspecific CD4+ T cell clones with granules only when target cells were activated and expressing HLA-E. During acute exacerbations of MS, serum levels of IL15 were elevated, and the expression of CD94 and NKG2A by CD8+ T cells was upregulated and therefore decreased cytolytic ability towards CNS-specific CD4+ T cells. In vitro IL15 and IFN $\gamma$ increased CD94/NKG2A expression on CD8+ T 
cells and decreased cytotoxicity towards CD4+ T cells. The authors of the first study by Zang and colleagues dismissed any potential role of human autoreactive CD8+ T cells in suppression of immune responses in MS. In the second study, the Correale and colleagues suggested that cytolytic ability of CD8+ T cells toward other immune cells may act as an important tolerance mechanism. The second study also suggests that cytokine levels an important role on the cytotoxic and regulatory ability of $\mathrm{CD} 8+\mathrm{T}$ cells in MS. Clinical exacerbations may become apparent when physiologic regulatory ability by CNS-specific CD8+ T cells is lost due to increased levels of IL15. The effects of cytokines on regulatory/cytolytic activity of $\mathrm{CD} 8+\mathrm{T}$ cells may differ according to levels of IL15. The controversy surrounding autoreactive CD8+ T cells in MS remains, as exemplified by these two cellular immunology studies. The mechanism by which CD94/NKG2A expression by myelin-specific CD8+ T cells is increased during acute exacerbations of MS remains poorly understood.

\section{BIOLOGY OF HLA CLASS I MOLECULES}

Major histocompatibility complex (MHC) genes, also known as human leukocyte antigen (HLA), have strong associations to autoimmune, infectious, and inflammatory diseases [259]. The murine MHC locus was discovered by George Snell [260]. Jean Dausset discovered HLA on white blood cells [261]. Baruj Benacerraf described the biology of HLA in the immune response [262]. HLA class I molecules present endogenous peptides to $\mathrm{CD} 8+\mathrm{T}$ cells and natural killer (NK) cells for screening of viral infection and malignant transformation [263]. Both classical (Ia) and non-classical (Ib) HLA class I molecules play a critical 
role in immune surveillance and tolerance by acting as ligands for NK cell inhibitory receptors and TCRs of CD8+ T cells. HLA class Ia and Ib molecules are expressed by all nucleated cells and play a critical role in both innate and adaptive immunity. On the cell surface, mature HLA class I contains a heavy chain, $\beta_{2}$ microglobulin light chain, and peptide of eight to ten amino acids. Expression of class I proteins on the cell surface requires endogenous antigenprocessing of peptides within the endoplasmic reticulum (ER). Antigenprocessing from the cytosol into the ER is transporter associated with antigen processing (TAP)-dependent. TAP-deficient animals also express a limited number of peptides that contain a leader signal sequence [264]. Other regions of proteins outside of the leader signal peptide and some leader-derived peptides processed or recycled into the cytosol in a TAP-dependent manner of antigenprocessing and expression in the context of HLA class I [265].

\section{CLASSICAL HLA I}

The highly polymorphic, classical HLA class Ia molecules (A, B, and C) are thought to present a great variety of endogenously synthesized peptides (greater than $10^{5}$ ) to $\mathrm{CD} 8+\mathrm{T}$ cells. $\alpha \beta \mathrm{CD} 8+\mathrm{T}$ cells recognize virus- or self-derived antigens in the context of HLA-Ia. The TCR-peptide-HLA-Ia clonotypic interaction is the foundation of the adaptive immune response, and certain HLA alleles are highly linked to clinical syndromes in humans. The CDRs of the $\alpha$ and $\beta$ TCR chains are positioned in close proximity to the antigen resting within the polymorphic regions of the HLA-Ia molecule peptide-binding groove. The CD8 
molecule augments the interaction through binding of the constant region of HLA-Ia molecules. NON-CLASSICAL HLA

The nearly monomorphic, non-classical HLA-Ib molecules (E, F, and G) have been thought to present only a limited number of peptides [266] [267]. HLAclass Ib pathways have been thought to be evolutionarily older and more conserved than the HLA-Ia pathways. HLA-F has been poorly studied. HLA-G is intermediately polymorphic between strictly $\mathrm{Ia}$ and $\mathrm{Ib}$ molecules [268]. The CD94/NKG2-peptide-HLA-E interaction plays an important role in the innate immune response and discrimination of self/non-self by a small subset of cytotoxic and regulatory $\mathrm{T}$ cells that express CD8. The innately germlineencoded CD94/NKG2 receptors engage the peptide and interface with HLA-E in a similar manner by which hyper-variable TCR binds variable peptide-HLA-class Ia. NK and CD8+ T cells express the CD94/NKG2 hetero-receptor which binds peptide-HLA-E, which decreases cytolytic ability toward the HLA-E expressing cell [269]. HLA-E is expressed by nearly all cells with intact antigen-processing machinery. If a virally infected cell has down-regulated HLA-E expression, NK cell cytolytic ability is increased toward the infected cell [270].

\section{PEPTIDE REPERTOIRE OF HLA-E}

The predominant peptides that fill HLA-E are derived from the signal sequence of classical HLA class Ia. The predominant peptide is known as the Qa-1 determinant modifier in mice (AMAPRTLLL) and B7sp in humans (VMAPRTVLL). All peptides that bind to HLA-E have a nine amino acid 
sequence with methionine second from the $\mathrm{N}$-terminus and leucine at the $\mathrm{C}$ teriminus. Similar to the limited set of peptides presented in HLA-Ia of TAPdeficient animals, HLA-E-presented peptides contain the leader signal sequence of class Ia molecules and similar leader sequences from other proteins [271]. The HLA-E was recently shown to express a leader sequence from heat shock protein (HSP) 60 [272, 273]. The HLA-E-HSP60 complex was no longer recognized by the CD94/NKG2 inhibitory receptor, leading to increased cytolytic ability toward stressed cells. Jiang and colleagues went on to show that HLA-E restricted CD8+ $\mathrm{T}$ cells are involved in the development of autoimmune diabetes type 1 diabetes. HSP60-specific, but not B7sp-specific, HLA-restricted CD8+ T cells were able to suppress the GAD-specific CD4+ T cells and MBP-specific T cells [274]. HSP60 may activate $\mathrm{CD} 8+\mathrm{T}$ cell suppressive activity and $\mathrm{B} 7 \mathrm{sp}$ may inhibit their suppressive activity. The role of CD8+ T cells that are inhibited by B7sp-HLA-E complex is poorly understood.

\section{HLA-E RESTRICTED CD8+ T CELLS}

In addition to an important role in the innate immune system, HLA-E may serve adaptive immunity as well. Recent evidence suggests that the peptide repertoire that is presented by HLA-E is broader than what was once thought [275]. HLA-E may also act as a back-up antigen-presentation pathway to $\alpha \beta \mathrm{CD} 8+\mathrm{T}$ cells in the case of TAP-deficient tumors. Considerable evidence suggests that HLA-E may act as a ligand for the TCR $\alpha \beta$. In mice and human studies, CD8+ T cells proliferate in response to cytomegalovirus, Epstein-Barr virus, Listeria monocytogenes, Mycobacterium tuberculosis, and Salmonella enteric infection in 
a HLA-E restricted manner [276-279]. The mechanism of how foreign antigens replace self peptides during HLA-E antigen-processing is poorly understood. The consequences of innate and adaptive immune cells competing to bind HLA-E are poorly understood. HLA-E may possess dual roles at the interface of innate and adaptive immunity, presenting predominantly conserved leader signal sequences in an inhibiting context and a variable repertoire under conditions of tolerance and infection. The potential regulatory role of HLA-E-restricted CD8+ T cells will be described in the context of EAE and other animal studies. 
POTENIAL FOR CELL BASED THERAPIES IN AUTOIMMUNITY AND TRANSPLANT

While Tregs have been recognized as having an essential role in controlling autoimmunity [280] and prolonging transplant survival [281], individualized ex vivo immune cell-based therapies have yet to become mainstays of therapy for immune-mediated diseases [282]. While ex vivo immune cell-based therapies have reached the clinical trial stage, current protocols have yet to overcome important obstacles [283]. Injected cell populations traffic poorly to lymph nodes, have limited interaction with other immune cells, or are eliminated by the host immune system. The development of dendritic cell and cytotoxic $\mathrm{T}$ lymphocyte-based therapies for bone marrow and skin cancers have shown limited success [284-287]. In contrast, the fixation and transfer of donor cells with the crosslinker agent (ECDI) as therapy has shown promise for antigenspecific tolerization of human solid-organ transplantation [288]. Cancer-specific $\mathrm{CD} 8+\mathrm{T}$ cells co-transferred in conjunction with an autologous hematopoietic transplant confer protection from relapse [289]. Ex vivo-expanded Treg suppress autoimmune T1DM in non-obese diabetic (NOD) mice [17] and GvHD after allogeneic bone marrow transplantation in mice [290]. Antigen-specific therapies, if proven effective, would be a dramatic improvement over non-specific immune modulation. Further mechanistic and functional studies are necessary in order to facilitate wide-spread use of ex vivo immune cell-based therapies.

SUMMARY OF CD8+ SUPPRESSORS 
Much controversy centers upon the role of CD8+ T cells. While most studies of MS and EAE have focused on the role of CD4+ T cells, considerable evidence points to an important role for CD8+ T cells. While it is widely accepted that CD4+ Tregs regulate varying aspects of EAE and MS, their CD8+ counterparts have been left largely understudied and poorly understood. To hasten the advent of novel therapeutic approaches, there is a pressing need for a precise understanding of the immune regulatory and effector roles for CD8+ T cells in MS.

The regulatory $\mathrm{T}$ cell (Treg) field has re-emerged since the decline in the early 1990s, following resurgence in the CD4+ Treg domain. In 1990, it was thought that CD8+ T cells could be divided into two distinct categories. Cytotoxic $\mathrm{T}$ lymphocytes (CTL) were armed for effector function while suppressor CD8+ T cells (Ts) were for modulating responses, distinguished by CD11b- and CD11b+ subsets respectively [291]. Today, the distinction between cytotoxic and regulatory $\mathrm{CD} 8+\mathrm{T}$ cell is less clear than ever. Killing of unwarranted autoreactive cells is a reasonable solution. A major barrier to ex vivo cell-based therapies is that the precise phenotype of CD8+ Treg eludes comprehension. Collectively, several human and mouse studies point to a heterogeneous CD8+ Treg population with suppressive activity in the steady state and upon activation. Evidence for an important role of naturally-occurring CD8+ Treg can be found in several human diseases and experimental disease models: experimental autoimmune encephalomyelitis, MS [67], experimental autoimmune uveitis [61, 292], inflammatory bowel disease and infectious colitis [72, 293], T1DM [68], 
and human immunodeficiency virus (HIV) infection [294]. Several in vitro studies have observed CD8+ Treg suppressive effects imparted on B cells [295], CD4+ T cells and CD8+ T cells [71]. Induction of CD8+ Treg in vivo have been observed in the pathogenesis of HIV [294] and Mycobacteria [90] and during the therapeutic induction of tolerance for the purposes of transplant [61, 296, 297], T1DM [298, 299], and multiple sclerosis [104]. In vitro, CD8+ Treg may be expanded through mitogen or antigen-specific means $[46,300]$. While subsets overlap, several putative phenotypes emerge: CD8+IL2R+, CD8+CD28-, CD8+PD-1+, and other activated CD8+ $\mathrm{T}$ cells by upregulation of several costimulatory and cytotoxic molecular markers. Besides the patent use of HLA class-I, CD8+ suppression occurs through contact dependent- (CD28, CTLA-4-, CD80, PD-1, and CD86) and independent-mechanisms (TNF $\alpha, \operatorname{IFN} \gamma \mathrm{R}, \operatorname{IFN} \gamma$, TGF $\beta$, IL10, CCL4 and IDO). The most well accepted CD8+ T cell regulation occurs by a precisely described mechanism involving HLA-E and the CD94/NKG2A complex [59, 301]. Activated T cells express the non-classical MHC, HLA-E, as a function of $\mathrm{T}$ cell receptor (TCR) avidity to their cognate antigen. CD8+ T cells engage HSP60 peptide-HLA-E via CD94 and selectivelydown regulate $\mathrm{T}$ cells with intermediate TCR affinity for self or foreign peptide. The end result for this peripheral regulatory mechanism is that high TCR affinities for foreign antigens are enriched, while only low affinities are allowed for selfspecific TCR. The importance of this regulatory pathway is further illustrated by its perturbed role in MS pathogenesis. During MS exacerbations, CD8+ T cell clones express significantly higher killer-inhibitory receptor CD94/NKG2A, 
which IL-15 and IFN $\gamma$ further increase, and exhibit reduced cytolytic ability towards MBP- and MOG-specific CD4+ $\mathrm{T}$ cells [67]. The transcriptional regulation, plasticity, and dynamics of $\mathrm{CD} 8+\mathrm{T}_{\mathrm{REG}} \mathrm{S}$ in $\mathrm{MS}$ remain unclear throughout different phases of the disease. The precise role of CNS-specific CD8+ T cells in the pathogenesis/regulation of MS is poorly understood.

\section{SUMMARY OF INTRODUCTION}

Multiple sclerosis (MS) is the most common disabling neurological disease of young people of unknown etiology and is thought to be an immune-mediated disease. Much of what is known about the immunologic processes that underlie MS derives from work in murine experimental autoimmune encephalomyelitis (EAE), wherein $\mathrm{T}$ cells are major mediators of disease. Similarly, MS patients harbor CNS-specific T cell responses and mononuclear CNS infiltrates, and exhibit improvement upon treatment with immunomodulatory drugs. To hasten the advent of novel therapeutic approaches, there is a pressing need for a precise understanding of the immune regulatory and effector roles for T cells in MS.

Previous observations from us and others highlight the importance of CD8+ $\mathrm{T}$ cells in MS. MS patients harbor CNS-specific CD8+ T cells displaying both effector and regulatory properties [202]. CNS lesions in MS patients show oligoclonally expanded CD8+ T cells [294]. However, the roles of CD8+ T cells in the pathogenesis and regulation of MS are unclear. Following a course of glatiramer acetate (GA), untreated MS patients exhibit an expansion of CD8+ T cells possessing regulatory activity, which are lacking before treatment as compared to healthy individuals [104]. 
The regulatory $T$ cell $\left(T_{\text {reg }}\right)$ field has re-emerged since the decline in the early 1990s. While most studies of MS and EAE have focused on the role of CD4+ T cells, considerable evidence points to an important role for CD8+ T cells. While it is widely accepted that CD4+ $\mathrm{T}_{\text {reg }}$ s regulate varying aspects of EAE and MS, their CD8+ counterparts have been left understudied largely due to the ongoing surge of CD4+ $\mathrm{T}_{\text {reg }}$ studies starting in late 1990s. CD4+ and CD8+ regulatory $\mathrm{T}$ cells (Treg) are an important part of the immune system that maintain peripheral tolerance in a healthy immune system and may be used to establish therapeutic tolerance to transplants.

\section{HYPOTHESES}

Based on prior studies and preliminary evidence, we hypothesized that CNSspecific CD8+ T cells play an important immunomodulatory role in MS and mediate the effects of clinically successful therapy. In the steady state and during therapeutic induction, distinct subsets of CD8+ $\mathrm{T}$ cells are proposed to be involved in the down-regulation of pathogenic T cell responses. Specific subsets of CD8+ Treg harbor potent regulatory activity. High CD28+ and low CD62L expression positively correlates with high CD8+ Treg suppressive ability. Autoreactive regulatory $\mathrm{CD} 8+\mathrm{T}$ cells use contact-dependent means of suppression toward CD4+CD25- T cells.

\section{SPECIFIC AIMS}

The biology of CNS-specific CD8+ T cells as well as other CD8+ $\mathrm{T}_{\text {reg }}$ subsets remained poorly studied in the context of MS immunopathology. We addressed these issues through the following specific aims: 
- Specific Aim 1. To assess CD8+ $\mathrm{T}_{\text {reg }}$ suppressive ability in healthy subjects and in relapsing-remitting MS patients during stable disease and during exacerbations through cross-sectional study.

- Specific Aim 2. To characterize the mechanisms of $\mathrm{CD} 8+\mathrm{T}_{\text {reg }}$ suppression.

Through these aims, we hoped to address the role of self-specific CD8+ Treg in pathology and during therapy as well as provide more fundamental insights into steady-state non-specific CD8+ Treg peripheral tolerance mechanisms. We hoped to pave the way for better therapeutic interventions for transplant, allergy and autoimmunity. 


\section{CHAPTER 2: METHODS AND MATERIALS}

\section{SUBJECT CHARACTERISTICS}

MS patients were recruited and gave written informed consent at the UT Southwestern Clinical Center for Multiple Sclerosis. Table 1 summarizes patient characteristics. 11 treatment-naïve adult clinically definite RRMS patients (McDonald criteria) with quiescent disease were recruited [302]. Exclusion criteria included pregnancy, HIV positivity, active cancer, other autoimmune, immunosuppressive, neurodegenerative conditions, clinical relapse or corticosteroid treatment within last 3 months, any history of disease-modifying immunomodulatory therapy. In addition, 9 treatment-naïve MS patients were recruited during an active acute clinical episode/relapse. 15 healthy subjects were recruited as controls (HC). All studies were approved by the UT Southwestern IRB according to Declaration of Helsinki principles. 
Table 1: Summary of Patient Characteristics

$\begin{array}{lllll} & \text { Healthy } & \text { RRMS: } & \text { MS: } & \text { MS: } \\ & \text { Controls } & \text { Quiescent } & \text { Acute } & \text { Exacerbation } \\ & (\mathrm{HC}) & (\mathrm{MS}) & \text { Exacerbation } & \text { Follow-up } \\ \text { Number of Subjects } & 15 & 11 & 9 & 4 \\ \text { Average age, y } & 44 & 40 & 45 & 44 \\ \text { (Range) } & (21-65) & (23-56) & (31-65) & (35-53) \\ \text { Sex (M/F) } & 5 / 10 & 2 / 9 & 3 / 6 & 2 / 2 \\ \text { Days from } & \text { N/A } & 599 & 8 & 81 \\ \text { Last Relapse } & & (90-2920) & (2-50) & (31-118) \\ \text { [Mean (Range)] } & & & & \end{array}$




\section{CELL PREPARATION AND BEAD SORTING}

PBMC were isolated from whole blood using Ficoll Hypaque (GE Healthcare Biosciences, Pittsburgh, PA) density gradient. Purified CD8+ T-cells were isolated using CD8+ Microbeads positive selection kit (Miltenyi Biotec, Auburn, CA) and AutoMacs separation, according to the manufacturer's instructions. $\mathrm{CD} 8+$ enriched populations were $>95 \% \mathrm{CD} 8+$ and $<0.1 \% \mathrm{CD} 4+$ by flow cytometric analysis. "Untouched" CD4+ T-cells were isolated using CD4 negative selection kits (Miltenyi Biotech). CD25+ T-cells were depleted from the purified CD4+ using CD25 Microbeads (Miltenyi Biotec). CD4+CD25- enriched populations were $>98 \% \mathrm{CD} 4+,<1 \% \mathrm{CD} 25+$, and $<0.1 \% \mathrm{CD} 8+$ by flow cytometric analysis. CD4+CD25+ enriched populations were $>98 \%$ CD4+ and $<0.1 \%$ CD8+. CD25 expression ranged from 40.5-73.8\%. The CD4+ and CD8+ T-cell-depleted PBMC population was irradiated with 3000 rads before being used as antigen-presenting cells (APC).

\section{CFSE STAINING}

To detect proliferative responses upon antigenic challenge, cells were stained with carboxyfluorescien diacetate succinimidyl ester (CFSE) (Invitrogen Molecular Probes, Eugene, OR), as described previously [245] [100]. Briefly, cells are suspended at $1 \times 10^{6}$ cells $/ \mathrm{mL}$ and incubated for 7 min at $37^{\circ} \mathrm{C}$ with $0.25 \mu \mathrm{M}$ CFSE (Invitrogen), then washed twice with media containing 5\% human serum.

\section{CMTPX STAINING}

Cell Tracker Red CMTPX (Invitrogen Molecular Probes) was used to stain putative regulatory cells. $\mathrm{CD} 8+$ and $\mathrm{CD} 4+\mathrm{CD} 25+$ suppressor cells, or 
CD4+CD25- negative control cells were marked with CMTPX, as described previously [106]. Briefly, cells were suspended at $1 \times 10^{6}$ cell/mL and incubated 15 min at $37^{\circ} \mathrm{C}$ with $700 \mathrm{nM} \mathrm{CMTPX}$, then washed twice with media containing 5\% human serum. The longer-wavelength CMTPX exhibits bright red fluorescence that is easily distinguished from that of green fluorescent probes, such as CFSE.

\section{FLOW CYTOMETRY-BASED SUPPRESSION ASSAY CULTURES}

Peripheral blood mononuclear cells (PBMC's) from healthy buffy coats and cord blood samples (where indicated) were separated using ficoll gradients. CD4+CD25- and CD4+CD25+ $\mathrm{T}$ cell populations were isolated using CD4 negative selection and CD25 positive selection magnetic microbead isolation kits from Miltenyi Biotec. Irradiated CD4 depleted populations were used as antigen presenting cells (APC) in all experiments. FOXP3 expression in CD4+CD25- T cells was induced by activation with soluble or plate bound anti-CD3/anti-CD28 or allostimulation by healthy APC's as indicated. FOXP3 expressing activated cells from varying time points were added back in a suppression assay to test their suppressive activity as described further. $1 \times 10^{6}$ CFSE-stained CD4+CD25- Tcells were used as responders in a $1 \mathrm{ml}$ culture. $1 \times 10^{6} \mathrm{CD} 4-$ and CD8- depleted PBMC were irradiated with 3000 rads and used as APC. In replicate cultures, varying ratios of CMTPX-stained suppressors $(\mathrm{CD} 8+$ or $\mathrm{CD} 4+$ cells where indicated) were added and cultured with various antigenic stimuli for 7 days in complete RPMI 1640 media containing 5\% human serum, 100 U/mL Penicillin, 
$100 \mu \mathrm{g} / \mathrm{mL}$ Streptomycin, and $0.92 \mathrm{mg} / \mathrm{mL}$ L-glutamine. Cells were washed and stained for flow cytometry, as described below.

\section{ANTIGENIC STIMULATION}

Pools of 15-mer peptides, overlapping by 10, spanning entire neuroantigenic proteins were used, as described previously [100]. These were used at $1 \mu \mathrm{g} / \mathrm{ml}$ final concentration for each peptide and covered myelin basic protein (MBP), proteolipid protein (PLP), myelin oligodendrocyte glycoprotein (MOG), myelin associated glycoprotein (MAG), oligodendrocyte myelin glycoprotein (OMGP) and $\alpha \beta$-crystallin (CRAB). In addition, whole bovine MBP (wbMBP) was also used at $20 \mu \mathrm{g} / \mathrm{ml}$. For control foreign antigens, we utilized pools of known CD4 and CD8 epitopes of CMV (5 and 14 peptides, respectively) as well as whole cytomegalovirus (CMV) (Microbix Biosystems, Ontario, Canada) and tetanus toxoid (TT) (Accurate Chemical \& Scientific Corp, Westbury, NY). $1 \mu \mathrm{g} / \mathrm{mL}$ anti-CD3 monoclonal antibody (OKT3) was used for mitogenic stimulation.

\section{OTHER REAGENTS}

Anti-CD3 (OKT3 Clone) was used at a concentration of $1 \mu \mathrm{g} / \mathrm{ml} / \mathrm{million}$ cells. Anti-CD28 (BD Biosciences) was used at $1 \mu \mathrm{g} / \mathrm{ml} / \mathrm{million}$ cells. Recombinant human IL-2 obtained from Invitrogen. Recombinant human TGF $\beta$, human IL-10, CTLA-4/Fc Chimera, anti-hIL2 (clone 5334), anti-hIL-2R $\alpha$ (clone 22722), antiTGF $\beta 123$ (clone 1D11) and anti-TGF $\beta$ were obtained from R\&D Biosytems Inc. CD45RO microbeads were used for CD45RO depletion (depletion greater than 95\%) to obtain a CD45RA enriched naïve and CD45RO enriched memory 
population. CD4 depleted enriched APC's were stained with the red fluorescent cell membrane dye PKH26 (Sigma) as per the manufacturers protocol. T CELL LINE GENERATION

We generated neuroantigen- and control antigen-specific CD8+ and CD4+ T-cell lines by bead-sorting CD8+ (or CD4+) T-cells after 1 week of in vitro PBMC stimulation, followed by repeated antigen-specific expansion with autologous APC (Figure 9). CD8+ T-cell lines were maintained with $25 \mathrm{IU} / \mathrm{mL}$ IL-2 (Peprotech, Rocky Hill, NJ), 10 ng/mL IL-7 (Peprotech), 1 ng/mL IL-12 (Peprotech), and $1 \mathrm{ng} / \mathrm{mL}$ IL-15 (Peprotech), as previously described [303, 304]. 
Figure 9: Growing antigen-specific $\mathbf{T}$ cell lines

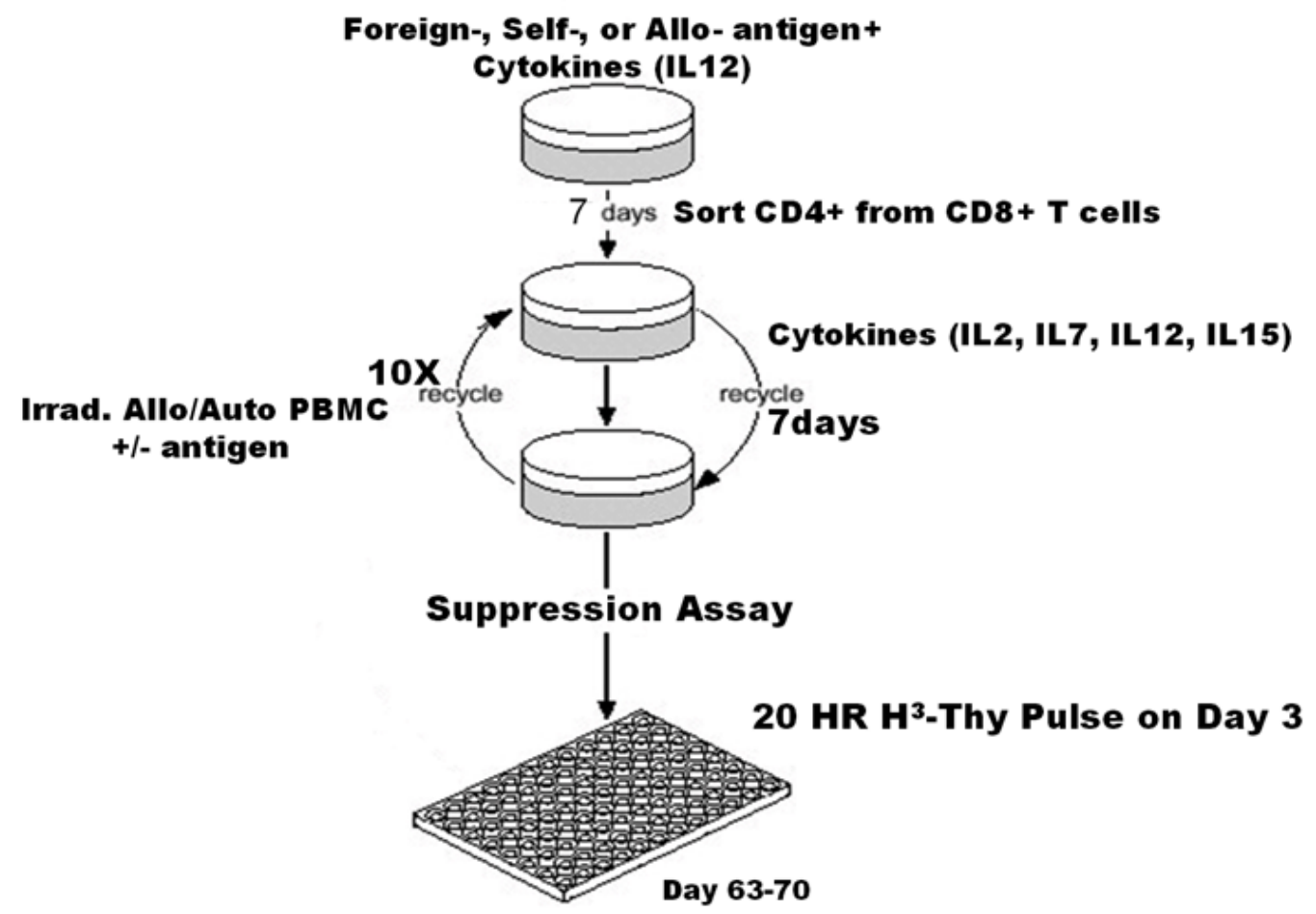




\section{FLOW CYTOMETRIC ANTIBODY STAINING}

On day 7 of in vitro stimulation, cells were washed with $0.1 \%$ (w/v) sodium azide/phosphate-buffered saline (Mediatech Cellgro). Cells were stained with anti-CD3-PE (BD Biosciences, San Jose, CA), anti-CD4-PECy5.5 (Invitrogen), anti-CD8-Pacific Blue (BD Biosciences), and anti-CD25-APC (BD Biosciences), then resuspended in 1\% paraformaldehyde (Electron Microscopy Sciences, Hatfiled, PA). Flow cytometric data were acquired on a 4-Laser, 17-color LSRII using FACSDiva software (Becton Dickinson). CFSE was detected in the FITC channel and CMTPX in the PE-Texas red channel on the LSR. Human and mouse FOXP3 staining kits from eBiosciences were used to stain for intracellular FOXP3. PCH101 and 236AE/7 anti-FOXP3-PE, AlexaFluor700 or Pacific Blue were used to stain for human FOXP3.

\section{DATA ANALYSIS}

Linear uncompensated data was transferred as FCS 3.0 files and analysed after compensation and transformation using FlowJo version 8.4.1 (TreeStar, Ashland, OR). Using Flowjo software (Treestar), putative Treg (CD4+CD25+, CD8+, and CD4+CD25- as a negative control) were CMTPX(high) and were gated out from flow cytometric analysis of CFSE-stained cells. Similar PKH-26 (Sigma-Aldrich, St. Louis, MO)-labeling techniques have been utilized for the purpose of excluding Treg from proliferative quantitation of $\mathrm{CD} 4+$ responder T-cells (Joosten, van Meijgaarden et al. 2007). T-cell activation and proliferation was quantified by the percentage of CD25(high) and CFSE(low) events among gated CD4+ (or CD8+) T-cells. Cut-offs for positive populations were determined by 
using either fluorescence minus one (FMO) staining for polychromatic flow cytometry, no stimulus background CFSE staining, or isotype control staining, as appropriate [305]. A "positive" T-cell response to antigen was defined as having (1) a response index (RI) greater than or equal to 1.5 and (2) a \%CD25+CFSElow response of the antigen-stimulated cells at least $1 \%$ greater than the $\%$ CD25+CFSElow response of the cells in the no antigen tube. Response index (RI) was the stimulated cells' \%CD25+CFSElow divided by 100 $\%$ CD25+CFSElow divided by the unstimulated cells' \%CD25+CFSElow divided by $100-\% C D 25+C F S E l o w$. If these criteria were unmet, absence of $\mathrm{T}$-cell response was indicated. For suppression assays, \% response was calculated by normalizing the 'responder only' proliferation to $100 \%$. \%Suppression was 100 minus \%response. CMTPX(high)CD8+ cells were analyzed for CD25+ expression. Stimulation index of the CD8 response was defined as the percentage of CD25+ cells with antigenic stimulus divided by percentage with no antigen.

\section{H THYMIDINE BASED ASSAYS}

Assays were performed in triplicate in 96-well plates using antigen-specific T-cell lines. 1x105 CD4+ line cells were cultured with 1x105 irradiated autologous PBMC in a total volume of $200 \mu \mathrm{l} /$ well, with or without indicated antigens. $1 \times 10^{5}$ CD8+ line cells were added to the cultures as suppressors. The cultures were pulsed with $3 \mathrm{H}$-thymidine on day 3 and harvested after 20 hours to measure proliferation in CPM, as previously described $[100,245]$. $\triangle$ CPM was calculated by subtracting background proliferation in the absence of antigen.

STATISTICAL ANALYSES 
Statistical tests were performed using Prism 5 (Graphpad Software, La Jolla, CA). Correlation regression and $\mathrm{t}$ tests were used to compute a two-tailed $\mathrm{P}$ value assuming a $95 \%$ confidence interval. $\mathrm{P}$ values $>0.05$ were not significant with "ns" notated where applied in figures. Likewise P values 0.01 to $0.05,0.001$ to 0.01 , and $<0.001$ were significant with “*”, “**”, and “***” notated respectively. $\mathrm{R}$ squared values were computed from non-transformed raw data with the use of non-linear regression, assuming a semi-log $\mathrm{X}$ line model (days since start of last relapse is plotted on a logarithmic $\mathrm{X}$ axis)

STANDARDIZATION OF MIXED LYMPHOCYTE REACTION (MLR)

Autologous reaction: $1 \times 10^{6} \mathrm{CD} 4+\mathrm{CD} 25(-)$ responder and $1 \times 10^{6} \mathrm{~T}$-cell depleted PBMC target cells from the same donor were co-cultured in $\mathrm{mL} \mathrm{H5}$. These acted as background control, and were also useful to determine the self-reactivity and pseudo-reactivity of responders in absence of true antigenic stimulus. Alloreaction: Target APC cells from another random donor (HLA-mismatched) were used to activate responders. Responder cell proliferation in MLRs was determined by CFSE. APC were irradiated (30Gy). Proliferation and activation in MLR were compared against autologous control on day 7 of culture

\section{MLR-BASED SUPPRESSION ASSAY}

Suppression of alloreactive CD4 stimulation was carried out in culture with varying CD4 to suppressor ratios including the following: 1:0.125, 1:0.25, 1:0.5, and 1:1. CD8 suppressors autologous to CD4 responders or the APC were used, as well as a third party donor. Donor origin is annotated in Figure 59 and Table 2. Similar to the previously described flow-based suppression assay, on day 7 , cells 
are stained with florescent antibodies and fixed with $1 \%$ paraformaldehyde. Suppression was determined by comparison to CD4 response in the absence of suppressors.

MICE

C57BL/6 (B6) female mice were purchased from Taconic (Hudson, NY) and the UT Southwestern Mouse Breeding Core Facility (Dallas, TX). SJL/J female mice were purchased from National Cancer Institute (Bethesda, MD). All mice were housed and bred in the UT Southwestern Medical Center Animal Resource Center and used according to approved IACUC protocols.

\section{MOUSE IMMUNIZATION}

Six to 8 week-old C57BL/6 mice were immunized subcutaneously at two injection sites with $200 \mu \mathrm{g}$ MOG35-55 (MEVGWYRSPFSRVVHLYRNGK, UT Southwestern Protein Chemistry Technology Center) emulsified in CFA supplemented with $4 \mathrm{mg} / \mathrm{ml}$ Mycobacterium tuberculosis (MTB, H37Ra, Difco). Ovalbumin peptide 323-339 (OVA323-339, ISQAVHAAHAEINEAGR) was used as a peptide control. On days 0 and 2 post-immunization, $250 \mathrm{ng}$ of pertussis toxin (PTX, List Biological Laboratories) was administered intraperitoneally in $100 \mu 1$ of phosphate buffered saline (PBS).

\section{MURINE FLOW-BASED SUPPRESSION ASSAY}

Splenocytes from immunized mice were harvested. CD8+ cells were magnetically separated using a negative selection protocol (Miltenyi Biotech, Germany) to recover "untouched" CD8+ cells (>90\%). Irradiated splenocytes from naïve or immunized mice were used as APC at a ratio of 1:1 (CD4+ T cells:APC). Cells 
were harvested on glass fibers mats and counted using a Betaplate counter (Wallac, Gaithersburg, MD). Proliferation assays were performed using a carboxyfluorescein succinimidyl ester (CFSE)-dilution assay as described previously. Splenocytes were harvested and used either in bulk proliferation assays or as a source for CD8+ cells, which were isolated using a negative selection magnetic bead protocol. Bulk splenocytes or "untouched" CD8+ cells were suspended at $1 \times 10^{6}$ cells $/ \mathrm{ml}$ in PBS and incubated at $37{ }^{\circ} \mathrm{C}$ for 7 min with 0.25 $\mu \mathrm{M}$ CFSE, followed by addition of serum and two PBS washes. Subsequently, CD8+ cells were suspended at $0.5-1 \times 106 / \mathrm{ml}$ of media. On day 5, cells were washed with staining buffer and labeled with phycoerythrin (PE)conjugated-anti-CD8 and allophycocyanin (APC)-conjugated-anti-CD4 antibodies (Caltag/Invitrogen, Carlsbad, CA). After incubation for 30 minutes at $4{ }^{\circ} \mathrm{C}$, cells were washed and fixed in $1 \%$ paraformaldehyde (PFA, Electron Microscopy Sciences, Hattfield, PA). Flow cytometric data were acquired on BD LSR II flow cytometer using FACSDiva software. For analysis, FlowJo (Treestar, Ashland, OR) software was used to gate on lymphocytes and further on the CD4+ CD8- or CD8+ CD4- T cell subsets.

\section{SHRNA KNOCKDOWN OF ACTIVATED AND NATURAL FOXP3}

Human FOXP3 specific lentiviral particles and ShRNAs were obtained from Santa Cruz biotechnology (CA, USA). CD4+CD25- (Treg depleted) cells were thawed and infected with FOXP3 shRNA lentivirus or control (scrambled) shRNA with polybrene (Sigma-Aldrich Life Sciences, St. Louis, MO) for 24 hours, then washed and resuspended with fresh autologous APC. After an 
additional 24 hours, CD4+ cells were stimulated with anti-CD3 plus puromycin selective antibiotic, concentration determined per standard curve killing assay. After 48 hours, CD4+ cells were washed and resuspended with more APC, antiCD3, and puromycin. After 48 hours, CD4+ cells were intra-cellularly stained with FOXP3 or set up in 7 day flow-based suppression assay with autologous responders as described earlier. 


\section{CHAPTER 3: RESULTS}

CD8+ T CELLS IN MULTIPLE SCLEROSIS

MULTIPLE SCLEROSIS PATIENTS AND HEALTHY CONTROL SUBJECTS

\section{SHARE SIMILAR T CELL RESPONSES}

Most prior studies comparing CNS-specific T-cell responses between MS patients and healthy subjects have employed proliferation assays using bulk PBMC. Using CFSE-based flow cytometric proliferation assays, my lab has shown a high prevalence of $\mathrm{CD} 4+$ and $\mathrm{CD} 8+\mathrm{T}$-cell responses to neuroantigens in both healthy subjects and MS patients, with some functional differences [202]. In the current study, I performed CFSE assays using magnetically purified CD4+CD25- and CD8+ cells. I observed that, similar to bulk PBMC, purified populations of CD4+CD25- and CD8+ T-cells from treatment-naïve MS patients (MS) and healthy control subjects (HC) showed similar responses to neuroantigens, foreign antigens and mitogenic (anti-CD3) stimulation ( 
Figure 10-Figure 11). Figure 10 shows examples of CD4+ T cell proliferation from representative $\mathrm{HC}$ and MS subjects, whereas Figure 11 shows cumulative data from $15 \mathrm{HC}$ and $11 \mathrm{MS}$, representing 50 and 37 detectable CNSspecific CD4 responses and 25 and 13 CD8 responses, respectively. 
Figure 10: Multiple sclerosis patients and healthy control subjects share similar T-cell responses
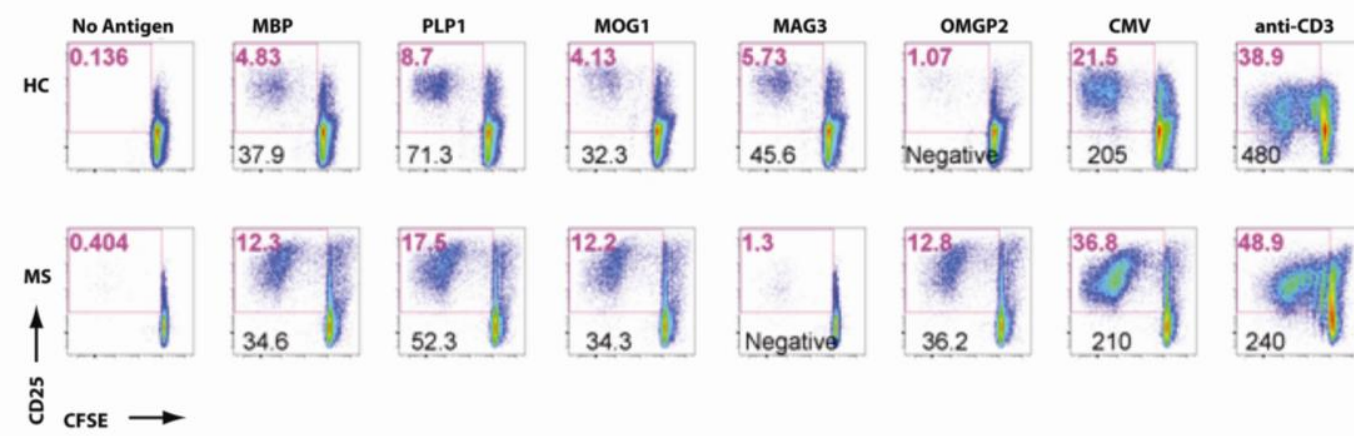

CFSE-based proliferation assays were performed on purified CD4+CD25- or CD8+ T-cells from $15 \mathrm{HC}$ and $11 \mathrm{MS}$ patients. Representative responses from CD4+ T-cells from a single HC (top row) and single MS patient (bottom row) are shown, with CFSE on $\mathrm{X}$-axis and CD25 on the $\mathrm{Y}$-axis. Various stimuli are indicated above each column. The numbers in red toward the top of each dotplot indicate the \%CD25+/CFSE(low) (activated/proliferating) cells, representing the response. Numbers in black toward the bottom represent the response index (RI), calculated based on background proliferation in the absence of any stimulus. "Negative" represents lack of a response, based on criteria described in the methods. 
Figure 11: Multiple sclerosis patients and healthy control subjects share similar T-cell responses

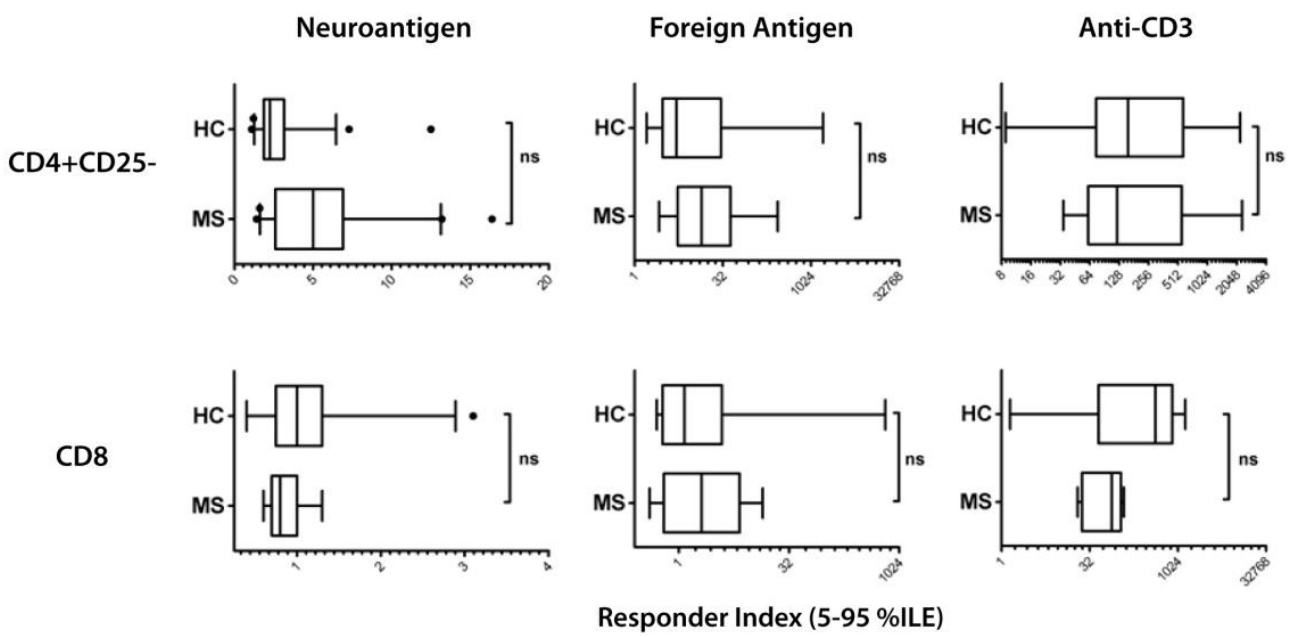

Cumulative results from $15 \mathrm{HC}$ and $11 \mathrm{MS}$ patients (9 neuroantigenic responders) are shown as RI for both CD4 responses (top row) and CD8 responses (bottom row), stimulated with neuroantigens, foreign antigens or anti-CD3 (as indicated). These results represent 85 and 60 positive assays with neuroantigens performed on $\mathrm{HC}$ and MS, respectively. P values $>0.05$ were not significant with "ns" notated where applied in figures. 
I also performed parallel assays using bulk PBMC versus purified CD4+CD25- T-cells [i.e., in the absence of CD8+ T-cells and CD4+CD25+ regulatory T-cells], predominantly using HC PBMC. I observed that depletion of CD8+ T-cells and CD25+ cells, resulted in a significant increase in CD4+ T-cell responses to neuroantigens, but not to control foreign antigens like CMV or TT ( 
Figure 12). This suggested that CNS-specific CD8+ T-cells may possess immune suppressive ability. We therefore set about designing a novel suppression assay. 
Figure 12: Depletion of CD8+ $T$ cells and CD25+ cells increased CD4+ T-cell responses to neuroantigens, but not to control foreign antigens

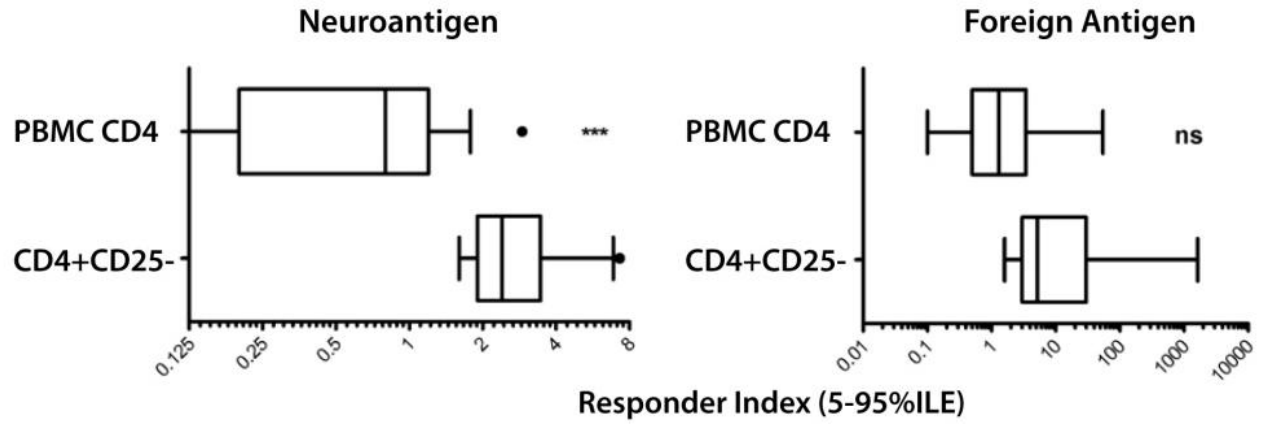

From 9 HC, CFSE-based proliferation assays were performed on both bulk PBMC as well as sorted CD4+CD25- T-cells. Cumulative results from gated CD4 responses from each condition are shown as RI. *** indicates significant elevation of neuroantigen-specific responses $(\mathrm{p}<0.001)$, whereas foreign antigenspecific responses were not significantly different (ns). 


\section{NOVEL FLOW-CYTOMETRY-BASED SUPPRESSION ASSAY}

The assay system utilized in my studies took advantage a proliferation dye (CFSE), a cellular tracking dye (CMTPX) and overlapping antigenic peptide pools to monitor neuroantigen-specific CD8+ T-cell suppressive ability (Figure 13, Figure 14, Figure 15). This assay has excellent sensitivity and specificity for detecting functional antigen-specific suppressive ability, by allowing the exclusion of suppressor populations from the analysis. Moreover, it allows an unbiased characterization of T-cell suppressive ability without limited range of HLA haplotype or epitopes. Finally, the assay also enables separate concurrent characterization of $\mathrm{CD} 4+$ and $\mathrm{CD} 8+\mathrm{T}$ cell responses within the same culture. Thus, this unique approach allowed me to discover and quantify this novel autoregulatory function of CNS-specific CD8+ T-cells and activated regulatory $\mathrm{T}$ cell populations described below.

I hypothesized that CNS-specific CD8+ T cells may possess regulatory function. To test this hypothesis, I first took advantage of a sensitive flow cytometry-based suppression assay [90] to measure suppressive ability of bulk autologous CD8+ T-cells (Figure 14A). This assay measured the proliferation and activation of CFSE-stained CD4+CD25- responder T-cells. Putative suppressor cells were stained with a tracker dye, CMTPX [306], allowing their exclusion from the analysis. CMTPX-stained CD4+CD25+ (positive control), CD8+ or CD4+CD25- (negative control) T-cells were added in increasing numbers and their effect on responder proliferation was quantified, by normalizing to the RI of CD4+CD25- T-cells (treated as $100 \%$ proliferation or $0 \%$ suppression). 
Representative dotplots of anti-CD3-stimulated assays are shown in Figure 14, with cumulative $\%$ proliferation shown in Figure 15 and \% suppression from a single responder to suppressor ratio in Figure 16. Using anti-CD3 stimulation, we observed consistent suppressive activity in the CD4+CD25+ and CD8+ populations, while CMTPX-stained CD4+CD25- T-cells did not significantly dampen pan-stimulated CD4+CD25- T-cells (negative control). Interestingly, non-fractionated CD8+ T-cells showed greater suppressive capacity than CD4+CD25+ T-cells, a fraction known to contain regulatory T-cells. 
Figure 13: Basic design and gating strategy of flow-based suppression assay

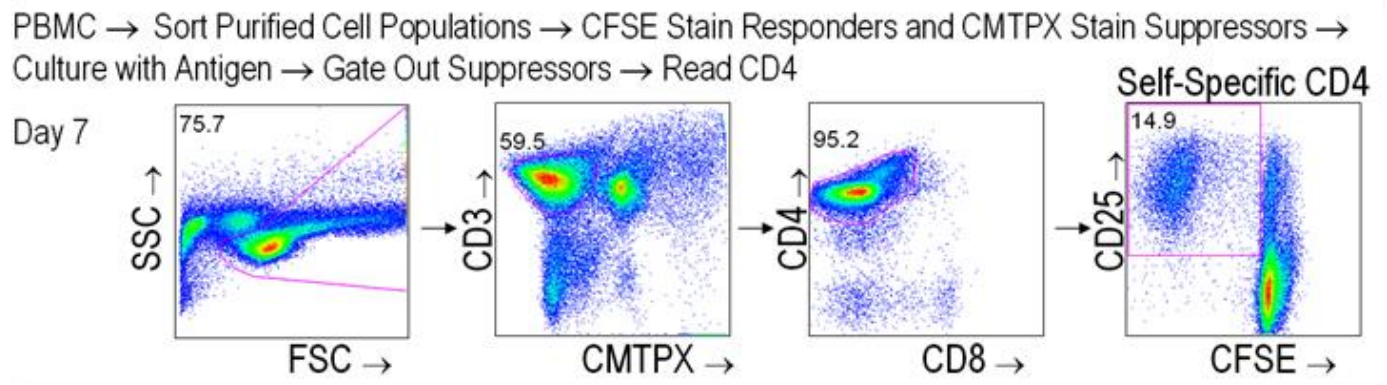

PBMC were magnetically-sorted into CD4+CD25- and CD8+ cells, and then stained with CFSE and CMTPX respectively. Co-cultured with CD8+ suppressors, CD4+ responders were cultured in RPMI containing 10\% human serum for seven days with PLP antigen and autologous APC. All cells were stained for FACS analysis, and representative pseudo-color plots were gated on CD4+ T cells. 
Figure 14: Flow-based suppression Assay is validated: CD4+CD25+ Tregs and CD8+ T-cells suppress anti-CD3-stimulated CD4+ T-cells, but not CD4+CD25- cells

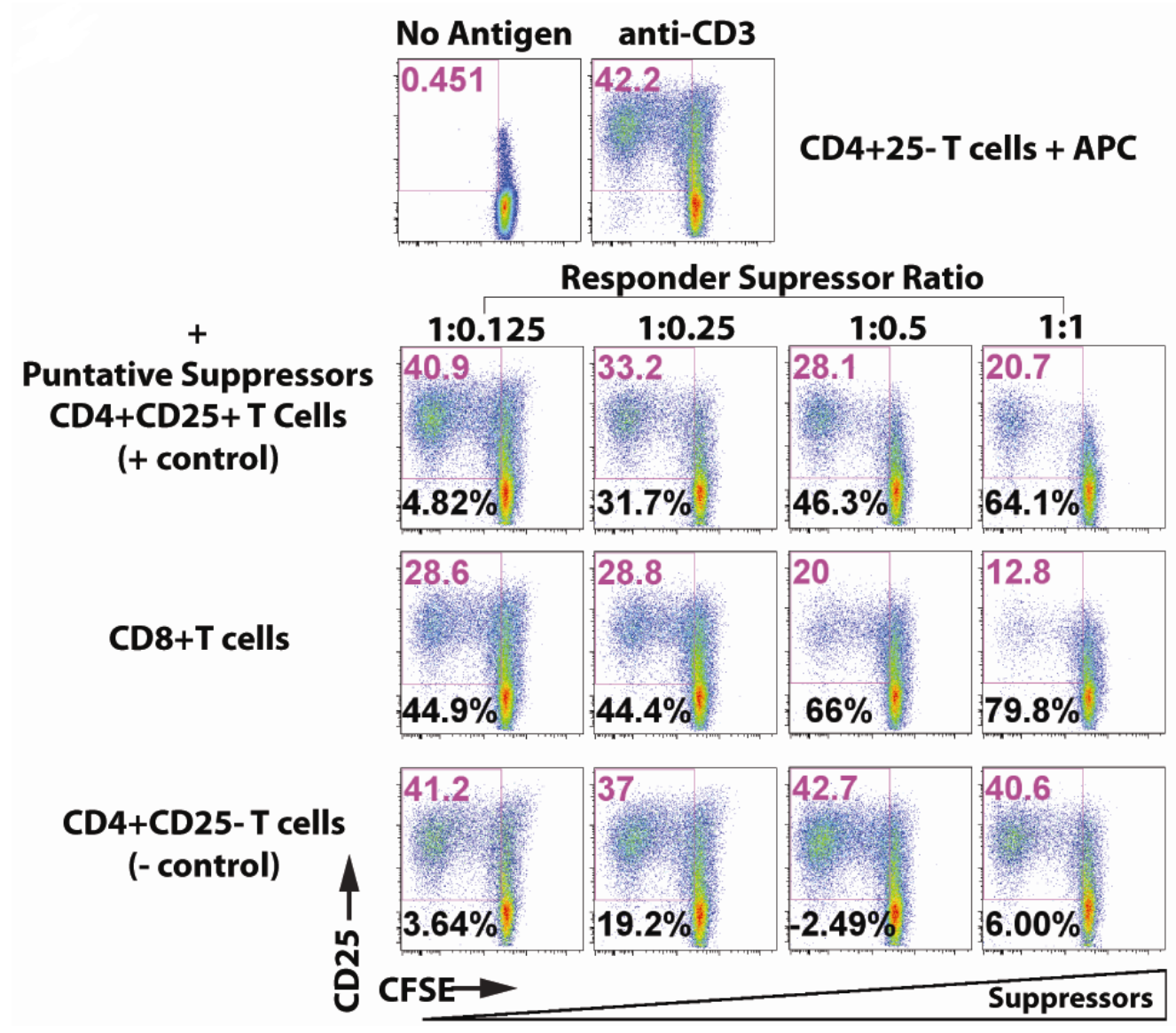

CFSE-stained healthy ex vivo purified CD4+CD25- T-cells were used as responders in anti-CD3-stimulated suppression assays. Dotplots from a single representative experiment demonstrate CFSE on the X-axis and CD25 expression on Y-axis. Indicated in red at the top of each dot plot is the gated percentage of CD25+/CFSE-low cells (activated and proliferating), representing the "response". Indicated in black in the lower left is the calculated \%suppression, based on normalizing to the anti-CD3-mediated response in the absence of suppressors (top row). Indicated to the left of the bottom three rows are the CMTPX-stained cell populations used as suppressors at the indicated ratios over each column. The results are representative of 15 flow-based suppression assays from 15 healthy controls. 
Figure 15: Flow-based suppression Assay is validated: CD4+CD25+ Tregs and CD8+ T-cells suppress anti-CD3-stimulated CD4+ T-cells, but not CD4+CD25- cells

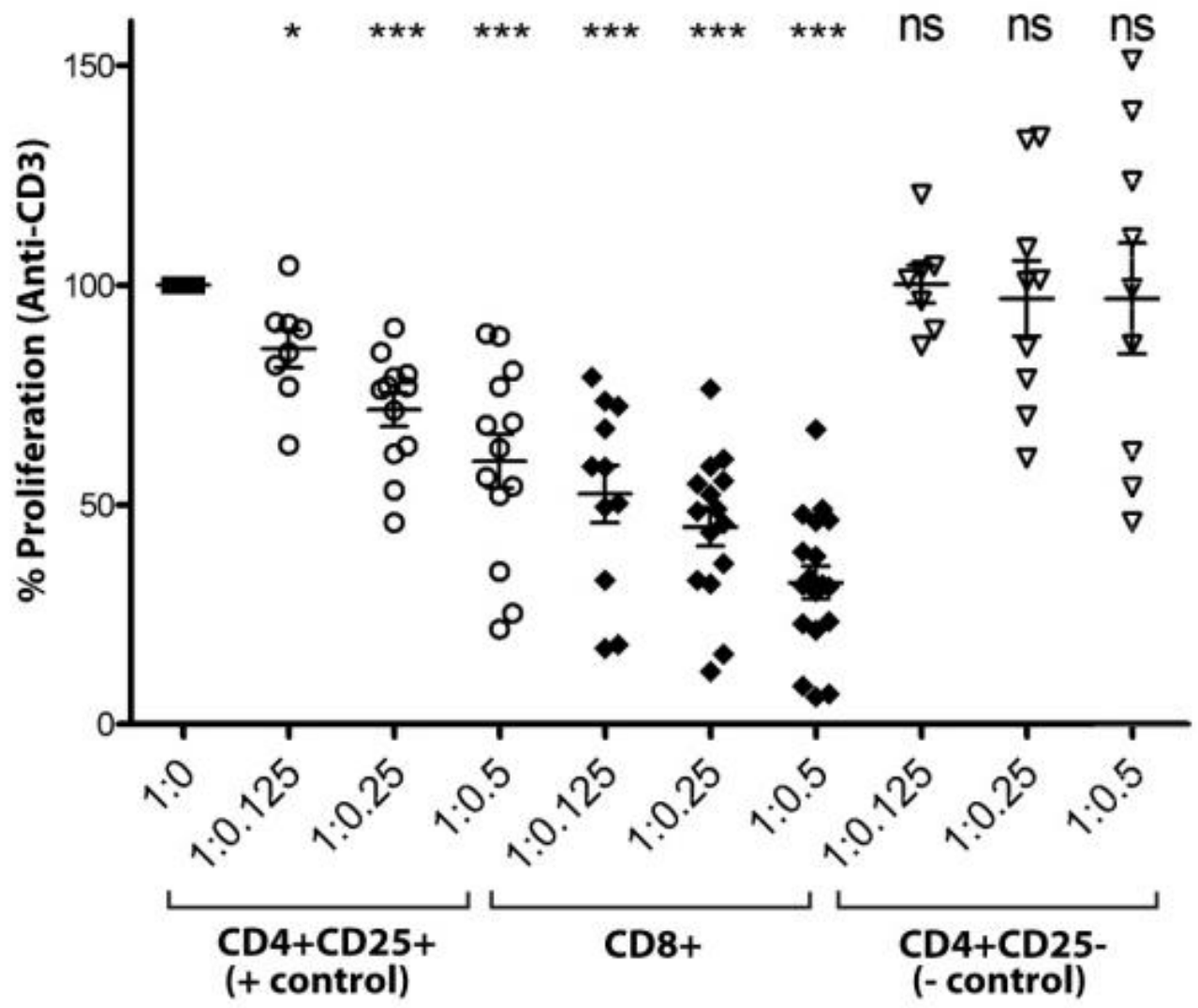

Responder:Suppressor Ratio

Cumulative results from suppression assays from 15 healthy controls are displayed as percent proliferative response normalized to the response without suppressors (defined as 100\%), indicated as 1:0. Open circles represent the response in the presence of increasing numbers of CD4+CD25+ T-cells (positive controls), closed diamonds for bulk CD8+ T-cells and open triangles for CD4+CD25- T-cells (negative controls). $\mathrm{P}$ values $>0.05$ were not significant with "ns" notated where applied in figures. Likewise P values 0.01 to 0.05 , and $<0.001$ were significant with “*” and "**** notated respectively. 
Figure 16: Anti-CD3-stimulated and neuroantigen-specific CD8+ T-cells suppress CD4+ T-cells

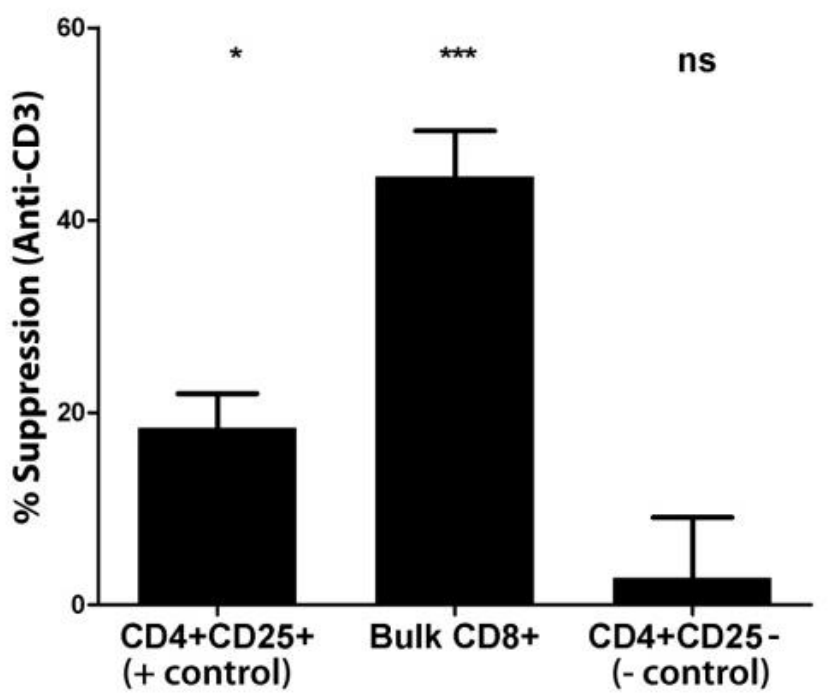

Results from Figure 15 are represented as \% suppression at a single responder: suppressor ratio (1:0.25). P values $>0.05$ were not significant with "ns" notated where applied in figures. Likewise $\mathrm{P}$ values 0.01 to 0.05 , and $<0.001$ were significant with “*”, and “***” notated respectively. 
CD8+ T-CELLS SPECIFIC FOR CNS AUTOANTIGENS, BUT NOT THOSE SPECIFIC FOR CONTROL FOREIGN ANTIGENS, SUPPRESS CD4+ T-CELL PROLIFERATION

I then sought to evaluate the suppressive ability of CD8+ T-cells in cultures stimulated with specific antigens by conducting suppression assays using a panel of CNS and control antigens. Positive CD4 T-cell responses to specific antigens were screened with MBP, MOG, PLP, MAG, OMGP, CMV, and TT and suppression was quantified. Figure 17 shows representative responses from one $\mathrm{HC}$, where the addition of increasing numbers of CD8+ T-cells suppressed the proliferation and activation of neuroantigen-stimulated responses, in contrast to foreign-antigen-stimulated responses. Figure 18 shows cumulative data from 15 HC, demonstrating consistent dose-dependent suppression in neuroantigenstimulated cultures (left), contrasting with lack of consistent suppression in response to foreign antigens (right), which in many cases led to enhanced proliferation of the responders [denoted as "negative suppression"]. This suggested that neuroantigen-specific CD8+ T-cells obtained ex vivo possessed immune suppressive ability, whereas foreign antigen-specific ones did not show consistent suppression. 
Figure 17: Neuroantigen-specific CD8+ T-cells suppress CD4+ T-cells
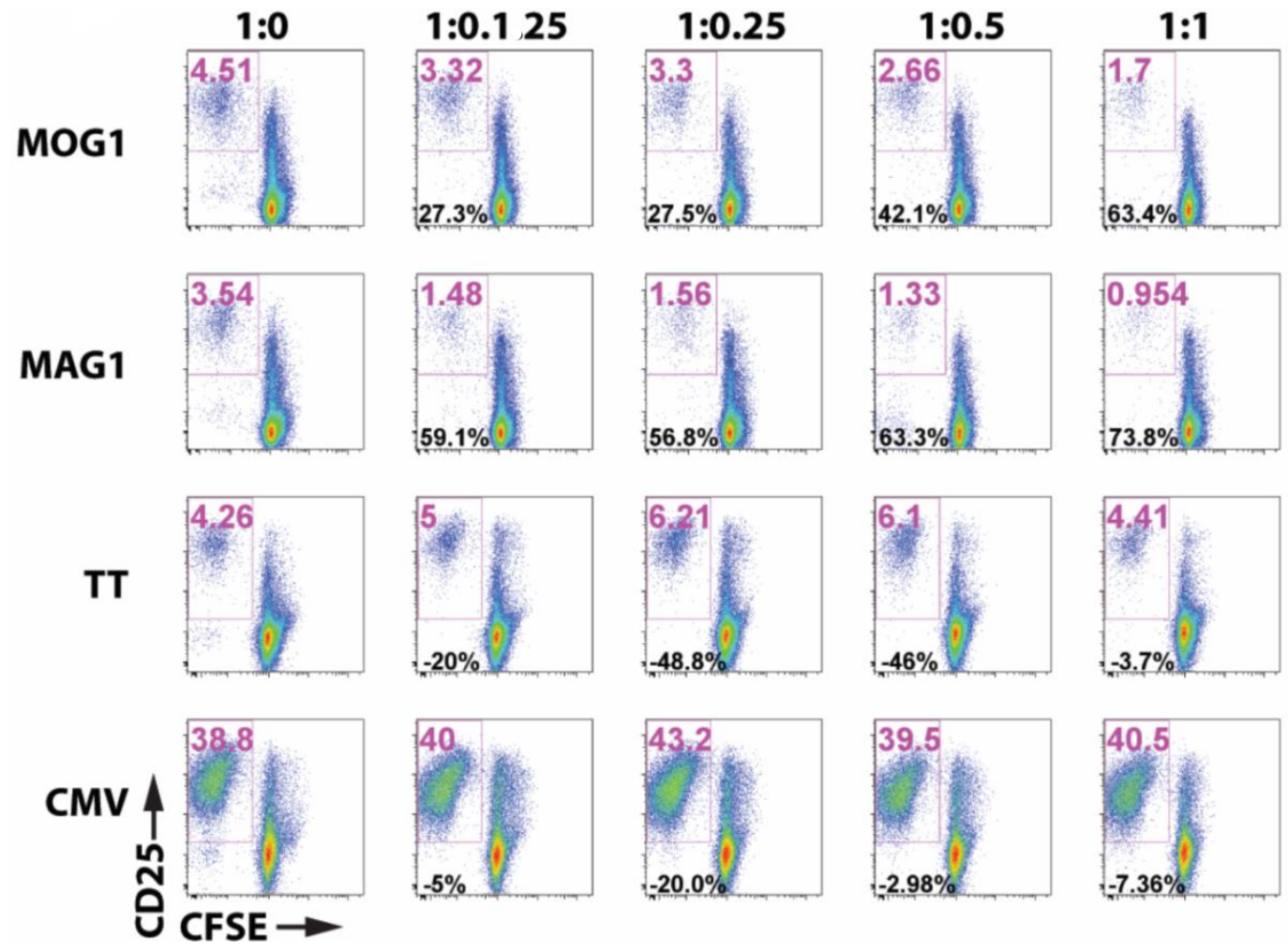

Representative dotplots from a single subject demonstrate CD8-mediated suppression assays in the presence of neuroantigens (MOG1, MAG1) and foreign antigen (TT, CMV). The left column represents CD4+CD25- responders only, where positive responses were selected to evaluate suppression. The right three columns contain increasing numbers of CMTPX-stained bulk CD8+ T-cells, with $\%$ proliferation and $\%$ suppression indicated. 
Figure 18: Neuroantigen-specific CD8+ T-cells suppress CD4+ T-cells
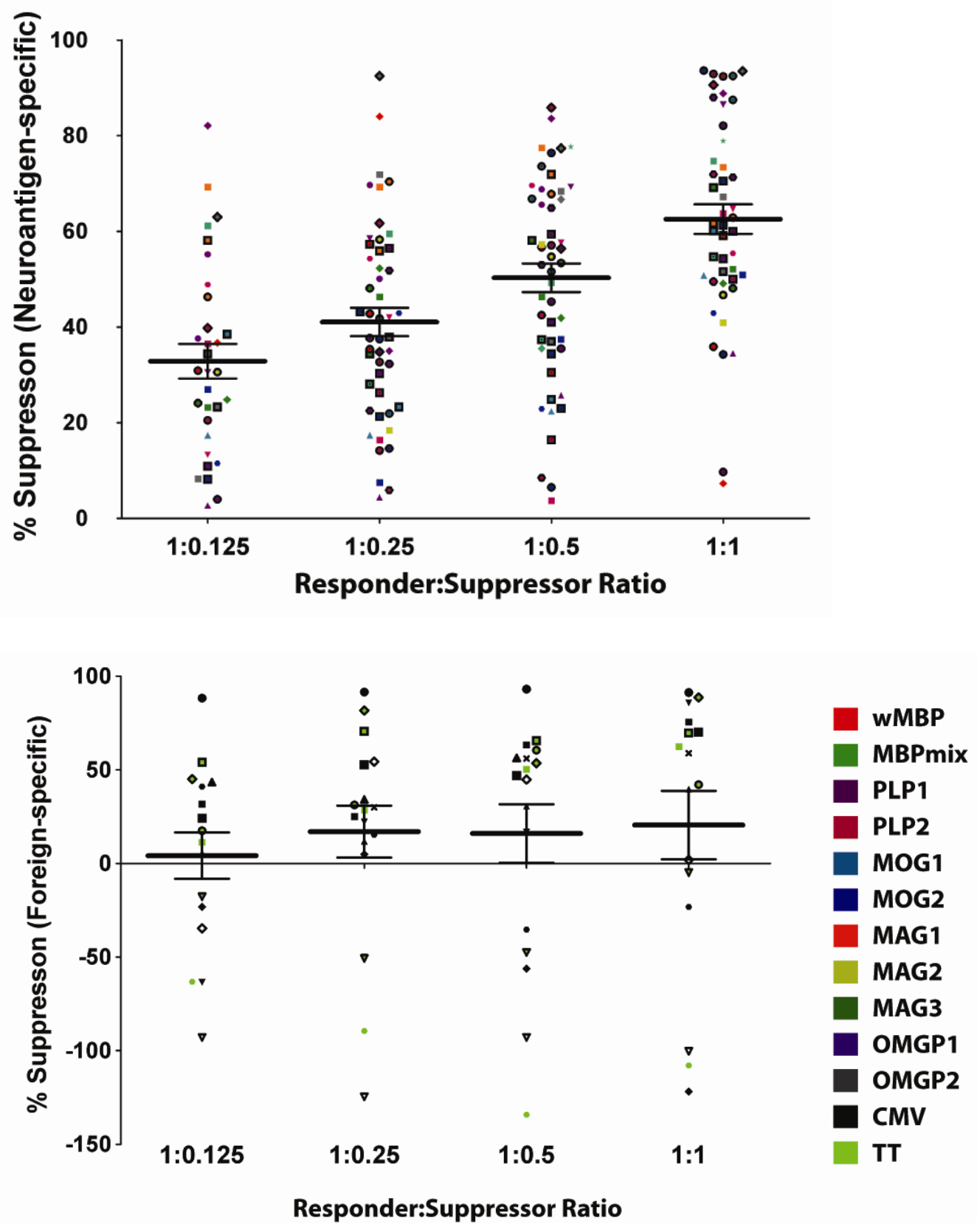

Cumulative results are shown from 67 suppression assays. Data points represent neuroantigen- (top) and foreign antigen-(bottom) specific \% suppression. Each of 15 subjects is indicated by a different shape. Neuroantigen or foreign antigen used in the suppression assay is indicated by the color legend at right (for some proteins, multiple pools were made to limit the number of peptides in each pool, as described previously [202]. 
CNS-SPECIFIC CD8+ T-CELLS REQUIRE STIMULATION WITH COGNATE ANTIGEN FOR SUPPRESSIVE ACTIVITY

In the experiments above, the antigens added to the bulk culture presumably stimulated both the CD4+ T-cells and CD8+ T-cells. Thus, it was possible only to test the effect of neuroantigen-stimulated CD8+ T-cells on CD4+ T-cells stimulated by the same antigenic peptides. To ascertain that these results were based on cognate antigen-specific recognition, I generated over thirty-four CD4+ and CD8+ T-cell lines, using PBMC from 8 HC. Specificity was confirmed by ${ }^{3} \mathrm{H}$-thymidine uptake, showing reactivity to the intended antigen but not to other CNS or foreign antigens (Figure 19). 
Figure 19: Antigenic specificity of $T$ cell lines

CMV CD8

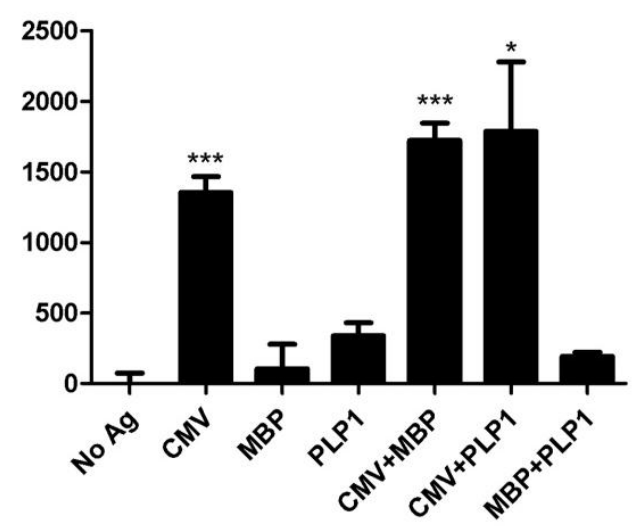

MBP CD8

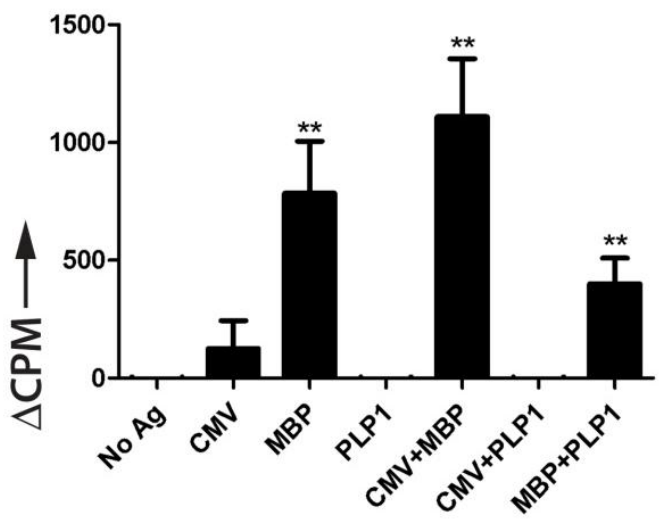

MBP CD4

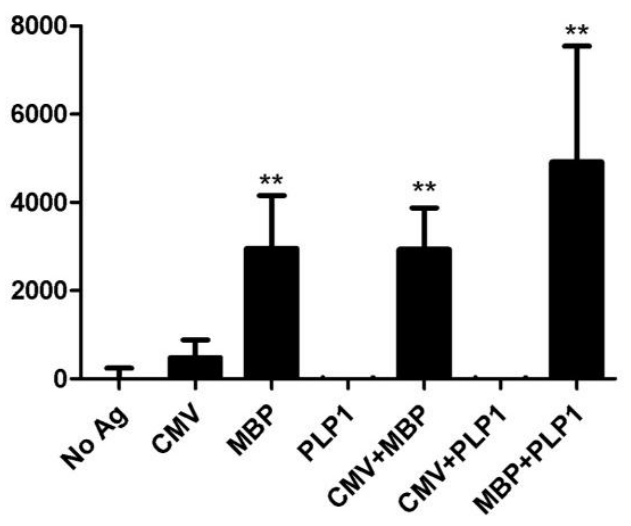

PLP CD8

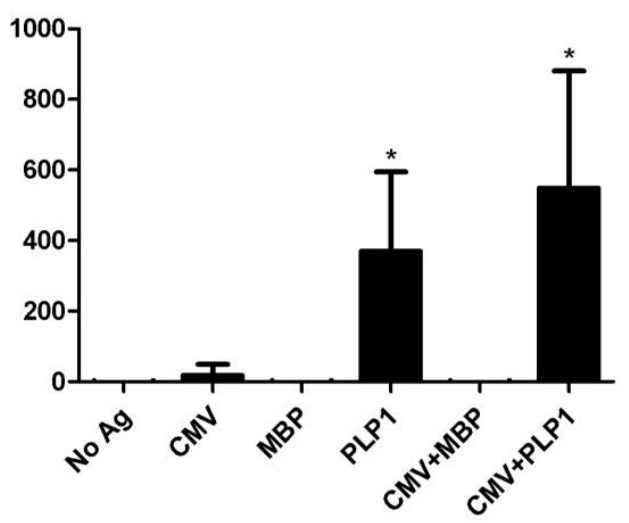

\section{Antigen}

CD4+ and CD8+ T cell lines of multiple specificities were grown from PBMC, as described in methods. Antigenic specificity was confirmed using 3H-Thymidine assays, before using these lines in suppression assays (Figure 14). These bar graphs show $\triangle \mathrm{CPM}$ (counts per minute, background subtracted) of representative lines, confirming response to the desired antigen $(*)$ but not other antigens. These results are representative of $32 \mathrm{~T}$ cell lines derived from $8 \mathrm{HC}$. $\mathrm{P}$ values $>0.05$ were not significant with "ns" notated where applied in figures. Likewise P values 0.01 to $0.05,0.001$ to 0.01 , and $<0.001$ were significant with “*”, “**”, and "***" notated respectively. 
Using these lines, I performed autologous ${ }^{3} \mathrm{H}$-thymidine-based suppression assays, culturing CNS- or foreign antigen-specific CD4+ T-cells alone or in the presence of CNS- or foreign antigen-specific CD8+ T-cells in various combinations. These cultures contained APC with antigens that would stimulate just the CD4+ T-cells or both CD4+ and CD8+ T-cells (Figure 20). Figure 20A shows a single MBP-specific CD4+ T-cell line, cultured with autologous MBP-, PLP- or CMV-specific CD8+ T-cells. When cultured in the absence of any CD8+ T-cells, the MBP-specific CD4+ T-cells showed a similar proliferative response to stimulation by $\mathrm{MBP}, \mathrm{MBP}+\mathrm{CMV}$ or $\mathrm{MBP}+\mathrm{PLP}$. The addition of MBPspecific CD8+ T-cells to the MBP-stimulated cultures resulted in robust suppression of the response. Importantly, the addition of PLP- or CMV-specific CD8+ T-cells did not affect cultures stimulated only by MBP. However, when PLP antigen was added, the PLP-specific CD8+ T-cells suppressed MBP-specific CD4 proliferation. Most interestingly, this was not true of CMV-specific CD8+ T-cells. In cultures containing CMV peptides and CMV-specific CD8+ T-cells, no significant suppressive effect was exerted. Figure 20B demonstrates an example of a CMV-specific CD4+ T-cell line, in combination with autologous CMV-specific or MBP-specific CD8+ T-cells. Again, in contrast to CMVspecific CD8+ T-cells, MBP-specific CD8+ T-cells had a suppressive effect in the presence of their cognate antigen. Thus, similar to bulk cultures (Figure 17, Figure 18), antigen-specific lines confirmed that neuroantigen-specific CD8+ Tcells had robust immune suppressive properties compared to foreign antigenspecific CD8+ T-cells and required the presence of cognate antigen. 
Figure 20: Activated neuroantigen-specific CD8+ T-cells suppress CD4+ Tcells

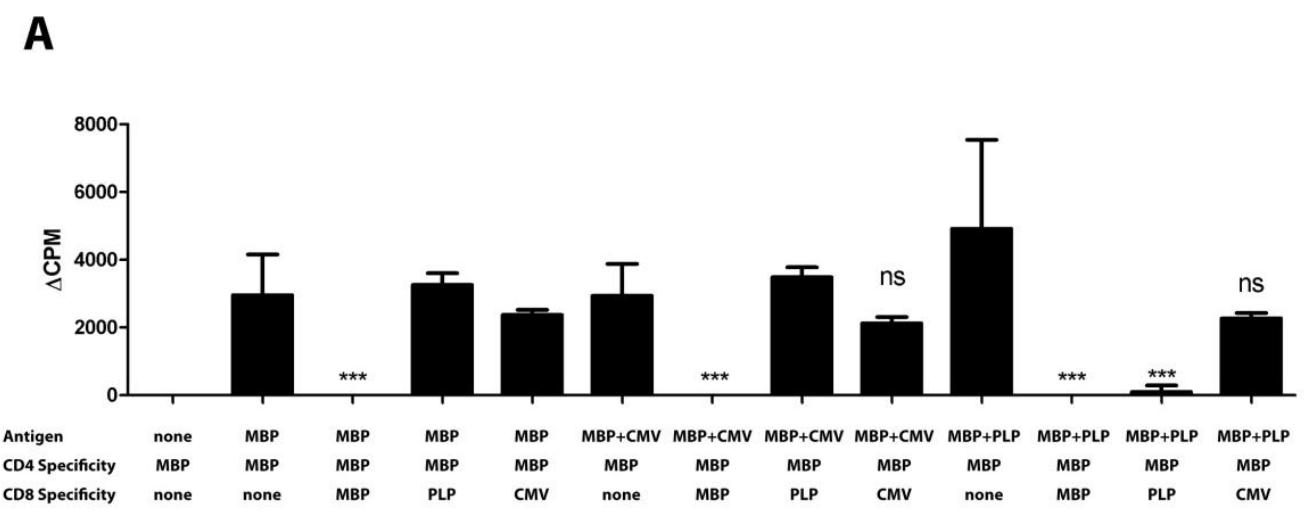

\section{B}

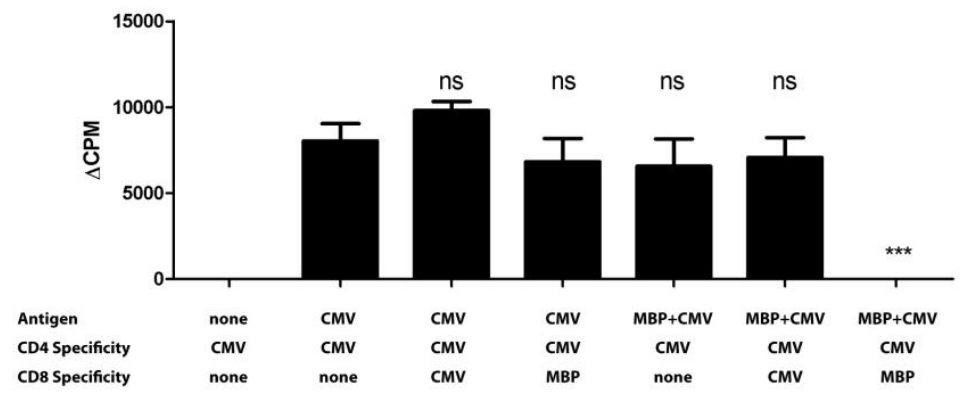

Responder CD4+ T-cell lines were cultured with APC and indicated antigens in the presence or absence of the indicated CD8+ T-cell lines. ${ }^{3} \mathrm{H}$-Thymidine-based proliferation assays were performed. Panel A shows $\triangle \mathrm{CPM}$ (background subtracted) from a single MBP-specific CD4+ line and Panel B shows a CMVspecific CD4+ line. The results are representative of 8 independent assays, each repeated twice, with lines obtained from 8 different HC. Further illustration provided in Figure 42. P values $>0.05$ were not significant with "ns" notated where applied in figures. Likewise $\mathrm{P}$ values $<0.001$ were significant with “**** notated respectively. 
CNS-SPECIFIC SUPPRESSIVE ABILITY IS SIGNIFICANTLY DIMINISHED DURING ACUTE EXACERBATION OF MS AND RECOVERS DURING REMISSION

I then postulated that neuroantigen-specific CD8+ T-cell suppressive ability may be relevant in MS and may influence the clinical disease course. Several studies by others and my lab have demonstrated a global deficit in regulatory CD4+CD25+ [243, 244, 307] or CD8+ T-cell function in MS [104, 126, 308]. To test the possibility that CNS-specific suppressive ability has a bearing on MS clinical presentation, I compared flow-based suppression assays on PBMC from $15 \mathrm{HC}, 11$ treatment-naive RRMS patients (quiescent MS) and 9 treatment-naïve MS patients during an acute exacerbation (Figure 21, Figure 22, Figure 23). CD8+ T-cells from HC and quiescent MS patients showed similar neuroantigenspecific suppressive ability (Figure 21, Figure 22). Interestingly, CD8+ T-cells obtained during an acute clinical episode showed significantly lower neuroantigen-specific suppressor ability, whether viewed as suppression stimulated by independent multiple antigens (Figure 22) or as a mean neuroantigen-specific suppression per subject (Figure 23). This corroborated with a global CD8 suppressor deficit, demonstrated in anti-CD3-stimulated suppressor assays (Figure 24), whereas none of the patients showed significant foreign antigen-specific CD8+ suppressor ability (Figure 25). 
Figure 21: Neuroantigen-specific suppressive ability is deficient during acute MS exacerbation
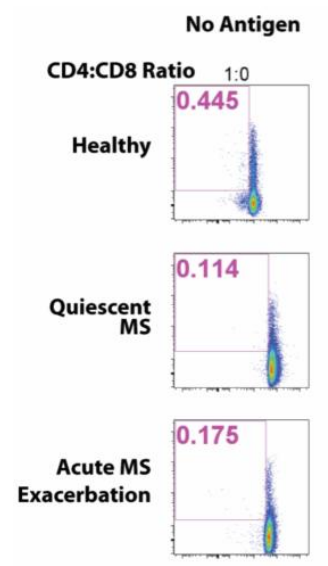
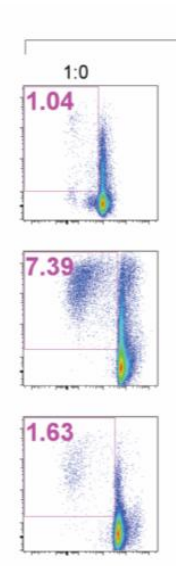

MOG1
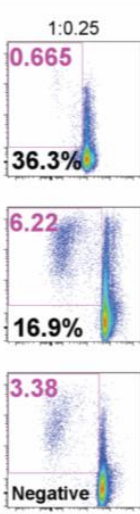
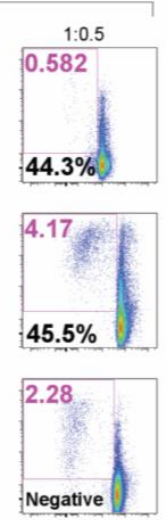
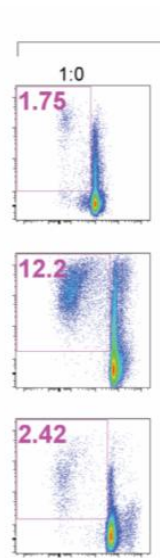
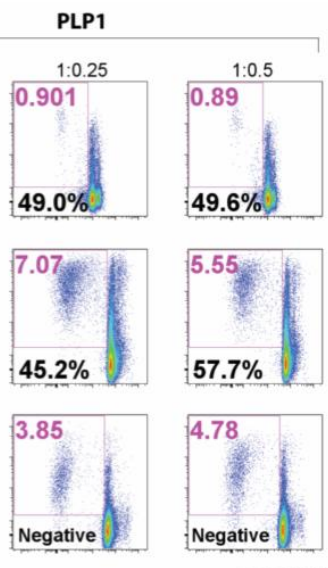

Ex vivo-purified, CFSE-stained CD4+CD25- T-cells from $\mathrm{HC}$, quiescent $\mathrm{MS}$ patients or MS patients suffering from an acute exacerbation were used as responders in autologous suppression assays. Dotplots display CFSE vs. CD25 from representative subjects responding to two neuroantigens (MOG-pool 1 and PLP-pool 1) in the absence of suppressor cells (1:0) or with CD8+ T-cells added at indicated ratios. Red numbers at the top of each dotplot represent proliferative response, whereas the black numbers represent the calculated \%suppression. This is representative $15 \mathrm{HC}, 11$ quiescent MS patients (9 responders) and 9 acute MS exacerbation patients (6 responders), equivalent to 50, 47, and 37 flow-based suppression assays, respectively. 
Figure 22: Neuroantigen-specific suppressive ability is deficient during acute MS exacerbation

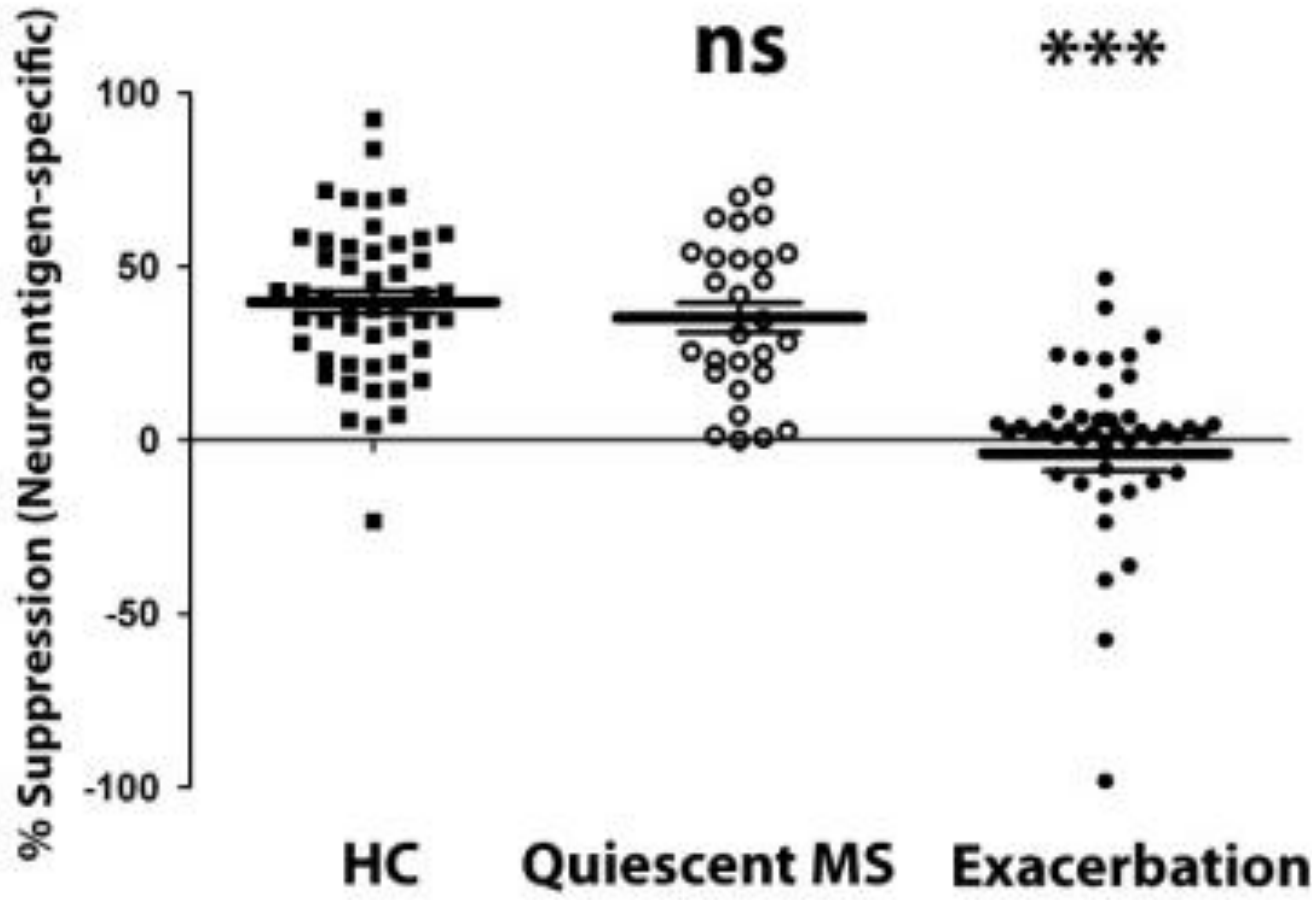

Panels show cumulative \% suppression data at the 1:0.25 responder:suppressor ratio from assays containing neuroantigens, foreign antigens or anti-CD3, as indicated. $\mathrm{P}$ values $>0.05$ were not significant with " $\mathrm{ns}$ " notated where applied in figures. Likewise $\mathrm{P}$ values $<0.001$ were significant with “***" notated respectively. 
Figure 23: Average neuroantigen-specific suppressive ability is deficient during acute MS exacerbation

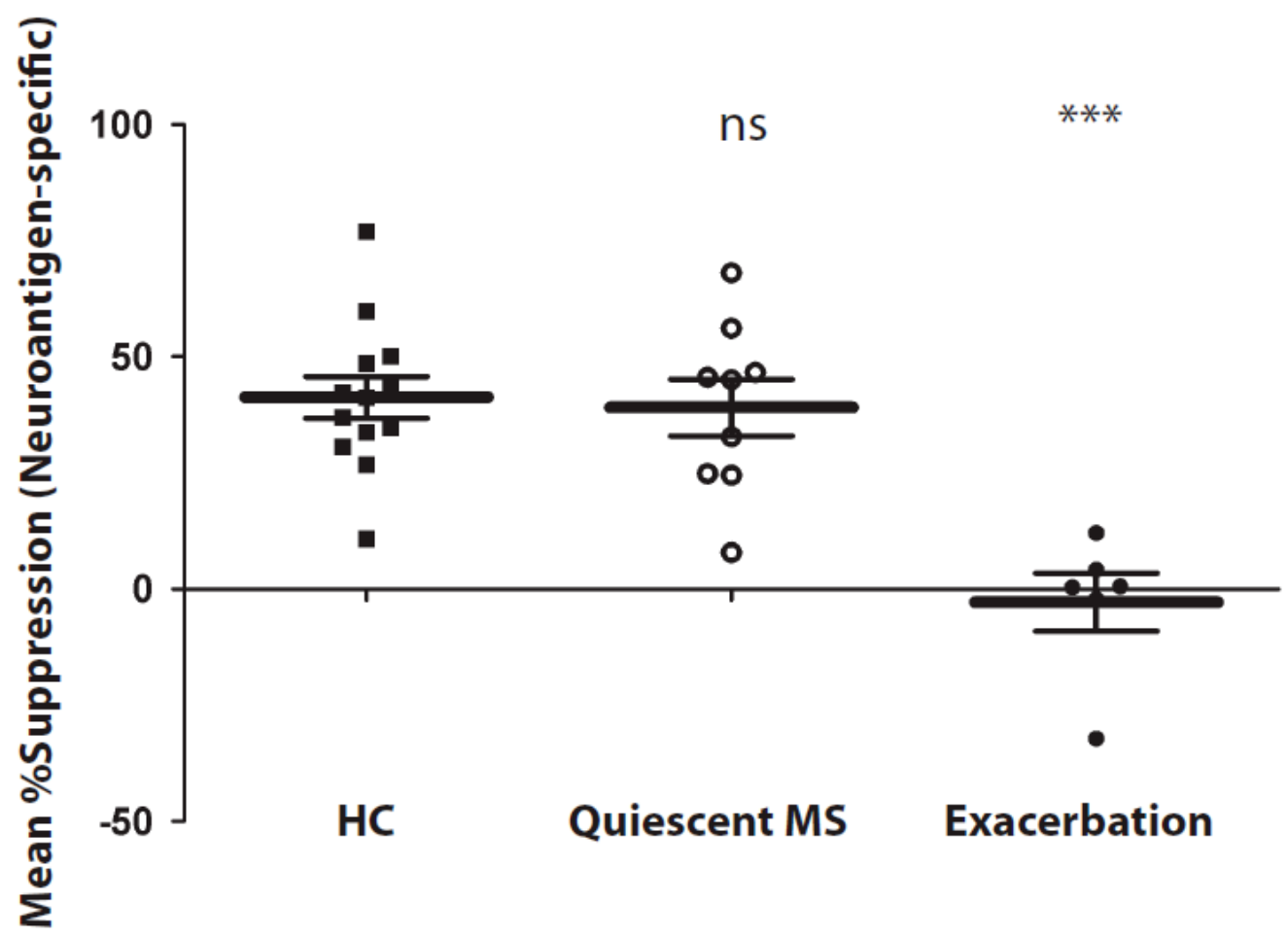

Data from Figure 22 were re-evaluated to obtain a single "mean neuroantigenspecific suppression" per subject, by averaging the \% suppression against various neuroantigens. Each dot represents the mean neuroantigen-specific CD8 suppression per subject in HC, quiescent MS patients and acute MS exacerbation patients, as indicated (ns=not significant, compared to HC; $* * *=p<0.001$, compared to either $\mathrm{HC}$ or quiescent MS). $\mathrm{P}$ values $>0.05$ were not significant with "ns" notated where applied in figures. Likewise $\mathrm{P}$ values $<0.001$ were significant with “***” notated respectively. 
Figure 24: Global CD8+ $\mathrm{T}$ cell suppressive ability is deficient during acute MS exacerbation

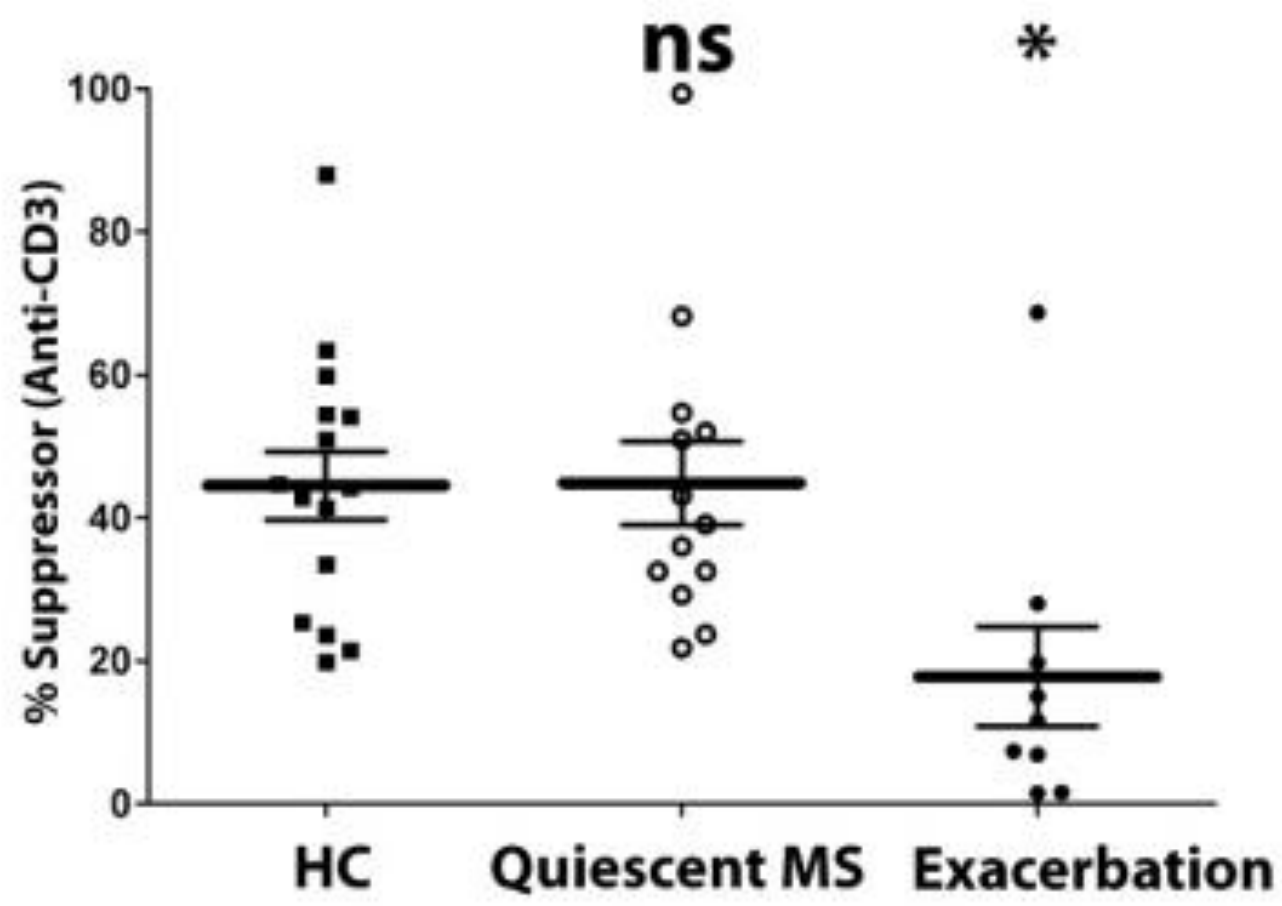

Panels show cumulative \% suppression data at the 1:0.25 responder:suppressor ratio from assays containing anti-CD3. $P$ values $>0.05$ were not significant with "ns" notated where applied in figures. Likewise P values 0.01 to 0.05 were significant with "*” notated respectively. 
Figure 25: Foreign-specific suppressive ability is unchanged during acute MS exacerbation

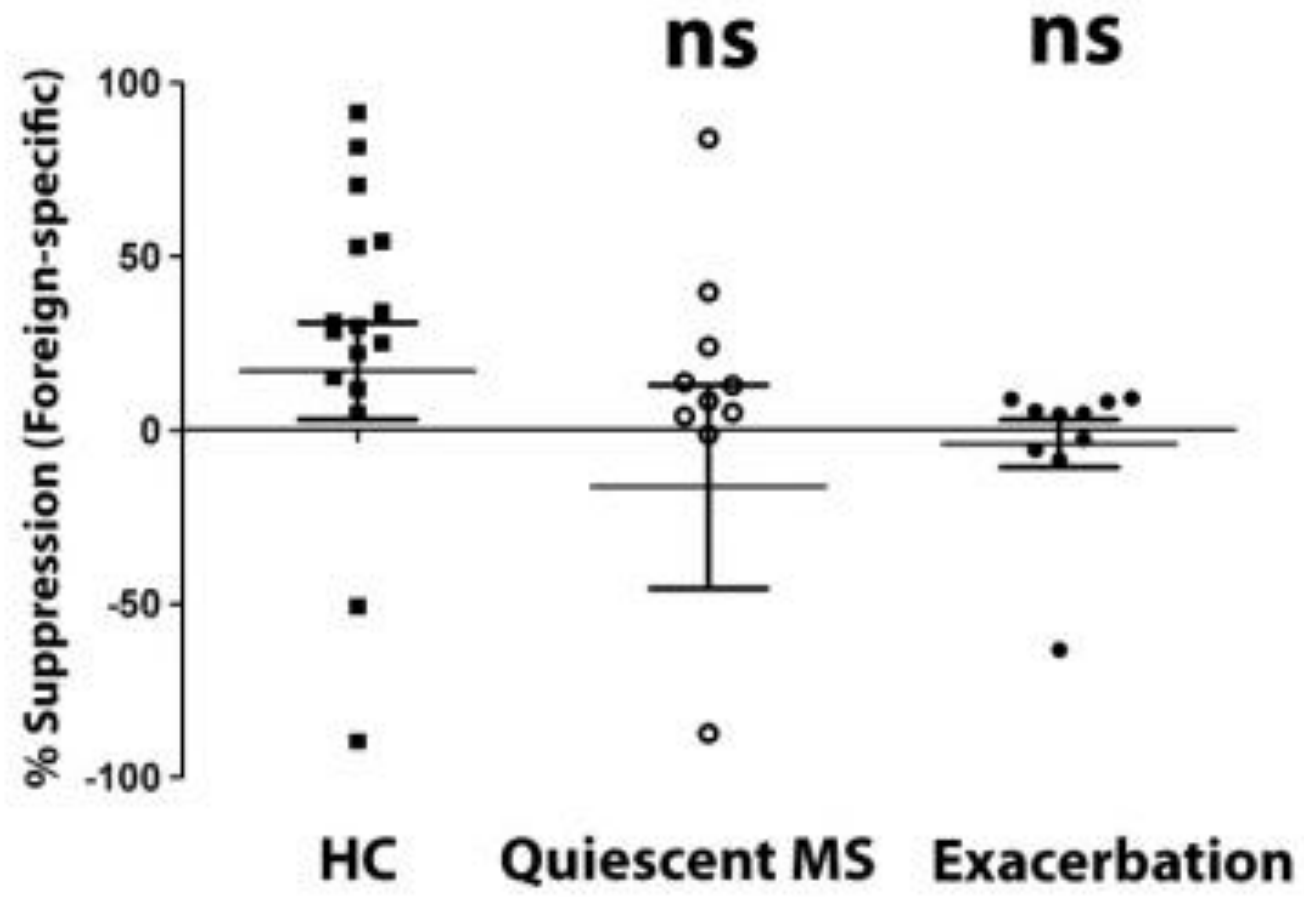

Panels show cumulative \% suppression data at the 1:0.25 responder:suppressor ratio from assays containing foreign antigens. $\mathrm{P}$ values $>0.05$ were not significant with "ns" notated where applied in figures. 
To address whether the lack of suppression could be explained by major changes in T-cell subsets, I first evaluated CD4:CD8 ratios across various cohorts and found no significant differences between any of the cohorts, especially between quiescent MS $(1.75 \pm 0.69)$ vs. acute exacerbation $(2.12 \pm 0.72)$. I further evaluated whether there may be an absence of CNS-specific CD8 reactivity in the peripheral blood during acute exacerbation or enhanced activation or proliferation of CNS-specific CD8 cells in the suppression assays. Using CMTPX as a cell tracker, I was able to specifically evaluate CD8 T-cell activation. While CMTPX is not optimal for use as a proliferation dye, I could evaluate total CD25 expression by the CMTPX-stained CD8 cells in these cultures. I found that, albeit slightly diminished, CNS-specific CD8 reactivity was detectable even during acute exacerbation (Figure 26, Figure 27, Figure 28), suggesting that these responses may be functionally different rather than simply quantitatively suppressed. 
Figure 26: During suppression assays, MS and healthy controls share similar CD8+ $T$ cell activation to neuroantigen

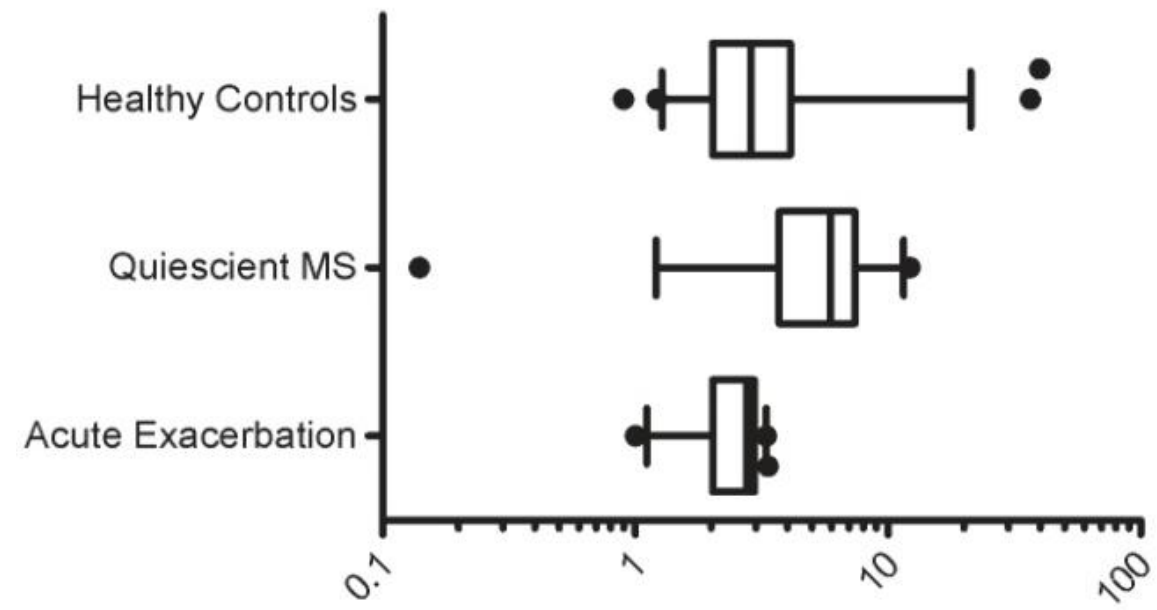

Neuroantigen-Specific CD8 Response (CD25+)

CMTPX-stained CD8+ $\mathrm{T}$ cells from suppression assays were evaluated for activation in the presence of neuroantigens by comparing their CD25 expression to that in the absence of antigen. Cumulative stimulation indices (percent CD25 with stimulus divided by percent CD25 with no stimulus) are shown from 50 neuroantigenic responses from 15 healthy controls, 29 responses from 11 quiescent MS patients, and 47 responses from 9 acute exacerbation patients. P values $>0.05$ were not significant with "ns" notated where applied in figures. 
Figure 27: During suppression assays, MS and healthy controls share similar CD8+ $T$ cell activation to foreign antigen

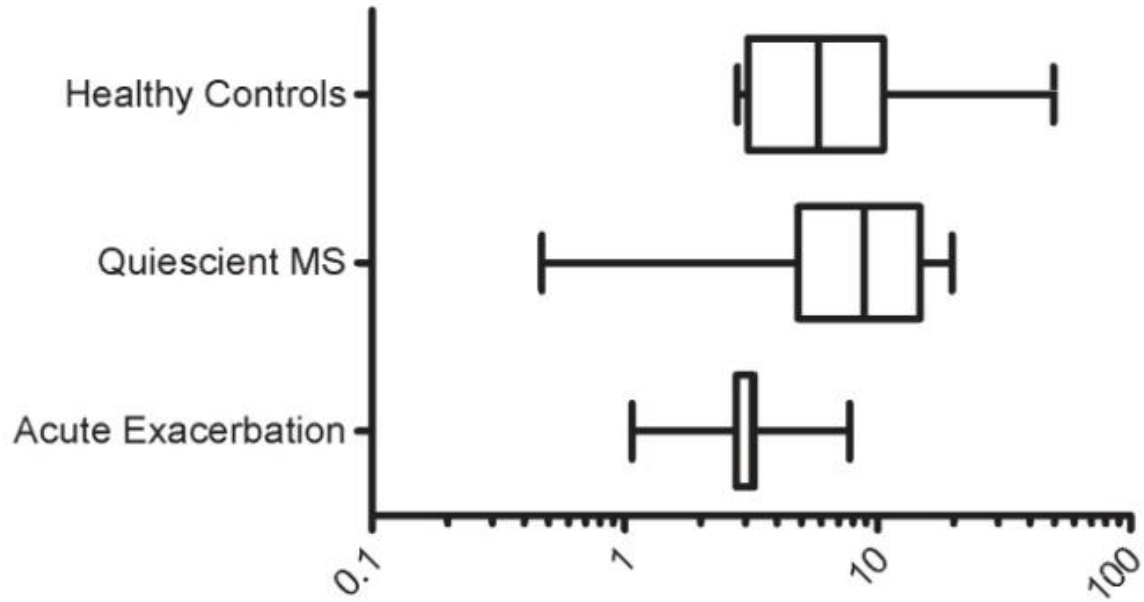

Foreign Antigen-specific CD8 Response (CD25+)

CMTPX-stained CD8+ $\mathrm{T}$ cells from suppression assays were evaluated for activation in the presence of foreign antigen (CMV, TT) by comparing their CD25 expression to that in the absence of antigen. Cumulative stimulation indices (percent CD25 with stimulus divided by percent CD25 with no stimulus) are shown from 17 foreign antigen responses from 15 healthy controls, 13 responses from 11 quiescent MS patients, and 11 responses from 9 acute exacerbation patients. P values $>0.05$ were not significant with "ns" notated where applied in figures. 
Figure 28: During suppression assays, MS and healthy controls share similar CD8+ $T$ cell activation to anti-CD3

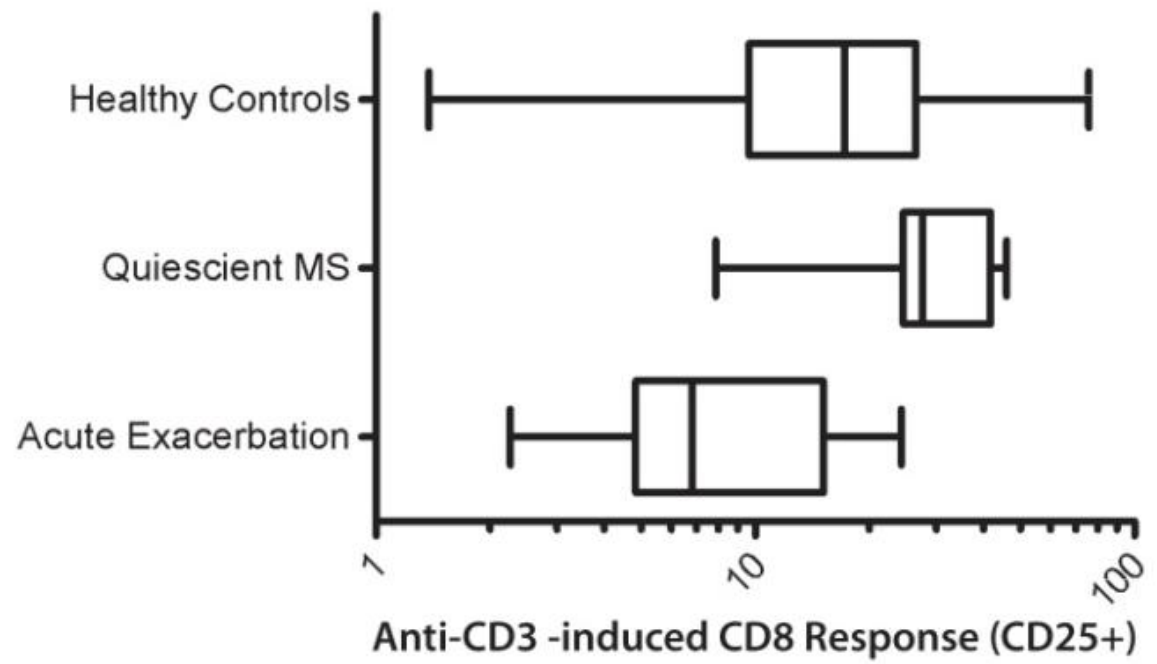

CMTPX-stained CD8+ $\mathrm{T}$ cells from suppression assays were evaluated for activation in the presence of anti-CD3 by comparing their CD25 expression to that in the absence of anti-CD3. Cumulative stimulation indices (percent CD25 with stimulus divided by percent CD25 with no stimulus) are shown from 15 antiCD3 responses from 15 healthy controls, 11 responses from 11 quiescent MS patients, and 9 responses from 9 acute MS exacerbation patients. $\mathrm{P}$ values $>0.05$ were not significant with "ns" notated where applied in figures. 
NEUROANTIGEN-SPECIFIC CD8+ T CELL SUPPRESSIVE ABILITY CORRELATES WITH DAYS SINCE LAST RELAPSE

To gauge the clinical relevance of these findings further, I asked whether there was any correlation between CD8 suppressor ability and the distance from an acute clinical episode. I found that the duration from the latest clinical episode correlated significantly $(\mathrm{p}=0.0398$ ) with CNS-specific CD8 suppression (Figure 29 ), but not with foreign-specific $(\mathrm{p}=0.9574)$ (Figure 30) or global (anti-CD3mediated) suppression ( $\mathrm{p}=0.4373$ ) (Figure 31). In contrast to foreign-specific and anti-CD3-induced CD8 suppression, most of the neuroantigen-specific CD8+ suppressive function, plotted versus time since last relapse, could be explained by the regression line demonstrated in Figure 29. This suggested that correction of the CNS-specific CD8 suppressor deficit would correlate with recovery from an acute relapse. 
Figure 29: Neuroantigen-specific CD8+ T-cell suppressive-ability correlates with days since last relapse

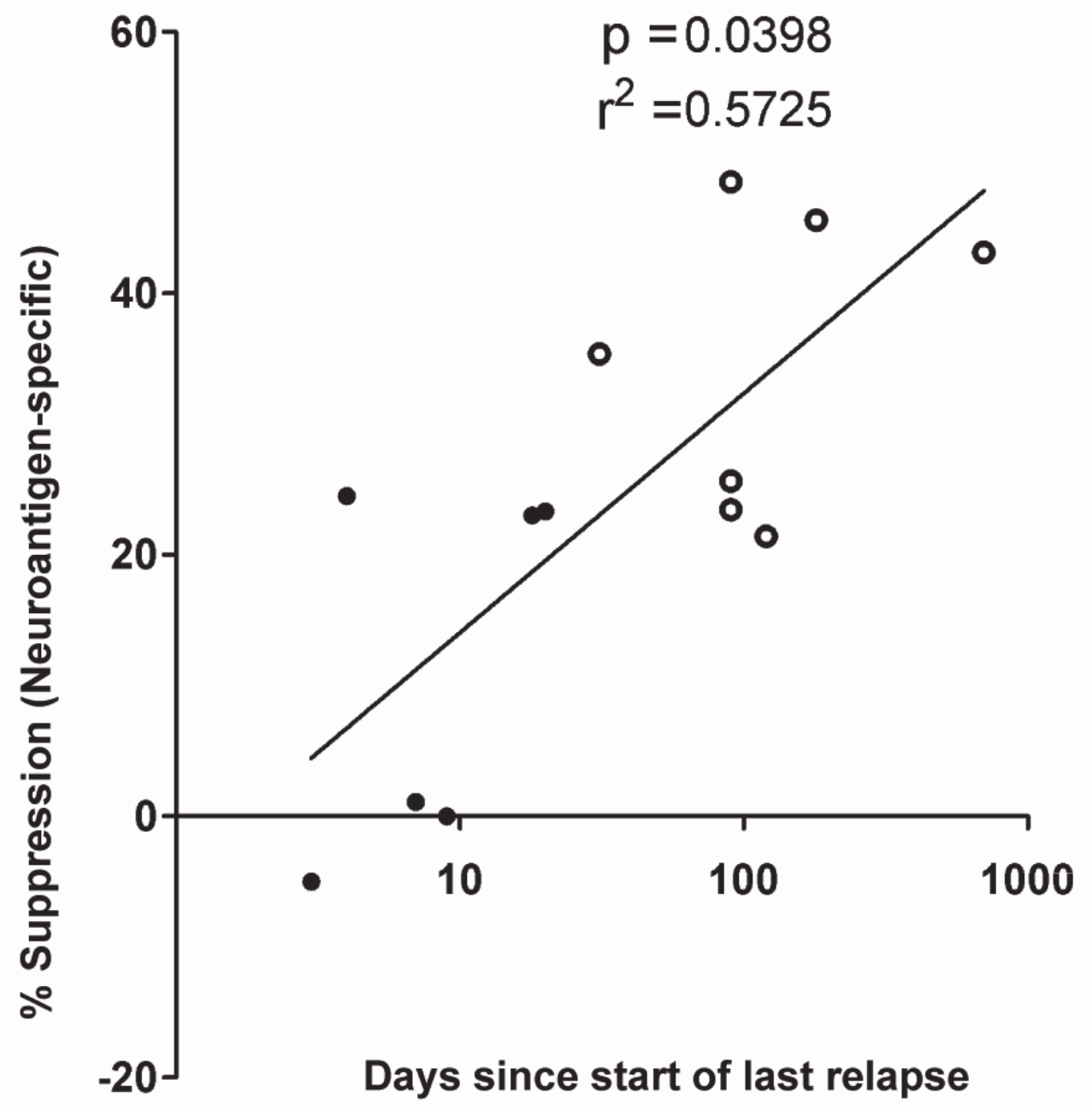

Each dot represents one of $13 \mathrm{MS}$ patients. Dots represent average CD8+ T-cell suppressive ability of individual MS patients in the presence of neuroantigens. Closed and open circles are acute MS exacerbation and quiescent MS patients, respectively. $\mathrm{R}$ squared values are shown for nonlinear regression assuming a semi-log X line model. P values are shown for correlation analysis. 
Figure 30: Foreign antigen-specific CD8+ T-cell suppressive-ability does not correlate with days since last relapse

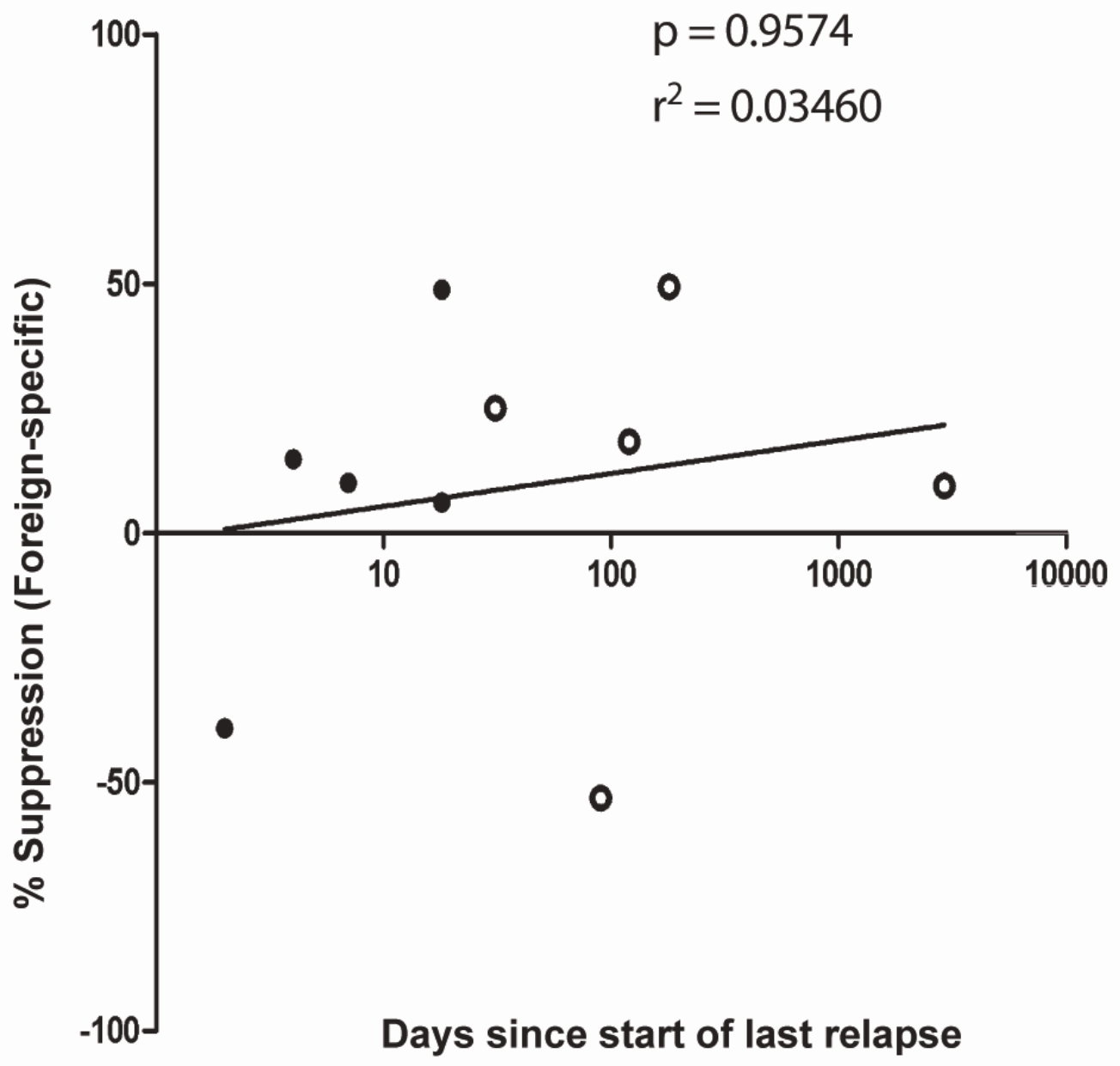

Dots represent average CD8+ T-cell suppressive ability of individual MS patients in the presence of foreign antigens. Closed and open circles are acute MS exacerbation and quiescent MS patients, respectively. $\mathrm{R}$ squared values are shown for nonlinear regression assuming a semi-log $\mathrm{X}$ line model. $\mathrm{P}$ values are shown for correlation analysis. 
Figure 31: Global CD8+ T-cell suppressive-ability does not strongly correlate with days since last relapse

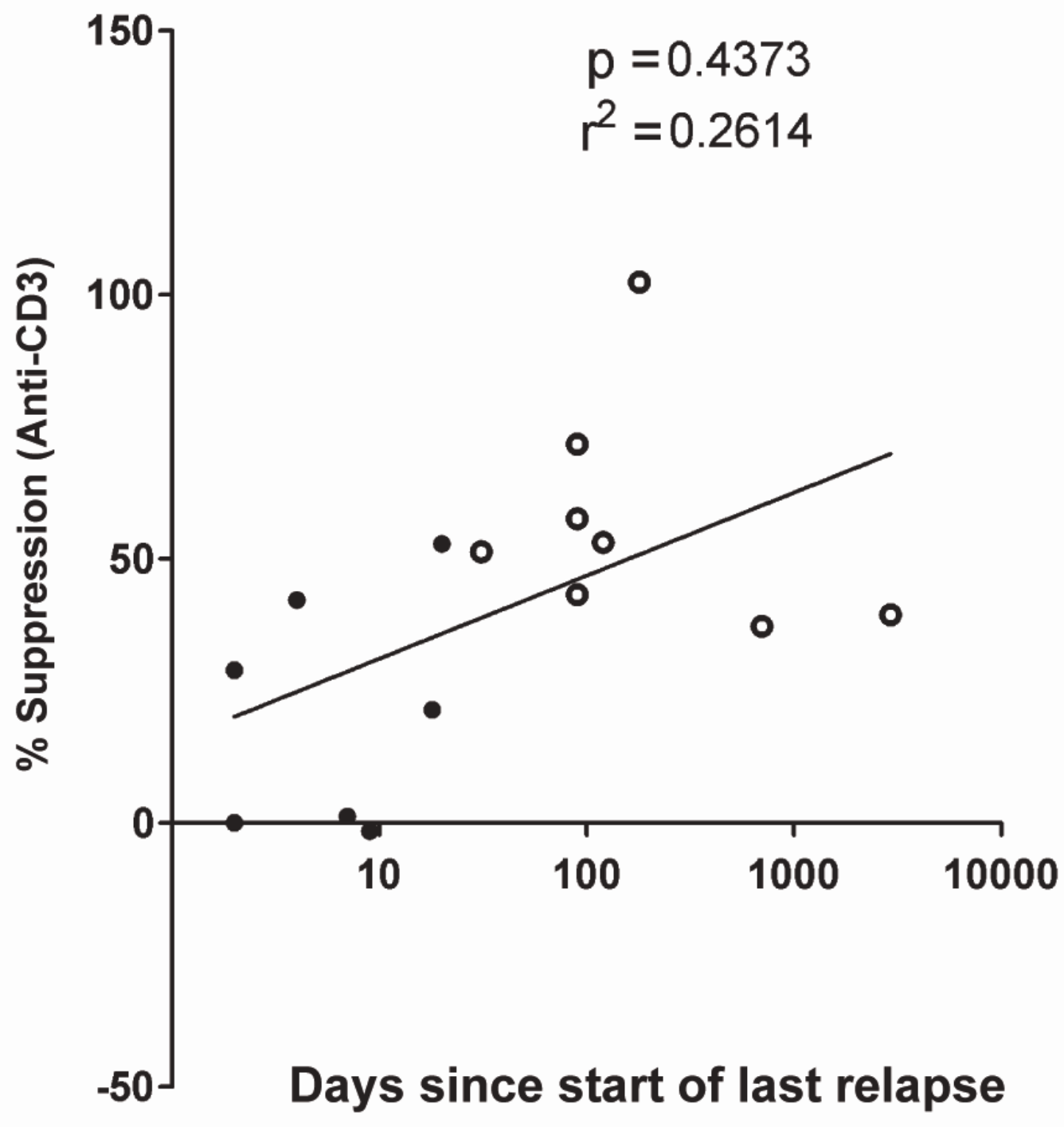

Dots represent average CD8+ T-cell suppressive ability of individual MS patients in the presence anti-CD3. Closed and open circles are acute MS exacerbation and quiescent MS patients, respectively. R squared values are shown for nonlinear regression assuming a semi-log $\mathrm{X}$ line model. $\mathrm{P}$ values are shown for correlation analysis. 
NEUROANTIGEN-SPECIFIC CD8+ T CELL SUPPRESSIVE ABILITY CORRELATES WITH DAYS SINCE LAST RELAPSE AND RECOVERY

To test this hypothesis prospectively, I re-evaluated a subset of the subjects longitudinally, after their disease had become clinically quiescent either with or without immunomodulatory therapy (Figure 32, Figure 33). We observed a robust and significant recovery of the CNS-specific CD8 suppressive ability, whether viewed as suppression against multiple neuroantigens (Figure 32) or as mean suppression per subject (Figure 33). Again, foreign-specific CD8 suppression showed no changes over time (Figure 34), whereas there was some recovery of anti-CD3-based suppression (Figure 35). In one MS patient, I was able to perform the CNS-specific suppression assay before an exacerbation, shortly afterwards, and once again afterwards later (Figure 36). Compared to before the exacerbation, I observed an increase in the number of CNS-specific CD4+ $\mathrm{T}$ cell responses shortly after the exacerbation, represented by the number of data points at day -84 and +18 , respectively. The number of CNS-specific CD4+ $\mathrm{T}$ cell responses in this one patient remained relative constant shortly after the exacerbation compared to approximately three months later, represented by the number of data points at day +18 and +118 , respectively. Interestingly, the ability of the CD8+ T cells to suppress CNS-specific CD4+ T cell responses was marked improved 118 days after the exacerbation compared to the only 18 days after the exacerbation. This suggested that the immunological changes taking place during an exacerbation involve both a transient decrease in the CD8+ T cells to suppress neuroantigen-specific CD4+ $\mathrm{T}$ cells responses and a transient 
increase in numbers of CNS-specific CD4+ $\mathrm{T}$ cells and/or their resistance to suppressive ability of CD8+ T cells. 
Figure 32: Neuroantigen-specific CD8+ T-cell suppressive-ability correlates with days since last relapse and recovery

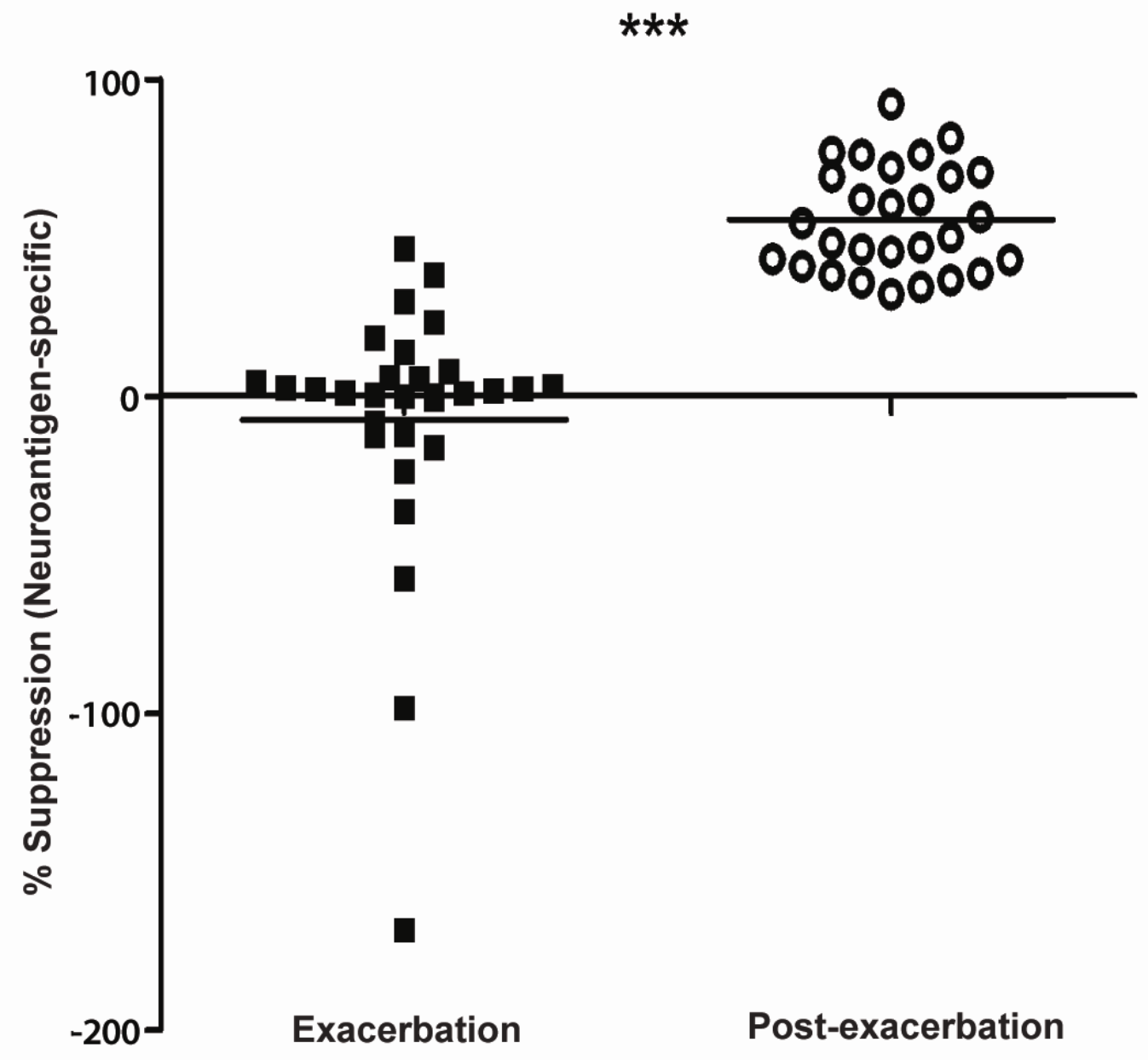

Dots represent neuroantigen-specific suppression assays performed longitudinally during exacerbation and after a quiescent clinical state as reached. Closed squares and open circles represent patients who averaged 12 and 81 days since start of last relapse, respectively. P values $<0.001$ were significant with “****” notated. 
Figure 33: Mean neuroantigen-specific CD8+ T-cell suppression decreases transiently during acute MS exacerbation

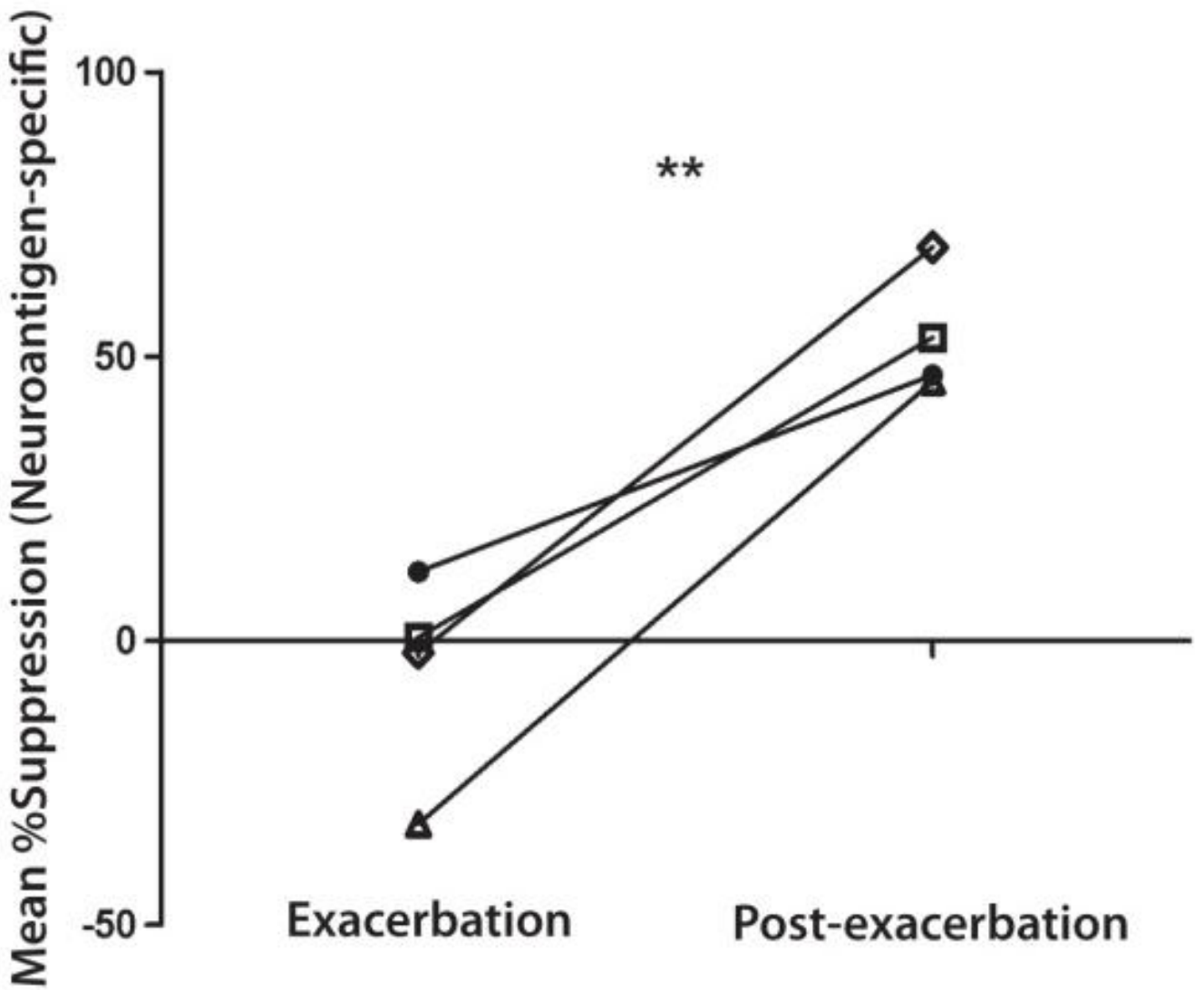

Data from Figure 32 were re-evaluated to obtain a single "mean neuroantigenspecific suppression" per subject, by averaging the \% suppression against various neuroantigens. Each dot represents the mean neuroantigen-specific CD8 suppression per patient during an exacerbation and in a longitudinal specimen collected after quiescent state was established either with or without immunomodulatory therapy. Dot shape and lines indicate paired longitudinal values $(* * \mathrm{p}<0.01)$. At follow-up, the four patients averaged 81.3 days since start of last relapse. At post-exacerbation, one patient was treatment naïve through out (closed circle), one was on Copaxone for 3 months (open triangle), one was on IFN-beta (open square) and one stopped IFN-beta therapy after two doses (open diamond). $\mathrm{P}$ values 0.001 to 0.01 were significant with “**” notated respectively. 
Figure 34: Foreign antigen-specific CD8+ T-cell suppressive-ability does not correlate with days since last relapse and recovery

ns

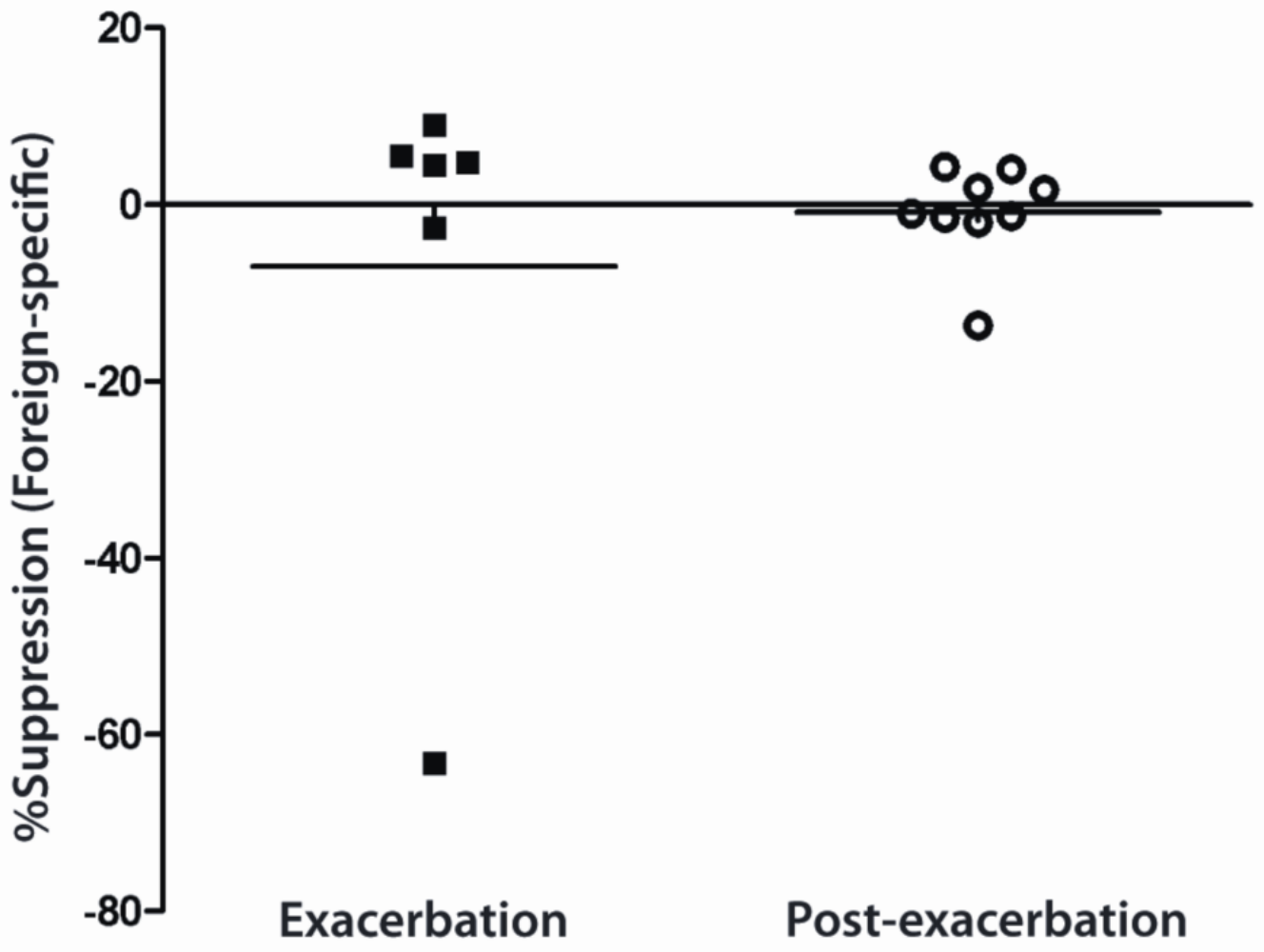

Foreign-specific CD8 suppression from four acute MS exacerbation patients during and post-exacerbation is shown. $\mathrm{P}$ values $>0.05$ were not significant with "ns" notated where applied in figures. 
Figure 35: Global CD8+ T-cell suppressive-ability does not correlate with days since last relapse and recovery

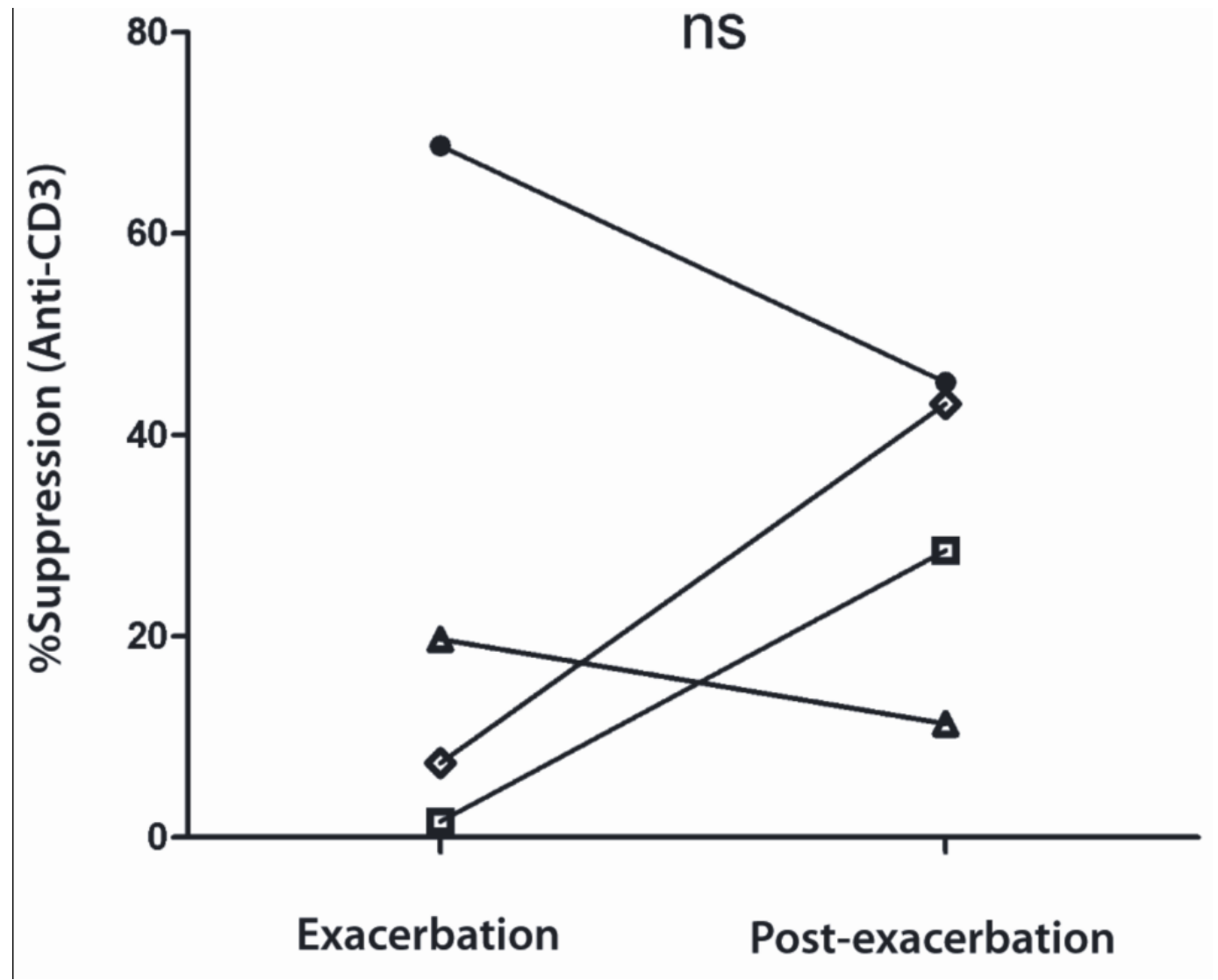

Longitudinal anti-CD3-stimulated CD8+ T cell suppressive ability is depicted.

Each dot represents the mean global CD8 suppression per patient during an exacerbation and in a longitudinal specimen collected after quiescent state was established either with or without immunomodulatory therapy. At follow-up, the four patients averaged 81.3 days since start of last relapse. At post-exacerbation, one patient was treatment naïve through out (closed circle), one was on Copaxone for 3 months (open triangle), one was on IFN-beta (open square) and one stopped IFN-beta therapy after two doses (open diamond). $\mathrm{P}$ values $>0.05$ were not significant with "ns" notated where applied in figures. 
Figure 36: Neuroantigen-specific CD8+ T-cell suppression decreases transiently during acute MS exacerbation

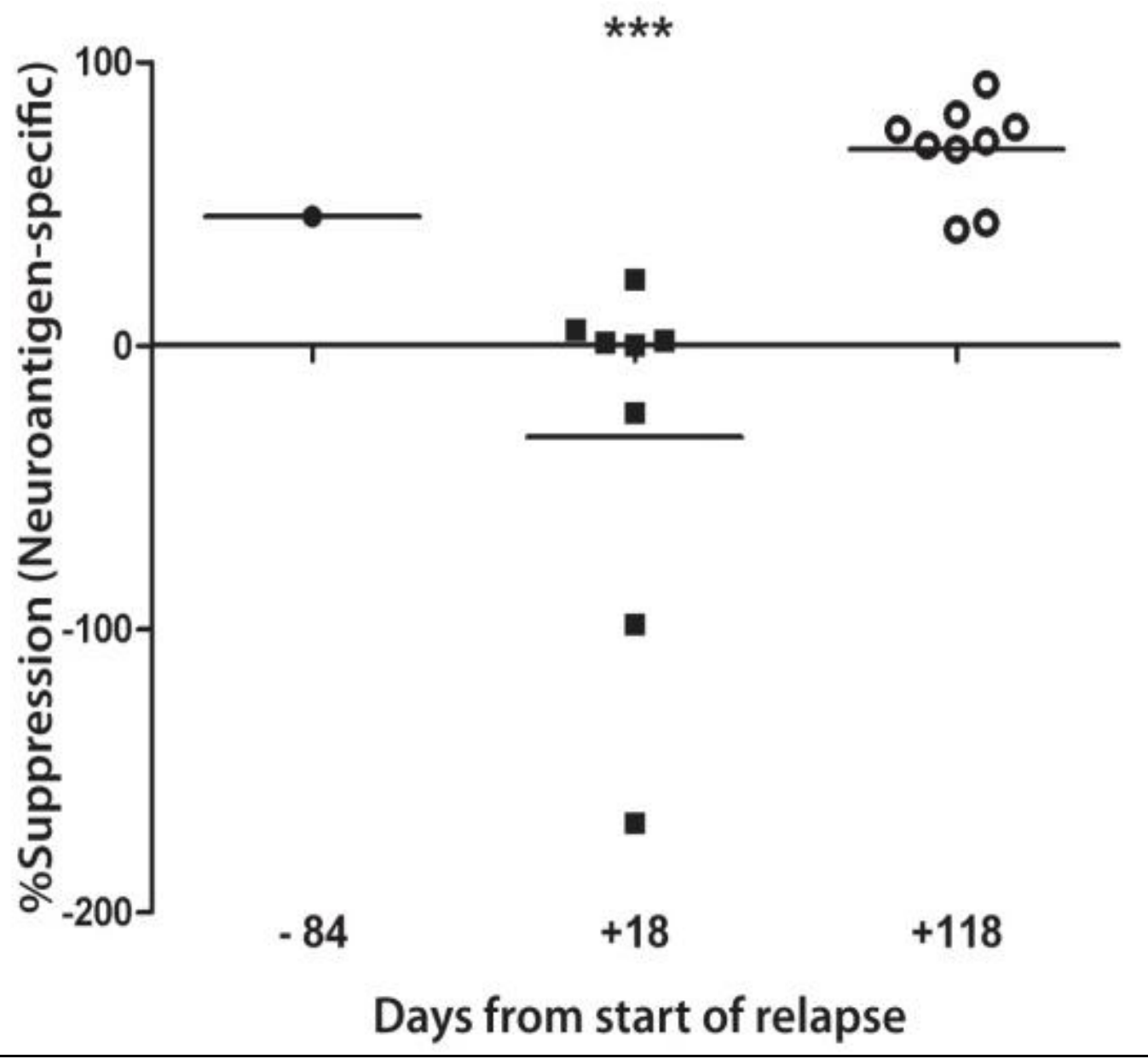

These data represent neuroantigen-specific suppression assays performed longitudinally from a single MS patient, who was evaluated at a quiescent stage of disease before and after an acute exacerbation. $\mathrm{P}$ values $<0.001$ were significant with “***" notated, comparing +18 to +118 days post-exacerbation. 


\section{NEUROANTIGEN-SPECIFIC CD8 SUPPRESSION REQUIRES HLA CLASS I} AND INTERFERON GAMMA

To test this hypothesis, I conducted CNS-specific suppression assay with the addition of blocking antibodies (Figure 37). I observed a decrease in neuroantigen-specific suppressive ability by CD8+ T cells upon the addition of a commercially available cocktail of anti-HLA (classical) class I antibodies. This suggested that at least some suppressive CNS-specific CD8+ T cells were HLA class I (A, B, or C) restricted. I then repeated similar experiments with additional blocking antibodies (Figure 38). I observed a large decrease in neuroantigenspecific suppressive ability by CD8+ T cells upon the addition of anti-IFN $\gamma$ or anti-HLA-E antibodies. I observed a smaller, yet significant, decrease upon the addition of anti-PD-1, anti-NKG2D, and anti-IL10 antibodies. This suggested that neuroantigen-specific suppression by $\mathrm{CD} 8+\mathrm{T}$ cells may involve both classical and non-classical antigen-presentation pathways. The suppressive mechanism of CNS-specific CD8 $+\mathrm{T}$ cells may involve both contact-dependent and -independent processes. 
Figure 37: CNS-Specific CD8+ T cells require HLA class I, not TNF, in order to suppress CNS-specific CD4+ T cells

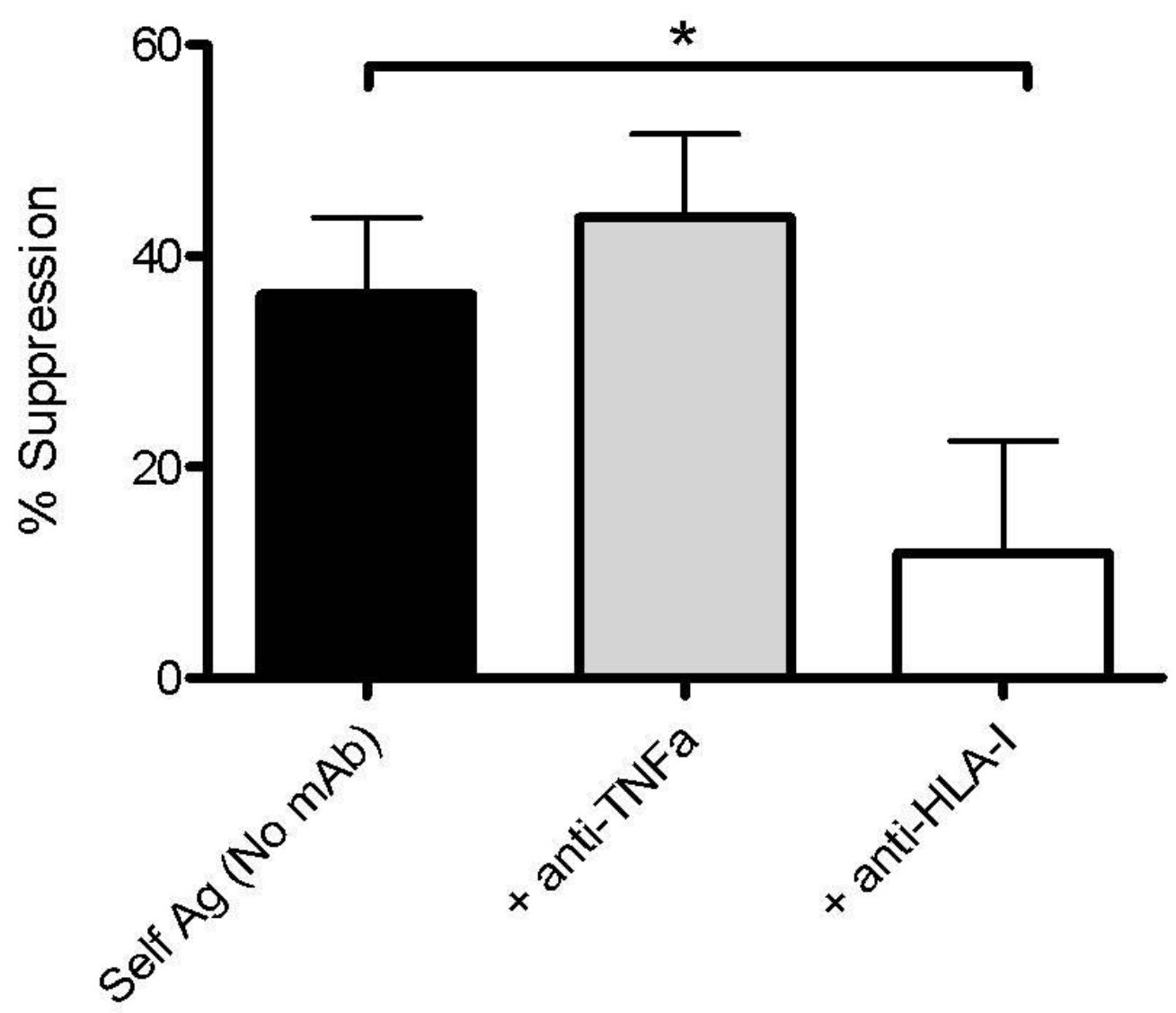

Ex vivo PBMC were used in myelin self antigen-specific flow-based suppression assays +/- anti-TNF or -HLA class I antibodies. P values 0.01 to 0.05 were significant with "*” notated respectively. 
Figure 38: Neuroantigen-specific suppression by CD8+ $\mathrm{T}$ cells was dependent upon HLA class I, IFN $\gamma$, with possible partial involvement by NKG2D, PD-1, and IL10

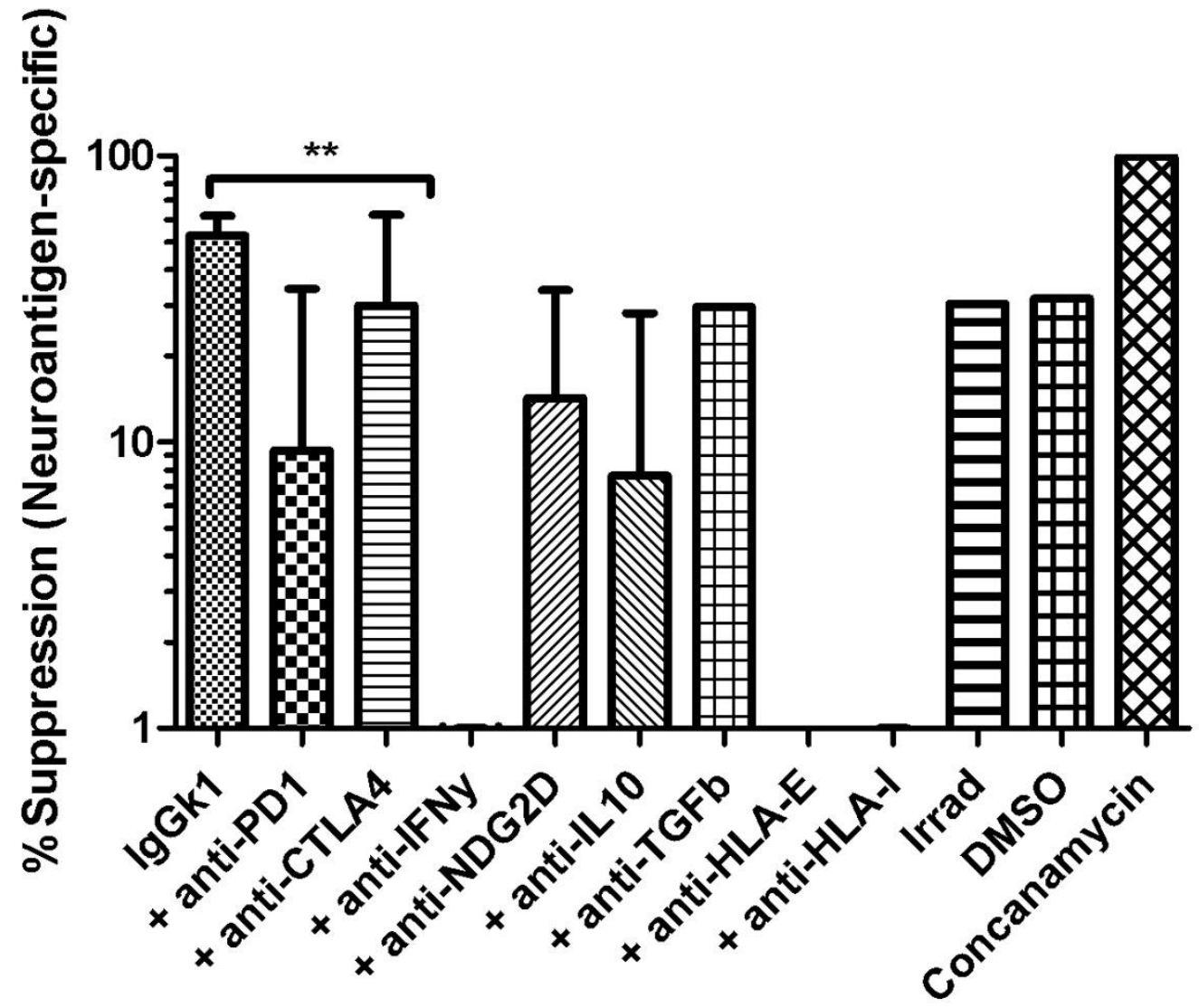

Ex vivo PBMC were used in myelin self antigen-specific flow-based suppression assays in the presence of antibodies or CD8+ T cells were exposed to physical and chemical stimuli prior to suppression assay. $\mathrm{P}$ values 0.001 to 0.01 were significant with “**” notated respectively. 


\section{ANTIGEN-SPECIFIC SUPPRESSION IS HARBORED IN THE CD8+CD62L-}

\section{SUBSET}

To test this hypothesis, I conducted a flow-based suppression assay using bulk CD8+ $\mathrm{T}$ cells from healthy subjects and their magnetically sorted CD62L subsets as suppressor cells (Figure 39, Figure 40). I observed in suppression assays, using neuroantigen and Copaxone as the stimulus, that the CD8+CD62Lsubsets possessed increased mean suppressive ability compared to bulk CD8+ T cell and the CD8+CD62L+ subset. This suggested that CNS- and GA-specific suppressive ability by $\mathrm{CD} 8+\mathrm{T}$ cells may share similar mechanisms and that CD8+ cells having the most suppressive ability may include terminally differentiated or exhausted CD8+ T cells. 
Figure 39: CNS-specific suppression is harbored in the CD8+CD62L(-) subset

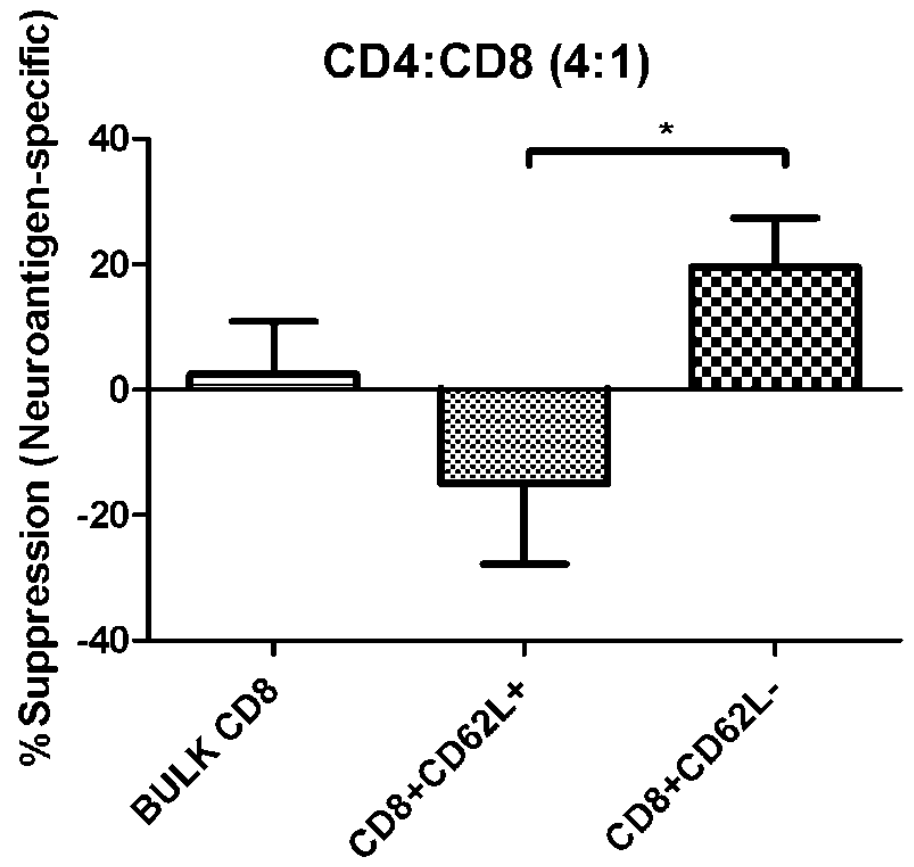

Representative of flow-based suppression assays from 5 healthy controls. P values 0.01 to 0.05 were significant with “*” notated respectively. 
Figure 40: GA-specific suppression is harbored in the CD8+CD62L(-) subset

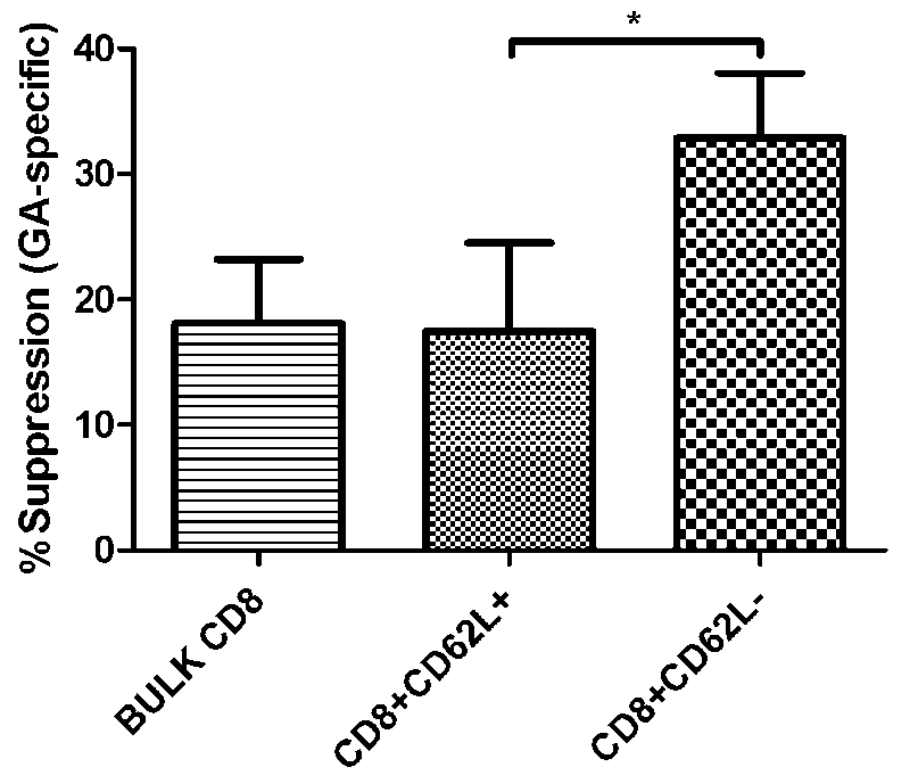

Representative of flow-based suppression assays from 2 healthy controls. P values 0.01 to 0.05 were significant with “*” notated respectively. 
GLOBAL SUPPRESSIVE ABILITY IN ADULTS IS HARBORED IN THE CD8+CD28+ SUBSET

To test this hypothesis, I conducted a flow-based suppression assay using bulk CD8+ T cells and their magnetically sorted CD28 subsets, taken from both adult PBMCs and neonatal cord blood, as suppressor cells (Figure 41). I observed in suppression assays, using anti-CD3 as the stimulus, that the CD8+CD28+ subsets from adults possessed increased suppressive ability compared to CD8+CD28- cells. Contrarily, CD8+CD28- cells from cord blood possessed increased suppressive ability compared to bulk CD8+ cells from cord blood. This suggested that the cells responsible for global, non-specific, CD8+ $\mathrm{T}$ cell suppressive ability may change over time due to age-related developmental factors, antigen-exposure, and other unknown factors. 
Figure 41: Global CD8 suppressive ability is harbored in the CD28+ subset in adults, in contrast to neonates. CD8+ subset harboring non-specific suppression changes over a lifetime

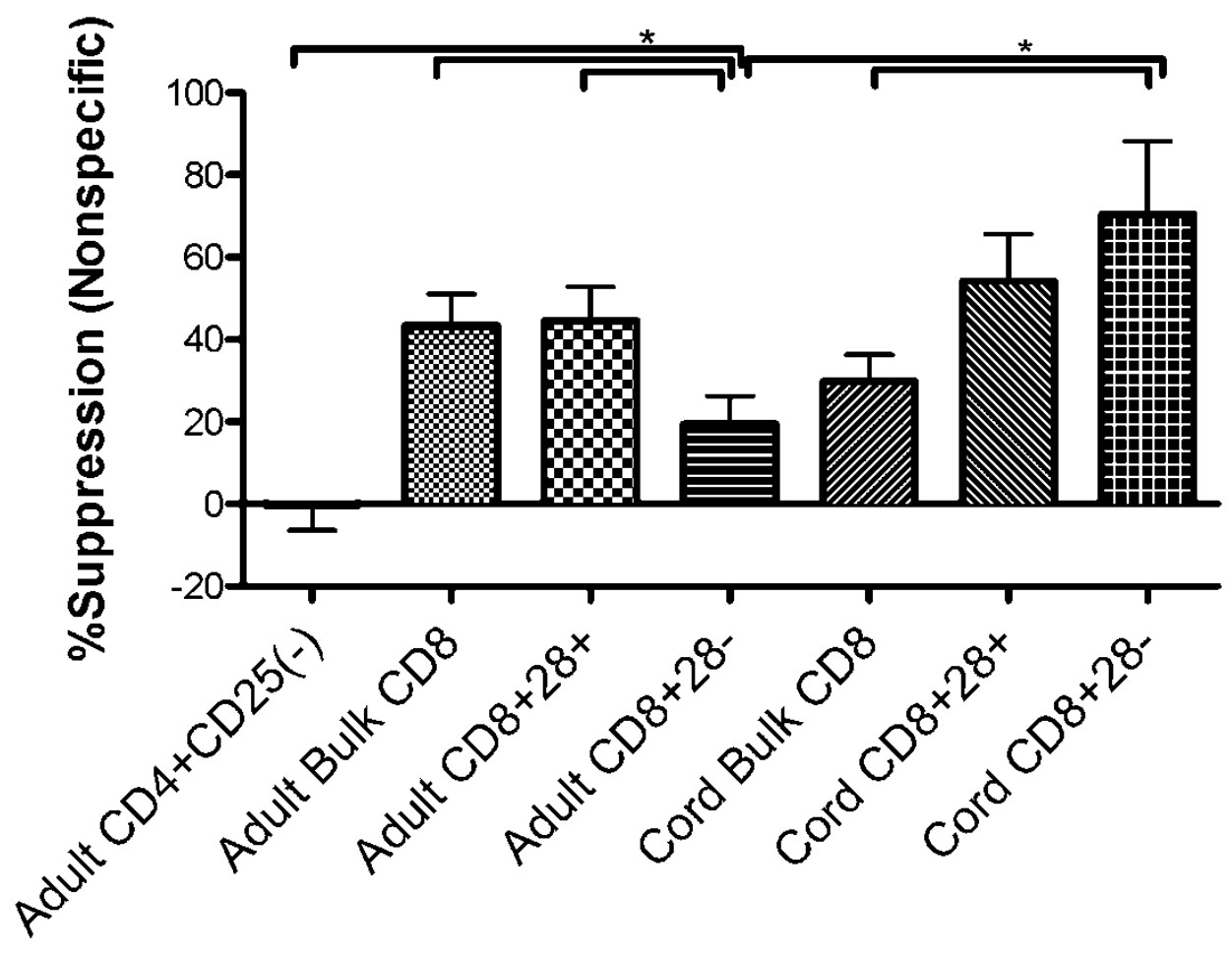

Representative of flow-based suppression assays from 9 healthy adults and 5 neonates. Ex vivo bulk CD8+ T cells and magnetically sorted subsets from adults and cord blood were put into flow-based suppression assays with autologous antiCD3 stimulated CD4+CD25(-) $\mathrm{T}$ cells for 7 days. Autologous CD4+CD25(-) T cells were used as a negative control for suppression. P values 0.01 to 0.05 were significant with "*” notated respectively. 


\section{CHAPTER 4: DISCUSSION}

\section{DEFICIENT CNS-SPECIFIC CD8 TREG IN MS ACUTE EXACERBATIONS}

To my knowledge, these studies were the first evidence that human autoreactive CNS-specific CD8+ T-cells play an immune regulatory role, in contrast to foreign-antigen-specific effectors. Moreover, my studies showed a clear clinical relevance for this regulatory role, in that suppressive activity is greatly diminished during relapses of MS and recovered as the patients enter remission. Classically, CNS-targeted, MHC Class I-restricted CD8+ T-cells were thought to have a pathogenic role in disease, with reports demonstrating in vitro cytotoxic killing of oligodendrocytes $[94,103]$. However, my studies identified an unexpected and novel immune regulatory role in both $\mathrm{HC}$ and quiescent MS patients, corroborating studies in EAE, where CNS-specific CD8+ T-cells inhibited disease, whereas control antigen-specific ones did not [306]. Sporadic, acute exacerbations are characteristic of the relapsing-remitting form of MS. While MS suppressor cell dysfunction has been recognized for decades, the role of CNS-specific CD8+ T-cells remains elusive in the context of accumulating disability, axon trans-section, and gliosis which are characteristic of secondary progressive MS [93, 309]. It appears that CNS-specific regulatory ability is directly or indirectly involved in the mechanism of MS clinical phase changes from remission to relapse. It still remains unclear whether underlying pathology

of chronic progressive MS exhibit similar deficient suppressor CD8+ T-cell activity [307].

\section{IMMUNOLOGIC EPIDEMIOLOGY}


Human studies are often hampered by the lack of implicit causation. Epidemiology is the study of disease in populations, and my study of MS patients resembled a case/control study. I reasoned that acute exacerbations of MS vary in frequency, severity, length, and type, and I sought to test if CNS-specific CD8+ T cell suppressive ability correlated with clinical phases of disease. I selected subjects based on their disease status and investigated a potential exposure that both groups (cases and controls) may have encountered. It would be faster and more cost-effective than first determining exposure in a random population and observing prospectively the occurrence of a rather rare disease, MS. I selected individuals with or without disease, MS, and then examined the exposure of all groups to an immunologic parameter, neuroantigen-specific $\mathrm{CD} 8+\mathrm{T}$ cell suppressive ability. From the ratio of exposure to un-exposure in the cases and controls, an odds ratio was determined for the points of this discussion. Odds ratios is defined as the number of cases exposed/cases un-exposed over controls exposed/controls unexposed, or in other words, the likelihood that those with disease were more likely to have been exposed. To determine the odds ratio of exposure between quiescent and acute MS, I first selected MS patients based on the time since their last acute exacerbation, measured their exposure by an in vitro assay, and defined the threshold of exposure level to suppressive ability. If one assumes that less than 5\% CNS-specific suppressive ability is the definition of the exposure, five of six MS patients during an acute exacerbation and one of nine quiescent MS patients were exposed to deficient CNS-specific suppressive ability, according to Figure 23. Thus, MS patients suffering from an acute exacerbation 
were 40 times more likely than quiescent MS patients to have been exposed to less than 5\% CNS-specific suppressive activity. Total MS patients were 10.5 times more likely than healthy persons to have been exposed to less than $10 \%$ CNS-specific ability, and quiescent MS patients were 1.5 times more likely than healthy persons to have been exposed to less than $10 \%$ CNS-specific suppressive activity. Clearly, larger odds ratios garner the most interest and statistical support. While the published title of my study was not, "Assuming a less than $5 \%$ CNS-specific suppression ability defines a risk exposure, MS patients during an acute exacerbation were 40 times more likely to be deficient than quiescent MS patients," I considered its accuracy before I quickly dismissed it based on its nonintuitive nature. I have focused on the novel observation and biology of CNSspecific CD8+ T cell biology for neglect of the most accurate interpretation of its epidemiological meaning. In other words, I have risked a slight inaccuracy in the published title in order to convey immunologic meaning, while minimizing bias.

One wonders, "how has it come to be that MS patients during an acute exacerbation were 40 times more likely to be deficient than quiescent MS patients?" The suppressor cells could be in the CNS and not in the blood, possibly explaining why I saw less suppressive ability in peripheral CD8+ T cell compartment. As mentioned in the results, to address whether the lack of suppression could be explained by major changes in T-cell subsets, I first evaluated CD4:CD8 ratios across various cohorts and found no significant differences between any of the cohorts, especially between quiescent MS (1.75 \pm $0.69)$ vs. acute exacerbation $(2.12 \pm 0.72)$. I further evaluated whether there may 
be an absence of CNS-specific CD8 reactivity in the peripheral blood during acute exacerbation or enhanced activation or proliferation of CNS-specific CD8 cells in the suppression assays. Using CMTPX as a cell tracker, I was able to specifically evaluate CD8 T-cell activation. While CMTPX is not optimal for use as a proliferation dye, I could evaluate total CD25 expression by the CMTPX-stained CD8 cells in these cultures. I found that, albeit slightly diminished ( $\mathrm{p}>0.05)$, CNS-specific CD8 reactivity was detectable even during acute exacerbation (Figure 26), suggesting that these responses may be functionally different rather than simply quantitatively suppressed. "How does one assign the clinical significance of less than 5\% CNS-specific suppression ability?" Like any experimental parameter, it is only clear how the range of healthy individuals compares to other cohorts. In my study, all normal individuals tested had an average greater than $5 \%$ reduction in neuroantigen-specific CD4+ activation and proliferation at the 1:0.25 responder:suppressor ratio when $\mathrm{CD} 8+\mathrm{T}$ cells were added to the culture for 7 days (Figure 23).

\section{LIMITATIONS AND MINIMIZING ERROR}

All case/control studies are susceptible to selection and recall bias. I have considered and sought to minimize other possible sources of systemic error within my study of MS patients. Test subjects were likely to be representative of the actual MS population in the United States. In selecting treatment naïve MS patients, the study may be skewed to an early phase of MS, representing a smaller fraction of the overall patient population. The Center for Multiple Sclerosis at University of Texas Southwestern Medical Center is an American neurology 
practice, where aggressive treatment is acceptable, leaving few untreated patients. Test subjects and their care-takers properly documented symptoms. Sampling was random in that a component of selection was unpredictable, outside of study inclusion and exclusion criteria.

Sampling bias may be perceived from the relatively small study size. However, large differences between relatively small clusters of data points allowed for a very small chance of incorrectly rejecting the null hypothesis. This suggests that the study possesses statistical power to provide the conclusions I have derived. I lacked the statistical power to make any conclusions about the differential treatments taken by the four MS patients' post-acute exacerbation follow-up and lacked clinical scores by measure of expanded disability status scale (EDSS) or Cambridge multiple sclerosis basic score (CAMBS). Prior studies have used CAMBS in IFNy and TNFa level correlation analyses to fatigue [310]. While CAMBS allows statistical analysis of the otherwise qualitative, retrospective, and descriptive, it is not prospective or predictive of outcome. In some respects, it would have been ideal if EDSS assessments could have been performed routinely on our patient pool. However, EDSS is not possible to perform routinely at a large, busy neurology practice, and it was simply not done on our cohort. EDSS has been shown to correlate with CAMBS disability. Use of the less cumbersome CAMBS may allow future statistical analysis of immunologic parameters.

\section{AUTOREGULATORY T CELLS}


In the field of immunology, scientists are only beginning to understand the role of autoreactive, regulatory ("autoregulatory") T-cells in autoimmune disease [58]. There has been some evidence that autoantigen-specific CD8+ T-cells may have immune regulatory properties in diabetes models $[68,311]$. Thus, chronic stimulation of CD8+ T-cells with low TCR avidity may induce regulatory function [311], perhaps explaining the therapeutic generation of antigen-specific, cytotoxic immune suppressor CD8+ T-cells following chronic copolymer-based therapy of MS [104, 245] (Figure 43). This may also explain the difference between the roles of foreign-antigen-specific CD8+ T-cells vs. autoreactive ones that tend to bear lower avidity TCR, presumably following thymic deletion of higher avidity responders. In contrast to Qa1/HLA-E-restricted suppressor CD8+ T-cells that recognize immune cell-derived peptides, autoregulatory CD8+ T-cells are stimulated by the same tissue antigens that are targets of destructive effector cells, thereby creating an autoregulatory tolerance loop.

\section{IMMUNE THERAPY USING CD8+ T CELLS}

This novel concept also unveils a potential strategy for immune therapy. While using autoreactive CD8+ T-cells as therapy may seem unorthodox, this is principally similar to generating autoantigen-reactive $\mathrm{CD} 4+\mathrm{CD} 25+\mathrm{FOXP} 3+\mathrm{T}_{\text {reg }} \mathrm{S}$ for adoptive immunotherapy. Other forms of autoreactive CD4+ $T_{\text {regs }}(\operatorname{Tr} 1, T h 3)$ have also shown promise in animal models. CD8+ T-cells, representing an underappreciated arm of peripheral immune tolerance, afford an attractive form of adoptive immunotherapy, especially in the context of clinical relapses. In that regard, my lab has shown recently that CNS-specific CD8+ T-cells can inhibit 
ongoing EAE [306], dependent on cytotoxic and immune modulatory mechanisms. The phenotypic characteristics of regulatory CD8+ T-cells are not definite and, depending on the model, may range from a CD28(-) [37-43], $\gamma \delta+$ [45], CD25+ [46], CD122+ [47], CD103+ [49, 50], PD-1+ [53] or FOXP3+ [54, 56, 71, 312], among others [313]. In which context neuroantigen-specific CD8+ T-cells regulate, and how, is still unclear. Our preliminary studies reveal autoregulatory CNS-specific suppressor activity in multiple such subsets, with the common features being cytokine- and contact-dependent processes (including cytotoxicity) and an absolute requirement for HLA-Class I (Figure 37, Figure 38). Detailed dissection of the characteristics and mechanisms of these cells will be an important pursuit to develop a therapeutic approach.

To summarize, our studies demonstrate a novel, clinically relevant role for neuroantigen-specific CD8+ T-cells, revealing a potential pathway of intrinsic immune regulation that may have implications for the therapy of human MS and other immune-mediated disorders.

FUNDAMENTAL DIFFERENCES BETWEEN AUTOREACTIVE AND FOREIGN SPECIFIC T CELLS

The avidity of the TCR may explain the differences in their suppressive ability. In several experiments, I attempted to isolate pure CNS- and CMVspecific T cells though stimulating CFSE-stained PBMC for 7 days and then flowsorting the CFSElow population. Using the flow-sorted cells as suppressors, I set up 7 day suppression assays with fresh autologous responders stimulated with peptide or anti-CD3. I acquired the results with the HTS on the LSR and 
analyzed the \%CD25+CFSElow of the CD4+ responders. When I used peptides (MBP or CMV) as the stimulus, the result was mostly uninterruptable as I could not effectively gate out the suppressor cells without the use of CMTPX. The antigen-specific assay lacked the sensitivity to detect suppression of any sorted populations. When I used anti-CD3 as the stimulus, interesting differences were found between the flow-sorted populations. Anti-CD3 is a monoclonal antibody that bypasses the TCR and binds directly to the CD3 signalling complex in order to polyclonally-stimulate T cells. Using anti-CD3 as the stimulus, both the MOGspecific and $\mathrm{CMV}$-specific $\mathrm{CD} 8+\mathrm{T}$ cells were able to suppress anti-CD3 stimulated CD4+ responders (data not shown). This result was unexpected as CMV-specific CD8+ T cells, in previous experiments (Figure 20, Figure 42) with peptide, had undetectable suppressive ability. I hypothesized that this might be explained by the biology of anti-CD3. The phenomenon of CNS-specific suppression by CD8+ $\mathrm{T}$ cells is likely TCR-dependent. Circumventing the engagement of the TCR eliminated the CNS-specificity of CD8+ T cell suppression. Alternatively, foreign-specific suppression by CD8+ T cells may be appreciable, just undetectable by my peptide-stimulated suppression assays (Figure 20). Using flow-sorted cells, foreign-specific CD8+ T cells possessed suppressive ability that was indistinguishable quantitatively from that of CNSspecific T cells (data not shown). Perhaps lowering the amount of anti-CD3 may reveal differences between the sorted, antigen-specific populations. Additional experiments are required to resolve these questions. In summary, my conclusions 
are that CNS-specific suppressive ability requires engagement of the TCR; antiCD3 activated CD8+ T cells suppress, regardless of antigen-specificity.

The use of anti-CD3 in suppression assays with sorted-antigen specific $\mathrm{T}$ cell lines must be repeated with proper controls before any conclusions can be made. It remains unclear if $\mathrm{CMV}$-specific $\mathrm{CD} 8+\mathrm{T}$ cells really prevent suppression or if the suppression assays lack the sensitivity to measure it. The anti-CD3 stimulated global suppression assays are thought to polyclonally activate CD8+ suppressor cells, which mediate suppression through soluble factors. Other studies have suggested that TNF $\alpha$ is required for the induction of regulatory CD8+ T cells, and that suppression occurs through an IL10-dependent mechanism. 
Figure 42: Activated neuroantigen-specific CD8+ T-cells suppress CD4+ Tcells

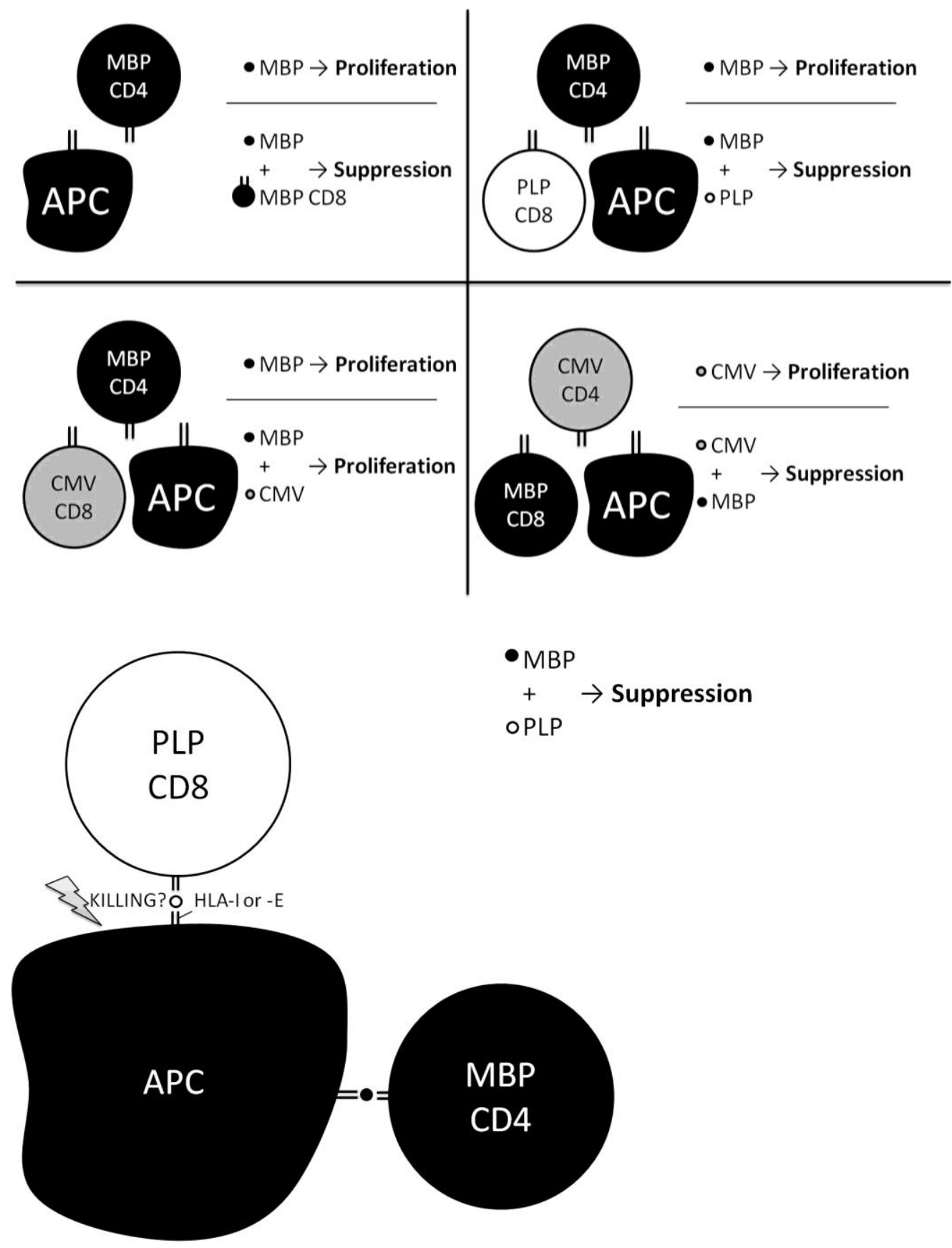

Proposed model illustration based on Figure 20. Responder CD4+ T-cell lines were cultured with APC and indicated antigens in the presence or absence of the indicated CD8+ T-cell lines. 


\section{PATHOGENIC ROLE FOR INFILTRATING CD8+ T CELLS IN MS}

Although subsets CD4+ T lymphocytes (Th1 and Th17) have been established as important mediators of demyelination, neuronal damage, and astrocyte/microglia activation in MS, CD8+ $\mathrm{T}$ cells have remained a subject of intense study and debate. CD8+ cells are oligoclonally expanded and in greater amount than CD4+ T cells in the MS lesion. Their close apposition to MHC class I-expressing cells could allow CD8+ $\mathrm{T}$ cells to recognize and target neurons, oligodendrocytes, astrocytes, microglia, CD4+ T cells, and other CD8+ T cells. The mechanisms dictating the acquisition by $\mathrm{CD} 8+\mathrm{T}$ cells of a pathogenic phenotype/function are poorly understood. During exacerbations of MS, CD8+ T cells may down-regulate inhibitory NK cell antigen receptors (NKGR/CD94) [67]. Where other studies have observed this evidence and interpreted a pathogenic potential of $\mathrm{CD} 8+\mathrm{T}$ cells [258], others have interpreted that a regulatory CD8+ $\mathrm{T}$ cell has become dysfunctional [67]. Both interpretations are correct, insofar that all studies would conclude that dysfunctional regulatory cells are pathogenic, and pathogenic cells are deregulated.

Many human studies have documented the cytotoxic ability of CD8+ T cells from MS to target each many cells types in vitro. Most human studies stress the potential of $\mathrm{CD} 8+\mathrm{T}$ cells to target either neurons, oligodendrocytes, and microglia or CD4+ $\mathrm{T}$ cells and other $\mathrm{CD} 8+\mathrm{T}$ cells, the balance being the determining facter whether to call all $\mathrm{CD} 8+\mathrm{T}$ cells in $\mathrm{MS}$ as regulatory or pathogenic. Many human studies have demonstrated the proximity of CD8+ T cells and CNS target cells in MS lesions. In human studies, associations, not 
causations, are the strongest conclusions, as described earlier in the discussion of epidemiology. Based on numerous studies upon regulatory CD8+ $\mathrm{T}$ cells, their apparent heterogeneity may serve as an example of a greater heterogeneity within bulk CD8+ T cells. Naïve, memory, effector, or exhausted CD8+ T cells may display certain aspects of a regulatory CD8+ $\mathrm{T}$ cells transiently. If CD8+ $\mathrm{T}$ cell differentiation shows any similarity to that of CD4+ T cell biology, one would expect dynamic and plastic differentiation among the forms and functions of $\mathrm{CD} 8+\mathrm{T}$ cells. For now, the master regulator transcription factors for $\mathrm{CD} 8+\mathrm{T}$ cell biology remain poorly understood. The fine line between appropriate and detrimental $\mathrm{T}$ cell activation involves essential inhibitory signals provided by the cellular environment. Infiltrating CD8 $+\mathrm{T}$ cells may be the result of missed inhibitory signals or a response to missed signals by other immune cells. A combination of animal and human studies about MS, as performed in my lab, are likely the best way forward to avoid false assumptions derived from limited approaches.

MODEL

CNS-specific CD8+ T cells are able to suppress CNS-specific CD4+ T cells. While my experiments have shown that MS exacerbations are correlated with a deficiency of CNS-specific suppressive ability, this does not prove that MS is simply caused by a lack of CD8+ T cell suppression. CNS-specific suppressive ability by $\mathrm{CD} 8+\mathrm{T}$ cell is one factor among many that are strongly correlated to MS. Still, this work may present an opportunity to explore in further studies drugs or cell-based therapies that may target CD8+ $\mathrm{T}$ cell in order to potentiate 
their suppressive function. It remains unclear if a lack of suppression during exacerbations is the fault of CD8+ suppressors or CD4+ cells being unreceptive to inhibition. Future longitudinal studies may better address these questions.

CNS-specific CD8+ $\mathrm{T}$ cells are thought to be activated through the recognition of CNS peptide in the context of classical or non-classical HLA class I on APC or CD4+ T cells. CD4+ T cells may undergo membrane exchange with APC in order to acquire cross-presented antigen in the context of HLA-class I. If CD8+ T cells are activated by APC, suppression may occur through direct killing of APC or indirectly through the selection of tolerogenic APC acting on CD4+ T cells. IFN $\gamma$ may be required for the activation or induction of regulatory $\mathrm{CD} 8+\mathrm{T}$ cells. Alternatively, IFN $\gamma$ may be required for their suppressive mechanism either by acting upon APC or CD4+ T cells. APC may be activated by IFN $\gamma$ to upregulate MHC class I or express IDO. IFN $\gamma$ may deviate CD4+ T cells toward Th1 away from Th17 or Th2 differentiation. Future experiments will interrogate these and other models of CNS-specific regulatory CD8+ T cell function. 


\section{FUTURE DIRECTIONS}

While much is known about CD4+ Treg, CD8+ Treg remain understudied and poorly understood. Using adult human peripheral blood and cord blood, we made several observations regarding CD4+ and CD8+ Treg. Prior members of my lab have made the following observations, which form part of the basis of my hypothesis. I developed a flow cytometry-based suppression assay, which utilizes CMPTX, CFSE dilution, and CD25 expression (Figure 13). Upon removal of both CD8- and CD25-expressing Treg, CD4+CD25- cells from both healthy persons and those with untreated autoimmunity harbor similar autoreactive $\mathrm{T}$ cell responses to self antigens (Figure 10). When activated by cognate antigen, self antigen-specific CD8+ Treg suppress self-specific CD4+CD25- T cells (Figure 20). Foreign, control antigen-specific CD8+ Treg are incapable of suppressing control-specific CD4+CD25(-) T cells. Suppression of CD4+CD25(-) T cells by self-specific CD8+ Treg requires HLA class I, but not TNFalpha (Figure 37). The global CD8+ Treg phenotype (mitogen stimulated) possessing the most suppressive ability changes over a lifetime (Figure 41). The role of regulatory and alloantigen-specific $\mathrm{T}$ cell biology in autoimmunity and transplantation has been examined in some detail through my work and that of others from my lab. My data provide evidence that CD8+ Treg possess potent immune suppressive ability. However, the biology of autoantigen- and alloantigen-specific CD8+ Treg remains poorly studied in the context of human disease, as does the mechanisms of global CD8-mediated suppression. To that end, pre-clinical research probing the mechanistic nature of suppression is outlined here. Pending the outcome of 
these studies, insight into the role CD8+ Treg play in the settings of transplant and autoimmunity may yield ground-breaking cell-based therapies or new drug targets.

My lab and I are currently pursuing both the mechanism of CD8+ T cell regulation and its earliest detectable effects even hours after various therapeutic measures in MS. The mechanism of action of Copaxone, an immune-modulatory agent, is poorly understood. As part of my study, I evaluated a subset of the subjects longitudinally, after their disease had become clinically quiescent either with or without immunomodulatory therapy (Figure 33, Figure 35). At postexacerbation, one patient was treatment naïve through out, one was on Copaxone for 3 months, one was on IFN-beta and one stopped IFN-beta therapy after two doses. At follow-up, I observed in all patients an increase in CNS-specific, and, in three of four, an increase in global- CD8+ $\mathrm{T}$ cell suppressive ability. At follow-up, the four patients averaged 81.3 days since start of last relapse. This suggests that the first injections of GA or IFN $\beta$ may have immediate immunologic consequences on APC or $\mathrm{T}$ cells populations that set the foundations for sustained immune regulatory responses, preventing or delaying further relapses. My lab has illustrated that GA treatment induces and restores CD8+ T cell responses in MS. The question is when.

Supporting the work of others, I have observed using flow-based suppression assays that MS patients have deficient global CD4+ and CD8+ Treg function (Figure 60). One wonders when MS therapies take effect to start improving immune-regulatory parameters. In other words, do drugs used in MS 
have early effects on T cells or APCs or both? In particular, I am interested if GA immediately impacts global- or CNS-specific CD8+ T cell suppressive ability within hours of injection. Perhaps GA enhances global- or CNS-specific suppressive ability earlier or to a greater extent than IFN $\beta$ or fingolimod or others. Do some therapeutics induce transient activation-induced regulatory CD4+ $\mathrm{T}$ cells? If intrinsic CD4+ or CD8+ regulatory $\mathrm{T}$ cell function is augmented or peaked at a particular time, this could have important therapeutic implications for dosing regimes or experimental adoptive transfer protocols. Perhaps these studies will support the notion of a drug vacation after GA has made its most potent immune regulatory response in the early treatment phase.

The mechanisms of regulatory CD8+ $\mathrm{T}$ cells are poorly understood. I have observed increased regulatory $\mathrm{T}$ cell function within certain subsets and blocked suppressive activity through the addition of neutralizing antibodies (Figure 38, Figure 39). HLA-E may act as a ligand for the TCR $\alpha \beta$, and CD8+ T cells proliferate in response to self antigen-, cytomegalovirus-, Epstein-Barr virus, Listeria monocytogenes-, Mycobacterium tuberculosis-, and Salmonella enteric -derived peptides presented in the context of HLA-E [276-279]. My lab and I are interested in better understanding the role of cytotoxic molecules and nonclassical HLA class I molecules in antigen-specific CD8+ regulatory $\mathrm{T}$ cell function. Perhaps CD4+ T cell or APC antigen-processing may allow for CNS peptides to be presented in context of HLA-E, creating a potential target for cytotoxic/regulatory CD8+ T cells. If the mechanisms behind regulatory $\mathrm{T}$ cells 
can be better understood, new strategic targets for therapeutics may be uncovered in the context of allergy, asthma, transplant, and autoimmunity. 
PROPOSED MODEL OF CNS-SPECIFIC REGULATORY T CELLS DYSFUNCTION DURING ACUTE EXACERBATION OF MS

In the center of my proposed model are HLA class I-restricted, CNStargeted CD8+ T cells (Figure 43). I have presented evidence for their immune regulatory role in both $\mathrm{HC}$ and quiescent MS patients. CNS-specific regulatory ability is directly or indirectly involved in the mechanism of MS clinical phase change. During the steady-state and remission in MS, CNS-specific regulatory ability has a measurable suppressive effect toward known pathogenic cells, CNSspecific CD4+ T cells. Suppressive action may be mediated through soluble and cytotoxic factors after an initial signal received via classical and non-classical HLA class I. Based on my studies with CD8+ T cell subsets, regulatory CD8+ T cells may be largely derived from more terminally differentiated or exhausted CD8+ T cells (Figure 44). 
Figure 43: Model of how CNS-specific regulatory ability is directly or indirectly involved in the mechanism of MS clinical phase change
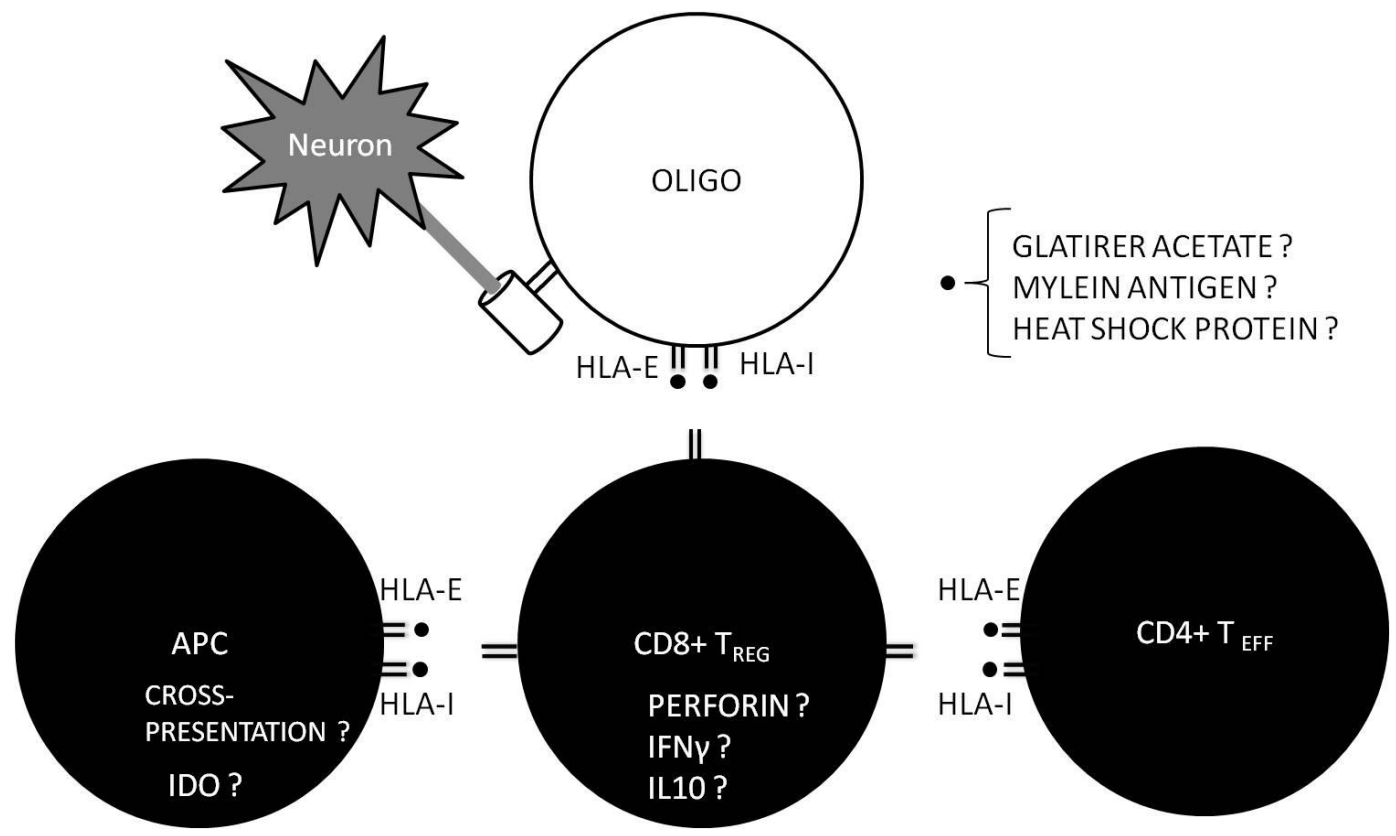

HLA class I-restricted, CNS-targeted CD8+ T cells have an immune regulatory role in both $\mathrm{HC}$ and quiescent MS patients. Acute exacerbations of MS may include decreased intrinsic suppressive ability and/or decreased CD4+T cell sensitivity to suppressor $\mathrm{T}$ cell function. 
Figure 44: CD8+ cells having the most suppressive ability may include terminally differentiated or exhausted CD8+ T cells

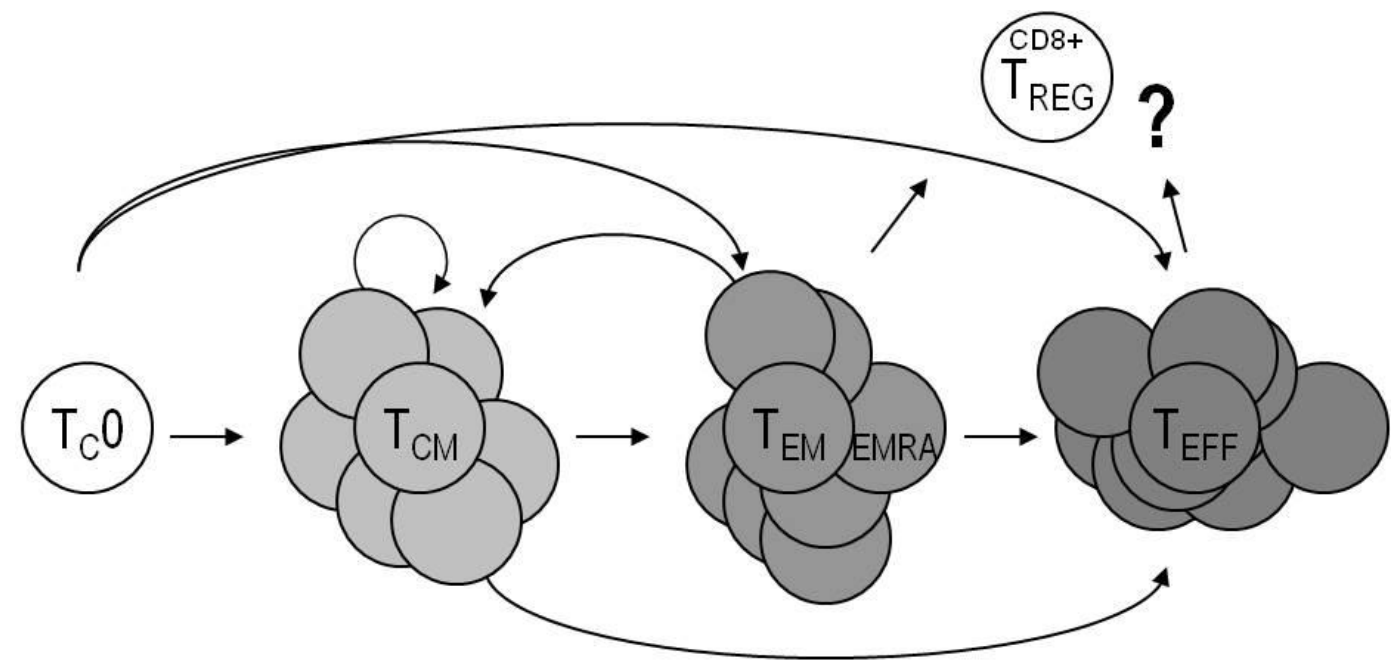

CD8+ $\mathrm{T}$ cell differentiation is poorly understood. The relationship between effector and memory cell generation and the regulatory CD8+ $\mathrm{T}$ cell lineage is less clear. This model is based on Figure 39, Figure 40, and Figure 41. 


\section{NOVEL FLOW-CYTOMETRY-BASED SUPPRESSION ASSAY}

Regulation of immunity is a fundamental concept in immunology. Many cellular phenotypes possess suppressor activity, including but not limited to the CD4+CD25+ regulatory T cells, CD4+ Tr1 cells, CD4+ Th3 cells, CD8+CD28-, HLA-E-restricted CD8+ T cells, CD8+TCR $\alpha \alpha+$ cells, among others. Mechanisms of suppression vary per model, subset, and condition, and many are left unresolved. Understanding the biology of human Treg is critical to the understanding of published and unpublished clinical data from intervention studies with suppressor cells. My method of measuring $\mathrm{T}$ cells suppressive ability, taking advantage of proliferation dyes and flow cytometry, will likely enable further studies in the fields allergy, transplant, and autoimmunity. 


\section{APPENDIX I}

\section{IMMUNE RESPONSES IN GENERAL}

The healthy human body mounts immune responses against cancercausing cells, microbes, parasites, and allergens through innate and adaptive (learned) mechanisms [314]. The innate immune system acts as first line defense against pathogen-associated patterns, broadly supporting the adaptive immune system. Effective adaptive immune responses involve the differentiation of antigen-specific $\mathrm{T}$ lymphocytes ( $\mathrm{T}$ cells) and B lymphocytes (B cells). T cells express the antigen-receptor, known as the $\mathrm{T}$ cell receptor (TCR) and CD3 signaling complex, and are classified into two major groups, known as helper $\mathrm{T}$ (Th) cells and cytotoxic $\mathrm{T}(\mathrm{Tc})$ cells. The antigen-receptors of lymphocytes are responsible for the memory, diversity, and specificity of adaptive immunity. B cells express on the cell surface the membrane bound antigen-receptor, known as the $\mathrm{B}$ cell receptor (BCR), and secret a form of the antigen-receptor, known as immunoglobulin (Ig) or antibodies.

\section{INNATE IMMUNITY}

The common theme of the innate immune system is pathogen-associated molecular patterns (PAMP) engaging pattern recognition receptors (PRR), such as the Toll-like receptors (TLR). In other words, the innate immune system is preprogrammed per each cell type of each species toward a limited set of common antigens. For example, human dendritic cells express a repertoire of approximately $10^{1}$ TLRs which are encoded in the host DNA, in order to recognize and attack highly-conserved motifs of bacteria, viruses, and fungi. 
Albeit limited as a collection of variability, the innate immune system makes up for its limited range with consistency, dependability, and few-adverse effects while targeting the most common microbial attributes. Many innate immune mechanisms are shared broadly throughout the eukaryotic phylogenetic tree. Despite a decreased number of genes among most invertebrates, the innate immune system provides high function per burden of genetic load. The adaptive immune system is not shared by organisms lower than jawless vertebrates, which lack the recombinase-activating gene (RAG) [315]. 
Figure 45: The adaptive immune system is not shared by organisms lower than jawless vertebrates, which lack the recombinase-activating gene

INNATE

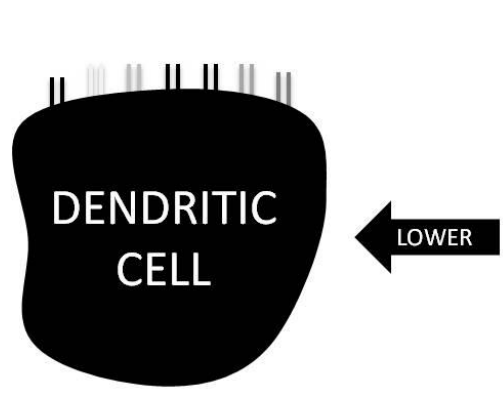

$10^{1}$ PATTERN-

RECEPTOR

SPECIFICITIES
VERTEBRATES

INNATE

$\stackrel{+}{\text { ADAPTIVE }}$

JAWLESS

VERTEBRATES

Plasma

$10^{12}$ CLONAL

ANTIGEN-

RECEPTOR

SPECIFICITIES 


\section{ADAPTIVE IMMUNITY}

The common theme of the adaptive immune system is lymphocytes bearing antigen-receptors generated by somatic recombination (SR). SR rearranges antigen-receptor gene segments in lymphocytes. The concept of clonal specificity of adaptive immunity describes how each lymphocyte expresses many of one type of antigen-receptor with affinity for one antigen. Adaptive immune responses are mounted by clonal expansion. The lymphocyte bearing the antigenreceptor of interest expands to form a population of lymphocytes bearing the same antigen-receptor. Therefore, each individual of a species has a unique representation of antigen-receptors among its pool of lymphocytes dictated by pathogen exposure and vaccinations. The total number of circulating lymphocytes and relative prevalences of antigen-receptors makes up the unique repertoire of the individual member of the species. The total number of possible antigen-receptor specificities per individual is estimated to be $10^{12}$ by taking into consideration the combined variability from the MHC and SR of the antigenreceptor [316]. Compared to the innate immune system, the adaptive immune system improves recognition and speed of repeated attack toward highly variable and unique motifs of pathogens, increasing with each encounter. The expanded range, customized specialization, and advanced memory of the adaptive immune system do not come without usage cost. DNA recombination carries the potential to introduce double strand breaks, chromosomal translocations, inversions, and other aberrations. Because of the chance of somatic recombination, many randomly generated antigen-receptor specificities are directed toward self. If left 
unchecked, self antigens may be misclassified as foreign, causing disease. In other words, the biological complexity required to increase the diversity of immunity carries with it the potential to produce autoreactive immune responses. Major histocompatiability complex (MHC) is critical to promoting self tolerance and acts as the antigen-receptor ligand for T cells. Distinction between self and foreign antigen is paramount to the adaptive immune system.

\section{T LYMPHOCYTES}

The two major groups of $\mathrm{T}$ cells are distinguished by the co-receptor to the antigen-receptor, known as TCR, expressed on the cell surface. The CD4 molecule is expressed by Th cells. The CD8 molecule is expressed by Tc cells. Th cells direct cell-mediated adaptive immune responses through the activation of macrophages and Tc cells in order to eliminate intracellular pathogens, such as viruses and intracellular bacteria, and neoplastic (cancer) cells. Th cells direct humor-mediated adaptive immune responses through the activation of antibodyproducing $\mathrm{B}$ cells in order to eliminate extracellular threats. When the mechanisms of innate and adaptive immune systems fail, the human body may be at risk of prolonged illness due to malignant cancer, opportunistic infections, allergies, or autoimmunity. While progress has been made in recent years to understand the innate and adaptive immune system, may aspects of immunology remain poorly understood. For this reason, investigators rely heavily on model systems to elucidate immune mechanisms that may translate into better treatments for people affected by illnesses that are immune-regulated and immunedeficiencies. During Th and Tc cell development and activation, loss in tolerance 
to self antigens may result in organ-specific autoimmune disease. A demyelinating disease of the CNS, multiple sclerosis, is thought to be immuneregulated. Prior studies have predominantly focused on models of autoreactive Th cells in autoimmunity, leaving Tc cells understudied and poorly understood. 
SUPPRESSIVE PROPERTY OF ACTIVATED CD4+CD25- T CELLS REVEALED BY A NOVEL FLOW CYTOMETRIC SUPPRESSION ASSAY

Prior studies by Pillai and colleagues in my lab showed that activated, dividing CD4+CD25- cells expressed FOXP3 under all the conditions tested. Suppressive property of activated $\mathrm{T}$ cells is still a matter of great controversy even after years of intensive study. While FOXP3 is currently the best marker to date of a regulatory $\mathrm{T}$ cell, it may co-incidently be a marker of activation. We reasoned that functional suppressive activity ought to supersede FOXP3 expression as the best measure of regulatory $\mathrm{T}$ cell function. To address the controversy, we developed a novel flow based suppression based on differential staining of cell populations, described in detail previously (Figure 46). Briefly, the CD4+CD25responders were stained with the green dye CFSE; the suppressors (induced Tregs or natural Tregs) with the dye CMTPX, and the APC's with the dye PKH. 
Figure 46: Novel flow cytometry based suppression assay uses differential staining of sorted populations to isolate and measure responder proliferation

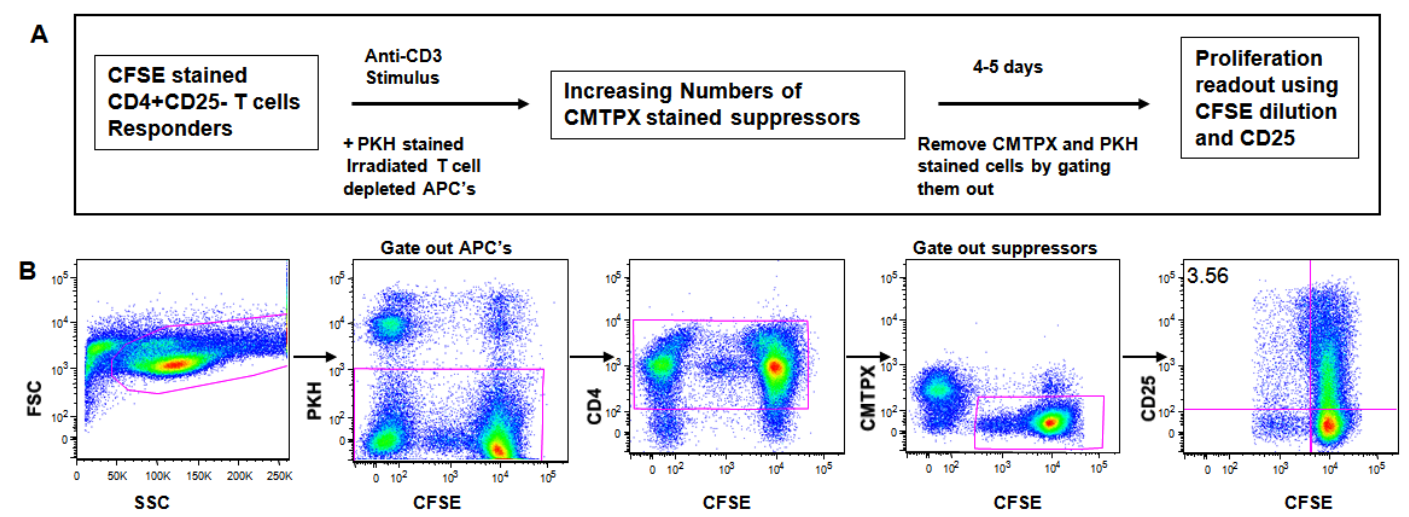

Assay design was representative of several variants designed and performed in collaboration with Dr. Vinodh Pillai (A) Schematic representation of the suppression assay set up. 0.5 million (M) CFSE stained CD4+CD25- T cells were used as responders in every tube. $0.5 \mathrm{M}$ CD4- $\mathrm{T}$ cells were stained with the red dye PKH, irradiated with 3500 rads were used as APC's. Varying ratios of CMTPX stained suppressors to responders (1:16 to 1:1) were added together along with APC's and antiCD3 and cultured for 5 days. (B) Gating strategy used to isolate and measure responder proliferation. After 5 days of culture cells were stained using CD4 PeCy5.5 and CD25 APC. Flow cytometric data was acquired on a BD LSRII. Proliferation of CD4+ responders was measured using CFSE dilution and CD25 expression after gating out the PKH stained APC's and CMTPX stained suppressors. \% Suppression is calculated considering the 'Responder only' proliferation as $100 \%$. 
We first tested this suppression assay in ex vivo natural Tregs and found that it robustly detects suppressive activity. We then showed that, using this suppression assay, the suppressive activity of activated CD4+CD25- cells was comparable or in most cases increased as evaluated against natural Tregs. Activation-induced suppressive ability was observed when different methods of activation were used: mixed lymphocyte reaction, anti-CD3 (Figure 47), staphylococcus enterotoxin B, PMA/ionomycin. 
SUPPRESSIVE ACTIVITY CORRELATES WITH ACTIVATION STATUS AND CD25 EXPRESSION

Another important question in the field is whether the suppressive activity is directly related to FOXP3 expression. Hence, we addressed that question by using the reliable and reproducible flow based suppression assay and correlating it with FOXP3 and CD25 expression. We found that suppressive activity was strongly correlated with activation, CD25 and FOX3 expression (Figure 47). The nonactivated CD4+CD25- $\mathrm{T}$ cells were not able to suppress the proliferation of the responders. It was also found at very late time points after activation when FOXP3 expression is really low, suppression also decreases (data not shown). 
Figure 47: Naturally-occurring CD4+CD25+ Tregs and activated FOXP3expressing ex vivo CD4+CD25- $T$ cells suppressed while non-activated ex vivo CD4+CD25-FOXP3- did not suppress autologous ex vivo CD4+CD25responders

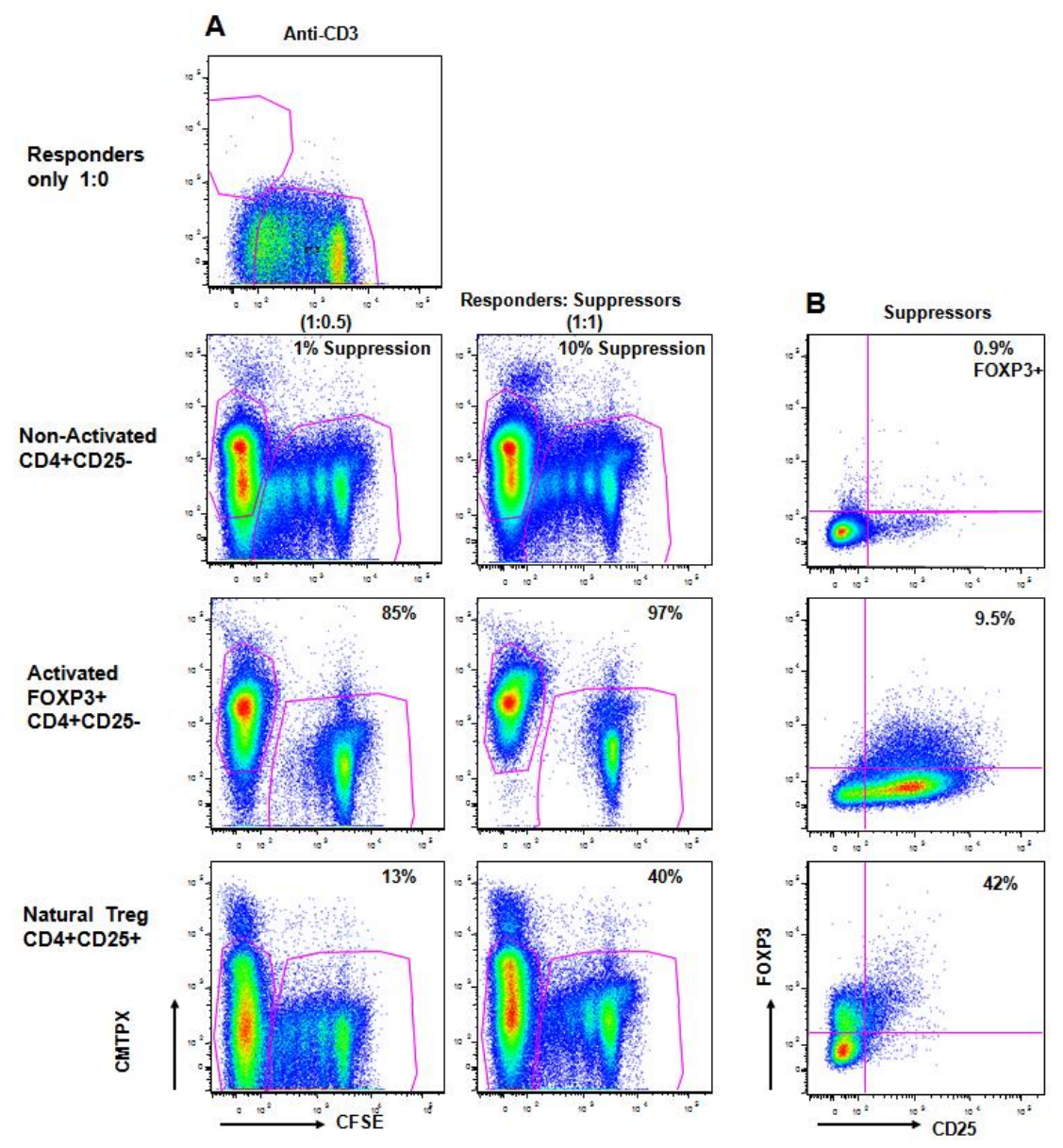

(A) CD4+CD25-FOXP3- $\mathrm{T}$ cells from healthy volunteers were activated with anti-CD3 for 5 days to induce FOXP3 expression and the suppressive ability of the activated cells was tested using the suppression assay. The dot plots show gated CD4+ $\mathrm{T}$ cells. While the number at top right corner shows the \% suppression calculated considering the dilution of CFSE in the top row responders only (1:0) as $100 \%$ proliferation. The second row shows CMTPX stained nonactivated CD4+CD25- along with CFSE-stained CD4+CD25- responders. The third row shows that the activated CD4+CD25- $T$ cells possess robust suppressive activity. As a positive control the suppressive ability of naturally-occurring $\mathrm{CD} 4+\mathrm{CD} 25+\mathrm{T}$ cells are shown in the bottom row. Our novel strategy of gating out APC's and suppressors using PKH and CMTPX showed the suppressive property of activated T cells. (B) FOXP3 and CD25+ expression are shown of cells used in suppression assay: non activated CD4+CD25- $\mathrm{T}$ cells, 5 day activated $\mathrm{CD} 4+\mathrm{CD} 25-\mathrm{T}$ cells, and naturally $\mathrm{CD} 4+\mathrm{CD} 25+\mathrm{T}$ cells. In the upper right hand corner is the \%FOXP3+. 
FOXP3 EXPRESSION IN ACTIVATED CD4+CD25- T CELLS IS NOT DUE TO TGF-B PRESENT IN HUMAN SERUM OR THAT PRODUCED BY ACTIVATED T CELLS OR APC

It has been suggested that FOXP3 expression in activated CD4+CD25- T cells is either due to TGF $\beta$ present in serum or TGF $\beta$ produced by activated T cells or APC's in cultures $[317,318]$. It has also been suggested that not all T cells can express FOXP3 on activation and the staining pattern seen is because the most commonly used FOXP3 antibody PCH101 can non-specifically stain activated T cells [319]. CD4+CD25-FOXP3- T cells were activated both in the presence and absence of serum containing media to compare their FOXP3 expression levels. X-vivo 15 (Cambrex Biosience, Walkersville, MD, USA) is a serum-free culture media which does not contain TGF $\beta$. The FOXP3 expression patterns under both conditions were essentially similar (Figure 48). In fact there was higher FOXP3 expression under serum free condition. 
Figure 48: FOXP3 expression by activated $T$ cells is not due to presence of TGF $\beta$ in serum or that secreted by activated $T$ cells
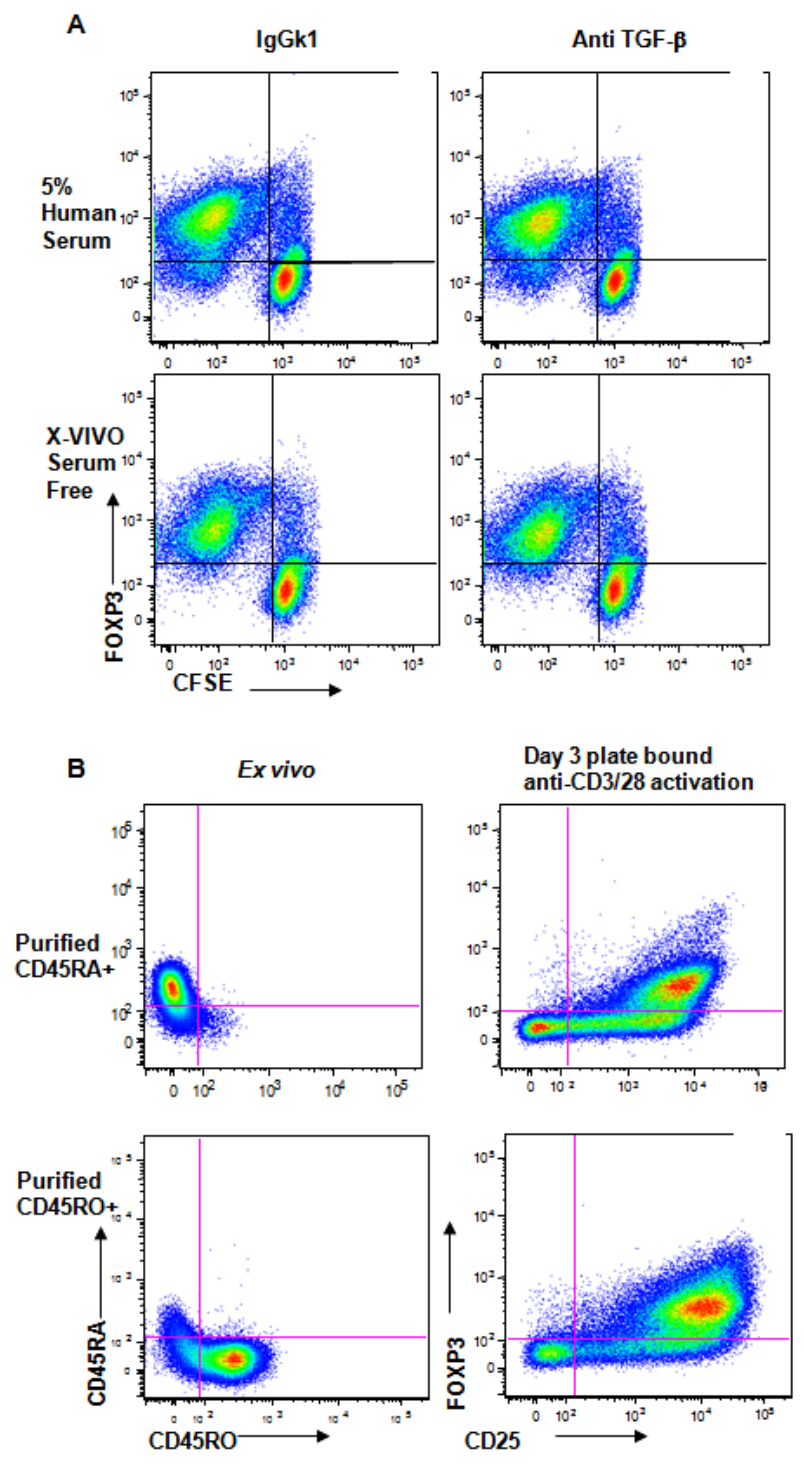

(A) CFSE-stained CD4+CD25- T cells stimulated with an allostimulus for 6 days in 5\% human serum-containing media and X-vivo serum-free media both in the presence and absence of anti-TGF $\beta$. (B) Bead sorted CD4+CD25-CD45RA+ naïve and CD4+CD25-CD45RO+ memory $\mathrm{T}$ cells were activated by plate bound anti-CD3 plus anti-CD28 for 3 days in $\mathrm{X}$-vivo serum-free media. 
SUPPRESSIVE PROPERTY OF ACTIVATED T CELLS IS CONTACT DEPENDENT AND IS NOT DEPENDENT UPON IL-2 CONSUMPTION, COMPETITION FOR APC OR NUTRIENTS IN MEDIA

It has been long been suggested that activated $\mathrm{T}$ cells do not suppress and whatever suppression is detected is probably artificial because of competition for IL-2, APCs or nutrients in media. These questions were addressed using the novel flow based suppression assay. Suppression assays were performed as described earlier but now in the presence of varying doses of recombinant human IL-2, excess APCs and media. We found that suppressive activity of activated T cells was still robust in all of the above conditions (Figure 49). Suppression of natural $\mathrm{T}_{\text {reg }} \mathrm{S}$ was not changed by addition of anti-TGF- $\beta$ or anti-IL-10. Similar to natural $\mathrm{T}_{\text {regs }}$, the suppression of activated T cells was not affected by anti-TGF- $\beta$ or antiIL-10. Addition of high dose IL-2 did not make a difference in the suppressive property of activated $\mathrm{T}$ cells. This suggests that the suppressive property is an active property of activated $\mathrm{T}$ cells and not a pseudo phenomenon due to passive factors. The suppressive activity of activated $\mathrm{T}$ cells was contact dependent, similar to that of nTregs (Figure 50). Cell free supernatants from MLR activated or anti-CD3 activated cultures were not able to suppress CD4+CD25- T cells further suggestive the suppressive activity is not a soluble factor (data not shown). Interestingly, high dose IL-2 was able to abrogate the suppressive activity of natural Tregs in parallel experiments suggesting there may be differences in the suppressive activity of nTregs and activation-induced Tregs. This might be a crucial difference between the natural $\mathrm{T}_{\text {regs }} \mathrm{s}$ and activated $\mathrm{T}$ cells because 
suppression of natural $\mathrm{T}_{\text {reg }} \mathrm{s}$ is known to be abrogated by addition of high dose IL2. 
Figure 49: Compared to nTreg, suppressive activity of activated CD4+CD25$T$ cells was increased and not affected by addition of anti-TGF $\beta$ or anti-IL-10 or high dose IL-2
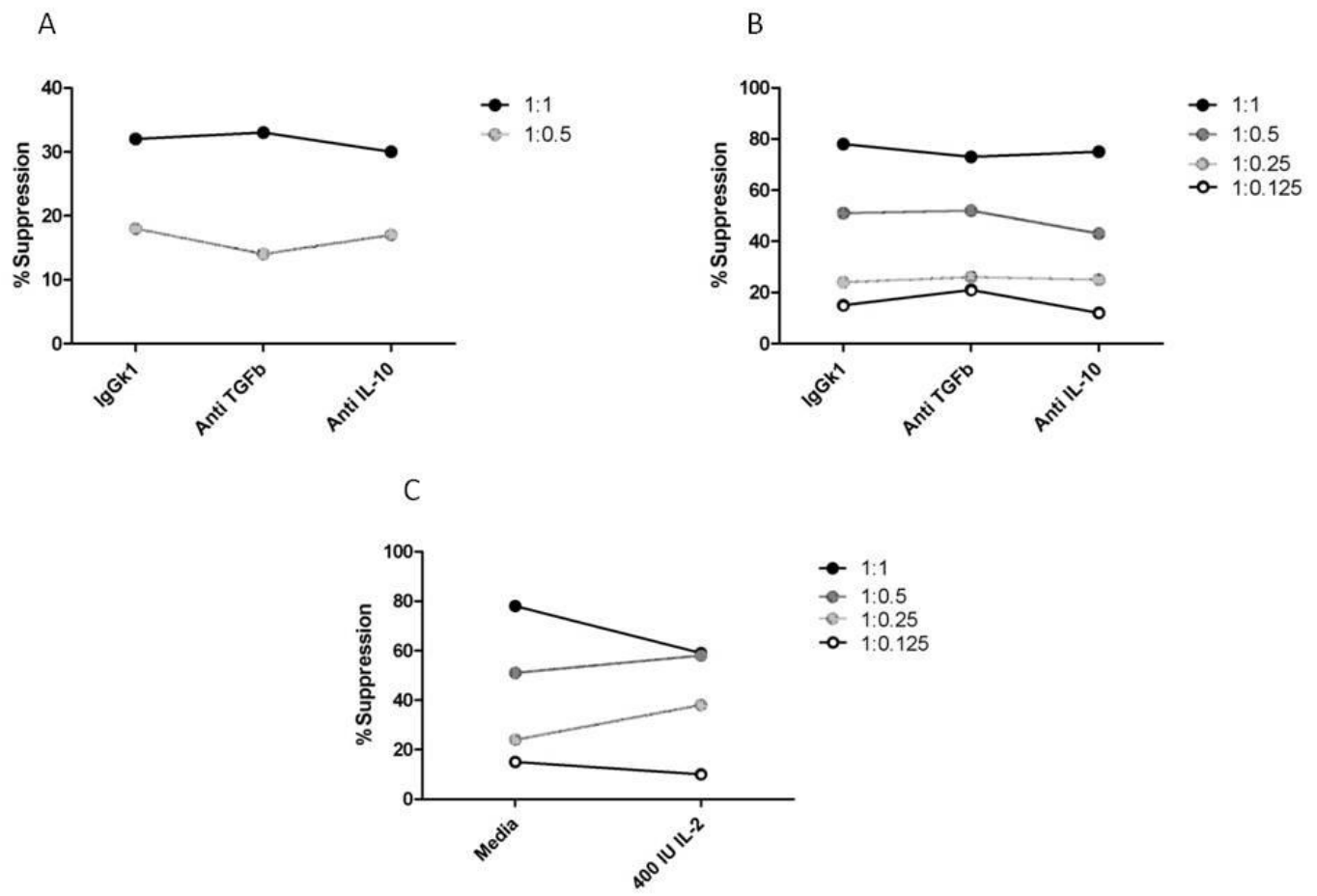

Experiments were representative of several designed and performed in collaboration with Dr. Vinodh Pillai. The effect of anti-TGF- $\beta$, anti-IL-10 and high dose IL-2 on the suppressive activity of natural $\mathrm{T}_{\mathrm{reg}} \mathrm{S}(\mathrm{A})$ and activated $\mathrm{T}$ cells (B and C) was examined using the flow based suppression assay. In all graphs the y axis shows the \% suppression calculated considering responders only proliferation as $100 \%$ while the $\mathrm{x}$ axis shows the different conditions. 
Figure 50: Suppressive activity of activated CD4+CD25- $T$ cells is contact dependent

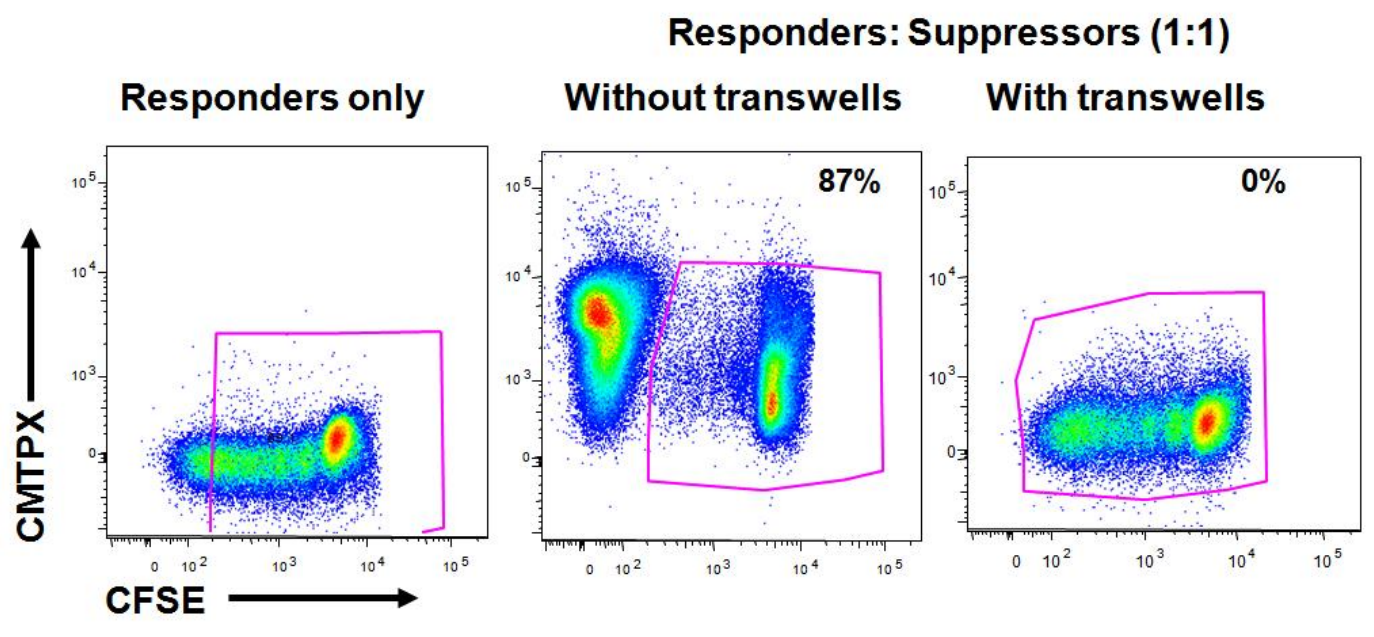

Suppression assays using 3 day anti-CD3 activated CD4+CD25- T cells were set up as described previously. In addition, 0.4 micron transwells were used to separate the responders from the suppressors to determine if contact is necessary for suppression. APC's and anti-CD3 were added both above and below the transwells to ensure activation of both responders and suppressors. CFSE vs CMTPX dot plots from such suppression assays are shown here. Suppressors and responders were mixed in a 1:1 ratio in the absence and presence of transwells. The numbers in the dot plots indicate the \% suppression in each of those cocultures. Presence of the transwell abrogates the suppressive activity of activated $\mathrm{CD} 4+\mathrm{CD} 25-\mathrm{T}$ cells suggesting that contact is necessary for suppression. Combined with results from figure 3 , this suggests that similar to the suppressive activity of natural $\mathrm{T}_{\text {regs }} \mathrm{s}$, suppressive activity of activated CD4+CD25- $\mathrm{T}$ cells is also mediated by a membrane bound or membrane transferred contact dependent factor. 
ANTIGEN NAÏVE AND MEMORY T CELLS EXPRESS FOXP3 UPON ACTIVATION AND ACQUIRE SUPPRESSIVE PROPERTIES

Most $\mathrm{T}$ cells in adults are antigen experienced cells and our question may be better addressed in antigen naive T cells. Other studies have observed FOXP3 induction in naïve $\mathrm{T}$ cells under APC free conditions using plate bound antiCD3/28 [319]. Memory cells in the CD4+CD25- population may also show inducible FOXP3 expression. In our hands, naïve CD45RA+ cells and memory CD45RO+ cells expressed FOXP3 upon activation using both plate bound antiCD3 and serum free x-vivo media (Figure 51). Hence, we investigated the FOXP3 expression in CD4+CD25- T cells in cord blood cells. FOXP3 was still expressed even when a nearly antigen naïve populations under plate bound conditions were used (Figure 11). Anti TGF- $\beta$ was also added to neutralize any TGF- $\beta$, making no difference to the FOXP3 expression pattern. This suggests that FOXP3 expression by activated naïve $\mathrm{T}$ cells cannot be solely attributed to the presence of TGF- $\beta$ in serum or that produced by other activated T cells. Other factors are more likely to influence induced FOXP3 expression. 
Figure 51: Cord blood naïve T cells express FOX3 upon activation by plate bound anti-CD3/anti CD28 in serum free $\mathrm{X}$-vivo media
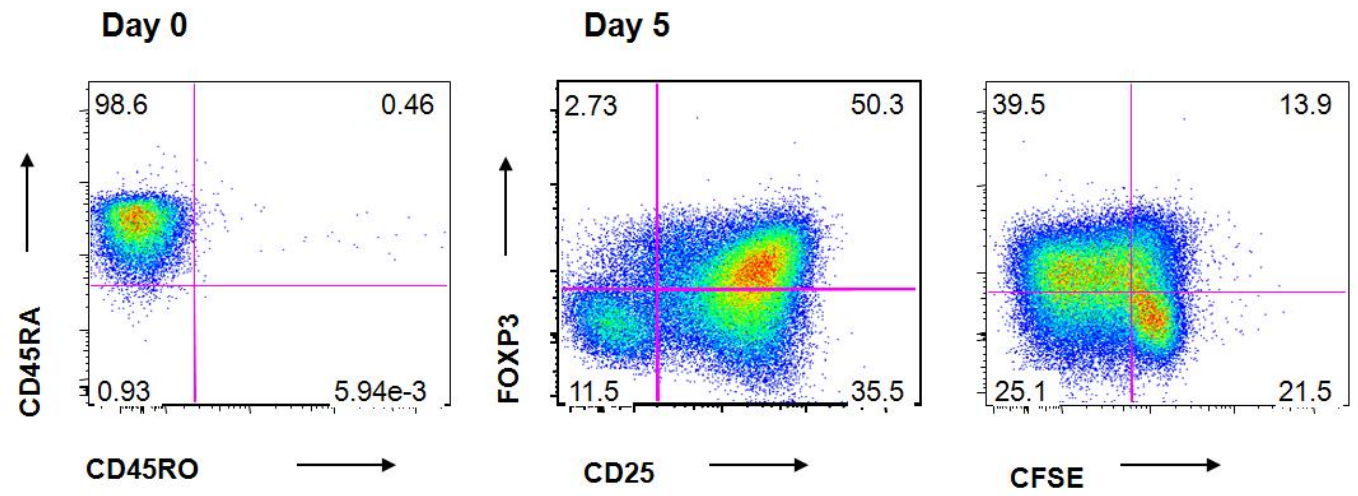

Bead sorted CFSE stained CD4+CD25-CD45RA+ naïve (99\% pure) from human cord blood were activated by plate bound anti-CD3+anti-CD28 for 5 days in serum free $\mathrm{X}$-vivo media. Most activated $\mathrm{T}$ cells can be seen to express FOXP3 on activation suggesting that this phenomenon is not unique to adult humans who have a predominance of memory $\mathrm{T}$ cells in their blood. 
Activation of antigen-naïve CD4+CD25- T cells from cord blood induced FOXP3 expression and suppressive properties in those cells (data not shown). CD4+CD25-CD45RA+ T cells and CD4+CD25-CD45RO+ T cells each showed suppressive activity upon activation (data not shown). Activated cord blood $\mathrm{FOXP}^{+}$T-cells exhibit robust and highly reproducible suppressive activity even at very low suppressor to responder ratios. Similar to natural regulatory T-cells, their suppression is contact dependent and anti-TGF $\beta$ and anti-IL-10 independent. Suppression is not due to IL-2 consumption since addition of high dose exogenous IL-2 did not make any difference to suppression. In conclusion, we showed the suppressive nature of activated, FOXP3-expressing T-cells and introduced an assay system that will be highly useful in dissecting the biology of induced regulatory T-cells. 


\section{EFFECT OF KNOCKING OUT FOXP3 EXPRESSION IN HUMAN T CELLS}

We then tried to knock out FOXP3 by RNA interference (RNAi). Vinodh was able to achieve maximal knockdown FOXP3 expression in activated $\mathrm{T}$ cells by pulsing CD4+CD25- cells with FOXP3 specific short interfering RNA (siRNA) before activation (data not shown). We were not able to perform suppression assays with siRNA treated cells since the duration of siRNA action was only 36$48 \mathrm{hrs}$, and our flow based suppression assays is 5-7 days of culture. Vinodh and I also attempted to demonstrate shRNA-mediated knockdown of both activated and natural FOXP3 as described earlier. These experiments were largely inconclusive as polybrene and/or infection of the primary lymphocytes with control scrambled shRNA virus altered their activation status and/or suppressive ability (Figure 52, Figure 53, Figure 54). 
Figure 52: Treg-depleted CD4+CD25- $T$ cells were resistant to puromycin treatment after shRNA-lentivirus infection

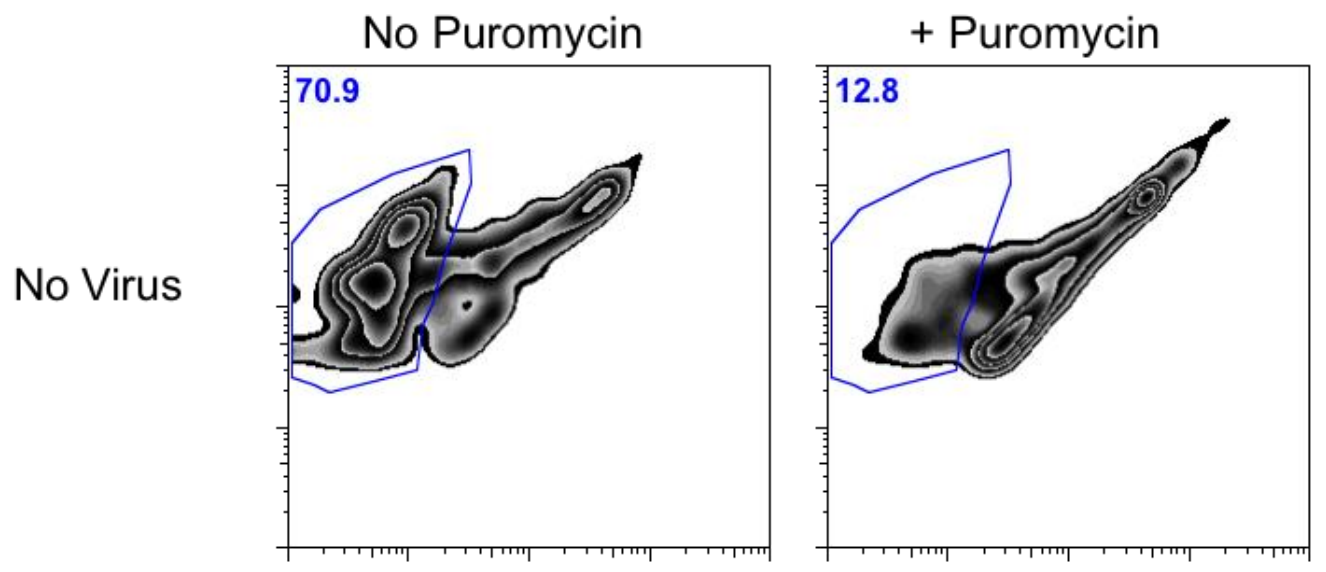

FOXP3 shRNA virus

Scramble shRNA virus
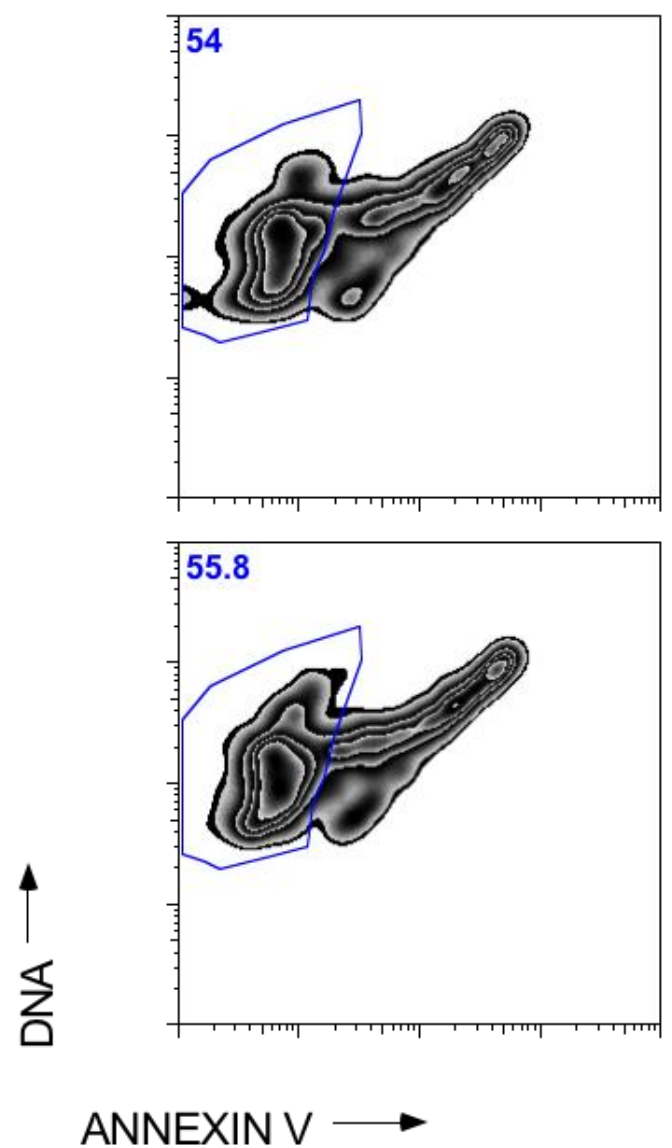
Figure 53: FOXP3 shRNA lentivirus achieves partial protein knockdown of FOXP3
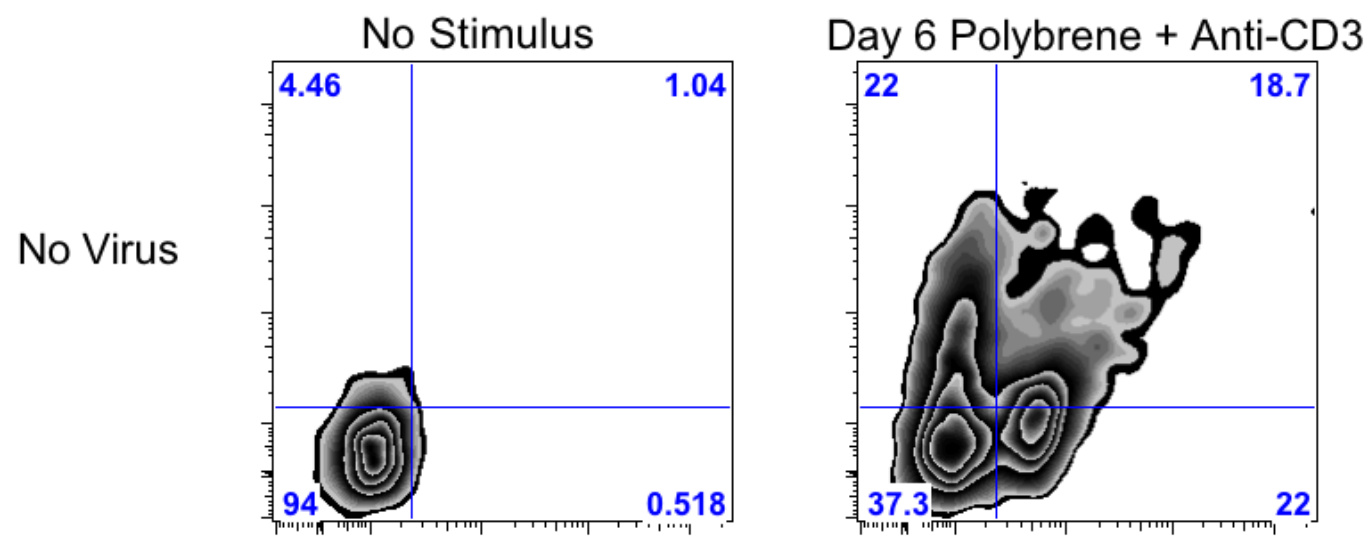

FOXP3 shRNA Virus

Scramble shRNA Virus
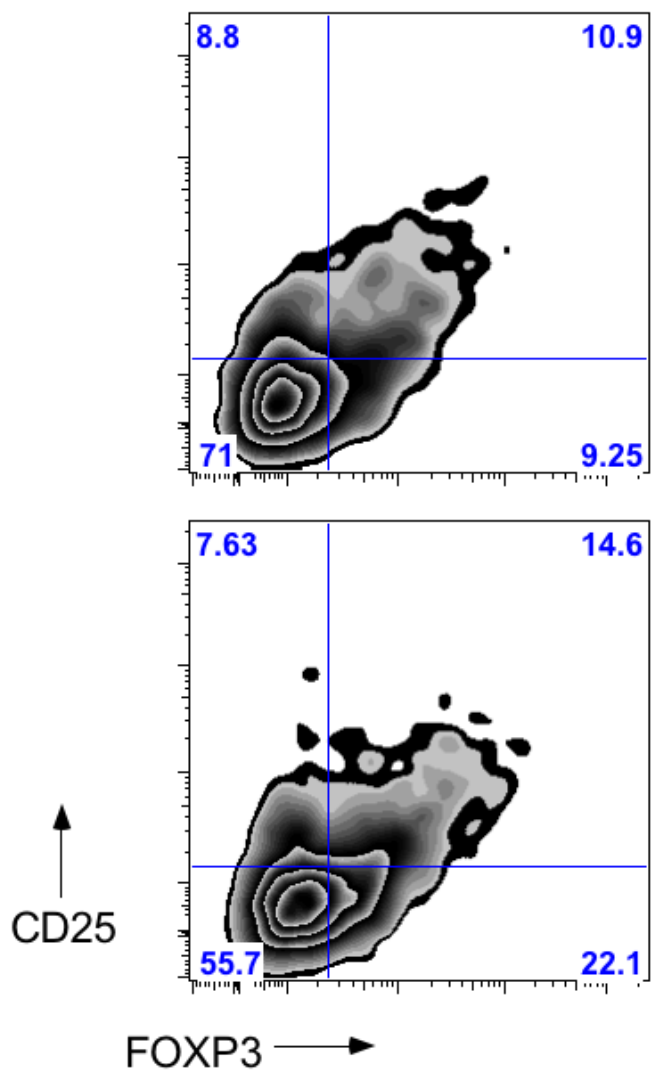
Figure 54: Polybrene-treated iTreg suppressive ability is not detectable by flow-based suppression assay

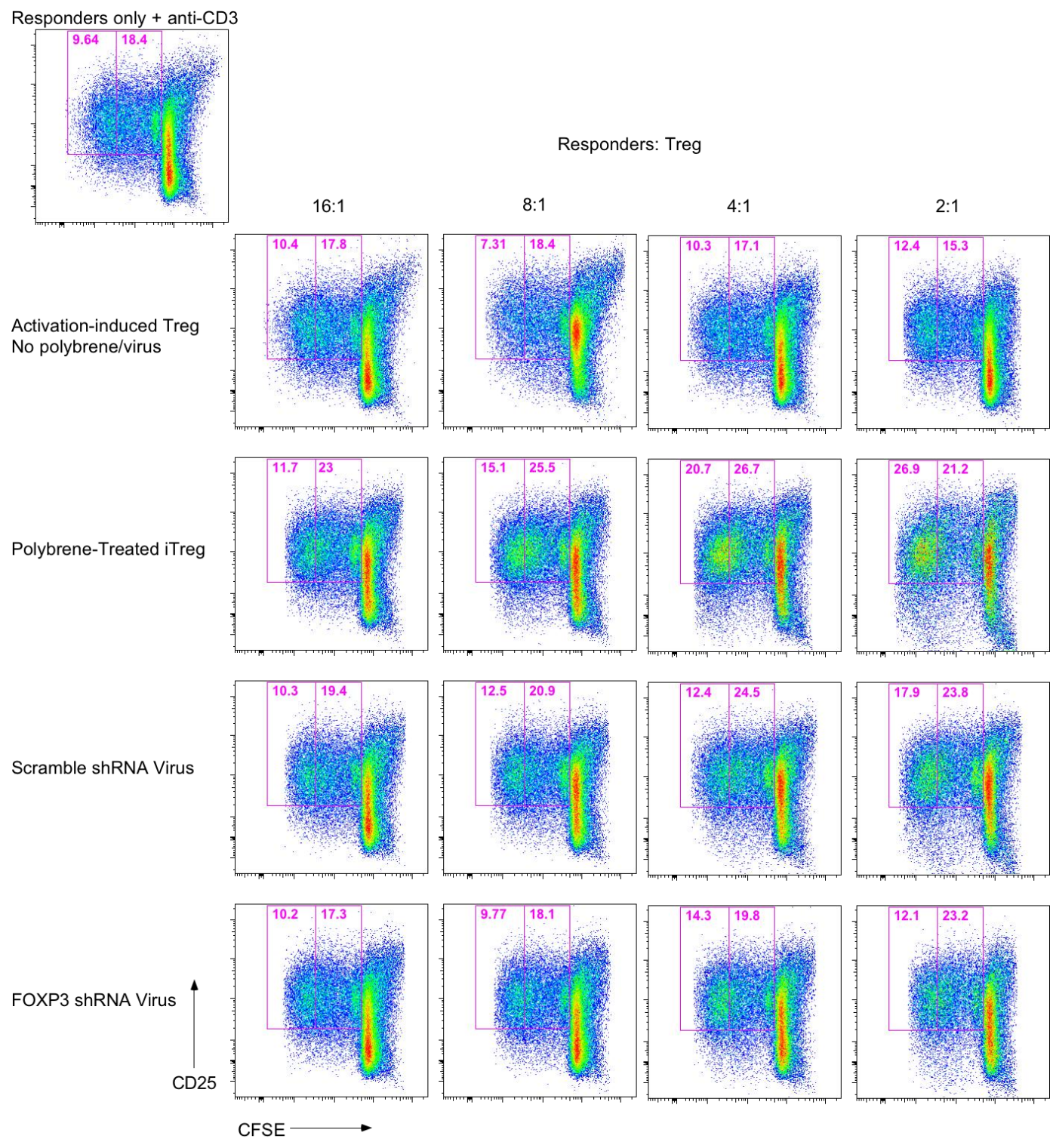

FOXP3 shRNA virus and scrambled shRNA virus were used to test the role of FOXP3 in activation-induced suppression. Polybrene was used as a cotransfection agent. The untreated activation-induced Tregs control and the polybrene-treated iTreg control failed to suppress autologous responder cells as expected. Technical difficulties prevented the furtherance of this knockdown strategy. 


\section{DISCUSSION}

We devised a novel flow cytometry-based suppression assay, whereby the putative suppressor cells were excluded from the analysis of proliferation, avoiding the pitfalls of the thymidine-based assay through taking advantage of proliferation dyes and long-term cellular tracking dyes. Using this assay, CD4+ regulatory $\mathrm{T}$ cells were characterized to great length. We showed that the flow cytometry based suppression assay consistently detects suppressive activity in activated $\mathrm{T}$ cells. As the thymidine based suppression assay measures the total proliferative activity of all the cells in culture, the suppressive activity of activated cells could may be masked by their proliferation especially when the suppressors are not anergic. This phenomenon may be responsible for the failure of early Treg experiments to detect the suppressive activity of activated T cells. Many papers also do not use purified population of CD25+ $\mathrm{T}$ cells from activated $\mathrm{T}$ cell cultures. The suppressive property of activated $\mathrm{T}$ cells can be missed by the insensitivity of the thymidine based suppression assay and not by the flow based suppression assay.

The novel suppression assay showed that activated $\mathrm{T}$ cells clearly possess suppressive properties at even very low ratios. Suppression was not due to overcrowding of cells as there is no suppression by non-activated CD4+CD25- or freshly isolated CD4+CD25- T cells. Activation-induced suppression was not due to IL-2 consumption because even high doses of IL-2 did not abrogate suppression in contrast to nTreg. Suppression was clearly contact-dependent and was not affected by even high doses of soluble anti-TGF $\beta$ or anti-IL-10. FOXP3 
expression by all activated human T cells was seen even when adult or cord blood CD4+CD25-CD45RA+ naïve T cells in serum free X-vivo media and plate bound anti-CD3/anti-CD28 was used.

One of the best-understood subsets of immune regulatory cells is the CD4+CD25+ Treg. FOXP3+ cells in mouse are thought to define Treg. FOXP3 represses the transcriptional activity of NFAT and NFkb among other mechanisms. The role of FOXP3 in humans is thought to be more complex. Ex vivo CD4+CD25-FOXP- T cells, upon activation, express FOXP3 and possess suppressive ability. Whether this holds to be true in mouse cells is currently controversial and is being investigated by collaborators in my lab. Previous notions about naturally-occurring Treg from the thymus may be re-examined by these new findings. Human Tregs, as a lineage, could be expanded from an existing pool or induced de novo in the periphery. Most evidence supports the former possibility in mice while human Tregs are probably derived by a combination or mostly the latter. Generation of human Tregs is important to the extent that many studies have proposed in vitro expansion and therapeutic adoptive transfer in diseases of hyper-immune activation (autoimmunity, allergy, asthma). If human FOXP3+ cells represent only a transient population, then harmful or sub-therapeutic results may be explained. FOXP3 status must be interpreted in light of immune activation as a product of the FOXP3 level.

These and other findings address a matter of great controversy as to whether activated $\mathrm{T}$ cells possess suppressive properties. While we and others have shown previously that they do indeed possess suppressive properties, many 
others were not able to detect the suppressive property. We hypothesized that the differences in the results obtained by different groups is due to the peculiarities of the widely used thymidine based suppression assay. The thymidine based suppression assay measures the total proliferation of all cells in culture. Hence if the suppressive population in question is not anergic then it would not be able to detect the suppressive activity.

One reason for disparate results in measuring the suppressive property of activated cells could be the wide use of thymidine based suppression assays which fail when the suppressor population is not anergic. We directly compared the performance of the two assays in detecting suppressive property of activated $\mathrm{T}$ cells and natural Tregs. We found that while the performance of the two assays was comparable when the putative suppressors were anergic; performance was dramatically different when the putative suppressors were not anergic. This was especially true in the case of activated cells since by definition they are not anergic when they are activated. Hence, it would be very hard to measure their suppressive property during that phase. Our assay would also work well in the case of natural Tregs since it is difficult to obtain a pure population of anergic Tregs. We showed that our assay would be vastly superior in measuring the suppressive property compared to thymidine based suppression assays even when the population is a mixed population of natural Tregs and other contaminating cells.

DISTINCTIONS BETWEEN ITREGS AND NTREGS 
Addition of high dose IL-2 did not make a difference in the suppressive property of activated $\mathrm{T}$ cells. This might be a crucial difference between the natural $\mathrm{T}_{\mathrm{reg}} \mathrm{S}$ and activated $\mathrm{T}$ cells because suppression of natural $\mathrm{T}_{\mathrm{reg}} \mathrm{S}$ is known to be abrogated by addition of high dose IL-2. Natural Tregs lose expression of CD127 and therefore dependent upon IL7 for survival. Dependence upon IL7 may distinguish natural from activation induced regulatory $\mathrm{T}$ cells. 


\section{PRIONS IN T CELL ACTIVATION}

Prion protein $(\mathrm{PrP})$ or prions are responsible for the transmission of spongiform encephalopathies [320]. Unlike most other infectious agents such as viruses, PrP lacks any nucleic acid and is simply a modified form of a normal protein found in mammals. The misfolded $\operatorname{PrP}$ configuration $\left(\mathrm{PrP}^{\mathrm{sc}}\right)$ is associated with animal prion disease, scrapie, in mammals and Creutzfeld-Jacob disease (CJD) in homosapiens. Spongiform encephalopathies are rare in both humans and their domesticated large mammals. All forms of prion disease affect approximately one per million people in the US. The bovine form of spongiform encephalopathy is popularly known as mad-cow or foot-and-mouth disease. Histopathologically, spongiform encephalopathies exhibit microscopic vacuoles within neurons and their processes. The abundant vacuoles give a sponge-like appearance. $\operatorname{PrP}^{\mathrm{sc}}$ is arranged as polymers of $\beta$-pleated sheets, forming deposits. Amyloid deposits may contribute to neuronal loss, atrophy, reactive gliosis, and inflammatory infiltration.

\section{TRANSMISSION}

Unlike any other infectious agent known, PrP disease may occur sporadically, be transmitted from one host to another (by neurosurgical instruments or tissue transplants), or be inherited in an autosomal dominant manner. Lacking nucleic acids, $\mathrm{PrP}^{\mathrm{sc}}$ is resistant to $\mathrm{UV}$ radiation, high temperature and pressure, and many chemical disinfectants that are otherwise effective on most infectious agents. Sporadic CJD most often affects individuals in their sixties. Most cases of 
transmitted prion disease occurred in the UK through the ingestion of cattle with mad-cow disease. Fatal familial insomnia is an inherited form.

\section{CLINICAL PRESENTATION}

The symptoms of prion disease classically include, but are not limited to the following: rapidly progressive dementia (less than a year), ataxia, jerking movement, blindness, behavioral and psychiatric disturbances, and neuropathic pain. Prion disease is always fatal, usually within the first year after diagnosis.

The physiological function of PrP is unknown. Based upon its abundance, normal cellular protein likely plays an important physiological role. Contrarily, animals lacking $\operatorname{PrP}$ are viable. Most problematic toward their study, $\operatorname{PrP}^{\mathrm{c}-/-}$ mice are relatively normal with merely subtle abnormalities. The PrP cellular form $\left(\operatorname{PrP}^{\mathrm{c}}\right)$ is found in healthy individuals and is expressed ubiquitously, but highly in the CNS and hematopoetic cells, including myeloid dendritic cells [321]. Upon activation, $\operatorname{PrP}^{\mathrm{c}}$ is up-regulated on $\mathrm{T}$ cells [322]. Once $\operatorname{PrP}^{\mathrm{c}}$ misfolds into $\operatorname{PrP}^{\mathrm{sc}}$, the presence of $\operatorname{PrP}^{\mathrm{sc}}$ acts as a catalyst, converting more $\operatorname{PrP}^{\mathrm{c}}$ into $\operatorname{PrP}^{\mathrm{sc}}$, primarily in nervous tissue. $\operatorname{PrP}^{\mathrm{c}}$ is a highly conserved 36 kilodalton transmembrane protein, consisting of $\alpha$-helices, and is anchored by its C-terminus to a glycosylphosphatidylinositol (GPI) in lipid rafts. On its N-terminus are oligosaccharide complex oligosaccharide chains [323]. While PrP is not truly infectious as a virus, the aberrantly folded protein particles can be spread from one afflicted individual to another. Many studies have sought to uncover the physiological functions of $\operatorname{PrP}^{\mathrm{c}}$ [324]. A selected few are reviewed here. 
Many proteins are candidates for interaction with $\operatorname{PrP}^{\mathrm{c}}$. Yeast two-hydrid screens, co-immunoprecipitation, and cross-linking studies have identified lists of putative $\operatorname{PrP}^{\mathrm{c}}$ interactors. All of the candidate's physiological relevance to $\operatorname{PrP}^{\mathrm{c}}$ remain uncertain. The smallest fraction of transmembrane $\operatorname{PrP}^{\mathrm{c}}$ is cytoplasmic. Therefore many have been unlikely candidates, not being transmembrane or secreted proteins. Like most other GPI-anchored proteins residing in the lipid raft, $\operatorname{PrP}^{c}$ may play an important role either directly in signal transduction or indirectly as scaffolding for such processes [325].

\section{PHYSIOLOGICAL ROLE OF CELLULAR PRION PROTEIN}

Compelling while insufficient evidence suggests that $\operatorname{PrP}^{\mathrm{c}}$ may possess a role in neurons. As prion disease pathology occurs primarily in the brain, it stands to reason that the major focus of study has been at the site. Immortalized neurons from mice lacking $\operatorname{PrP}^{c}$ are more susceptible to serum deprivation-induced apoptosis. In serum deprivation conditions, $\operatorname{Pr}^{\mathrm{c}-/-}$ cells maintain mirochondial cytochrome c and membrane potential levels as compared to wild type cells [326]. PrP neuroprotection may involve inhibition of the mitochondrial proapoptotic pathways. The moiety of serum responsible for signaling to $\mathrm{PrP}^{\mathrm{c}}$ or the intracellular target of $\operatorname{PrP}^{\mathrm{c}}$ is unknown. The intracellular binding partner may have been already identified as one of the putative interactors. Antibodies are thought to bind $\operatorname{PrP}^{\mathrm{c}}$, which leads to dimerization of $\operatorname{PrP}^{\mathrm{c}}$ at the plasma membrane and phosphorylation of extracellular regulated kinase (ERK1/2), promoting neuronal survival. Decreased endogenous $\operatorname{PrP}^{\mathrm{c}}$ expression in neurons has been associated with increased bax-mediated cell death [327]. Contrarily, over- 
expression of $\operatorname{PrP}^{c}$ in some cell line increases susceptibility to apoptosis to staurosporine through the action of caspase 3 [328, 329]. Over-expression in MCF7 breast cancer cell lines increases resistance to TNF $\alpha$ [330]. It is unclear if over-expression of PrP may lead to a conversion to the lethal $\operatorname{PrP}^{\mathrm{sc}}$ conformation. $\mathrm{PrP}^{\mathrm{c}-/-}$ neuronal cell lines resist the oxidative damage caused by copper [331]. $\operatorname{PrP}^{\mathrm{c}}$ may act to detoxify the brain from the effects of copper. Whether $\operatorname{PrP}^{\mathrm{c}}$ possesses superoxide dismutase activity or modulates the activity is still being debated [332, $333 \mathrm{C}, 334]$. $\operatorname{PrP}^{\mathrm{c}}$ may play a critical role in the balance between neuroprotective and neurotoxic activity in the brain. A large, but rather inconclusive, body of evidence points to a role of $\operatorname{PrP}^{\mathrm{c}}$ in regulating neuron survival, differentiation, growth, synapse formation, cell adhesion, and apoptosis. Other cell types that highly express $\operatorname{PrP}^{\mathrm{c}}$ are still understudied in this regard. In consideration of the expression of $\operatorname{PrP}^{\mathrm{c}}$ in the blood, bone marrow, skin [335], stomach [336], breast, and kidneys [337], alternative hypotheses of its physiologic function have been proposed. Hematopoesis, inflammation, bacterial infection, and $\mathrm{T}$ cell activation involvement have been suggested as the function of $\operatorname{PrP}^{\mathrm{c}}$ [338]. $\operatorname{PrP}^{c}$ may act in the innate or adaptive immune system as a microbial pattern recognition receptor, similar but independent to TLR/NOD. The Nterminus cationic and heparin-binding domain of recombinant $\operatorname{PrP}^{\mathrm{c}}$ has an antimicrobial effect toward S. aureus ATCC 29213, E. coli ATCC 25922, Pseudomonas aeruginosa ATCC 27853 and C. parapsilosis ATCC 90018 isolates [339]. The peptide disrupted the membranes of the microorganisms. The $\mathrm{N}$-terminus of $\operatorname{PrP}^{\mathrm{c}}$ has been shown to be cleaved by oxidative stress or reactive 
oxygen species [340]. Similar to kininogen and other heparin-binding peptides, cleavage or release of the N-terminus of $\operatorname{PrP}^{\mathrm{c}}$ could play an important antibacterial role during the innate immune response during an infection [341, 342]. This hypothesis may more plausibly account for the ubiquitous expression of $\operatorname{PrP}^{\mathrm{c}}$ in the skin, gut, lymphoid tissue, brain, blood, and kidney.

\section{ROLE OF PRIONS IN T CELLS}

Following stimulation with the mitogen concanavalin A (ConA), activated $\mathrm{T}$ cells up-regulated $\operatorname{PrP}^{c}$ [343]. ConA induced-lymphocyte proliferation was diminished in mouse $\mathrm{T}$ cells lacking $\operatorname{PrP}^{\mathrm{c}}$. Mice lacking $\operatorname{PrP}^{\mathrm{c}}$ display more clinically severe EAE, suggesting that $\operatorname{PrP}^{\mathrm{c}}$ may possess an important role in $\mathrm{T}$ cell immune modulation [344]. Leukocyte infiltration into the spinal cord, cerebellum, and forebrain was increased in mice after were immunized with MOG. MOG-primed $\mathrm{T}$ cells and macrophages/glia from $\mathrm{PrP}^{\mathrm{c}}$-deficient mice with EAE expressed more IFN $\gamma$ and iNOS, respectively, in spinal cords compared to controls. TNF $\alpha$ and IL-1 $\beta$ were not significantly affected in spinal cords. In the $\operatorname{PrP}^{\mathrm{c}-/-}$ forebrain and cerebellum, transcripts for IFN- $\gamma$, TNF- $\alpha$, IL- $1 \beta$, iNOS, and RANTES were increased in during EAE compared to controls.

$\mathrm{T}$ cell activation and TCR signaling may regulated by $\operatorname{PrP}^{\mathrm{c}}$ [345]. Using RNA interference (RNAi) in mice, $\operatorname{PrP}^{c}$ expression was decreased by 70 percent in peripheral leukocytes, leaving CNS expression unaffected. $\operatorname{PrP}^{c}$ RNAi mice suffered clinically severe EAE compared to control mice. Sub-optimal PLP p139-151 $_{1}$ immunization during EAE induction was required in order to reduce mortality to that of control mice. Antigen-specific T cell proliferation, activation, and survival 
were increased through $\operatorname{PrP}^{c}$ RNAi. Transgenic TCR mice with T cells specific to $\mathrm{MBP}_{1-11}$ acquired spontaneous EAE when treated with $\operatorname{PrP}^{\mathrm{c}} \mathrm{RNAi}$, but not when treated with scrambled RNAi. $\operatorname{PrP}^{\mathrm{c}}$ RNAi in combination with anti-CD3/antiCD28 treatment enhanced TCR signaling by up-regulating zeta-chain-associated protein-70 (ZAP70) phosphorylation and nuclear factor of activated $\mathrm{T}$ cells/activator (NFAT) protein 1 transcriptional activity. $\operatorname{PrP}^{\mathrm{c}}$ RNAi mice had increased differentiation of myelin-specific T cells towards Th1 and Th17 cells, while APC were not affected by $\operatorname{PrP}^{\mathrm{c}}$ RNAi. $\mathrm{MBP}_{1-11}$ TCR transgenic mice developed spontaneous EAE when treated with $\operatorname{PrP}^{\mathrm{c}}$ RNAi. Mice that overexpress $\operatorname{PrP}^{\mathrm{c}}$ had reduced clinical severity of EAE. Other studies have suggested that $\operatorname{PrP}^{\mathrm{c}}$ participates in $\mathrm{T}$ lymphocytes activation [322, 346, 347]. Likewise, using human cell, I showed that anti-CD3 stimulated CD4+ T cells upregulated $\operatorname{PrP}^{\mathrm{c}}$ and that $\operatorname{PrP}^{\mathrm{c}}$ expression was correlated with $\mathrm{CD} 25$ and FOXP3 expression. This suggests that $\operatorname{PrP}^{\mathrm{c}}$ may possess a role as a negative regulatory of proximal TCR signaling in human CD4+ Tregs while limiting Treg survival. The precise roles of $\operatorname{PrP}^{c}$ and especially $\operatorname{PrP}^{\mathrm{sc}}$ in $\mathrm{T}$ cell activation remain poorly understood and under-appreciated.

How $\mathrm{T}$ cell activation may play a role in the abnormal $\operatorname{PrP}^{\mathrm{sc}}$ configuration is poorly understood. Severe combined immunodeficiency (SCID) mice challenged with $\mathrm{PrP}^{\mathrm{sc}}$ are resistant to intraperitoneal and subcutaneous inoculation while sensitive at a low level disease after intracebrebral injection [348]. Immunocompetent or bone-marrow reconstituted SCID mice were highly susceptible to prion disease, suggesting that normal lymphoid structure is required 
for transmission and infectivity of $\operatorname{PrP}^{\mathrm{sc}}$. Mice that lack $\operatorname{PrP}^{\mathrm{c}}$ expression in all tissues except $\mathrm{T}$ cells and over-express $\operatorname{PrP}^{\mathrm{c}}$ on $\mathrm{T}$ cells are resistant to scrapie [349]. Cell-mediated immune responses appear unaffected in scrapie mice [350]. Scrapie mice lack a detectable $\operatorname{PrP}^{\mathrm{sc}}$-specific immune response. $\operatorname{PrP}^{\mathrm{sc}}$ may accumulate in splenic follicular dendritic cells (FDC) where it is carried to lymph nodes and transmitted to the CNS $[351,352]$. How carrier FDCs interact with T cells and nervous cells in order to spread $\operatorname{PrP}^{\mathrm{sc}}$ remains unclear. 
PRPC IS EXPRESSED UBIQUITOUSLY BY HEMATOPOETIC CELLS AND HIGHEST IN A SUBSET OF ACTIVATED/REGULATORY T LYMPHOCYTES

Figure 55: PrPc is expressed ubiquitously by hematopoetic cells and highest in a subset of activated/regulatory $T$ lymphocytes

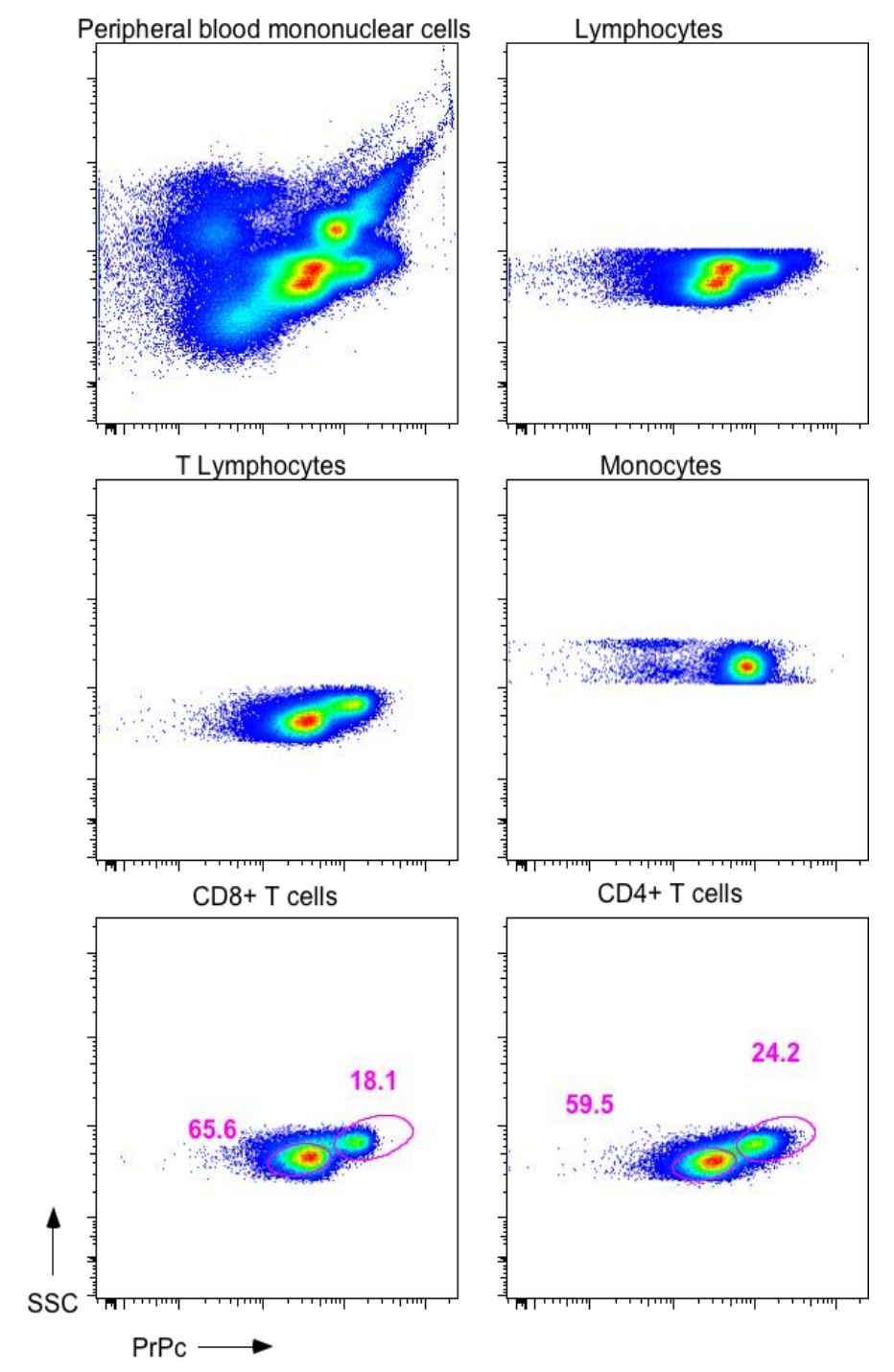

Ex vivo healthy donor PBMCs were surface stained and acquired for FACS analysis. 
EX VIVO CD25+ T CELLS EXPRESS HIGHER LEVELS OF CELLULAR PRION PROTEIN

Figure 56: Ex vivo CD25+ $T$ cells express higher levels of cellular prion protein.

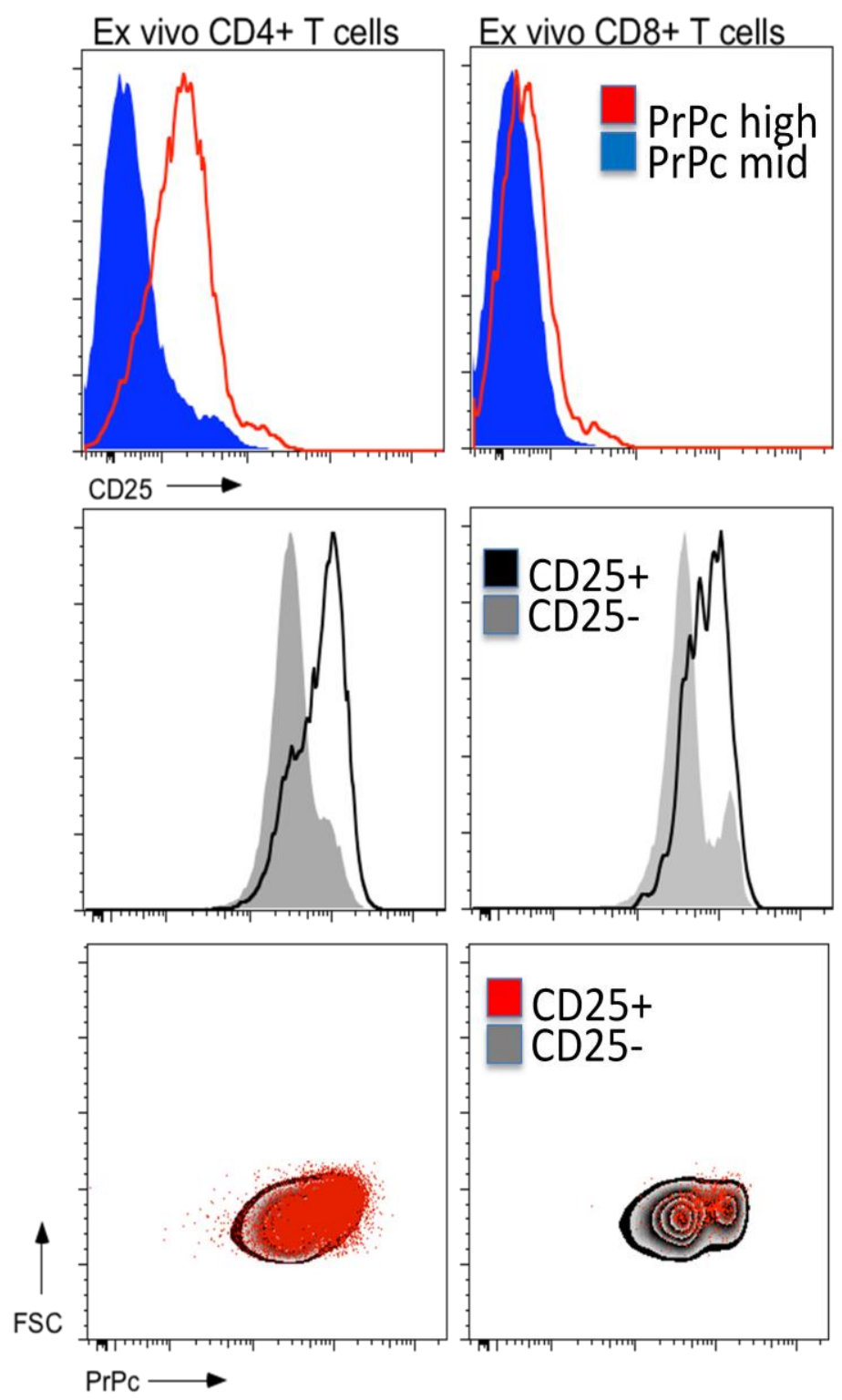

Ex vivo healthy donor PBMCs were surface stained and acquired for FACS analysis. 
AFTER ACTIVATION, FOXP3+ CD4+CD25+ $\mathrm{T}$ CELLS EXPRESSED HIGHER LEVEL OF PRION PROTEIN

Figure 57: After activation, Foxp3+ CD4+CD25+ $T$ cells expressed higher level of Prion Protein
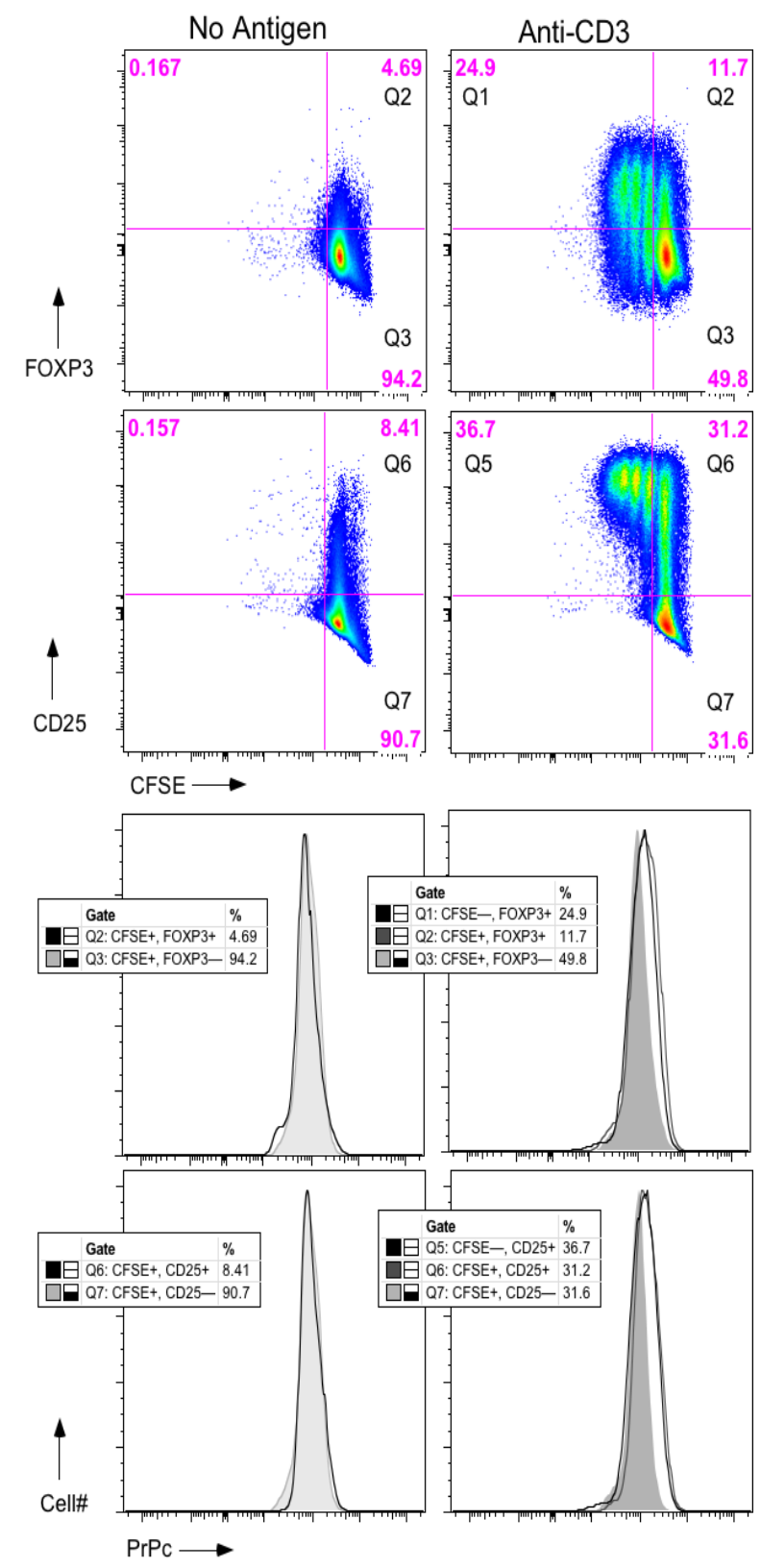

Healthy donor PBMCs were stimulated with anti-CD3 for 7 days and surface stained and acquired for FACS analysis. 


\section{THE ROLE OF T CELLS IN TRANSPLANTATION}

\section{IMMUNOLOGY OF ALLOGRAFT REJECTION/TOLERANCE}

The contributions of direct and indirect recognition to allograft survival or acute and chronic rejection remain unclear. Direct antigen presentation involves donor APC presenting donor MHC peptides. Indirect antigen presentation involves recipient $\mathrm{APC}$ presenting donor $\mathrm{MHC}$ peptides. The indirect pathway may prolong allograft survival. The direct pathway is thought to mediate acute rejection and the indirect perhaps modulates chronic rejection [353]. However, in heart transplant, indirect alloantigen presentation to $\mathrm{CD} 8+\mathrm{T}$ cells results in a bystander, non-pathogenic phenotype [354]. Thus, CD8+ T cells which recognized donor peptide in the context of host MHC were not participatory during acute graft rejection. Indirect antigen presentation, but not direct presentation, results in persistent $\mathrm{T}$ cell trafficking to lymph nodes, but not the spleen [355]. This suggests that indirect antigen presentation and the lymph node microenvironment are necessary for alloantigen-specific tolerization by CD8+ Tregs, potentially in contrast to the theory that CD8+ Tregs arise naturally and are competent to suppress centrally from the thymus. The context of priming by antigen presenting cells plays a critical role in the alloantigen-specific $\mathrm{T}$ cell response $[356,357]$. Suppressor CD8+CD11b+ T cells can be generated in vitro through culturing naïve CD8+ $\mathrm{T}$ cells with alloantigen-primed CD4+ T cells [358]. Presumably, indirect alloantigen presentation in vivo after bone marrow transplant results in similar suppressor $\mathrm{CD} 8+$ cells, which down regulate immunoglobulin production [359]. 


\section{STEM CELL TRANSPLANT}

Hematopoietic stem cell transplant is used to treat hematologic malignancies. Allogeneic transplant exhibits an enhanced graft-versus-tumor effect, but this is accompanied by significant morbidity and mortality due to graft-versus-host disease (GvHD). In GvHD, donor T cells attack recipient epithelia the intestine, skin, and liver. Autologous transplant displays diminished graft-versus-tumor and -host phenomena, but relapses occur more frequently. Treatment of recipients with an anti-CD25 monoclonal antibody enhances allogeneic stem cell engraftment and expands alloantigen-specific regulatory T cells [360]. Blockade of the CD8 molecules, as well, inhibits down-regulation of alloantigen-specific immune responses [361]. Several other studies have shown a positive relationship between increased Treg frequency and reduced incidence of GvHD [362-364]. IL-10 induction of Tregs or production by regulatory T cells is thought to prolong graft acceptance [365-367].

\section{UMBILICAL CORD BLOOD}

A major source of multipotent hematopoietic stem cells (HSC) is umbilical cord blood (UCB). Cord blood HSC, in contrast to embryonic stem cells, are termed adult or fetal stem cells. HSC may give rise to more HSC and all formed elements in the blood. HSC are required to engraft in hematopoietic tissue transplants into patients who have undergone purposeful ablation of their hematopoietic organ, reducing the burden of occult malignant cells and resident $\mathrm{T}$ cells. HSC purification from UCB is accomplished through positive selection for CD34 and Thy-1 and negative selection for other lineages (CD10, CD14, CD15, 
CD16, CD19, and CD20) [368]. Isolation of HSC is required to purge grafts of unwanted populations from clinically functional and transplantable HSC population. Immune reconstitution following a HSC transplant presents a risk for opportunistic infection. CD4+ T lymphocyte recovery takes between 3 and 6 months to reach $>100 \mathrm{CD} 4+\mathrm{T}$ cells/ $\mu \mathrm{L}$ [369]. Relative to autologous HSC, allogeneic HSC without T cells eliminates the clinical issue of GVHD and the basic requirement for immunosuppression. The benefit of GVT effect is also eliminated without $\mathrm{T}$ cells. Allogeneic immune reconstitution is likely to be delayed and engraftment failure may occur. With $\mathrm{T}$ cells depletion, the incidence of allogeneic HSC transplant engraftment failure increases. Bone marrow-derived non-HSC populations play a critical role in enhancing engraftment. The engraftment facilitating capacity of the non-HSC population was concentrated in the CD8+ compartment $[370,371]$. Both CD8+ T lymphocytes and CD8+ dendritic cells were shown to facilitate HSC engraphment. Taking advantage of CD8+ cell dose, GVT may be restored without GVHD [372, 373].

UCB HSC transplant (UCB-HSCT) was first performed in 1990 on a 6-year-old boy with Fanconi anemia [374]. UCB HSC is now performed for a variety of clinically severe hematologic, immunologic, and neoplastic disorders. UCB banks, both private and public, are increasing in capacity. A HIV positive patient with newly diagnosed with acute myeloid leukemia (AML) showed long-term control of both HIV and AML after a stem-cell transplant with CD34+ peripheral HSC from a CCR5 deficient donor [375]. Compared to peripheral HSC transplant, UCB-HSCT allows more HLA disparity with less GVHD [376]. The 
mechanism of increased UCB-HSCT may involve diminished CD8+ T cell cytotoxicity [377]. The diminished CD8+ cytotoxicity may potentially involve a more naïve $\mathrm{T}$ lymphocyte repertoire, expressing CD45RA, and more dominant immune regulatory cells, expressing FOXP3, that may both control GVHD [378]. The high proportion of CD45RA+ $\mathrm{T}$ cells is owed to the fact that the fetus in utero is exposed to low levels of environmental pathogens and vaccines compared to adults. UCB CD45RA $+\mathrm{T}$ cells produce less IFN $\gamma$ than adult $\mathrm{CD} 4+\mathrm{T}$ cells after stimulation with alloantigen. UCB CD4+ T cells may produce less IFN $\gamma$ as a result of impaired master regulator transcription factors, such as T-BET, NFAT, AP-1, and phosphorylated STAT4 [379]. Defective antigen-presenting cells in UCB, expressing less costimulatory molecules and $\mathrm{TNF} \alpha$, may impair $\mathrm{T}$ cell differentiation into Th1 cells [380]. Immature dendritic cells (DC) express less costimulatory molecules and promote antigen-specific tolerance [381]. DCs may drive tolerance through the generation of $\mathrm{FOXP}+\mathrm{CD} 25+\mathrm{CD} 4+\mathrm{T}$ regulatory cells [382]. Immune regulatory cells may play a dominant role over IFN $\gamma$ production though, as IFN $\gamma$ plays a protective effect against GVHD [383]. The fetus in utero is exposed to higher macrophage colony stimulating factor (M-CSF) [384] and low inflammatory signals, which may help to promote Th2 differentiation and immature dendritic cells [385] capable of producing more IL10 and less IL12 [386]. Immune regulatory cell populations in UCB may potentially have a role in transplantation tolerance.

The tolerogenic nature of T cells is important in UCB HSC transplant. GVHD, rejection, and reconstitution delay are all immunologic parameters and may 
potentially be managed taking into consideration the regulatory phenotype and function of particular subsets of $\mathrm{T}$ cells. A master regulator transcription factor, FOXP3, is the most specific marker for regulatory $T$ cells (Tregs) [387]. FOXP3+ cells co-express CD25 on the surface [388]. Subsets of Tregs also express CTLA4, GITR, OX40, CD62L, and CCR7 [389-393].

\section{THE ROLE OF CD8+ TREGS IN TRANSPLANT}

Regulatory CD8+ T cells are selectively resistant to general immunosuppressant agents, such as Cyclosporin A, allowing them to dominantly down-regulate lymphocyte proliferation [394]. Kidney transplant recipients treated with alemtuzumab have shown a homeostatic replenishment of regulatory CD8+ T cells [395]. Alloreactive CD8+CD103+ regulatory CD8+ T cells are expanded by rapamycin [49, 396]. Sphingosine-1-phosphate receptor modulation plays an important role in mediating CD8+ T cell responses in skin graft rejection [397]. Orthoclone OKT3 is a monoclonal antibody used to prevent rejection in heart, liver, pancreas, and kidney transplant [398-402], and OKT3 is also used to treat T1DM. OKT3 has been demonstrated to selectively expand CD8+ $\mathrm{T}$ cell populations relative to $\mathrm{CD} 4+\mathrm{T}$ cells and induce regulatory $\mathrm{CD} 8+\mathrm{T}$ cells in vivo [299]. Experimentally, pre-transplant cell-based therapy can be accomplished either by infusion with whole cells or MHC-derived synthetic peptides. Donorspecific transfusion, along with anti-CD2, -CD3, and -CD40L monoclonal antibodies, has successfully tolerized heart recipients as long as $\mathrm{T}$ cells home to the lymph node [403]. Alloantigen-specific CD8+CD103+ regulatory T cells are generated in vitro using mixed lymphocyte reactions containing mDCs plus 
TFGbeta [404]. Regulatory CD8+ T cells are protective in liver [39, 43, 50], heart [38], and kidney [42, 297, 405] transplants. CD8+ T cells are required for cardiac allograft survival mediated by ICOS-B7 blockade [53]. Anti-ICOS monoclonal antibodies induce regulatory CD8+PD1+ $\mathrm{T}$ cells, which transfer protection to CD8-deficient allograft recipients. Regulatory CD8+ T cells have been explored as potential agents in cell-based therapies for GvHD [406, 407] following allogeneic bone marrow transplant.

\section{ALLOANTIGEN-SPECIFIC CD8+ TREGS}

In various experimental models, both CD4+ and CD8+ Tregs exhibit alloantigenspecific suppressive ability [408]. Oral allo-antigen exposure induces $\gamma \delta$ CD $8+T$ cells to produce IL-10 and tolerize recipients toward kidney allograft [297]. Panstimulated CD8+CD122+ T cells via anti-CD3/28 suppress allogeneic-T cell IFN $\gamma$ production in a CD28-, CD80-, and CD86-dependent manner, but independently of CTLA-4, PD-1, or ICOS [300]. Alloantigen-specific CD8+PD-1+regulatory T cells are induced by anti-ICOS mAb to produce IL-4 and IFN $\gamma$ suppress alloantigen-specific CD4+ $\mathrm{T}$ cell in cardiac allograft transplant [53]. Donorspecific transfusion, which can be used to tolerize recipients toward allogeneic liver transplantion, induces CD8+CD28- T cells capable of adoptively transferring reduced acute rejection rates to secondary recipients [296]. While less than 5\% of CD8+ express CD103, which binds E-cadherin, CD8+CD103+ T cells potently suppress mixed lymphocyte reactions [49]. Alloantigen-specific CD8+ Treg generated ex vivo from CD8+CD25(-) cells can be induced from CD40-activated B cells [57]. 


\section{IMMUNOLOGY OF ALLOGRAFT REJECTION/TOLERANCE}

The contributions of direct and indirect recognition to allograft survival or acute and chronic rejection are unclear. Direct antigen presentation involves donor APC presenting donor MHC peptides. Indirect antigen presentation involves recipient APC presenting donor MHC peptides. Whether the indirect pathway initiates or enhances rejection is not understood. The direct pathway is thought to mediate acute rejection and the indirect perhaps mediates chronic rejection [353]. However, in heart transplant, indirect alloantigen presentation to CD8+ $\mathrm{T}$ cells results in a bystander, non-pathogenic phenotype [354]. Thus, CD8+ T cells which recognized donor peptide in the context of host MHC were not participatory during acute graft rejection. Indirect antigen presentation, but not direct presentation, results in persistent $\mathrm{T}$ cell trafficking to lymph nodes, but not the spleen [355]. These results suggest that indirect antigen presentation and the lymph node microenvironment are necessary for alloantigen-specific tolerization. The context of priming by antigen presenting cells plays a critical role in the alloantigen-specific T cell response [356, 357]. Suppressor CD8+CD11b+ T cells can be generated in vitro through culturing naïve $\mathrm{CD} 8+\mathrm{T}$ cells with alloantigenprimed CD4+ T cells [37]. Presumably, indirect alloantigen presentation in vivo after bone marrow transplant results in similar suppressor CD8+ cells, which down regulate immunoglobulin production [359]. 
Figure 58: Alloantigen-presentation pathways

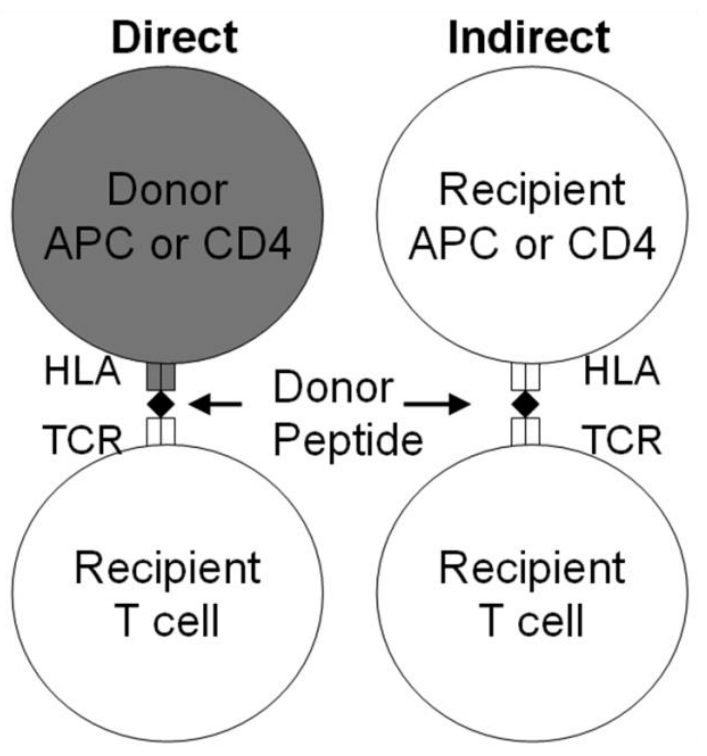


CD8+ TREG SUPPRESS AUTOLOGOUS AND ALLOGENEIC ALLOREACTIVE CD4+CD25(-) T CELLS IN THE BOTH DIRECT AND INDIRECT ANTIGEN PRESENTATION PATHWAYS

Suppression of alloreactive CD4+ T cell stimulation was carried out in culture with varying CD4 to suppressor ratios including the following: 1:0.125, 1:0.25, 1:0.5, and 1:1. CD8 suppressors autologous to CD4 responders or the APC were used, as well as a third party donor. Donor origin is annotated in Figure 10 and Tables 1 and 2 as A, B, and C, respectively. Similar to the previously described flow-based suppression assay, on day 7, cells are stained with florescent antibodies and fixed with $1 \%$ paraformaldehyde. Suppression was determined by comparison to CD4 response in the absence of suppressors. 
Figure 59: Alloantigen-specific CD8+ Treg suppress autologous and allogeneic alloreactive CD4+CD25(-) $\mathrm{T}$ cells in the both direct and indirect antigen presentation pathways.

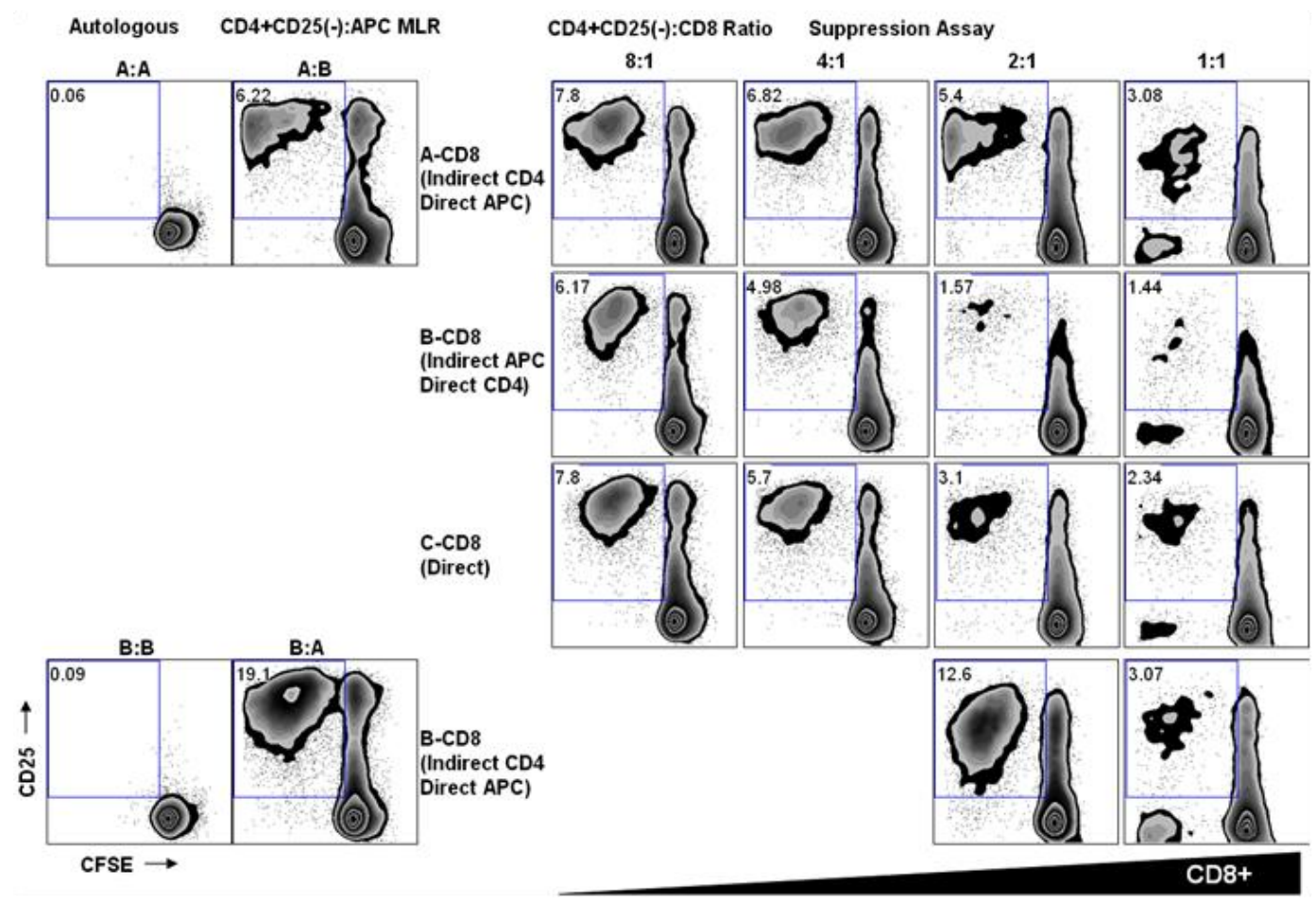

Table 2: Figure 59 Legend

\begin{tabular}{|c|c|c|c|c|}
\hline $\begin{array}{l}\text { Autologous No Stim } \\
\text { CD4+CD25(-):Irrad. } \\
\text { APC }\end{array}$ & $\begin{array}{l}\text { Ex Vivo MLR } \\
\text { CD4+CD25(-) } \rightarrow \text { Irrad. } \\
\text { APC }\end{array}$ & $\begin{array}{l}\text { MLR + Ex Vivo } \\
\text { Suppressors }\end{array}$ & $\begin{array}{l}\text { Expected Result } \\
\text { on CD4 }\end{array}$ & $\begin{array}{l}\text { Expected Result } \\
\text { on APC }\end{array}$ \\
\hline \multirow{9}{*}{ S } & \multirow{9}{*}{$\mathbf{A} \rightarrow \mathbf{B}$} & A-CD8 (Recipient) & $\begin{array}{l}\text { Indirect } \\
\text { Suppression }\end{array}$ & $\begin{array}{l}\text { Direct } \\
\text { Suppression }\end{array}$ \\
\hline & & B CD8 (Donor) & $\begin{array}{l}\text { Direct } \\
\text { Suppression }\end{array}$ & $\begin{array}{l}\text { Indirect } \\
\text { Suppression }\end{array}$ \\
\hline & & $\begin{array}{lrl}\text { C } & \text { CD8 } & \text { (Third } \\
\text { Party) }\end{array}$ & $\begin{array}{l}\text { Direct } \\
\text { Suppression }\end{array}$ & $\begin{array}{l}\text { Direct } \\
\text { Suppression }\end{array}$ \\
\hline & & A CD8+CD28+ & $\begin{array}{l}\text { Indirect } \\
\text { Suppression }\end{array}$ & Direct Suppression \\
\hline & & B CD8+CD28+ & Direct Suppression & $\begin{array}{l}\text { Indirect } \\
\text { Suppression }\end{array}$ \\
\hline & & C CD8+CD28+ & Direct Suppression & Direct Suppression \\
\hline & & A CD8+CD28(-) & $\begin{array}{l}\text { Indirect } \\
\text { Suppression }\end{array}$ & Direct Suppression \\
\hline & & B CD8+CD28(-) & Direct Suppression & $\begin{array}{l}\text { Indirect } \\
\text { Suppression }\end{array}$ \\
\hline & & C CD8+CD28(-) & Direct Suppression & Direct Suppression \\
\hline
\end{tabular}

Ex vivo PBMC from 3 adults (notated A, B and C) were sorted into bulk CD8+,

CD4+CD25(-), and APC. CD4+CD25(-) were cultured with allogeneic APC, and CD8+ T cell suppressive ability was compared among the adult donors. Different donor origin is annotated as $\mathrm{A}, \mathrm{B}$, and $\mathrm{C}$. 
Figure 60: MS patients have deficient CD4 and CD8 Treg function
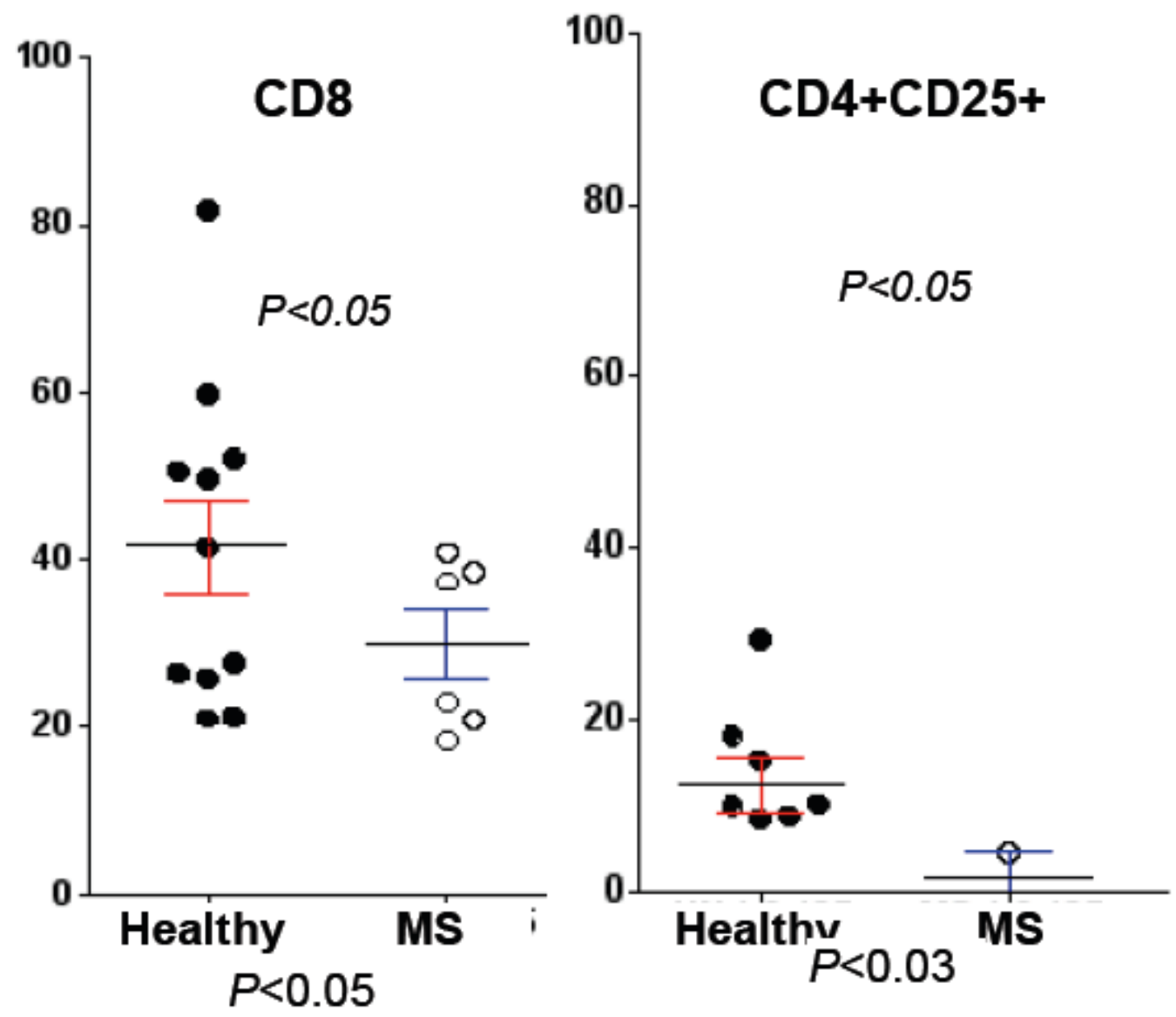

Purified bulk CD8+ T cells (left panel), CD4+CD25high T cells (right panel) or CD4+CD25neg T cells (not shown) were labeled with CMTPX and used in flow cytometry-based suppression assays (similar to those shown in Figure 15). Autologous, CFSE-labeled CD4+CD25neg $\mathrm{T}$ cells were used as responders (stimulated with autologous T celldepleted APC and anti-CD3). Increasing ratios of Tregs were added (data shown here are from a Treg:responder ratio of 0.125:1 in each case). Percent suppression was calculated based on the proliferation of responders alone (without Tregs) and is represented on the $\mathrm{Y}$-axis. 


\section{APPENDIX II: PERSONAL RECORD}

\section{CHRONOLOGY OF FRUITFUL COLLABORATIONS}

While the published narrative of my and my collaborators contribution to biology may seem like an ordered stream, the who and how it all happened was really less well-organized, more serendipitous, mostly having to do with me being in the right place at the right time around so many other smart, diligent people. After I was accepted to the $\mathrm{MD} / \mathrm{PhD}$ program at UT Southwestern Medical Center as an undergraduate, I requested an appointment with Nitin Karandikar (then an associate professor of immunology, pathology, and neurology) in March of 2005. I had read a paper entitled, "Intrathecal synthesis of oligoclonal IgM against myelin lipids predicts an aggressive disease course in MS," regarding antibodies in the CSF of MS patients [409]. It was not a particularly high impact article, but I knew something about lipids and was interested in immunology. Nitin discussed the paper out of consideration for me even though he is more interested in $T$ cells than B cells, and he was kind to hand me a copy of one of his recent publications about myelin-specific CD8+ T cells in MS [202]. I read several of Nitin' publications and met with Nitin again during my first year of medical school to discuss doing my second rotation in his laboratory.

During my rotation in Nitin's laboratory in the summer of 2006, I was trained and supervised daily by Sterling Ortega (then the lab manager/senior technician), who later became a graduate student in our lab. Sterling taught me to run flow cytometry and supervised me acquire a 9 color experiment of CNS-specific CD4+ and CD8+ $\mathrm{T}$ cell responses using the BD LSRII. Sterling taught me digital 
compensation and proper techniques in flow cytometry. In May, I helped Vinodh Pillai (then a senior graduate student) and Sterling do quantitative real time PCR on CD4+CD25+ cells for FOXP3, IL2, IL4, IL10, IFN $\gamma$, TGF $\beta$ and $\beta$ actin as a control. In July, Sterling and Vinodh taught me to intracellularly stain cells for FOXP3. I also helped Vinodh to edit his paper manuscript, "Transient regulatory T-cells: a state attained by all activated human T-cells." After the summer, I returned to second year medical school. Vinodh and Nitin were kind to acknowledge me in their paper when it was published later that winter [91]. Nitin, Vinodh, and Sterling were excellent mentors, and I hoped that they would accept me into the laboratory.

When I returned to Nitin's laboratory in June of 2007 for my "third rotation," Nitin carved out a more-or-less independent project for me that would follow in the footsteps of a previous post-doc, Deepani [104]. In our discussions, Nitin asked me to design an experiment so that we could measure and observe the suppressive ability of CNS-specific CD8+ T cells. This proved to be one of the most pivotal conversations we ever had. After reading several papers using suppression assays, on July 2, I conducted my first 6 day flow-based suppression assay after having tinkered with CMPTX staining of the CD8+ suppressor cells as a strategy for gating them out in the analysis. On July 10, the 7 day flow-based suppression assay was standardized, after having determined the appropriate CFSE-stained responder to CMTXP-stained suppressor ratios and attempting to adapt acquisition to the HTS on the LSR. By the end of July, the suppression assay was validated with CD4+CD25+ cells as a positive control for suppressive 
ability and CD4+CD25- cells as a negative control. Vinodh (then a post-doc) and I began using PKH26 staining of APCs in the suppression assay. Unlike CMPTX and CFSE that make protein adducts, $\mathrm{PKH}$ is a lipophilic proliferation dye that non-colvalently partititions into lipid membranes. The PKH fluorescence was off the scale, and there were problems with toxicity. By August, the simpler CMTPX and CFSE only assay was ideal in my mind, and I began functional studies with healthy human cells in August. After working out all the kinks, I held to this established protocol for the flow-based suppression assay for all the years of my graduate school. My first summer back in the laboratory felt like a success. I was grateful that Vinodh remained in our lab to cross-pollinate my ideas. Thankfully, Nitin agreed to take me into the lab as his newest graduate in the summer of 2007. Now that we had a working suppression assay as a tool, we put it to use with real samples. In the fall of 2007 , I sorted healthy human CD8+ T cells into subsets using CD28 and tested their suppressive ability. By October, I had characterized 11 healthy human donors and began my first experiment with an untreated MS patient with my suppression assay. In November, I generated my first antigenspecific T cell lines after 5 weeks of culture with the help of Larry Anderson (then an assistant professor in internal medicine). I also examined cellular prion protein expression in healthy human PBMC with the collaboration of Olaf Stuve (then an associate professor in neurology).

By the spring semester of 2008, I was assigned to train an undergraduate, Liz Gunter, in collaboration with the UT Dallas Green fellowship program. Promising results led to the first new MS patients recruited from the MS clinic in 
St. Paul, in coloration with Elliot Frohman, professor of neurology. By February, I was able to grow $\mathrm{T}$ cell lines for 10 weeks in culture and conducted suppression assays with transwells. So far, it was clear that healthy and untreated MS patients possessed neuroantigen-specific suppressive ability by CD8+ T cells. Foreignantigen specific $\mathrm{T}$ cell responses were undeterred. In May, we began using cord blood as a source cells, in collaboration with Parkland OBGYN, and induced FOXP3 expression in naïve T cells. Vinodh and I conducted several functional assays together, characterizing FOXP3 in activation-induced CD4+ Tregs. We presented posters of our work at FOCIS in Boston that summer. By the end of the fall semester in 2008 , I had shown that CD8+ T cell could suppress a mixed lymphocyte reaction and had tested if supernatants from CD8+ T cells could inhibit CD4+ T cell responses. By December, I had collected 5 more cord blood donors for functional assays. Vinodh and I spent several weeks writing grants together, and I submitted my first grant to the NIH in December for the F30 individual predoctoral $\mathrm{MD} / \mathrm{PhD}$ fellowship. Nitin helped to hone my scientific writing and presentation skills.

In the spring semester of 2009 , I was co-assigned to train a rotating graduate student, Elizabeth Dimitrova. With the help of Larry, I attempted to use a IFN $\gamma$ ELISPOT assay as a readout of suppression and explored the mechanism of CD8+ T cell suppression with anti-TNFa/HLA class I antibodies. Several sets of $\mathrm{T}$ cell lines were grown and used in dual antigen thymidine-based suppression assays. These experiments helped to answer important questions about the 
specificity and likely HLA restriction of the suppressive neuroantigen-specific CD8+ T cells.

Having had considerable success, Nitin and I decided to move into riskier and bolder strategies with the help of Todd Eagar (then an assistant professor in neurology). In the summer of 2009, Vinodh and I attempted knockdown strategies with lentivirus vectors in primary $\mathrm{T}$ cell lines. Vinodh wanted to knockdown FOXP3, and I aimed for perforin and granzyme B. Vinodh ultimately settled on purchasing lentivirus particles from Santacruz while I toiled to culture plasmid-transfected HEK293T cells with fugene and lipofectamine, culture bacteria, conduct Maxipreps, concentrate virus, infect $\mathrm{T}$ cells with polybrene, and select them with puromycin. It was an excellent learning experience, but not a great source of published data. After Vinodh left the lab to start his residency, I tried my best to show that FOXP3 was required or was not sufficient for functional suppressive ability. Ultimately, I failed. My grant scored mediocre, and I prepared to resubmit a revision in August. Nitin encouraged me to get back up and stick to my guns.

In the fall semester of 2009 , I was assigned to train another rotating graduate student, Chris Peña. I resumed conducting suppression assays with several untreated MS patients and my first acute exacerbation of MS patient in October. Nitin and I experimented with human tonsil immune cells, in collaboration with the Children's Hospital, and I continued mechanistic experiments with a panel of antibodies toward molecules thought to have some effect in my suppression assays. I wrote another grant proposal with Nitin's help in December. That fall 
semester, the suppression assays from several MS patients began to suggest that MS patients during an acute exacerbation were fundamentally different from quiescent MS patients in my CNS-specific CD8 suppression assay. We ramped up patient recruitment, in collaboration with Ben Greenberg (then associate professor of neurology). Jason Mendoza (then a post-doctoral fellow) was also critical in the recruitment of MS patients in the fall 2009 and spring 2010 semesters. Nitin and I began work on the manuscript entitled, "Neuroantigenspecific CD8+ regulatory $\mathrm{T}$-cell function is deficient during acute exacerbation of multiple sclerosis" that spring 2010 semester. I gave a talk and presented posters at FOCIS in Boston with work done in collaboration with Jason and Vinodh, (then a pathology intern at MGH in Boston) that June. Nitin and I spent several months revising and re-drafting my paper for publication in several scientific journals, and it ultimately was published on January 11, 2011 in the Journal of Autoimmunity [2]. The continued effort has focused on the question of the mechanism of CNS-specific suppression. My experience in graduate school continues to be diverse and rich with collaboration due to many gifted scientists. The richness was not only in the questions answered but also in the relationships forged. The scientists of this institution were world-class in terms of their unfettered curiosity and ambitious contributions to human health and biology. Many other important people were critical to the work, as I have included only a few in consideration of brevity. I look forward to continuing to work in such a collaborative environment where training and mentorship are so customary and highly valued. 


\section{REFERENCES}

1. Mills, K.W., et al., Using enzyme-linked immunosorbent assay to detect Escherichia coli K88 pili antigens from clinical isolates. American journal of veterinary research, 1982. 43(2): p. 365-7.

2. Baughman, E.J., et al., Neuroantigen-specific CD8+ regulatory T-cell function is deficient during acute exacerbation of multiple sclerosis. Journal of autoimmunity, 2011.36(2): p. 115-24.

3. Buseman, C.M., et al., Wounding stimulates the accumulation of glycerolipids containing oxophytodienoic acid and dinor-oxophytodienoic acid in Arabidopsis leaves. Plant physiology, 2006. 142(1): p. 28-39.

4. Devaiah, S.P., et al., Quantitative profiling of polar glycerolipid species from organs of wild-type Arabidopsis and a phospholipase Dalpha1 knockout mutant. Phytochemistry, 2006. 67(17): p. 1907-24.

5. Kriegel, M.A., et al., Defective suppressor function of human CD4+ CD25+ regulatory $T$ cells in autoimmune polyglandular syndrome type II. J Exp Med, 2004. 199(9): p. 1285-91.

6. Bonelli, M., J.S. Smolen, and C. Scheinecker, Treg and lupus. Ann Rheum Dis, 2010. 69 Suppl 1: p. i65-66.

7. $\quad$ Noel R. Rose, I.R.M., The autoimmune diseases. Fourth ed2006.

8. Sakaguchi, S., et al., Organ-specific autoimmune diseases induced in mice by elimination of $T$ cell subset. I. Evidence for the active participation of $T$ cells in natural self-tolerance; deficit of a T cell subset as a possible cause of autoimmune disease. J Exp Med, 1985. 161(1): p. 72-87.

9. Furtado, G.C., et al., Interleukin 2 signaling is required for $\mathrm{CD} 4(+)$ regulatory $T$ cell function. The Journal of experimental medicine, 2002. 196(6): p. 851-7.

10. Fontenot, J.D., M.A. Gavin, and A.Y. Rudensky, Foxp3 programs the development and function of $C D 4+C D 25+$ regulatory $T$ cells. Nature immunology, 2003. 4(4): p. 330-6.

11. Kobayashi, S., et al., Beta 2-microglobulin-deficient background ameliorates lethal phenotype of the TGF-beta 1 null mouse. Journal of Immunology, 1999. 163(7): p. 4013-9.

12. Asano, M., et al., Autoimmune disease as a consequence of developmental abnormality of a T cell subpopulation. J Exp Med, 1996. 184(2): p. 38796.

13. Sakaguchi, S., Naturally arising Foxp3-expressing CD25+CD4+ regulatory $T$ cells in immunological tolerance to self and non-self. Nat Immunol, 2005. 6(4): p. 345-52.

14. Sakaguchi, S., et al., Naturally arising Foxp3-expressing CD25+CD4+ regulatory $T$ cells in self-tolerance and autoimmune disease. Curr Top Microbiol Immunol, 2006. 305: p. 51-66. 
15. Baecher-Allan, C., E. Wolf, and D.A. Hafler, Functional analysis of highly defined, FACS-isolated populations of human regulatory CD4+ CD25+ T cells. Clin Immunol, 2005. 115(1): p. 10-8.

16. Lawson, J.M., et al., Increased resistance to $C D 4+C D 25$ hi regulatory $T$ cell-mediated suppression in patients with type 1 diabetes. Clin Exp Immunol, 2008. 154(3): p. 353-9.

17. Tang, Q., et al., In vitro-expanded antigen-specific regulatory $T$ cells suppress autoimmune diabetes. J Exp Med, 2004. 199(11): p. 1455-65.

18. Jaeckel, E., H. von Boehmer, and M.P. Manns, Antigen-specific FoxP3transduced T-cells can control established type 1 diabetes. Diabetes, 2005. 54(2): p. 306-10.

19. Battaglia, M., et al., Induction of tolerance in type 1 diabetes via both CD4+CD25+ $T$ regulatory cells and $T$ regulatory type 1 cells. Diabetes, 2006. 55(6): p. 1571-80.

20. Battaglia, M., et al., Rapamycin and interleukin-10 treatment induces $T$ regulatory type 1 cells that mediate antigen-specific transplantation tolerance. Diabetes, 2006. 55(1): p. 40-9.

21. Tarbell, K.V., et al., Dendritic cell-expanded, islet-specific CD4+CD25+ $C D 62 L+$ regulatory $T$ cells restore normoglycemia in diabetic NOD mice. J Exp Med, 2007. 204(1): p. 191-201.

22. Powell, B.R., N.R. Buist, and P. Stenzel, An X-linked syndrome of diarrhea, polyendocrinopathy, and fatal infection in infancy. J Pediatr, 1982. 100(5): p. 731-7.

23. Wijchers, P.J., J.P. Burbach, and M.P. Smidt, In control of biology: of mice, men and Foxes. Biochem J, 2006. 397(2): p. 233-46.

24. Gershon, R.K., et al., Suppressor T cells. J Immunol, 1972. 108(3): p. 58690.

25. Penhale, W.J., et al., Spontaneous thyroiditis in thymectomized and irradiated Wistar rats. Clinical and Experimental Immunology, 1973. 15(2): p. 225-36.

26. Nishizuka, Y. and T. Sakakura, Thymus and reproduction: sex-linked dysgenesia of the gonad after neonatal thymectomy in mice. Science, 1969. 166(906): p. 753-5.

27. Smith, H., et al., Effector and regulatory cells in autoimmune oophoritis elicited by neonatal thymectomy. Journal of Immunology, 1991. 147(9): p. 2928-33.

28. Sakaguchi, S., T. Takahashi, and Y. Nishizuka, Study on cellular events in postthymectomy autoimmune oophoritis in mice. I. Requirement of Lyt-1 effector cells for oocytes damage after adoptive transfer. The Journal of experimental medicine, 1982. 156(6): p. 1565-76.

29. Penhale, W.J., et al., Thyroiditis in T cell-depleted rats: suppression of the autoallergic response by reconstitution with normal lymphoid cells. Clinical and Experimental Immunology, 1976. 25(1): p. 6-16.

30. Miller, A., et al., Suppressor $T$ cells generated by oral tolerization to myelin basic protein suppress both in vitro and in vivo immune responses by the release of transforming growth factor beta after antigen-specific 
triggering. Proceedings of the National Academy of Sciences of the United States of America, 1992. 89(1): p. 421-5.

31. Inoue, T., et al., Distinction of mouse CD8+ suppressor effector $T$ cell clones from cytotoxic $T$ cell clones by cytokine production and CD45 isoforms. Journal of Immunology, 1993. 150(6): p. 2121-8.

32. Munro, A., The I-J paradox remains unsolved. Nature, 1983. 306(5943): p. 537-8.

33. Athanassakis, I. and S. Vassiliadis, T-regulatory cells: are we rediscovering T suppressors? Immunology letters, 2002. 84(3): p. 179-83.

34. Little, C.C. and E.E. Tyzzer, Further experimental studies on the inheritance of susceptibility to a Transplantable tumor, Carcinoma (J. W. A.) of the Japanese waltzing Mouse. The Journal of medical research, 1916. 33(3): p. 393-453.

35. Schutze, H., P.A. Gorer, and M.H. Finlayson, The Resistance of Four Mouse Lines to Bacterial Infection. The Journal of hygiene, 1936. 36(1): p. 37-49.

36. Kronenberg, M., et al., RNA transcripts for I-J polypeptides are apparently not encoded between the I-A and I-E subregions of the murine major histocompatibility complex. Proceedings of the National Academy of Sciences of the United States of America, 1983. 80(18): p. 5704-8.

37. Koide, J. and E.G. Engleman, Differences in surface phenotype and mechanism of action between alloantigen-specific CD8+ cytotoxic and suppressor T cell clones. J Immunol, 1990. 144(1): p. 32-40.

38. Colovai, A.I., et al., Regulatory CD8+CD28- T cells in heart transplant recipients. Hum Immunol, 2003. 64(1): p. 31-7.

39. Sindhi, R., et al., Reduced immunosuppression in pediatric liver-intestine transplant recipients with CD8+CD28- T-suppressor cells. Hum Immunol, 2005. 66(3): p. 252-7.

40. Suciu-Foca, N., et al., Molecular characterization of allospecific $T$ suppressor and tolerogenic dendritic cells: review. Int Immunopharmacol, 2005. 5(1): p. 7-11.

41. Filaci, G., et al., CD8+CD28- $T$ regulatory lymphocytes inhibiting $T$ cell proliferative and cytotoxic functions infiltrate human cancers. J Immunol, 2007. 179(7): p. 4323-34.

42. Trzonkowski, P., et al., Homeostatic repopulation by CD28-CD8+T cells in alemtuzumab-depleted kidney transplant recipients treated with reduced immunosuppression. Am J Transplant, 2008. 8(2): p. 338-47.

43. Lin, Y.X., et al., Analysis of CD8+CD28- T-suppressor cells in living donor liver transplant recipients. Hepatobiliary Pancreat Dis Int, 2009. 8(3): p. 241-6.

44. Sarantopoulos, S., L. Lu, and H. Cantor, Qa-1 restriction of CD8+ suppressor T cells. J Clin Invest, 2004. 114(9): p. 1218-21.

45. Peng, G., et al., Tumor-infiltrating gammadelta $T$ cells suppress $T$ and dendritic cell function via mechanisms controlled by a unique toll-like receptor signaling pathway. Immunity, 2007. 27(2): p. 334-48. 
46. Mahic, M., et al., Generation of highly suppressive adaptive CD8(+)CD25(+)FOXP3(+) regulatory $T$ cells by continuous antigen stimulation. Eur J Immunol, 2008. 38(3): p. 640-6.

47. Lee, Y.H., et al., Essential role of $C D 8+C D 122+$ regulatory $T$ cells in the recovery from experimental autoimmune encephalomyelitis. J Immunol, 2008. 180(2): p. 825-32.

48. Suzuki, H., et al., Are CD8+CD122+ cells regulatory $T$ cells or memory $T$ cells? Hum Immunol, 2008. 69(11): p. 751-4.

49. Uss, E., et al., CD103 is a marker for alloantigen-induced regulatory CD8+ T cells. J Immunol, 2006. 177(5): p. 2775-83.

50. Lu, L., et al., CD8(+)CD103(+) regulatory $T$ cells in spontaneous tolerance of liver allografts. Int Immunopharmacol, 2009. 9(5): p. 546-8.

51. Kim, J., et al., Conversion of alloantigen-specific CD8+ $T$ cell anergy to CD8+ $T$ cell priming through in vivo ligation of glucocorticoid-induced TNF receptor. Journal of Immunology, 2006. 176(9): p. 5223-31.

52. Kim, J., et al., Prevention of chronic graft-versus-host disease by stimulation with glucocorticoid-induced TNF receptor. Experimental \& molecular medicine, 2006. 38(1): p. 94-9.

53. Izawa, A., et al., A novel alloantigen-specific $C D 8+P D 1+$ regulatory $T$ cell induced by ICOS-B7h blockade in vivo. J Immunol, 2007. 179(2): p. 786-96.

54. Manavalan, J.S., et al., Alloantigen specific CD8+CD28- FOXP3+ T suppressor cells induce ILT3+ ILT4+ tolerogenic endothelial cells, inhibiting alloreactivity. Int Immunol, 2004. 16(8): p. 1055-68.

55. Meloni, F., et al., Foxp3 expressing CD4+CD25+ and CD8+CD28- T regulatory cells in the peripheral blood of patients with lung cancer and pleural mesothelioma. Hum Immunol, 2006. 67(1-2): p. 1-12.

56. Kiniwa, Y., et al., CD8+ Foxp3+ regulatory $T$ cells mediate immunosuppression in prostate cancer. Clin Cancer Res, 2007. 13(23): p. 6947-58.

57. Zheng, J., et al., Efficient induction and expansion of human alloantigenspecific CD8 regulatory $T$ cells from naive precursors by $C D 40$-activated B cells. J Immunol, 2009. 183(6): p. 3742-50.

58. Kang, H.K., M. Liu, and S.K. Datta, Low-dose peptide tolerance therapy of lupus generates plasmacytoid dendritic cells that cause expansion of autoantigen-specific regulatory $T$ cells and contraction of inflammatory Th17 cells. J Immunol, 2007. 178(12): p. 7849-58.

59. Jiang, H., The Qa-1 dependent CD8+T cell mediated regulatory pathway. Cell Mol Immunol, 2005. 2(3): p. 161-7.

60. Niederkorn, J.Y., Emerging concepts in CD8(+) T regulatory cells. Curr Opin Immunol, 2008.

61. Chattopadhyay, S., J. O'Rourke, and R.E. Cone, Implication for the CD94/NKG2A-Qa-1 system in the generation and function of ocularinduced splenic CD8+ regulatory $T$ cells. Int Immunol, 2008. 20(4): p. 509-16. 
62. Sugita, S., et al., B7+ iris pigment epithelium induce CD8+T regulatory cells; both suppress CTLA-4+ T cells. J Immunol, 2006. 176(1): p. 11827.

63. Cone, R.E., et al., The suppression of delayed-type hypersensitivity by CD8+ regulatory T cells requires interferon-gamma. Immunology, 2007. 120(1): p. 112-9.

64. Keino, H., et al., CD8+ T regulatory cells use a novel genetic program that includes CD103 to suppress Th1 immunity in eye-derived tolerance. Invest Ophthalmol Vis Sci, 2006. 47(4): p. 1533-42.

65. Gonzalez-Rey, E., et al., Vasoactive intestinal peptide generates human tolerogenic dendritic cells that induce CD4 and CD8 regulatory $T$ cells. Blood, 2006. 107(9): p. 3632-8.

66. Jarvis, L.B., et al., Autoreactive human peripheral blood CD8+T cells with a regulatory phenotype and function. Eur J Immunol, 2005. 35(10): p. 2896-908.

67. Correale, J. and A. Villa, Isolation and characterization of CD8+ regulatory $T$ cells in multiple sclerosis. J Neuroimmunol, 2008. 195(1-2): p. 121-34.

68. James, E.A. and W.W. Kwok, CD8+ suppressor-mediated regulation of human $\mathrm{CD} 4+T$ cell responses to glutamic acid decarboxylase 65 . Eur $\mathrm{J}$ Immunol, 2007. 37(1): p. 78-86.

69. Bluestone, J.A. and Q. Tang, Therapeutic vaccination using CD4+CD25+ antigen-specific regulatory T cells. Proc Natl Acad Sci U S A, 2004. 101 Suppl 2: p. 14622-6.

70. Filaci, G., et al., Non-antigen-specific CD8(+) T suppressor lymphocytes in diseases characterized by chronic immune responses and inflammation. Ann N Y Acad Sci, 2005. 1050: p. 115-23.

71. Bienvenu, B., et al., Peripheral CD8+CD25+ T lymphocytes from $M H C$ class II-deficient mice exhibit regulatory activity. J Immunol, 2005. 175(1): p. 246-53.

72. Metwali, A., et al., Induction of $C D 8+$ regulatory $T$ cells in the intestine by Heligmosomoides polygyrus infection. Am J Physiol Gastrointest Liver Physiol, 2006. 291(2): p. G253-9.

73. Allez, M., et al., Expansion of CD8+ T cells with regulatory function after interaction with intestinal epithelial cells. Gastroenterology, 2002. 123(5): p. 1516-26.

74. Hayashi, T., et al., 3-Hydroxyanthranilic acid inhibits PDK1 activation and suppresses experimental asthma by inducing $T$ cell apoptosis. Proceedings of the National Academy of Sciences of the United States of America, 2007. 104(47): p. 18619-24.

75. Schmitz, M.L. and D. Krappmann, Controlling NF-kappaB activation in $T$ cells by costimulatory receptors. Cell death and differentiation, 2006. 13(5): p. 834-42.

76. Murphy, K.M. and S.L. Reiner, The lineage decisions of helper T cells. Nature reviews. Immunology, 2002. 2(12): p. 933-44. 
77. Szabo, S.J., et al., A novel transcription factor, T-bet, directs Th1 lineage commitment. Cell, 2000. 100(6): p. 655-69.

78. Ho, I.C., D. Lo, and L.H. Glimcher, c-maf promotes Thelper cell type 2 (Th2) and attenuates Th1 differentiation by both interleukin 4-dependent and -independent mechanisms. The Journal of experimental medicine, 1998. 188(10): p. 1859-66.

79. Zheng, W. and R.A. Flavell, The transcription factor GATA-3 is necessary and sufficient for Th2 cytokine gene expression in CD4 T cells. Cell, 1997. 89(4): p. 587-96.

80. Weaver, C.T., et al., IL-17 family cytokines and the expanding diversity of effector T cell lineages. Annual review of immunology, 2007. 25: p. 82152.

81. Bettelli, E., T. Korn, and V.K. Kuchroo, Th17: the third member of the effector $T$ cell trilogy. Current opinion in immunology, 2007. 19(6): p. 652-7.

82. Ivanov, II, et al., The orphan nuclear receptor RORgammat directs the differentiation program of proinflammatory IL-17+T helper cells. Cell, 2006. 126(6): p. 1121-33.

83. Eberl, G. and D.R. Littman, The role of the nuclear hormone receptor RORgammat in the development of lymph nodes and Peyer's patches. Immunological reviews, 2003. 195: p. 81-90.

84. Hori, S., T. Nomura, and S. Sakaguchi, Control of regulatory $T$ cell development by the transcription factor Foxp3. Science, 2003. 299(5609): p. 1057-61.

85. Chen, W., et al., Conversion of peripheral CD4+CD25-naive T cells to $C D 4+C D 25+$ regulatory $T$ cells by TGF-beta induction of transcription factor Foxp3. The Journal of experimental medicine, 2003. 198(12): p. 1875-86.

86. Gorelik, L., S. Constant, and R.A. Flavell, Mechanism of transforming growth factor beta-induced inhibition of $T$ helper type 1 differentiation. The Journal of experimental medicine, 2002. 195(11): p. 1499-505.

87. Bettelli, E., et al., Reciprocal developmental pathways for the generation of pathogenic effector TH17 and regulatory T cells. Nature, 2006. 441(7090): p. 235-8.

88. Zhou, L., et al., IL-6 programs $T(H)-17$ cell differentiation by promoting sequential engagement of the IL-21 and IL-23 pathways. Nature immunology, 2007. 8(9): p. 967-74.

89. Veldhoen, M., et al., TGFbeta in the context of an inflammatory cytokine milieu supports de novo differentiation of IL-17-producing $T$ cells. Immunity, 2006. 24(2): p. 179-89.

90. Joosten, S.A., et al., Identification of a human CD8+ regulatory $T$ cell subset that mediates suppression through the chemokine CC chemokine ligand 4. Proc Natl Acad Sci U S A, 2007. 104(19): p. 8029-34.

91. Pillai, V., et al., Transient regulatory T-cells: a state attained by all activated human T-cells. Clin Immunol, 2007. 123(1): p. 18-29. 
92. McFarland, H.F. and R. Martin, Multiple sclerosis: a complicated picture of autoimmunity. Nat Immunol, 2007. 8(9): p. 913-9.

93. Antel, J.P., et al., Activated suppressor cell dysfunction in progressive multiple sclerosis. J Immunol, 1986. 137(1): p. 137-41.

94. Jurewicz, A., W.E. Biddison, and J.P. Antel, MHC class I-restricted lysis of human oligodendrocytes by myelin basic protein peptide-specific CD 8 T lymphocytes. J Immunol, 1998. 160(6): p. 3056-9.

95. Babbe, H., et al., Clonal expansions of CD8(+) T cells dominate the $T$ cell infiltrate in active multiple sclerosis lesions as shown by micromanipulation and single cell polymerase chain reaction. J Exp Med, 2000. 192(3): p. 393-404.

96. Huseby, E.S., et al., A pathogenic role for myelin-specific CD8(+) T cells in a model for multiple sclerosis. J Exp Med, 2001. 194(5): p. 669-76.

97. Jacobsen, M., et al., Oligoclonal expansion of memory CD8+ $T$ cells in cerebrospinal fluid from multiple sclerosis patients. Brain, 2002. 125(Pt 3): p. 538-50.

98. Arbour, N., et al., A new approach for evaluating antigen-specific $T$ cell responses to myelin antigens during the course of multiple sclerosis. $\mathrm{J}$ Neuroimmunol, 2003. 137(1-2): p. 197-209.

99. Chess, L. and H. Jiang, Resurrecting CD8+ suppressor T cells. Nat Immunol, 2004. 5(5): p. 469-71.

100. Crawford, M.P., et al., High Prevalence of Autoreactive NeuroantigenSpecific CD8+ T Cells in Multiple Sclerosis Revealed by Novel Flow Cytometric Assay. Blood, 2004. 103: p. 4222-4231.

101. Hu, D., et al., Analysis of regulatory CD8 T cells in Qa-1-deficient mice. Nat Immunol, 2004. 5(5): p. 516-23.

102. Skulina, C., et al., Multiple sclerosis: brain-infiltrating CD8+T cells persist as clonal expansions in the cerebrospinal fluid and blood. Proc Natl Acad Sci U S A, 2004. 101(8): p. 2428-33.

103. Niland, B., et al., CD8+ $T$ cell-mediated HLA-A*0201-restricted cytotoxicity to transaldolase peptide 168-176 in patients with multiple sclerosis. J Immunol, 2005. 175(12): p. 8365-78.

104. Tennakoon, D.K., et al., Therapeutic induction of regulatory, cytotoxic CD8+ T cells in multiple sclerosis. J Immunol, 2006. 176(11): p. 7119-29.

105. Smith, T.R. and V. Kumar, Revival of CD8+ Treg-mediated suppression. Trends Immunol, 2008. 29(7): p. 337-42.

106. York, N.R., et al., Immune regulatory CNS-reactive CD8+T cells in experimental autoimmune encephalomyelitis. J Autoimmun.

107. Sospedra, M. and R. Martin, Immunology of multiple sclerosis. Annu Rev Immunol, 2005. 23: p. 683-747.

108. Anderson, D.W., et al., Revised estimate of the prevalence of multiple sclerosis in the United States. Ann Neurol, 1992. 31(3): p. 333-6.

109. Hallpike, J.F.A., C. W. M. (Colin Wallace Maitland), Trtellotte, Wallace W., Multiple sclerosis : pathology, diagnosis and management1983: Baltimore : Williams \& Wilkins, c1983. 
110. Traugott, U., E.L. Reinherz, and C.S. Raine, Multiple sclerosis: distribution of $T$ cell subsets within active chronic lesions. Science, 1983. 219(4582): p. 308-10.

111. Booss, J., et al., Immunohistological analysis of T lymphocyte subsets in the central nervous system in chronic progressive multiple sclerosis. $\mathrm{J}$ Neurol Sci, 1983. 62(1-3): p. 219-32.

112. Woodroofe, M.N., et al., Immunocytochemical characterisation of the immune reaction in the central nervous system in multiple sclerosis. Possible role for microglia in lesion growth. J Neurol Sci, 1986. 74(2-3): p. 135-52.

113. Trapp, B.D., et al., Axonal transection in the lesions of multiple sclerosis. The New England journal of medicine, 1998. 338(5): p. 278-85.

114. Fontana, A., W. Fierz, and H. Wekerle, Astrocytes present myelin basic protein to encephalitogenic T-cell lines. Nature, 1984. 307(5948): p. 2736.

115. Klawiter, E.C., et al., Radial diffusivity predicts demyelination in ex vivo multiple sclerosis spinal cords. NeuroImage, 2011. 55(4): p. 1454-60.

116. Bitsch, A. and W. Bruck, Differentiation of multiple sclerosis subtypes: implications for treatment. CNS Drugs, 2002. 16(6): p. 405-18.

117. Delmotte P., G.R.E., Immunological and Clinical Aspects of Multiple Sclerosis1984, Hingham, MA: MTP Press.

118. Lin VW, C.D., Cutter NC, et al., editors., Spinal Cord Medicine: Principles and Practice2003, New York: Demos Medical Publishing.

119. McDonnell, G.V. and S.A. Hawkins, Primary progressive multiple sclerosis: a distinct syndrome? Mult Scler, 1996. 2(3): p. 137-41.

120. [Diagnostics and drug therapy of multiple sclerosis]. Duodecim, 2002. 118(13): p. 1411-23.

121. Brinkman, C.J., W.M. Nillesen, and O.R. Hommes, T-cell subpopulations in blood and cerebrospinal fluid of multiple sclerosis patients: effect of cyclophosphamide. Clin Immunol Immunopathol, 1983. 29(3): p. 341-8.

122. Hauser, S.L., et al., CSF cells in multiple sclerosis: monoclonal antibody analysis and relationship to peripheral blood T-cell subsets. Neurology, 1983. 33(5): p. 575-9.

123. Poser, C.M., et al., New diagnostic criteria for multiple sclerosis: guidelines for research protocols. Ann Neurol, 1983. 13(3): p. 227-31.

124. Sawcer, S. and P.N. Goodfellow, Inheritance of susceptibility to multiple sclerosis. Current opinion in immunology, 1998. 10(6): p. 697-703.

125. Biddison, W.E., et al., Distinct epitopes on the T8 molecule are differentially involved in cytotoxic T cell function. Hum Immunol, 1984. 9(2): p. 117-30.

126. Antel, J., et al., Defective suppressor cell function mediated by T8+ cell lines from patients with progressive multiple sclerosis. J Immunol, 1986. 137(11): p. 3436-9.

127. Peltonen, L., Old suspects found guilty--the first genome profile of multiple sclerosis. N Engl J Med, 2007. 357(9): p. 927-9. 
128. Keegan, B.M. and J.H. Noseworthy, Multiple sclerosis. Annu Rev Med, 2002. 53: p. 285-302.

129. Wallin, M.T., W.F. Page, and J.F. Kurtzke, Multiple sclerosis in US veterans of the Vietnam era and later military service: race, sex, and geography. Annals of neurology, 2004. 55(1): p. 65-71.

130. O'Connor, K.C., et al., Myelin basic protein-reactive autoantibodies in the serum and cerebrospinal fluid of multiple sclerosis patients are characterized by low-affinity interactions. J Neuroimmunol, 2003. 136(12): p. 140-8.

131. Piyasirisilp, S., T. Hemachudha, and D.E. Griffin, B-cell responses to myelin basic protein and its epitopes in autoimmune encephalomyelitis induced by Semple rabies vaccine. J Neuroimmunol, 1999. 98(2): p. 96104.

132. Rivers, T.M. and F.F. Schwentker, Encephalomyelitis Accompanied by Myelin Destruction Experimentally Produced in Monkeys. J Exp Med, 1935. 61(5): p. 689-702.

133. Arnason, B.G., Relevance of experimental allergic encephalomyelitis to multiple sclerosis. Neurol Clin, 1983. 1(3): p. 765-82.

134. Pohl-Koppe, A., et al., Myelin basic protein reactive Th2 T cells are found in acute disseminated encephalomyelitis. Journal of neuroimmunology, 1998. 91(1-2): p. 19-27.

135. O'Connor, K.C., et al., Self-antigen tetramers discriminate between myelin autoantibodies to native or denatured protein. Nature medicine, 2007. 13(2): p. 211-7.

136. Tuohy, V.K., et al., Identification of an encephalitogenic determinant of myelin proteolipid protein for SJL mice. Journal of Immunology, 1989. 142(5): p. 1523-7.

137. Amor, S., et al., Encephalitogenic epitopes of myelin basic protein, proteolipid protein, myelin oligodendrocyte glycoprotein for experimental allergic encephalomyelitis induction in Biozzi ABH (H-2Ag7) mice share an amino acid motif. Journal of Immunology, 1996. 156(8): p. 3000-8.

138. Stromnes, I.M. and J.M. Goverman, Passive induction of experimental allergic encephalomyelitis. Nature protocols, 2006. 1(4): p. 1952-60.

139. Stromnes, I.M. and J.M. Goverman, Active induction of experimental allergic encephalomyelitis. Nature protocols, 2006. 1(4): p. 1810-9.

140. Sun, D., et al., Myelin antigen-specific CD8+ T cells are encephalitogenic and produce severe disease in C57BL/6 mice. Journal of Immunology, 2001. 166(12): p. 7579-87.

141. Ford, M.L. and B.D. Evavold, Specificity, magnitude, and kinetics of $M O G$-specific CD8+ $T$ cell responses during experimental autoimmune encephalomyelitis. European Journal of Immunology, 2005. 35(1): p. 7685.

142. Huseby, E.S., C. Ohlen, and J. Goverman, Cutting edge: myelin basic protein-specific cytotoxic $T$ cell tolerance is maintained in vivo by a single dominant epitope in H-2k mice. Journal of Immunology, 1999. 163(3): p. 1115-8. 
143. Fazekas, G. and T. Tabira, What transgenic and knockout mouse models teach us about experimental autoimmune encephalomyelitis. Reviews in immunogenetics, 2000. 2(1): p. 115-32.

144. Linker, R.A., et al., CNTF is a major protective factor in demyelinating CNS disease: a neurotrophic cytokine as modulator in neuroinflammation. Nature medicine, 2002. 8(6): p. 620-4.

145. Farias, A.S., et al., Nitric oxide and TNFalpha effects in experimental autoimmune encephalomyelitis demyelination. Neuroimmunomodulation, 2007. 14(1): p. 32-8.

146. Malipiero, U., et al., Myelin oligodendrocyte glycoprotein-induced autoimmune encephalomyelitis is chronic/relapsing in perforin knockout mice, but monophasic in Fas- and Fas ligand-deficient lpr and gld mice. European Journal of Immunology, 1997. 27(12): p. 3151-60.

147. Berghmans, N., et al., Interferon-gamma Orchestrates the Number and Function of Th17 Cells in Experimental Autoimmune Encephalomyelitis. Journal of interferon \& cytokine research : the official journal of the International Society for Interferon and Cytokine Research, 2011.

148. Willenborg, D.O., et al., IFN-gamma is critical to the control of murine autoimmune encephalomyelitis and regulates both in the periphery and in the target tissue: a possible role for nitric oxide. Journal of Immunology, 1999. 163(10): p. 5278-86.

149. Guo, B., E.Y. Chang, and G. Cheng, The type I IFN induction pathway constrains Th17-mediated autoimmune inflammation in mice. The Journal of clinical investigation, 2008. 118(5): p. 1680-90.

150. Bettelli, E., et al., IL-10 is critical in the regulation of autoimmune encephalomyelitis as demonstrated by studies of IL-10- and IL-4-deficient and transgenic mice. Journal of Immunology, 1998. 161(7): p. 3299-306.

151. Gomez-Nicola, D., et al., Aggravated experimental autoimmune encephalomyelitis in IL-15 knockout mice. Experimental neurology, 2010. 222(2): p. 235-42.

152. Wu, X., et al., Cerebral interleukin-15 shows upregulation and beneficial effects in experimental autoimmune encephalomyelitis. Journal of neuroimmunology, 2010. 223(1-2): p. 65-72.

153. Diveu, C., et al., IL-27 blocks RORc expression to inhibit lineage commitment of Th17 cells. Journal of Immunology, 2009. 182(9): p. 574856.

154. Matsushita, T., et al., Regulatory B cells (B10 cells) and regulatory $T$ cells have independent roles in controlling experimental autoimmune encephalomyelitis initiation and late-phase immunopathogenesis. Journal of Immunology, 2010. 185(4): p. 2240-52.

155. Abdul-Majid, K.B., et al., Comparing the pathogenesis of experimental autoimmune encephalomyelitis in CD4-/- and CD8-/- DBA/1 mice defines qualitative roles of different $T$ cell subsets. Journal of neuroimmunology, 2003. 141(1-2): p. 10-9. 
156. Koh, D.R., et al., Less mortality but more relapses in experimental allergic encephalomyelitis in CD8-/- mice. Science, 1992. 256(5060): p. 1210-3.

157. Linker, R.A., et al., EAE in beta-2 microglobulin-deficient mice: axonal damage is not dependent on MHC-I restricted immune responses. Neurobiology of disease, 2005. 19(1-2): p. 218-28.

158. Hu, D., et al., Analysis of regulatory CD8 T cells in Qa-1-deficient mice. Nature immunology, 2004. 5(5): p. 516-23.

159. Teige, A., et al., CD1-dependent regulation of chronic central nervous system inflammation in experimental autoimmune encephalomyelitis. Journal of Immunology, 2004. 172(1): p. 186-94.

160. Gallon, L., et al., Differential effects of B7-1 blockade in the rat experimental autoimmune encephalomyelitis model. Journal of Immunology, 1997. 159(9): p. 4212-6.

161. Verhagen, J., et al., Enhanced selection of FoxP3+ T-regulatory cells protects CTLA-4-deficient mice from CNS autoimmune disease. Proceedings of the National Academy of Sciences of the United States of America, 2009. 106(9): p. 3306-11.

162. Salama, A.D., et al., Critical role of the programmed death-1 (PD-1) pathway in regulation of experimental autoimmune encephalomyelitis. The Journal of experimental medicine, 2003. 198(1): p. 71-8.

163. Jee, Y., et al., STAT expression and localization in the central nervous system during autoimmune encephalomyelitis in Lewis rats. Journal of neuroimmunology, 2001. 114(1-2): p. 40-7.

164. Chitnis, T., et al., Effect of targeted disruption of STAT4 and STAT6 on the induction of experimental autoimmune encephalomyelitis. The Journal of clinical investigation, 2001. 108(5): p. 739-47.

165. Waldner, H., et al., Fas- and FasL-deficient mice are resistant to induction of autoimmune encephalomyelitis. Journal of Immunology, 1997. 159(7): p. 3100-3.

166. Li, Q., et al., Endothelial IL-1RI is a critical mediator of EAE pathogenesis. Brain, behavior, and immunity, 2011. 25(1): p. 160-7.

167. Duong, T.T., et al., Effect of anti-interferon-gamma and anti-interleukin-2 monoclonal antibody treatment on the development of actively and passively induced experimental allergic encephalomyelitis in the SJL/J mouse. Journal of neuroimmunology, 1992. 36(2-3): p. 105-15.

168. Okuda, Y., et al., IL-6 plays a crucial role in the induction phase of myelin oligodendrocyte glucoprotein 35-55 induced experimental autoimmune encephalomyelitis. Journal of neuroimmunology, 1999. 101(2): p. 188-96.

169. Korn, T., et al., IL-6 controls Th17 immunity in vivo by inhibiting the conversion of conventional $T$ cells into Foxp3+ regulatory $T$ cells. Proceedings of the National Academy of Sciences of the United States of America, 2008. 105(47): p. 18460-5.

170. Walline, C.C., S. Kanakasabai, and J.J. Bright, IL-7Ralpha confers susceptibility to experimental autoimmune encephalomyelitis. Genes and immunity, 2011. 12(1): p. 1-14. 
171. Becher, B., B.G. Durell, and R.J. Noelle, Experimental autoimmune encephalitis and inflammation in the absence of interleukin-12. The Journal of clinical investigation, 2002. 110(4): p. 493-7.

172. Komiyama, Y., et al., IL-17 plays an important role in the development of experimental autoimmune encephalomyelitis. Journal of Immunology, 2006. 177(1): p. 566-73.

173. Wang, G.Y., et al., IL-17 eliminates the therapeutic effects of myelin basic protein-induced nasal tolerance in experimental autoimmune encephalomyelitis by activating IL-6. Scandinavian journal of immunology, 2008. 68(6): p. 589-97.

174. Touil, T., et al., Pathophysiology of interleukin-23 in experimental autoimmune encephalomyelitis. Drug news \& perspectives, 2006. 19(2): p. 77-83.

175. Illes, Z., et al., Modulation of CD4 co-receptor limits spontaneous autoimmunity when high-affinity transgenic TCR specific for self-antigen is expressed on a genetically resistant background. International immunology, 2007. 19(10): p. 1235-48.

176. Cheng, S., et al., Characterization of HLA DR2 and DQ8 transgenic mouse with a new engineered mouse class II deletion, which lacks all endogenous class II genes. Journal of autoimmunity, 2003. 21(3): p. 1959.

177. Girvin, A.M., et al., A critical role for B7/CD28 costimulation in experimental autoimmune encephalomyelitis: a comparative study using costimulatory molecule-deficient mice and monoclonal antibody blockade. Journal of Immunology, 2000. 164(1): p. 136-43.

178. Chang, T.T., et al., Studies in B7-deficient mice reveal a critical role for B7 costimulation in both induction and effector phases of experimental autoimmune encephalomyelitis. The Journal of experimental medicine, 1999. 190(5): p. 733-40.

179. Perrin, P.J., et al., Experimental autoimmune meningitis: a novel neurological disease in CD28-deficient mice. Clinical immunology, 1999. 91(1): p. 41-9.

180. Nath, N., et al., T-bet is essential for the progression of experimental autoimmune encephalomyelitis. Immunology, 2006. 118(3): p. 384-91.

181. Begum-Haque, S., et al., Downregulation of IL-17 and IL-6 in the central nervous system by glatiramer acetate in experimental autoimmune encephalomyelitis. Journal of neuroimmunology, 2008. 204(1-2): p. 58-65.

182. Tada, Y., et al., Reduced incidence and severity of antigen-induced autoimmune diseases in mice lacking interferon regulatory factor-1. The Journal of experimental medicine, 1997. 185(2): p. 231-8.

183. Marta, M., Toll-like receptors in multiple sclerosis mouse experimental models. Annals of the New York Academy of Sciences, 2009. 1173: p. 458-62.

184. Tang, X., et al., Regulation of immunity by a novel population of Qa-1restricted CD8alphaalpha+TCRalphabeta+ $T$ cells. Journal of Immunology, 2006. 177(11): p. 7645-55. 
185. Chen, W., et al., Perceiving the avidity of $T$ cell activation can be translated into peripheral $T$ cell regulation. Proceedings of the National Academy of Sciences of the United States of America, 2007. 104(51): p. 20472-7.

186. Allegretta, M., et al., T cells responsive to myelin basic protein in patients with multiple sclerosis. Science, 1990. 247(4943): p. 718-21.

187. Bielekova, B., et al., Expansion and functional relevance of high-avidity myelin-specific CD4+ $T$ cells in multiple sclerosis. Journal of Immunology, 2004. 172(6): p. 3893-904.

188. Anderson, A.C., et al., High frequency of autoreactive myelin proteolipid protein-specific $T$ cells in the periphery of naive mice: mechanisms of selection of the self-reactive repertoire [see comments]. J Exp Med, 2000. 191(5): p. 761-70.

189. Lang, H.L., et al., A functional and structural basis for TCR crossreactivity in multiple sclerosis. Nature immunology, 2002. 3(10): p. 940-3.

190. Simpson, E., et al., T cells with dual antigen specificity in T cell receptor transgenic mice rejecting allografts. European Journal of Immunology, 1995. 25(10): p. 2813-7.

191. Heath, W.R., et al., Expression of two $T$ cell receptor alpha chains on the surface of normal murine T cells. European Journal of Immunology, 1995. 25(6): p. 1617-23.

192. Martin, R., et al., Myelin basic protein-specific T-cell responses in identical twins discordant or concordant for multiple sclerosis. Annals of neurology, 1993. 34(4): p. 524-35.

193. Rice, G.P., et al., Disease activity markers in multiple sclerosis. Another look at suppressor cells defined by monoclonal antibodies OKT4, OKT5, and OKT8. J Neuroimmunol, 1984. 6(2): p. 75-84.

194. Albala, M.M., et al., Normal T cell subsets and lymphocyte activity in multiple sclerosis. Clin Exp Immunol, 1985. 61(3): p. 542-7.

195. Bach, M.A., et al., T cell subsets in multiple sclerosis. A longitudinal study of exacerbating-remitting cases. J Neuroimmunol, 1985. 7(5-6): p. 33143.

196. Zaffaroni, M., et al., T-cell subsets in multiple sclerosis: relationships between peripheral blood and cerebrospinal fluid. Acta Neurol Scand, 1985. 71(3): p. 242-8.

197. Weiner, H.L., et al., Altered blood T-cell subsets in patients with multiple sclerosis. J Neuroimmunol, 1984. 6(2): p. 115-21.

198. Hafler, D.A., et al., Investigation of in vivo activated T cells in multiple sclerosis and inflammatory central nervous system diseases. Clin Immunol Immunopathol, 1985. 37(2): p. 163-71.

199. Hafler, D.A., et al., Myelin basic protein and proteolipid protein reactivity of brain- and cerebrospinal fluid-derived $T$ cell clones in multiple sclerosis and postinfectious encephalomyelitis. J Immunol, 1987. 139(1): p. 68-72. 
200. Noronha, A., D.P. Richman, and B.G. Arnason, Multiple sclerosis: activated cells in cerebrospinal fluid in acute exacerbations. Ann Neurol, 1985. 18(6): p. 722-5.

201. Stone, L.A., et al., Blood-brain barrier disruption on contrast-enhanced MRI in patients with mild relapsing-remitting multiple sclerosis: relationship to course, gender, and age. Neurology, 1995. 45(6): p. 11226.

202. Crawford, M.P., et al., High prevalence of autoreactive, neuroantigenspecific CD8+ $T$ cells in multiple sclerosis revealed by novel flow cytometric assay. Blood, 2004. 103(11): p. 4222-31.

203. Biegler, B.W., et al., Glatiramer acetate (GA) therapy induces a focused, oligoclonal CD8+ T-cell repertoire in multiple sclerosis. Journal of neuroimmunology, 2006. 180(1-2): p. 159-71.

204. Biegler, B.W., et al., Clonal composition of neuroantigen-specific CD8+ and CD4+ T-cells in multiple sclerosis. Journal of neuroimmunology, 2011.

205. Jacobsen, M., et al., Oligoclonal expansion of memory CD8+ $T$ cells in cerebrospinal fluid from multiple sclerosis patients. Brain : a journal of neurology, 2002. 125(Pt 3): p. 538-50.

206. Striebich, C.C., et al., Selective accumulation of related CD4+ $T$ cell clones in the synovial fluid of patients with rheumatoid arthritis. Journal of Immunology, 1998. 161(8): p. 4428-36.

207. Wu, H.D., et al., The lymphocytic infiltration in calcific aortic stenosis predominantly consists of clonally expanded $T$ cells. Journal of Immunology, 2007. 178(8): p. 5329-39.

208. Li, Y., et al., Local-clonal expansion of infiltrating $T$ lymphocytes in chronic encephalitis of Rasmussen. Journal of Immunology, 1997. 158(3): p. 1428-37.

209. Andersson, P.B., E. Waubant, and D.E. Goodkin, How should we proceed with disease-modifying treatments for multiple sclerosis? Lancet, 1997. 349(9052): p. 586-7.

210. Paty, D.W. and D.K. Li, Interferon beta-1b is effective in relapsingremitting multiple sclerosis. II. MRI analysis results of a multicenter, randomized, double-blind, placebo-controlled trial. UBC MS/MRI Study Group and the IFNB Multiple Sclerosis Study Group. Neurology, 1993. 43(4): p. 662-7.

211. Interferon beta-1b is effective in relapsing-remitting multiple sclerosis. $I$. Clinical results of a multicenter, randomized, double-blind, placebocontrolled trial. The IFNB Multiple Sclerosis Study Group. Neurology, 1993. 43(4): p. 655-61.

212. Randomised double-blind placebo-controlled study of interferon beta-1a in relapsing/remitting multiple sclerosis. PRISMS (Prevention of Relapses and Disability by Interferon beta-1a Subcutaneously in Multiple Sclerosis) Study Group. Lancet, 1998. 352(9139): p. 1498-504.

213. Placebo-controlled multicentre randomised trial of interferon beta-1b in treatment of secondary progressive multiple sclerosis. European Study 
Group on interferon beta-1b in secondary progressive MS. Lancet, 1998. 352(9139): p. 1491-7.

214. Jacobs, L.D., et al., Intramuscular interferon beta-1a for disease progression in relapsing multiple sclerosis. The Multiple Sclerosis Collaborative Research Group (MSCRG). Ann Neurol, 1996. 39(3): p. 285-94.

215. Beck, R.W., et al., Interferon beta-la for early multiple sclerosis: CHAMPS trial subgroup analyses. Ann Neurol, 2002. 51(4): p. 481-90.

216. Byrnes, A.A., J.C. McArthur, and C.L. Karp, Interferon-beta therapy for multiple sclerosis induces reciprocal changes in interleukin-12 and interleukin-10 production. Ann Neurol, 2002. 51(2): p. 165-74.

217. Dayal, A.S., et al., Interferon-gamma-secreting cells in multiple sclerosis patients treated with interferon beta-1b. Neurology, 1995. 45(12): p. 2173-7.

218. Interferon beta-1b in the treatment of multiple sclerosis: final outcome of the randomized controlled trial. The IFNB Multiple Sclerosis Study Group and The University of British Columbia MS/MRI Analysis Group. Neurology, 1995. 45(7): p. 1277-85.

219. Polman, C.H., et al., A randomized, placebo-controlled trial of natalizumab for relapsing multiple sclerosis. N Engl J Med, 2006. 354(9): p. 899-910.

220. Hartung, H.P., et al., Mitoxantrone in progressive multiple sclerosis: a placebo-controlled, double-blind, randomised, multicentre trial. Lancet, 2002. 360(9350): p. 2018-25.

221. Brinkman, C.J., W.M. Nillesen, and O.R. Hommes, The effect of cyclophosphamide on T lymphocytes and T lymphocyte subsets in patients with chronic progressive multiple sclerosis. Acta Neurol Scand, 1984. 69(2): p. 90-6.

222. Frohman, E.M., et al., Corticosteroids for multiple sclerosis: I. Application for treating exacerbations. Neurotherapeutics, 2007. 4(4): $p$. 618-26.

223. Teitelbaum, D., et al., Suppression of experimental allergic encephalomyelitis by a synthetic polypeptide. Eur J Immunol, 1971. 1(4): p. 242-8.

224. Teitelbaum, D., et al., Suppression by several synthetic polypeptides of experimental allergic encephalomyelitis induced in guinea pigs and rabbits with bovine and human basic encephalitogen. Eur J Immunol, 1973. 3(5): p. 273-9.

225. Teitelbaum, D., et al., Suppression of experimental allergic encephalomyelitis in Rhesus monkeys by a synthetic basic copolymer. Clin Immunol Immunopathol, 1974. 3(2): p. 256-62.

226. Abramsky, O., D. Teitelbaum, and R. Arnon, Effect of a synthetic polypeptide (COP 1) on patients with multiple sclerosis and with acute disseminated encephalomeylitis. Preliminary report. J Neurol Sci, 1977. 31(3): p. 433-8. 
227. Keith, A.B., et al., The effect of Cop 1, a synthetic polypeptide, on chronic relapsing experimental allergic encephalomyelitis in guinea pigs. J Neurol Sci, 1979. 42(2): p. 267-74.

228. Glatiramer acetate for relapsing multiple sclerosis. Med Lett Drugs Ther, 1997. 39(1004): p. 61-2.

229. Glatiramer acetate for multiple sclerosis. Drug Ther Bull, 2001. 39(6): p. 41-3.

230. Bornstein, M.B., et al., Multiple sclerosis: trial of a synthetic polypeptide. Ann Neurol, 1982. 11(3): p. 317-9.

231. Schrempf, W. and T. Ziemssen, Glatiramer acetate: mechanisms of action in multiple sclerosis. Autoimmun Rev, 2007. 6(7): p. 469-75.

232. Aharoni, R., et al., Oral treatment of mice with copolymer 1 (glatiramer acetate) results in the accumulation of specific Th2 cells in the central nervous system. J Neuroimmunol, 2002. 126(1-2): p. 58-68.

233. Aktas, O., et al., Multiple sclerosis: modulation of apoptosis susceptibility by glatiramer acetate. Acta Neurol Scand, 2001. 104(5): p. 266-70.

234. Hussien, Y., et al., Glatiramer acetate and IFN-beta act on dendritic cells in multiple sclerosis. J Neuroimmunol, 2001. 121(1-2): p. 102-10.

235. Aharoni, R., et al., Copolymer 1 acts against the immunodominant epitope 82-100 of myelin basic protein by $T$ cell receptor antagonism in addition to major histocompatibility complex blocking. Proc Natl Acad Sci U S A, 1999. 96(2): p. 634-9.

236. Aharoni, R., et al., Copolymer 1 induces $T$ cells of the Thelper type 2 that crossreact with myelin basic protein and suppress experimental autoimmune encephalomyelitis. Proc Natl Acad Sci U S A, 1997. 94(20): p. 10821-6.

237. Kappos, L., et al., Induction of a non-encephalitogenic type 2 Thelper-cell autoimmune response in multiple sclerosis after administration of an altered peptide ligand in a placebo-controlled, randomized phase II trial. The Altered Peptide Ligand in Relapsing MS Study Group. Nature medicine, 2000. 6(10): p. 1176-82.

238. Duda, P.W., et al., Glatiramer acetate (Copaxone) induces degenerate, Th2-polarized immune responses in patients with multiple sclerosis. The Journal of clinical investigation, 2000. 105(7): p. 967-76.

239. Nicholas, M.K., et al., Dissociation of T8+ cell-mediated cytolytic and suppressor functions in young adults. J Clin Lab Immunol, 1986. 20(2): p. 51-5.

240. Kipnis, J., et al., T cell immunity to copolymer 1 confers neuroprotection on the damaged optic nerve: possible therapy for optic neuropathies. Proceedings of the National Academy of Sciences of the United States of America, 2000. 97(13): p. 7446-51.

241. Stuve, O., et al., Immunologic, clinical, and radiologic status 14 months after cessation of natalizumab therapy. Neurology, 2009. 72(5): p. 396401. 
242. Friese, M.A. and L. Fugger, Autoreactive CD8+ T cells in multiple sclerosis: a new target for therapy? Brain : a journal of neurology, 2005. 128(Pt 8): p. 1747-63.

243. Viglietta, V., et al., Loss of functional suppression by $C D 4+C D 25+$ regulatory $T$ cells in patients with multiple sclerosis. J Exp Med, 2004. 199(7): p. 971-9.

244. Haas, J., et al., Reduced suppressive effect of CD4+CD25high regulatory $T$ cells on the $T$ cell immune response against myelin oligodendrocyte glycoprotein in patients with multiple sclerosis. Eur J Immunol, 2005. 35(11): p. 3343-52.

245. Karandikar, N.J., et al., Glatiramer acetate (Copaxone) therapy induces CD8(+) T cell responses in patients with multiple sclerosis. J Clin Invest, 2002. 109(5): p. 641-649.

246. Haas, J., et al., Prevalence of newly generated naive regulatory $T$ cells (Treg) is critical for Treg suppressive function and determines Treg dysfunction in multiple sclerosis. Journal of Immunology, 2007. 179(2): p. 1322-30.

247. Hao, S., et al., Novel exosome-targeted CD4+ $T$ cell vaccine counteracting CD4+25+ regulatory $T$ cell-mediated immune suppression and stimulating efficient central memory CD8+CTL responses. Journal of Immunology, 2007. 179(5): p. 2731-40.

248. Anderson, M.S., et al., Projection of an immunological self shadow within the thymus by the aire protein. Science, 2002. 298(5597): p. 1395-401.

249. Byrne, J.A. and M.B. Oldstone, Biology of cloned cytotoxic T lymphocytes specific for lymphocytic choriomeningitis virus: clearance of virus in vivo. J Virol, 1984. 51(3): p. 682-6.

250. Lukacher, A.E., V.L. Braciale, and T.J. Braciale, In vivo effector function of influenza virus-specific cytotoxic T lymphocyte clones is highly specific. J Exp Med, 1984. 160(3): p. 814-26.

251. Bania, M.B., et al., Suppressor and cytolytic cell function in multiple sclerosis. Effects of cyclosporine A and interleukin 2. J Clin Invest, 1986. 78(2): p. 582-6.

252. Reinherz, E.L., et al., Loss of suppressor $T$ cells in active multiple sclerosis. Analysis with monoclonal antibodies. The New England journal of medicine, 1980. 303(3): p. 125-9.

253. Antel, J.P., B.G. Arnason, and M.E. Medof, Suppressor cell function in multiple sclerosis: correlation with clinical disease activity. Annals of neurology, 1979. 5(4): p. 338-42.

254. Chou, Y.K., et al., Human CD8+ T cell clone regulates autologous CD4+ myelin basic protein specific T cells. Autoimmunity, 1992. 14(2): p. 1119.

255. Jiang, H., S.I. Zhang, and B. Pernis, Role of CD8+ T cells in murine experimental allergic encephalomyelitis. Science, 1992. 256(5060): p. 1213-5.

256. Correale, J., et al., T cell vaccination in secondary progressive multiple sclerosis. Journal of neuroimmunology, 2000. 107(2): p. 130-9. 
257. Vandenbark, A.A., et al., Treatment of multiple sclerosis with T-cell receptor peptides: results of a double-blind pilot trial. Nature medicine, 1996. 2(10): p. 1109-15.

258. Zang, Y.C., et al., Increased CD8+ cytotoxic $T$ cell responses to myelin basic protein in multiple sclerosis. Journal of Immunology, 2004. 172(8): p. 5120-7.

259. Fernando, M.M., et al., Defining the role of the MHC in autoimmunity: a review and pooled analysis. PLoS genetics, 2008. 4(4): p. e1000024.

260. Snell, G.D., Methods for the study of histocompatibility genes. Journal of genetics, 1948. 49(2): p. 87-108.

261. Dausset, J., [Iso-leuko-antibodies]. Acta haematologica, 1958. 20(1-4): p. 156-66.

262. Benacerraf, B., Role of MHC gene products in immune regulation. Science, 1981. 212(4500): p. 1229-38.

263. Bai, A., J. Broen, and J. Forman, The pathway for processing leaderderived peptides that regulate the maturation and expression of Qa-1b. Immunity, 1998. 9(3): p. 413-21.

264. Wei, M.L. and P. Cresswell, HLA-A2 molecules in an antigen-processing mutant cell contain signal sequence-derived peptides. Nature, 1992. 356(6368): p. 443-6.

265. Braud, V.M., et al., TAP- and tapasin-dependent HLA-E surface expression correlates with the binding of an MHC class I leader peptide. Current biology : CB, 1998. 8(1): p. 1-10.

266. Hermel, E., et al., Polymorphism and conservation of the genes encoding Qa1 molecules. Immunogenetics, 2004. 56(9): p. 639-49.

267. Lee, N., et al., HLA-E surface expression depends on binding of TAPdependent peptides derived from certain HLA class I signal sequences. Journal of Immunology, 1998. 160(10): p. 4951-60.

268. van der Ven, K. and C. Ober, HLA-G polymorphisms in African Americans. Journal of Immunology, 1994. 153(12): p. 5628-33.

269. Braud, V.M., et al., HLA-E binds to natural killer cell receptors CD94/NKG2A, B and C. Nature, 1998. 391(6669): p. 795-9.

270. Ljunggren, H.G. and K. Karre, In search of the 'missing self': MHC molecules and NK cell recognition. Immunol Today, 1990. 11(7): p. 23744.

271. Aldrich, C.J., et al., Identification of a Tap-dependent leader peptide recognized by alloreactive $T$ cells specific for a class Ib antigen. Cell, 1994. 79(4): p. 649-58.

272. Michaelsson, J., et al., A signal peptide derived from hsp60 binds HLA-E and interferes with CD94/NKG2A recognition. The Journal of experimental medicine, 2002. 196(11): p. 1403-14.

273. Davies, A., et al., A peptide from heat shock protein 60 is the dominant peptide bound to Qa-1 in the absence of the MHC class Ia leader sequence peptide Qdm. Journal of Immunology, 2003. 170(10): p. 502733. 
274. Jiang, H., et al., HLA-E-restricted regulatory CD8(+) T cells are involved in development and control of human autoimmune type 1 diabetes. The Journal of clinical investigation, 2010. 120(10): p. 3641-50.

275. Oliveira, C.C., et al., The nonpolymorphic MHC Qa-1b mediates CD8+T cell surveillance of antigen-processing defects. The Journal of experimental medicine, 2010. 207(1): p. 207-21.

276. Seaman, M.S., C.R. Wang, and J. Forman, MHC class Ib-restricted CTL provide protection against primary and secondary Listeria monocytogenes infection. Journal of Immunology, 2000. 165(9): p. 5192-201.

277. Cho, H., et al., Nonconventional CD8+ $T$ cell responses to Listeria infection in mice lacking MHC class Ia and H2-M3. Journal of Immunology, 2011. 186(1): p. 489-98.

278. Salerno-Goncalves, R., et al., Identification of a human HLA-E-restricted CD8+ $T$ cell subset in volunteers immunized with Salmonella enterica serovar Typhi strain Ty21a typhoid vaccine. Journal of Immunology, 2004. 173(9): p. 5852-62.

279. Pietra, G., et al., HLA-E-restricted recognition of cytomegalovirus-derived peptides by human CD8+ cytolytic T lymphocytes. Proceedings of the National Academy of Sciences of the United States of America, 2003. 100(19): p. 10896-901.

280. Jiang, S. and R.I. Lechler, CD4+CD25+ regulatory T-cell therapy for allergy, autoimmune disease and transplant rejection. Inflamm Allergy Drug Targets, 2006. 5(4): p. 239-42.

281. Zheng, S.G., et al., Transfer of regulatory $T$ cells generated ex vivo modifies graft rejection through induction of tolerogenic CD4+CD25+ cells in the recipient. Int Immunol, 2006. 18(2): p. 279-89.

282. Masteller, E.L., Q. Tang, and J.A. Bluestone, Antigen-specific regulatory $T$ cells -- ex vivo expansion and therapeutic potential. Semin Immunol, 2006. 18(2): p. 103-10.

283. Pedersen, A.E. and F. Ronchese, CTLA-4 blockade during dendritic cell based booster vaccination influences dendritic cell survival and CTL expansion. Journal of immune based therapies and vaccines, 2007. 5: p. 9.

284. Banchereau, J., et al., Immune and clinical responses in patients with metastatic melanoma to CD34(+) progenitor-derived dendritic cell vaccine. Cancer Res, 2001. 61(17): p. 6451-8.

285. Palucka, A.K., et al., Single injection of CD34+ progenitor-derived dendritic cell vaccine can lead to induction of T-cell immunity in patients with stage IV melanoma. J Immunother, 2003. 26(5): p. 432-9.

286. Paczesny, S., et al., Expansion of melanoma-specific cytolytic CD8+T cell precursors in patients with metastatic melanoma vaccinated with CD34+ progenitor-derived dendritic cells. J Exp Med, 2004. 199(11): p. 1503-11.

287. Wrzesinski, C. and N.P. Restifo, Less is more: lymphodepletion followed by hematopoietic stem cell transplant augments adoptive T-cell-based anti-tumor immunotherapy. Curr Opin Immunol, 2005. 17(2): p. 195-201. 
288. Jenkins, M.K. and R.H. Schwartz, Antigen presentation by chemically modified splenocytes induces antigen-specific T cell unresponsiveness in vitro and in vivo. J Exp Med, 1987. 165(2): p. 302-19.

289. Antony, P.A., et al., CD8+ T cell immunity against a tumor/self-antigen is augmented by CD4+ Thelper cells and hindered by naturally occurring $T$ regulatory cells. J Immunol, 2005. 174(5): p. 2591-601.

290. Trenado, A., et al., Ex vivo-expanded CD4+CD25+ immunoregulatory $T$ cells prevent graft-versus-host-disease by inhibiting activation/differentiation of pathogenic T cells. J Immunol, 2006. 176(2): p. 1266-73.

291. Reddy, M.M. and M.H. Grieco, Quantitative changes in Thelper inducer (CD4+ CD45RA-), $T$ suppressor inducer (CD4+ CD45RA+), T suppressor $(C D 8+C D 11 b+)$, and $T$ cytotoxic $(C D 8+C D 11 b-)$ subsets in human immunodeficiency virus infection. J Clin Lab Anal, 1991. 5(2): p. 96-100.

292. Han, G., et al., Suppressor role of rat CD8+CD45RClow $T$ cells in experimental autoimmune uveitis (EAU). J Neuroimmunol, 2007. 183(12): p. 81-8.

293. Menager-Marcq, I., et al., CD8+CD28-regulatory T lymphocytes prevent experimental inflammatory bowel disease in mice. Gastroenterology, 2006. 131(6): p. 1775-85.

294. Elrefaei, M., et al., Presence of suppressor HIV-specific CD8+T cells is associated with increased PD-1 expression on effector CD8+ $T$ cells. $\mathrm{J}$ Immunol, 2008. 180(11): p. 7757-63.

295. Brimnes, J., et al., Defects in CD8+ regulatory $T$ cells in the lamina propria of patients with inflammatory bowel disease. J Immunol, 2005. 174(9): p. 5814-22.

296. Liu, Y., et al., The protective effect of CD8+CD28-T suppressor cells on the acute rejection responses in rat liver transplantation. Transplant Proc, 2007. 39(10): p. 3396-403.

297. Zhou, J., et al., CD8(+)gammadelta $T$ regulatory cells mediate kidney allograft prolongation after oral exposure to alloantigen. Transpl Int, 2008.

298. Herold, K.C., et al., Anti-CD3 monoclonal antibody in new-onset type 1 diabetes mellitus. The New England journal of medicine, 2002. 346(22): p. 1692-8.

299. Ablamunits, V. and K.C. Herold, Generation and function of human regulatory $C D 8+T$ cells induced by a humanized OKT3 monoclonal antibody hOKT3gammal (Ala-Ala). Hum Immunol, 2008. 69(11): p. 7326.

300. Shi, Z., et al., Importance of CD80/CD86-CD28 interactions in the recognition of target cells by $C D 8+C D 122+$ regulatory $T$ cells. Immunology, 2008. 124(1): p. 121-8.

301. Jiang, H., et al., An affinity/avidity model of peripheral $T$ cell regulation. $\mathrm{J}$ Clin Invest, 2005. 115(2): p. 302-12. 
302. McDonald, W.I., Silberberg, Donald H., Multiple sclerosis1986: London ; Boston : Butterworths, 1986.

303. Yssel, H. and H. Spits, Generation and maintenance of cloned human T cell lines. Curr Protoc Immunol, 2002. Chapter 7: p. Unit 719.

304. Berger, C., et al., Adoptive transfer of effector $C D 8+T$ cells derived from central memory cells establishes persistent $T$ cell memory in primates. $\mathrm{J}$ Clin Invest, 2008. 118(1): p. 294-305.

305. Herzenberg, L.A., et al., Interpreting flow cytometry data: a guide for the perplexed. Nat Immunol, 2006. 7(7): p. 681-5.

306. York, N.R., et al., Immune regulatory CNS-reactive CD8+T cells in experimental autoimmune encephalomyelitis. J Autoimmun, 2010. 35(1): p. 33-44.

307. Venken, K., et al., Compromised CD4+CD25(high) regulatory T-cell function in patients with relapsing-remitting multiple sclerosis is correlated with a reduced frequency of FOXP3-positive cells and reduced FOXP3 expression at the single-cell level. Immunology, 2008. 123(1): p. 79-89.

308. Antel, J., et al., Activated suppressor cell function in multiple sclerosis-clinical correlations. J Neuroimmunol, 1988. 17(4): p. 323-30.

309. Bach, M.A., et al., Deficit of suppressor $T$ cells in active multiple sclerosis. Lancet, 1980. 2(8206): p. 1221-3.

310. Heesen, C., et al., Altered cytokine responses to cognitive stress in multiple sclerosis patients with fatigue. Mult Scler, 2005. 11(1): p. 51-7.

311. Tsai, S., et al., Reversal of autoimmunity by boosting memory-like autoregulatory T cells. Immunity, 2010. 32(4): p. 568-80.

312. Meloni, F., et al., Monocyte chemoattractant protein-1 levels in bronchoalveolar lavage fluid of lung-transplanted patients treated with tacrolimus as rescue treatment for refractory acute rejection. Transplant Proc, 2003. 35(4): p. 1523-6.

313. Joosten, S.A. and T.H. Ottenhoff, Human CD4 and CD8 regulatory $T$ cells in infectious diseases and vaccination. Hum Immunol, 2008. 69(11): p. 760-70.

314. Abbas, A.K. and C.A. Janeway, Jr., Immunology: improving on nature in the twenty-first century. Cell, 2000. 100(1): p. 129-38.

315. Flajnik, M.F. and M. Kasahara, Origin and evolution of the adaptive immune system: genetic events and selective pressures. Nat Rev Genet, 2010. 11(1): p. 47-59.

316. Sun, J., D.J. Earl, and M.W. Deem, Glassy dynamics in the adaptive immune response prevents autoimmune disease. Phys Rev Lett, 2005. 95(14): p. 148104.

317. Selvaraj, R.K. and T.L. Geiger, A kinetic and dynamic analysis of Foxp3 induced in T cells by TGF-beta. J Immunol, 2007. 178(12): p. 7667-77.

318. Tran, D.Q., H. Ramsey, and E.M. Shevach, Induction of FOXP3 expression in naive human $C D 4+F O X P 3-T$ cells by $T$ cell receptor stimulation is TGF\{beta\}-dependent but does not confer a regulatory phenotype. Blood, 2007. 
319. Pillai, V. and N.J. Karandikar, Attack on the clones? Human FOXP3 detection by PCH101, 236A/E7, 206D, and 259D reveals 259D as the outlier with lower sensitivity. Blood, 2008. 111(1): p. 463-4; author reply 464-6.

320. Babar, M.E., et al., Genetic variability at seven codons of the prion protein gene in nine Pakistani sheep breeds. Journal of genetics, 2008. 87(2): p. 187-90.

321. Burthem, J., et al., The normal cellular prion protein is strongly expressed by myeloid dendritic cells. Blood, 2001. 98(13): p. 3733-8.

322. Li, R., et al., The expression and potential function of cellular prion protein in human lymphocytes. Cellular immunology, 2001. 207(1): p. 4958.

323. Shyng, S.L., J.E. Heuser, and D.A. Harris, A glycolipid-anchored prion protein is endocytosed via clathrin-coated pits. The Journal of cell biology, 1994. 125(6): p. 1239-50.

324. Westergard, L., H.M. Christensen, and D.A. Harris, The cellular prion protein $(\operatorname{Pr} P(C))$ : its physiological function and role in disease. Biochimica et biophysica acta, 2007. 1772(6): p. 629-44.

325. Spielhaupter, C. and H.M. Schatzl, PrPC directly interacts with proteins involved in signaling pathways. The Journal of biological chemistry, 2001. 276(48): p. 44604-12.

326. Kim, B.H., et al., The cellular prion protein (PrPC) prevents apoptotic neuronal cell death and mitochondrial dysfunction induced by serum deprivation. Brain research. Molecular brain research, 2004. 124(1): p. 4050 .

327. Bounhar, Y., et al., Prion protein protects human neurons against Baxmediated apoptosis. The Journal of biological chemistry, 2001. 276(42): p. 39145-9.

328. Paitel, E., R. Fahraeus, and F. Checler, Cellular prion protein sensitizes neurons to apoptotic stimuli through Mdm2-regulated and p53-dependent caspase 3-like activation. The Journal of biological chemistry, 2003. 278(12): p. 10061-6.

329. Paitel, E., et al., Overexpression of PrPc triggers caspase 3 activation: potentiation by proteasome inhibitors and blockade by anti-PrP antibodies. Journal of neurochemistry, 2002. 83(5): p. 1208-14.

330. Diarra-Mehrpour, M., et al., Prion protein prevents human breast carcinoma cell line from tumor necrosis factor alpha-induced cell death. Cancer research, 2004. 64(2): p. 719-27.

331. Caetano, F.A., et al., Amyloid-beta oligomers increase the localization of prion protein at the cell surface. Journal of neurochemistry, 2011. 117(3): p. 538-553.

332. Brown, D.R., et al., Normal prion protein has an activity like that of superoxide dismutase. The Biochemical journal, 1999. 344 Pt 1: p. 1-5.

333. Hutter, G., F.L. Heppner, and A. Aguzzi, No superoxide dismutase activity of cellular prion protein in vivo. Biological chemistry, 2003. 384(9): p. 1279-85. 
334. Sakudo, A., et al., Impairment of superoxide dismutase activation by $\mathrm{N}$ terminally truncated prion protein (PrP) in PrP-deficient neuronal cell line. Biochemical and biophysical research communications, 2003. 308(3): p. 660-7.

335. Pammer, J., W. Weninger, and E. Tschachler, Human keratinocytes express cellular prion-related protein in vitro and during inflammatory skin diseases. The American journal of pathology, 1998. 153(5): p. 13538.

336. Konturek, P.C., et al., Helicobacter pylori upregulates prion protein expression in gastric mucosa: a possible link to prion disease. World journal of gastroenterology : WJG, 2005. 11(48): p. 7651-6.

337. Zomosa-Signoret, V., et al., Physiological role of the cellular prion protein. Veterinary research, 2008. 39(4): p. 9.

338. McBride, S.M., Prion protein: a pattern recognition receptor for viral components and uric acid responsible for the induction of innate and adaptive immunity. Medical hypotheses, 2005. 65(3): p. 570-7.

339. Pasupuleti, M., et al., Antimicrobial activity of human prion protein is mediated by its N-terminal region. PLoS One, 2009. 4(10): p. e7358.

340. McMahon, H.E., et al., Cleavage of the amino terminus of the prion protein by reactive oxygen species. The Journal of biological chemistry, 2001. 276(3): p. 2286-91.

341. Andersson, E., et al., Antimicrobial activities of heparin-binding peptides. European journal of biochemistry / FEBS, 2004. 271(6): p. 1219-26.

342. Harris, D.A., Trafficking, turnover and membrane topology of PrP. British medical bulletin, 2003. 66: p. 71-85.

343. Mabbott, N.A., et al., T-lymphocyte activation and the cellular form of the prion protein. Immunology, 1997. 92(2): p. 161-5.

344. Tsutsui, S., et al., Absence of the cellular prion protein exacerbates and prolongs neuroinflammation in experimental autoimmune encephalomyelitis. The American journal of pathology, 2008. 173(4): p. 1029-41.

345. $\mathrm{Hu}, \mathrm{W}$. , et al., Pharmacological prion protein silencing accelerates central nervous system autoimmune disease via T cell receptor signalling. Brain : a journal of neurology, 2010. 133(Pt 2): p. 375-88.

346. Cashman, N.R., et al., Cellular isoform of the scrapie agent protein participates in lymphocyte activation. Cell, 1990. 61(1): p. 185-92.

347. Durig, J., et al., Differential constitutive and activation-dependent expression of prion protein in human peripheral blood leucocytes. British journal of haematology, 2000. 108(3): p. 488-95.

348. Fraser, H., et al., Replication of scrapie in spleens of SCID mice follows reconstitution with wild-type mouse bone marrow. The Journal of general virology, 1996. 77 ( Pt 8): p. 1935-40.

349. Brandner, S., et al., Normal host prion protein $(\mathrm{PrPC})$ is required for scrapie spread within the central nervous system. Proceedings of the National Academy of Sciences of the United States of America, 1996. 93(23): p. 13148-51. 
350. Kingsbury, D.T., et al., Evidence for normal cell-mediated immunity in scrapie-infected mice. Infection and immunity, 1981. 32(3): p. 1176-80.

351. Kitamoto, T., et al., Abnormal isoform of prion protein accumulates in follicular dendritic cells in mice with Creutzfeldt-Jakob disease. Journal of Virology, 1991. 65(11): p. 6292-5.

352. Aucouturier, P., et al., Infected splenic dendritic cells are sufficient for prion transmission to the CNS in mouse scrapie. The Journal of clinical investigation, 2001. 108(5): p. 703-8.

353. Caballero, A., et al., Tolerogenic response: allorecognition pathways. Transpl Immunol, 2006. 17(1): p. 3-6.

354. Valujskikh, A., Q. Zhang, and P.S. Heeger, CD8 T cells specific for a donor-derived, self-restricted transplant antigen are nonpathogenic bystanders after vascularized heart transplantation in mice. J Immunol, 2006. 176(4): p. 2190-6.

355. Ochando, J.C., N.R. Krieger, and J.S. Bromberg, Direct versus indirect allorecognition: Visualization of dendritic cell distribution and interactions during rejection and tolerization. Am J Transplant, 2006. 6(10): p. 2488-96.

356. O'Connell, P.J., et al., Immature and mature CD8alpha+ dendritic cells prolong the survival of vascularized heart allografts. J Immunol, 2002. 168(1): p. 143-54.

357. Ochando, J.C., et al., Alloantigen-presenting plasmacytoid dendritic cells mediate tolerance to vascularized grafts. Nat Immunol, 2006. 7(6): p. 65262.

358. Koide, J. and E.G. Engleman, Differences in surface phenotype and mechanism of action between alloantigen-specific CD8+ cytotoxic and suppressor T cell clones. J Immunol, 1990. 144(1): p. 32-40.

359. Lebeck, L.K., H. Kaizer, and H.M. Gebel, Characterization of peripheral blood CD8/11b cells in bone marrow transplant recipients. III. Subsets of CD8/11b cells differentially regulate immunoglobulin production. Bone Marrow Transplant, 1992. 9(1): p. 35-9.

360. Weng, L., J. Dyson, and F. Dazzi, Low-intensity transplant regimens facilitate recruitment of donor-specific regulatory $T$ cells that promote hematopoietic engraftment. Proc Natl Acad Sci U S A, 2007. 104(20): p. 8415-20.

361. Van Seventer, G.A., et al., Evidence for a regulatory role of the T8 (CD8) antigen in antigen-specific and anti-T3-(CD3)-induced lytic activity of allospecific cytotoxic T lymphocyte clones. Eur J Immunol, 1986. 16(11): p. 1363-71.

362. Miura, Y., et al., Association of Foxp3 regulatory gene expression with graft-versus-host disease. Blood, 2004. 104(7): p. 2187-93.

363. Zorn, E., et al., Reduced frequency of FOXP3+CD4+CD25+ regulatory $T$ cells in patients with chronic graft-versus-host disease. Blood, 2005. 106(8): p. 2903-11. 
364. Rezvani, K., et al., High donor FOXP3-positive regulatory T-cell (Treg) content is associated with a low risk of GVHD following HLA-matched allogeneic SCT. Blood, 2006. 108(4): p. 1291-7.

365. Hara, M., et al., IL-10 is required for regulatory $T$ cells to mediate tolerance to alloantigens in vivo. J Immunol, 2001. 166(6): p. 3789-96.

366. Lin, M.T., et al., Relation of an interleukin-10 promoter polymorphism to graft-versus-host disease and survival after hematopoietic-cell transplantation. N Engl J Med, 2003. 349(23): p. 2201-10.

367. Battaglia, G., et al., Properties of [3H]prazosin-labeled alpha 1adrenergic receptors in rat brain and porcine neurointermediate lobe tissue. Journal of neurochemistry, 1983. 41(2): p. 538-42.

368. Shizuru, J.A., R.S. Negrin, and I.L. Weissman, Hematopoietic stem and progenitor cells: clinical and preclinical regeneration of the hematolymphoid system. Annual review of medicine, 2005. 56: p. 509-38.

369. Negrin, R.S., et al., Transplantation of highly purified CD34+Thy-1+ hematopoietic stem cells in patients with metastatic breast cancer. Biology of blood and marrow transplantation : journal of the American Society for Blood and Marrow Transplantation, 2000. 6(3): p. 262-71.

370. Gandy, K.L., et al., CD8+TCR+ and CD8+TCR-cells in whole bone marrow facilitate the engraftment of hematopoietic stem cells across allogeneic barriers. Immunity, 1999. 11(5): p. 579-90.

371. Martin, P.J., Donor CD8 cells prevent allogeneic marrow graft rejection in mice: potential implications for marrow transplantation in humans. The Journal of experimental medicine, 1993. 178(2): p. 703-12.

372. Verneris, M.R., et al., Engineering hematopoietic grafts: purified allogeneic hematopoietic stem cells plus expanded CD8+NK-T cells in the treatment of lymphoma. Biology of blood and marrow transplantation : journal of the American Society for Blood and Marrow Transplantation, 2001. 7(10): p. 532-42.

373. Ito, M. and J.A. Shizuru, Graft-vs.-lymphoma effect in an allogeneic hematopoietic stem cell transplantation model. Biology of blood and marrow transplantation : journal of the American Society for Blood and Marrow Transplantation, 1999. 5(6): p. 357-68.

374. Gluckman, E., et al., Hematopoietic reconstitution in a patient with Fanconi's anemia by means of umbilical-cord blood from an HLAidentical sibling. The New England journal of medicine, 1989. 321(17): p. 1174-8.

375. Hutter, G., et al., Long-term control of HIV by CCR5 Delta32/Delta32 stem-cell transplantation. The New England journal of medicine, 2009. 360(7): p. 692-8.

376. Barker, J.N., et al., Survival after transplantation of unrelated donor umbilical cord blood is comparable to that of human leukocyte antigenmatched unrelated donor bone marrow: results of a matched-pair analysis. Blood, 2001. 97(10): p. 2957-61. 
377. Risdon, G., et al., Proliferative and cytotoxic responses of human cord blood $T$ lymphocytes following allogeneic stimulation. Cellular immunology, 1994. 154(1): p. 14-24.

378. Reen, D.J., Activation and functional capacity of human neonatal CD4 Tcells. Vaccine, 1998. 16(14-15): p. 1401-8.

379. Kaminski, B.A., et al., Reduced expression of NFAT-associated genes in $U C B$ versus adult $C D 4+T$ lymphocytes during primary stimulation. Blood, 2003. 102(13): p. 4608-17.

380. Drohan, L., et al., Selective developmental defects of cord blood antigenpresenting cell subsets. Human Immunology, 2004. 65(11): p. 1356-69.

381. Toubai, T., et al., Immunization with host-type CD8\{alpha\}+ dendritic cells reduces experimental acute GVHD in an IL-10-dependent manner. Blood, 2010. 115(3): p. 724-35.

382. Yamazaki, S., et al., Dendritic cells expand antigen-specific Foxp3+ CD25+CD4+ regulatory $T$ cells including suppressors of alloreactivity. Immunological reviews, 2006. 212: p. 314-29.

383. Yang, Y.G., et al., Donor-derived interferon gamma is required for inhibition of acute graft-versus-host disease by interleukin 12. The Journal of clinical investigation, 1998. 102(12): p. 2126-35.

384. Saito, S., et al., High serum human macrophage colony-stimulating factor level during pregnancy. International journal of hematology, 1992. 55(3): p. 219-25.

385. Mellor, A.L. and D.H. Munn, Immunology at the maternal-fetal interface: lessons for $T$ cell tolerance and suppression. Annual review of immunology, 2000. 18: p. 367-91.

386. Li, G., Y.J. Kim, and H.E. Broxmeyer, Macrophage colony-stimulating factor drives cord blood monocyte differentiation into IL-10(high)IL12absent dendritic cells with tolerogenic potential. Journal of Immunology, 2005. 174(8): p. 4706-17.

387. Fontenot, J.D., et al., Regulatory $T$ cell lineage specification by the forkhead transcription factor foxp3. Immunity, 2005. 22(3): p. 329-41.

388. Sakaguchi, S., et al., Immunologic self-tolerance maintained by activated $T$ cells expressing IL-2 receptor alpha-chains (CD25). Breakdown of a single mechanism of self-tolerance causes various autoimmune diseases. Journal of Immunology, 1995. 155(3): p. 1151-64.

389. Dieckmann, D., et al., Ex vivo isolation and characterization of CD4(+)CD25(+) $T$ cells with regulatory properties from human blood. The Journal of experimental medicine, 2001. 193(11): p. 1303-10.

390. Shimizu, J., et al., Stimulation of CD25(+)CD4(+) regulatory $T$ cells through GITR breaks immunological self-tolerance. Nature immunology, 2002. 3(2): p. 135-42.

391. McHugh, R.S., et al., CD4(+)CD25(+) immunoregulatory T cells: gene expression analysis reveals a functional role for the glucocorticoidinduced TNF receptor. Immunity, 2002. 16(2): p. 311-23. 
392. Ermann, J., et al., Only the CD62L+ subpopulation of CD4+CD25+ regulatory $T$ cells protects from lethal acute GVHD. Blood, 2005. 105(5): p. 2220-6.

393. Iellem, A., et al., Unique chemotactic response profile and specific expression of chemokine receptors CCR4 and CCR8 by CD4(+)CD25(+) regulatory $T$ cells. The Journal of experimental medicine, 2001. 194(6): $\mathrm{p}$. 847-53.

394. Poggi, A. and M.R. Zocchi, Role of bone marrow stromal cells in the generation of human CD8+ regulatory $T$ cells. Human Immunology, 2008. 69(11): p. 755-9.

395. Trzonkowski, P., et al., Homeostatic repopulation by CD28-CD8+T cells in alemtuzumab-depleted kidney transplant recipients treated with reduced immunosuppression. American journal of transplantation : official journal of the American Society of Transplantation and the American Society of Transplant Surgeons, 2008. 8(2): p. 338-47.

396. Chen, H., et al., Impaired signaling in alloantigen-specific CD8+ $T$ cells tolerized in vivo: employing a model of Ld-specific TCR transgenic mice transplanted with allogenic hearts under the cover of a short-term rapamycin treatment. J Immunol, 1996. 157(10): p. 4297-308.

397. Yuling, H., et al., Essential role of sphingosine-1-phosphate receptor 1bearing $C D 8+C D 44+C C R 7+T$ cells in acute skin allograft rejection. Am J Transplant, 2008. 8(7): p. 1401-12.

398. Dault, L.A., C.S. Nagy, and J.A. Collins, Reversing cardiac transplant rejection with Orthoclone OKT3. Am J Nurs, 1989. 89(7): p. 953-5.

399. Hammond, E.H., et al., Relationship of OKT3 sensitization and vascular rejection in cardiac transplant patients receiving OKT3 rejection prophylaxis. Transplantation, 1990. 50(5): p. 776-82.

400. Sumrani, N., et al., OKT3 in severe early rejection: predictors for reversal in renal transplant recipients. Transplant Proc, 1990. 22(4): p. 1750-2.

401. Sutherland, F., et al., OKT3 resistant rejection in liver transplant patients. Transplant Proc, 1991. 23(1 Pt 2): p. 1413-4.

402. el-Ghoroury, M., et al., Comparison between methylprednisolone and OKT3 treatment for the first acute rejection episode in combined kidney and pancreas transplant recipients. Transplant Proc, 1995. 27(6): p. 31334.

403. Ochando, J.C., et al., Lymph node occupancy is required for the peripheral development of alloantigen-specific Foxp3+ regulatory T cells. J Immunol, 2005. 174(11): p. 6993-7005.

404. Koch, S.D., et al., Alloantigen-induced regulatory CD8+CD103+T cells. Hum Immunol, 2008. 69(11): p. 737-44.

405. Zhou, J., et al., Oral exposure to alloantigen generates intragraft CD8+ regulatory cells. J Immunol, 2001. 167(1): p. 107-13.

406. Prevosto, C., et al., Generation of CD4+ or CD8+ regulatory T cells upon mesenchymal stem cell-lymphocyte interaction. Haematologica, 2007. 92(7): p. 881-8. 
407. Zhang, L., et al., Regulatory $T$ cell (Treg) subsets return in patients with refractory lupus following stem cell transplantation, and TGF-betaproducing CD8+ Treg cells are associated with immunological remission of lupus. J Immunol, 2009. 183(10): p. 6346-58.

408. Godfrey, W.R., et al., In vitro-expanded human CD4(+)CD25(+) Tregulatory cells can markedly inhibit allogeneic dendritic cell-stimulated MLR cultures. Blood, 2004. 104(2): p. 453-61.

409. Villar, L.M., et al., Intrathecal synthesis of oligoclonal IgM against myelin lipids predicts an aggressive disease course in MS. J Clin Invest, 2005. 115(1): p. 187-94. 


\section{VITAE}

